\author{
UNIVERSITY OF SÃO PAULO \\ ENGINEERING SCHOOL OF SÃO CARLOS \\ DEPARTMENT OF STRUCTURAL ENGINEERING
}

PABLO AUGUSTO KRAHL

Lateral stability of ultra-high performance fiber-reinforced concrete beams with emphasis in transitory phases

Instabilidade lateral de vigas de concreto de ultra-alto desempenho reforçado com fibras com ênfase em fases transitórias 

PABLO AUGUSTO KRAHL

\title{
Lateral stability of ultra-high performance fiber-reinforced concrete beams with emphasis in transitory phases
}

\author{
VERSÃO CORRIGIDA \\ A versão original encontra-se na Escola de Engenharia de São Carlos
}

Text presented to the São Carlos School of Engineering of the University of São Paulo as one of the requisites for obtaining the degree Doctor in Civil Engineering (Structures).

Advisor: Ricardo Carrazedo

Co-advisor: Mounir Khalil El Debs

São Carlos 
AUTORIZO A REPRODUÇÃO TOTAL OU PARCIAL DESTE TRABALHO, POR QUALQUER MEIO CONVENCIONAL OU ELETRÓNICO, PARA FINS DE ESTUDO E PESQUISA, DESDE QUE CITADA A FONTE.

Ficha catalográfica elaborada pela Biblioteca Prof. Dr. Sérgio Rodrigues Fontes da EESC/USP com os dados inseridos pelo(a) autor(a).

Krahl, Pablo Augusto

Lateral stability of ultra-high performance fiber-reinforced concrete beams with emphasis in transitory phases / Pablo Augusto Krahl; orientador Ricardo Carrazedo; coorientador Mounir Khalil El Debs. São Carlos, 2018.

Tese (Doutorado) - Programa de Pós-Graduação em Engenharia Civil (Engenharia de Estruturas) e Área de Concentração em Estruturas -- Escola de Engenharia de São Carlos da Universidade de São Paulo, 2018.

1. Ultra-High Performance Fiber-Reinforced Concrete. 2. UHPC. 3. Lateral instability of beams. 4. Damage evolution laws. 5. Stress-strain relations. I. Título.

Eduardo Graziosi Silva - CRB - 8/8907 


\section{FOLHA DE JULGAMENTO}

Candidato: Engenheiro PABLO AUGUSTO KRAHL.

Título da tese: "Instabilidade lateral de vigas de concreto de ultra-alto desempenho reforçado com fibras com ênfase em fases fransitórias".

Data da defesa: 04/07/2018

Comissão Julgadora:

Resullado:

Prof. Titular Mounir Khalil El Debs

ARROYADO

(Orientador)

(Escola de Engenharia de São Carlos/EESC)

Prof. Associado Vladimir Guilherme Haach

APROVADO

(Escola de Engenharia de São Carlos/EESC)

Prof. Associado Antonio Domingues de Figueiredo

[Escola Politécnica/EP-USP)

Profa. Dra. Marla Cristina Vidigal de Lima

APROVADO

(Universidade Federal de Uberlândia/UFU)

Prof. Dr. Gustavo Henrique Siqueira

[Universidade Estadual de Campinas/Unicamp)

APROVADO

Coordenador do Programa de Pós-Graduaçāo em Engenharia Civil (Engenharia de Estruturas):

Prof. Titular Humberto Breves Coda

Presidente da Comissão de Pós-Graduação:

Prof. Associado Luís Fernando Costa Alberto 



\section{ABSTRACT}

KRAHL, P. A. Lateral stability of ultra-high performance fiber-reinforced concrete beams with emphasis in transitory phases. 2018. 191p. Thesis (Ph.D.) - School of Engineering of São Carlos, University of São Paulo, São Carlos, 2018.

The development of advanced fiber reinforced cement-based materials to provide higher strength, ductility, and durability, as ultra-high performance fiber-reinforced concrete (UHPFRC), enables the design of precast beams with thin sections and reduced self-weight to meet the required flexural performance. However, such slender elements when subjected to transitory phases, and possibly also in permanent stages, are prone to instability failure. So, the present study aims to provide experimental data and analytical solution for UHPFRC beams during the lifting phase, and studies about the other stages. This type of test is rare and was not reported for UHPFRC beams. For testing, the beams were lifted by inclined cables and subjected to a transversal load applied at midspan to induce lateral instability. The displacements of the beams were monitored with total station equipment. Also, a new analytical solution was proposed to predict the failure load of lifted beams and closed-form analytical solutions to predict the rollover load of beams supported by bearing pads and subjected to different loading conditions. Furthermore, there are limited data that characterizes the constitutive behavior of this material. In this context, the present research also focused on providing such laboratory results for UHPFRC with different fiber contents. Besides, analytical models for damage evolution and stress-strain relationship are proposed and applied in numerical simulations. From the results, the UHPFRC beams failed by instability with a load capacity 3.7 times smaller than the flexural load capacity. Furthermore, the analytical solution for lifting predicted the peak load of the experiment with great accuracy. Also, the proposed equations for beams on bearing pads accurately predicted the experimental results available in the literature. The analytical and experimental rollover loads differed by $4.37 \%$ and $13.6 \%$ for the two studied cases. From material, the stiffness degradation occurred rapidly in UHPFRC under tensile loading while occurred gradually in compression. Also, fiber content influenced toughness and degradation evolution significantly over the loading cycles. Proposed equations were utilized in the PlasticDamage model of Abaqus that predicted accurately damage growth and cyclic envelopes during all the phases of the tension, compression, and bending tests. The calibrated numerical model also predicted the experimental results with the UHPFRC beams. 
Keywords: Ultra-High Performance Fiber-Reinforced Concrete. UHPC. Lateral instability of beams. Damage evolution laws. Stress-strain relations. 


\section{RESUMO}

\section{KRAHL, P. A. Instabilidade lateral de vigas de concreto de ultra-alto desempenho}

reforçado com fibras com ênfase em fases transitórias. 2018. 191p. Tese (Doutorado) Escola de Engenharia de São Carlos, Universidade de São Paulo, São Carlos, 2018.

O desenvolvimento de materiais avançados à base de cimento reforçado com fibra para fornecer maior resistência, ductilidade e durabilidade, como o concreto de ultra-alto desempenho reforçado com fibras (UHPFRC), permite o projeto de vigas pré-moldadas com seções esbeltas e peso próprio reduzido que atendem desempenho estrutural requerido. No entanto, esses elementos delgados quando submetidos a fases transitórias e também em serviço são propensos a falhar por instabilidade. Então, o presente estudo tem por objetivo apresentar resultados experimentais e soluções analíticas para vigas de UHPFRC durante a fase de içamento e estudos sobre as outras fases. Este tipo de teste é raro e não foi reportado para vigas de UHPFRC. Para o teste, as vigas foram levantadas por cabos inclinados e submetidas a uma carga concentrada transversal aplicada no meio do vão para induzir a instabilidade lateral. Os deslocamentos das vigas foram monitorados com estação total. Além disso, uma nova solução analítica foi proposta para prever a carga de instabilidade das vigas içadas e soluções analíticas para prever a carga de tombamento de vigas suportadas por aparelho de apoio e submetidas a diferentes condições de carregamento. Além disso, existem poucos resultados experimentais que caracterizam o comportamento constitutivo deste material. Neste contexto, a presente pesquisa também se concentrou em fornecer tais resultados experimentais para UHPFRC com diferentes teores de fibras. Além disso, modelos analíticos para evolução de dano e relação tensão-deformação são propostos e aplicados em simulações numéricas. A partir dos resultados, as vigas em içamento falharam por instabilidade com uma capacidade de carga 3,7 vezes menor que a capacidade à flexão. Além disso, a solução analítica para içamento previu carga máxima do experimento com grande precisão. As equações propostas para vigas sobre aparelhos de apoio previram com precisão os resultados experimentais disponíveis na literatura. As cargas de tombamento analíticas e experimental diferiram em 4,37\% e 13,6\% para os dois casos estudados. Dos resultados do material, a degradação da rigidez ocorreu de maneira rápida no UHPFRC submetido à tração enquanto ocorreu gradualmente na compressão. $\mathrm{O}$ teor de fibras influenciou significativamente a tenacidade e a degradação nos ciclos de carregamento. As equações propostas foram utilizadas em um modelo de Dano acoplado à plasticidade que 
previu com precisão a evolução do dano e as envoltórias cíclicas durante todas as fases dos testes de tração, compressão e flexão. O modelo numérico calibrado também previu os resultados experimentais das vigas de UHPFRC.

Palavras-chave: Concreto de ultra alto desempenho. CUAD. Instabilidade lateral de vigas. Leis de evolução de dano. Relação tensão-deformação. 


\section{LIST OF PUBLICATIONS}

This thesis is based on the work contained in the following papers:

I - KRAHL, P. A.; CARRAZEDO, R.; EL DEBS, M. K. Lateral buckling analysis of precast UHPFRC beams. 4th International Symposium on Ultra-High Performance Concrete and High Performance Construction Materials. Anais... . v. 1, p.1-9, 2016.

II - KRAHL, P. A.; CARRAZEDO, R.; EL DEBS, K. M. Rollover stability of precast concrete beams supported by elastomeric bearing pads. Ibracon structures and materials journal, v. 10, n. 6, p. $1192-1204,2017$.

III - KRAHL, P. A.; CARRAZEDO, R.; EL DEBS, K. Mechanical damage evolution in UHPFRC: Experimental and numerical investigation. Engineering Structures, v. 170, p. 63-77, 2018.

IV - KRAHL, P. A.; CARRAZEDO, R.; EL DEBS, M. K. Analytical solutions for rollover instability of concrete beams on elastomeric bearing pads under different loading conditions. Engineering Structures, v. 174, p. 154-164, $2018 .$.

V - KRAHL, P. A.; MARTINS, D. DE O.; CARRAZEDO, R.; SILVA, I. DA; EL DEBS, M. K. Experimental and analytical studies on the lateral instability of UHPFRC beams lifted by cables. Composite Structures, v. Submitted, 2018. 



\section{SUMMARY}

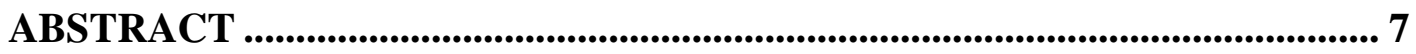

RESUMO

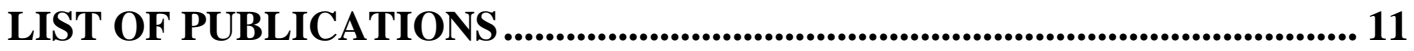

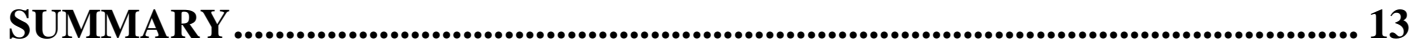

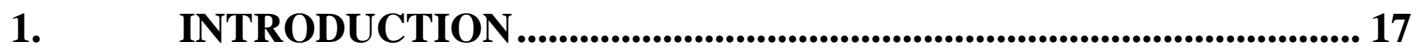

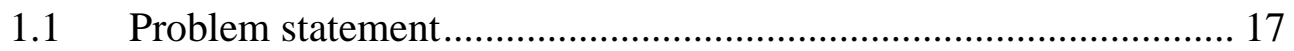

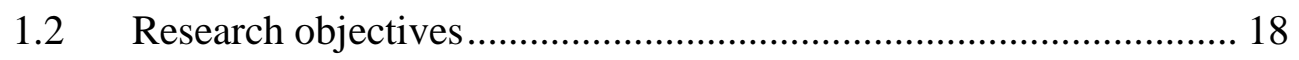

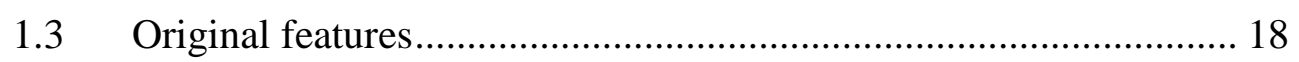

2. ULTRA-HIGH PERFORMANCE FIBER-REINFORCED

CONCRETE (UHPFRC) ..................................................................................................... 21

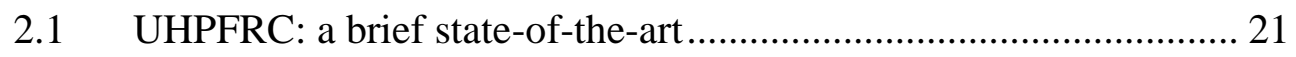

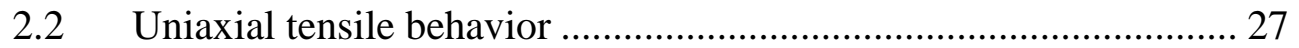

2.3 Uniaxial compressive behavior ................................................. 32

$2.4 \quad$ Biaxial and triaxial behaviors ........................................................ 39

2.5 Concrete Damage Plasticity constitutive model for UHPFRC ........ 42

2.6 Calibration of the CDP model...................................................... 44

3. STATE-OF-THE-ART OF LATERAL STABILITY OF

CONCRETE BEAMS IN TRANSITORY PHASES ................................................... 47

3.1 Review of design guidelines ................................................... 51

3.2 Reported experimental studies ............................................... 52

3.3 Critical analysis of available analytical solutions ......................... 63

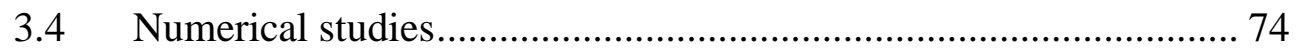

4. EXPERIMENTAL STUDY: MATERIALS AND METHODS ........ 85

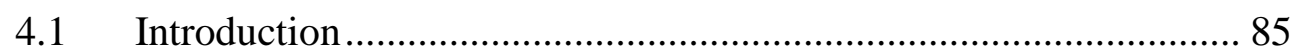

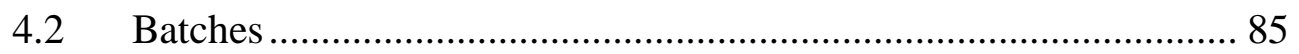

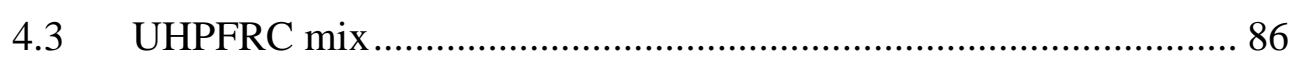

4.4 Mixing, sample preparation, and curing process ........................... 91

4.5 Uniaxial compressive test of UHPFRC ….................................... 92

4.6 Uniaxial tensile test of UHPFRC ................................................ 94 
4.7 Four point bending test of UHPFRC …..................................... 95

4.8 Loading cycles and experimental damage measurement ................ 95

4.9 Damage quantification through cyclic tests .................................... 96

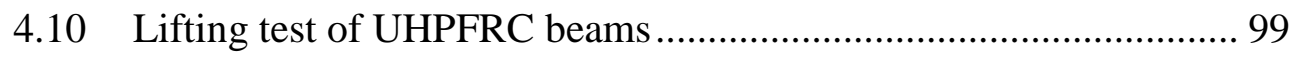

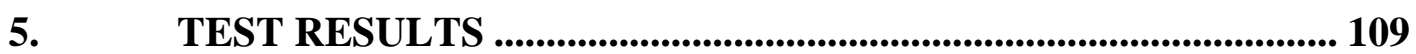

5.1 Monotonic tensile behavior...................................................... 109

5.2 Monotonic compressive behavior .............................................. 110

5.3 Monotonic flexural response ................................................... 120

5.4 Cyclic behavior and damage evolution in tension ....................... 125

5.5 Cyclic behavior and damage evolution in compression ............... 128

5.6 Cyclic behavior under flexure..................................................... 130

5.7 Lateral stability of UHPFRC beams lifted by cables .................... 131

6. ANALYTICAL PROPOSALS .................................................... 138

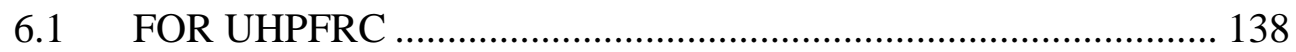

6.1.1 Compressive stress-strain behavior ............................................. 138

6.1.2 Tensile stress-strain behavior...................................................... 139

6.1.3 Damage evolution in compression ............................................ 141

6.1.4 Damage evolution in tension ................................................... 141

6.2 FOR LATERAL INSTABILITY OF BEAMS ........................... 142

6.2.1 Rayleigh-Ritz method .......................................................... 142

6.2.2 Instability of beams lifted by cables ......................................... 142

6.2.3 Instability of beams supported by bearing pads .......................... 147

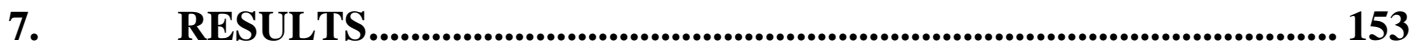

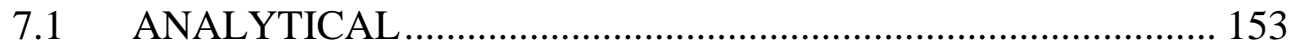

7.1.1 UHPFRC beams lifted by cables .............................................. 153

7.1.2 Concrete beams supported by bearing pads ................................ 154

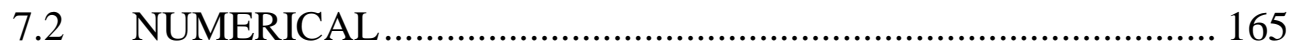

7.2.1 UHPFRC under uniaxial tensile and compressive cyclic loading 165

7.2.2 UHPFRC under flexural cyclic loading ...................................... 166

7.2.3 Numerical modeling of UHPFRC beam lifted by cables............... 169

7.2.4 Tilt behavior of concrete beam tested by Lima............................ 172

7.2.5 Tilt behavior of UHPFRC beam .............................................. 176 


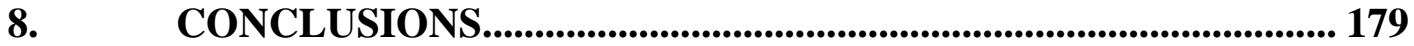

9. PROSPECTS FOR FUTURE RESEARCH ................................ 183

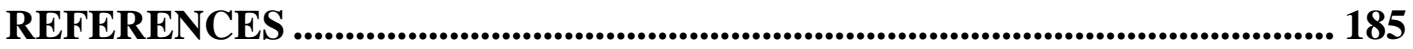




\section{INTRODUCTION}

\subsection{Problem statement}

The development of advanced fiber reinforced cement-based materials to provide higher strength, ductility and durability enable the design of structural elements with slender sections and reduced self-weight. Then, the current structural elements can be optimized.

Ultra-high performance fiber reinforced concrete (UHPFRC) is a particular class of such materials that have been increasingly researched. This cement-based composite is typically combined with surface treated straight steel fibers. Bridge girders and roof beams are promising structural elements to be designed with UHPFRC. In some projects these elements can be required to reach long spans, then UHPFRC can provide satisfactory structural behavior and possible low self-weight obtained by performing section optimization. In the present research, the term UHPFRC will be utilized when referring to the composite with fiber addition and UHPC to mention the material without fibers.

Also, it is known that precast elements are subjected to transitory phases, like lifting, transport and when only supported only by bearing pads, (EL DEBS, 2017). In these situations arise the concern with instability problems due to the poor constraint against toppling provided by the supports and, also, the increased slenderness provided by UHPFRC. Thus, the development of new and optimized sections requires the verifications of the safety against the instability of the UHPFRC beams under transitory situations, besides the required performance in service and ultimate stages be guaranted. As there is the tendency of development of thin sections, at service can raise the concern with localized instability. Furthermore, it is worth emphasizing that the code recommendations, as fib Model Code (2010), are unsafe for lateral stability of normal strength concrete beams in transitory phases, (DE LA FUENTE ANTEQUERA; CAVALARO; GARCÍA, 2017), and do not exist for UHPFRC beams. This absence is mainly due to the lack of experimental researches in this field.

Although the technology to produce UHPFRC is available since the mid-1990s, the effective application of this material in civil structures is recent because of the development of competitive and eco-friendly admixtures that do not need special treatments. However, few codes provide design guidelines for fiber-reinforced materials with such high performance, two examples are the Japan and French Societies of Civil Engineers, 
respectively JSCE (2002) and AFGC (2013). Thus, the constitutive behavior of such materials is under continuous research due to numerous existing gaps.

From the previous arguments, the present research focused on two main topics that are the instability problem of UHPFRC beams in transitory phases of construction and experimental characterization of the constitutive behavior of the developed UHPFRC.

\subsection{Research objectives}

The main objective of the present thesis is the study of the lateral stability of slender UHPFRC beams. The following specific objectives were further established for this research:

- Experimental study of the constitutive behavior of UHPFRC under monotonic and cyclic loading. Then, propose analytical solutions for the stress-strain relationship and damage evolution, for both tensile and compressive stress cases;

- Calibration of a constitutive Plastic-Damage model to represent the nonlinear behavior of UHPFRC;

- Study of lateral stability of slender UHPFRC beams lifted by cables through experimental and numerical analysis;

- Propose analytical solutions for concrete beams lifted by cables and supported by bearing pads.

\subsection{Original features}

The research presented in this thesis had the first focus on material behavior (UHPFRC), and then the instability problems of UHPFRC beams lifted by cables. From this research the following results are novel in the correspondent fields:

- Damage evolution equations, derived from the experimental program developed for ultra-high performance fiber-reinforced concrete (UHPFRC) subjected to uniaxial tensile and compressive loads. These equations are of great significance for numerical modeling of the degradation mechanisms of this emerging cementbased material;

- The calibration of widely used failure criteria for ordinary concrete was made for UHPFRC. This criterion is part of a plastic-damage constitutive model. 
Moreover, the damage evolution in the model is represented by the damage evolution equations developed in the present research;

- New analytical solutions to predict rollover load of unbraced precast beams supported by elastomeric bearing pads. Furthermore, a focus was given to analytical equations for different loading conditions from the typical construction load (self-weight). This consideration is because, in the published experimental studies, other concentrated loads are applied in addition to the self-weight;

- Experimental test results of lateral instability of ultra-high performance fiberreinforced concrete (UHPFRC) beams supported by lifting cables. The results are of great significance to the field of thin elements made of UHPFRC that are in current development. Besides, new analytical solution was proposed to predict the experimental behavior of such beams. 


\section{ULTRA-HIGH PERFORMANCE}

FIBER-REINFORCED

\section{CONCRETE (UHPFRC)}

\subsection{UHPFRC: a brief state-of-the-art}

According to (NAAMAN; WILLE, 2012), Fiber-Reinforced Concrete (FRC) had experienced milestones in the composite progress to achieve what is today called Ultra-High Performance Fiber-Reinforced Concrete (UHPFRC). Besides the matrix and fiber progress, the understanding of its interaction made possible the development of a composite with high strength, toughness, energy absorption, stiffness, durability, corrosion resistance and fire resistance. Table 1 presents the summary of chronological advances to achieve UHPFRC.

Table 1 - Chronological advances in the matrix and fibers since the 1960's, (NAAMAN; WILLE,

\begin{tabular}{|c|c|c|}
\hline Decade & Cementitious Matrix and Concrete & Fiber \\
\hline 1970 's & $\begin{array}{l}\text { - Better understanding of hydration reactions; gel } \\
\text { structure; } \\
\text { - Better understanding shrinkage, creep, porosity, ... } \\
\text { - High strength concrete to } 50 \mathrm{MPa} \text { in practice } \\
\text { - Development of water reducers } \\
\text { - Advances in concrete treatments and curing conditions }\end{array}$ & $\begin{array}{l}\text { - Smooth steel fibers; } \\
\text { normal strength } \\
\text { - Glass fibers } \\
\text { - Some synthetic fibers }\end{array}$ \\
\hline 1980 's & $\begin{array}{l}\text { - Increased development of chemical additives: HWRA, } \\
\text { etc... } \\
\text { - Increased utilization of fly ash and silica fume, and other } \\
\text { mineral additives, etc... } \\
\text { - Increased flowability (flowable concrete) } \\
\text { - Reduction in W/C ratio; } \\
\text { - High-Strength-Concrete terminology: up to } 60 \mathrm{MPa} \text {; } \\
\text { special high strength: up to } 80 \mathrm{MPa} \text {; exotic high strength } \\
\text { (special aggregate and curing): up to } 120 \mathrm{MPa} \\
\text { - High-Performance-Concrete terminology: high-strength- } \\
\text { concrete with improved durability properties. }\end{array}$ & $\begin{array}{l}\text { - Deformed steel fibers: } \\
\text { normal and high strength } \\
\text { - Low-modulus synthetic } \\
\text { fibers (PP, nylon, etc..) } \\
\text { - Increased use of glass } \\
\text { fibers } \\
\text { - Micro fibers } \\
\text { - High performance } \\
\text { polymer fibers (carbon, } \\
\text { Spectra, Kevlar, etc..) }\end{array}$ \\
\hline 1990 's & $\begin{array}{l}\text { - Increased development in chemical additives: } \\
\text { superplasticizers; viscosity agents; etc.... } \\
\text { - Increased use of supplementary cementitious materials } \\
\text { as cement replacement } \\
\text { - UHPC: application of concept of high packing density; } \\
\text { addition of fine particles; low porosity; lower water to } \\
\text { cementitious ratio; } \\
\text { - Self consolidating concrete; self compacting concrete; }\end{array}$ & $\begin{array}{l}\text { - New steel fibers with a } \\
\text { twist (untwist during pull- } \\
\text { out) } \\
\text { - PVA fibers with chemical } \\
\text { bond to concrete } \\
\text { - Improved availability of } \\
\text { synthetic fibers }\end{array}$ \\
\hline 2000's & $\begin{array}{l}\text { - Increased developments of proprietary and non- } \\
\text { proprietary UHPC/UHP-FRC } \\
\text { - UHPC: improved understanding of high packing density; } \\
\text { application of nanotechnology concepts }\end{array}$ & $\begin{array}{l}\text { - Ultra high strength steel } \\
\text { fibers: smooth or } \\
\text { deformed with diameters } \\
\text { as low as } 0.12 \mathrm{~mm} \text { and } \\
\text { strengths up to } 3400 \\
\mathrm{MPa} \\
\text { - Carbon nano-tubes; } \\
\text { carbon nano-fibers }\end{array}$ \\
\hline 2020 's & $\begin{array}{l}\text { - Increased understanding of the cementitious matrix at } \\
\text { the nano-scale } \\
\text { - ..???... }\end{array}$ & $\begin{array}{l}\text { - Carbon nano-fibers, } \\
\text { graphene, ... } \\
\text { - } \quad \ldots ? ? ? \text { ?... }\end{array}$ \\
\hline
\end{tabular}


UHPFRC is commonly defined by its compressive strength. For example, UHPFRC is a cement-based composite with compressive strength superior to $150 \mathrm{MPa}$. However, there is currently no consensus on this definition. Several papers were published calling UHPFRC as a cement-based composite with compressive strength even below $150 \mathrm{MPa}$. Then, seems to be a more sensible view divide UHPFRC into classes relative to its performance. (TOUTLEMONDE; DELORT, 2016) presented the categories defined by the new French Code NF P 18-470, Table 2.

Table 2 - Compressive strength classes for UHPFRC and associated limiting values,

(TOUTLEMONDE; DELORT, 2016)

\begin{tabular}{ccc}
\hline Compressive strength class & \multicolumn{2}{c}{ Limiting values } \\
\hline & $\begin{array}{c}\text { Minimum cylinder } \\
\text { characteristic strength } \\
\mathrm{f}_{\text {ck-cyl }}\end{array}$ & $\begin{array}{c}\text { Indicative minimum characteristic } \\
\text { strength on cubes } \\
\text { MPa }\end{array}$ \\
\hline UHPC 130/145 & 130 & MPa \\
$150 / 165$ & 150 & 145 \\
$175 / 190$ & 175 & 165 \\
$200 / 215$ & 200 & 190 \\
$225 / 240$ & 225 & 215 \\
$250 / 265$ & 250 & 240 \\
& & 265 \\
\hline
\end{tabular}

${ }^{1}$ Intermediate strength levels rounded to the nearest $5 \mathrm{MPa}$ may be used.

Furthermore, the French code classifies UHPFRC also due to its tensile behavior, workability, thermal treatment, improved potential durability, and abrasion. The tensile classification consists in comparing the first cracking strength $f_{c t}$ and the post-cracking strength $f_{c t f}$, which the stress level at which localization occurs. Average $\left(f_{c t m}\right.$ and $\left.f_{c t f m}\right)$ and characteristic $\left(f_{c t k}\right.$ and $\left.f_{c t f k}\right)$ values are considered for crack and peak strength. This classification divides UHPFRC according to the post-cracking regime, i.e., strain-softening, low strain-hardening or high strain-hardening. The designation is

$$
\begin{aligned}
& \left.\begin{array}{l}
\frac{f_{c t f m}}{1.25}<f_{c t m} \\
\frac{f_{c t f k}}{1.25}<f_{c t k}
\end{array}\right\} \text { Strain-softening } \\
& \left.\begin{array}{l}
\frac{f_{c t f m}}{1.25} \geq f_{c t m} \\
\frac{f_{c t f k}}{1.25}<f_{c t k}
\end{array}\right\} \text { Low strain-hardening }
\end{aligned}
$$


$\left.\begin{array}{l}\frac{f_{c t f m}}{1.25} \geq f_{c t m} \\ \frac{f_{c t f k}}{1.25} \geq f_{c t k}\end{array}\right\}$ High strain-hardening

Where 1.25 is a factor called $K_{\text {global }}$ that accounts for the placement effect of UHPFRC.

In the 1990's, the development of concretes with very high strength was expensive and required special technics for production. However, today the search for a cost-effective, self-compacting and sometimes eco-friendly UHPFRC has increased its potential and competitiveness when compared to the other most common structural solutions. (SERNA et al., 2015) developed a study concerning the comparison between the costs of UHPFRC solution against High-Performance Concrete (HPC-50) and Steel solutions of a pedestrian bridge with $42.81 \mathrm{~m}$ of the span. Table 3 presents the results of the study.

Table 3 - Comparison of costs for the solutions with UHPFRC, HPC-50, and Steel of the pedestrian bridge over the Ovejas Ravine (SERNA et al., 2015)

\begin{tabular}{lcccc}
\hline & & Steel & HPC-50 & UHPFRC \\
\hline Material Cost & $€ / \mathrm{m}^{3}$ & 7810 & 60 & 1500 \\
Total volume & $\mathrm{m}^{3}$ & 8,6 & 101,5 & 26,7 \\
Total weight & Ton & 67 & 253,7 & 66,8 \\
Structural cost & $€$ & 163.800 & 124.000 & 185.000 \\
Material cost & $\%$ & 40,9 & 4,9 & 21,6 \\
Fabrication cost & $\%$ & 59,1 & 95,1 & 78,4 \\
Reinforcements & $\mathrm{kg} / \mathrm{m}^{2}$ & & 77 & 57 \\
Equipment & $€$ & 35.000 & 32.000 & 17.000 \\
Transport and assembling & $€$ & 15.000 & 31.000 & 18.000 \\
Substructures & $€$ & 17.000 & 26.000 & 17.000 \\
Total cost & $€$ & 230.800 & 213.000 & 237.800 \\
\hline
\end{tabular}

Given only the total costs, the solution with UHPFRC was $3.0 \%$ and $11.6 \%$ higher than respectively the Steel and HPC-50 solutions. Figure 1 presents the pedestrian bridge constructed with UHPFRC. 


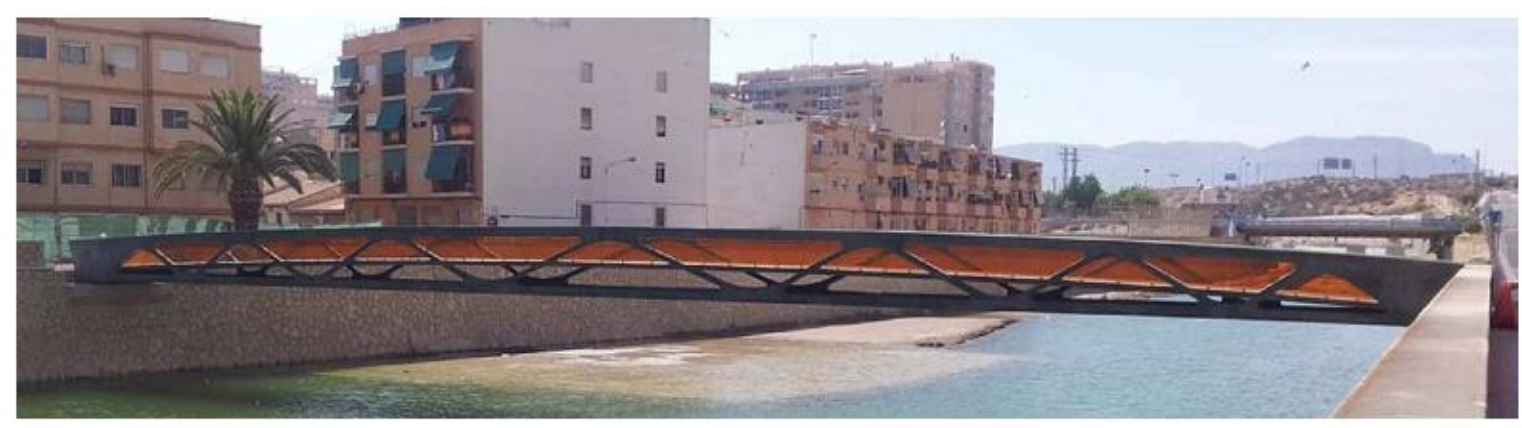

Figure 1 - UHPFRC pedestrian bridge over the Ovejas Ravine, Alicante

Due to the possible development of UHPFRC with local materials and nonspecial technics, several types of research have been conducted on many fields of structural engineering. Static and dynamic mechanical properties in material and structural scale were already investigated, as well as, durability, rehabilitation, and sustainability. Besides, several applications on real structures have been reported. These affirmations are confirmed by the number of review papers recently published, which are summarized in Table 4 . The subjects treated in the reviews are numbered below the table.

Table 4 - Summary of the main subjects treated in the reviews

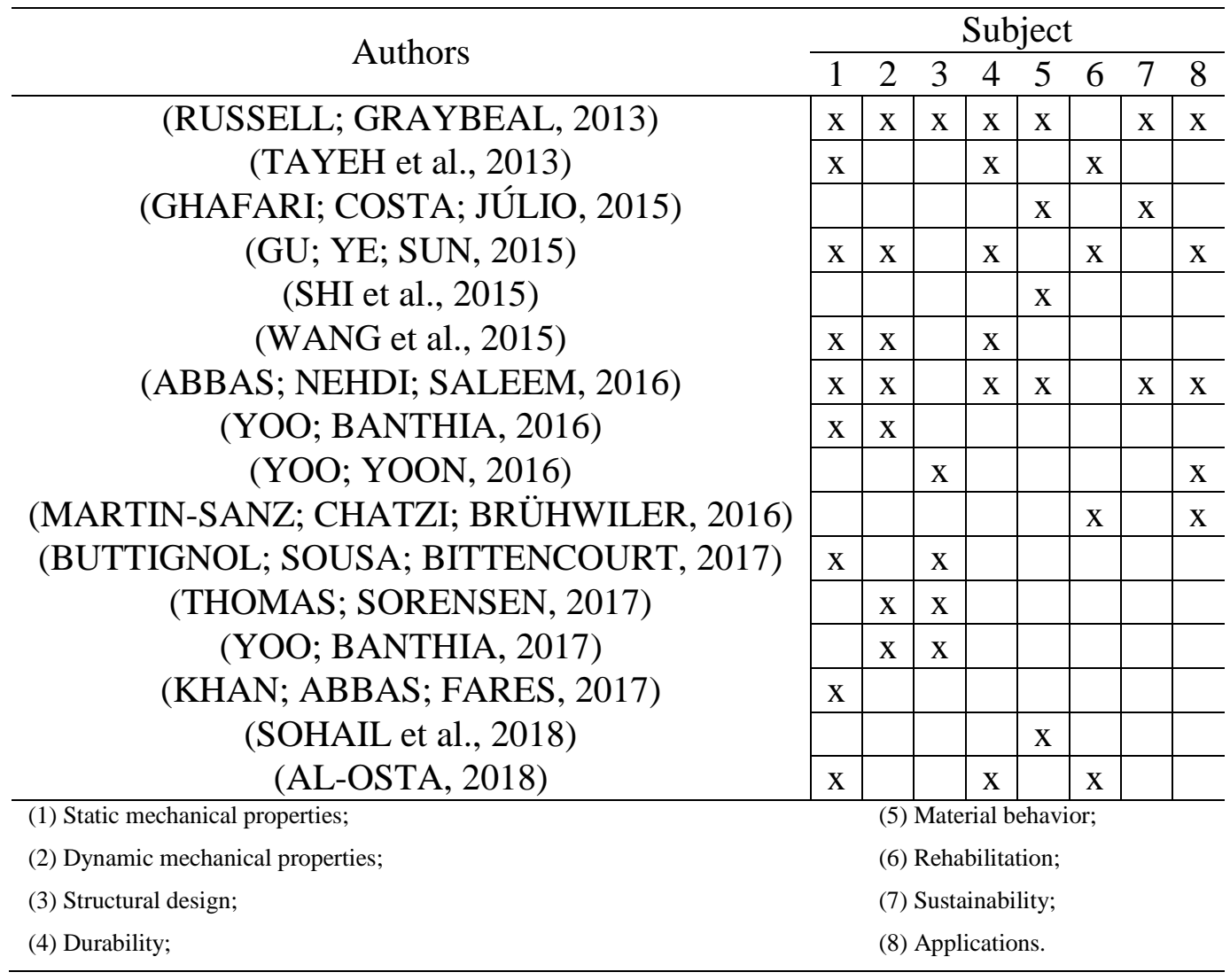

Some of the subjects addressed in the reviews are used in the present research, for example, static mechanical properties, structural design and material characteristics and 
behavior. It should be pointed out that no review reported investigations about instability problems in UHPFRC structural elements, which becomes a concern as is expected that the structural elements tend to be thin and lightweight due to the smaller consumption of material, allowed by UHPFRC, but reaching at the same time the required performance. Slender columns, beams, shell, arches, trusses are promising solutions when utilizing UHPFRC that, besides the high performance, brings an aesthetic appeal. Thus, the literature in this field is briefly presented here concerning instability problems. (ESCOBARCASTILLO et al., 2012) investigated the buckling capacity of thin-walled UHPFRC columns with I cross-section. The slender elements have a wall thickness of $25 \mathrm{~mm}$ and no steel rebars. Figure 2 presents the experimental load versus lateral deflection response of the test and the cross-section of the column.
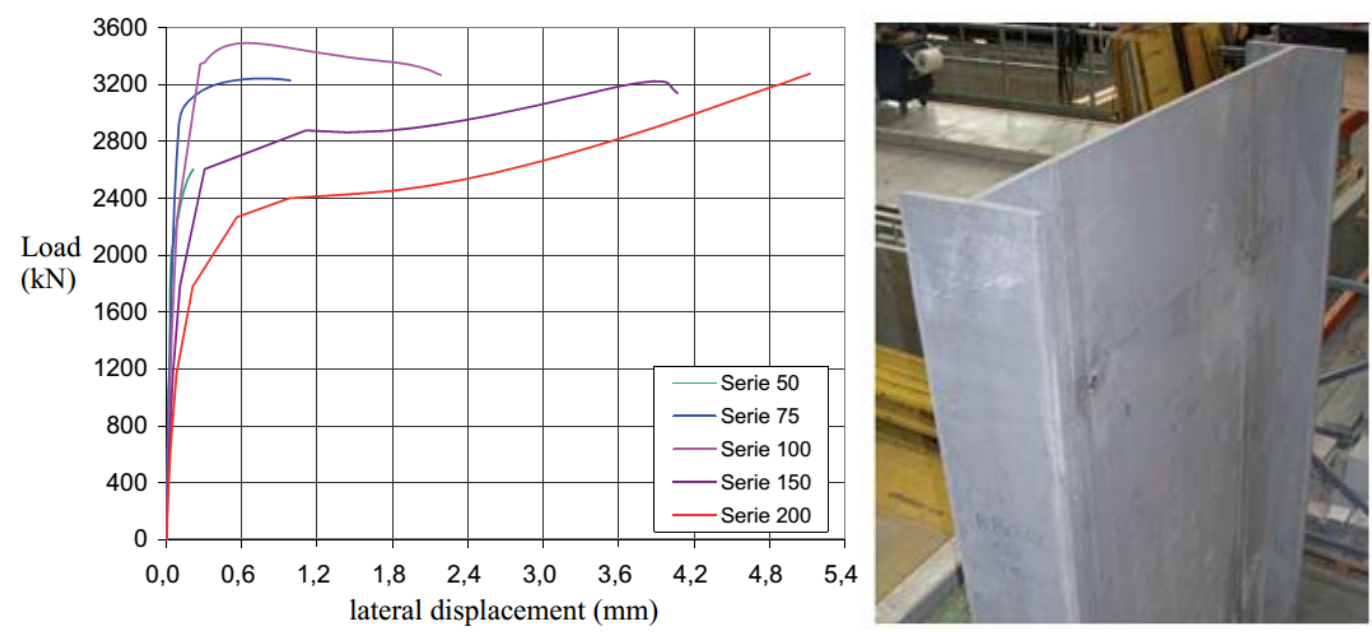

Figure 2 - Experimental results of (ESCOBAR-CASTILLO et al., 2012)

Buckling limited the strength, however, ductile behavior was observed after cracking for the series 75 to 200, as shown in Figure 2. The series categories correspond to the distance between the flanges of the specimens. (ILLICH; TUE; FREYTAG, 2014) studied the experimental behavior of slender prestressed UHPFRC columns. The structural elements presented a ductile behavior. The slenderness of such components could be increased for an acceptable buckling performance. 


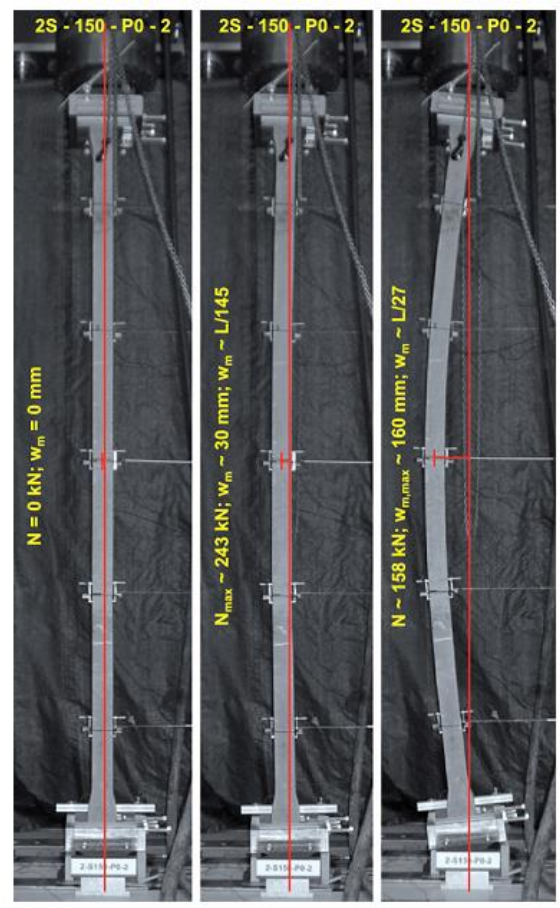

Figure 3 - Column tested by (ILLICH; TUE; FREYTAG, 2014)

(LECHNER; FISCHER, 2015) studied the stability of slender wall panels subjected axial load, as (ESCOBAR-CASTILLO et al., 2012). The tested panels did not have flanges, so the lateral stiffness was small. However, a ductile behavior was also observed after buckling. (SCHMIDT; CURBACH, 2016) studied the buckling capacity of UHPFRC columns without steel rebars and with rectangular and square cross-sections and, from this study, (SCHMIDT; CURBACH, 2017) investigated the shape optimization relative to buckling of such columns. They observed improvements in the instability behavior due to the shape modifications. These studies showed the potential for the development of slender elements with UHPFRC. So, the field is still open for research. For example, there is no reported experimental study on the stability performance of slender precast UHPFRC beams. For this reason, the main focus of the present research is investigating lateral buckling of beams in transitory phases. Besides, preliminary numerical and analytical studies were performed by (KRAHL; CARRAZEDO; EL DEBS, 2016).

Another research field that is under current development, and, also was not treated on the presented reviews is about the damage mechanisms and its evolution during mechanical loading in UHPFRC. Such characteristics are fundamental for the development of constitutive models to predict numerical and analytically the behavior of structural elements made of UHPFRC. The damage mechanisms have been studied through several technics as acoustic emission (KRAVCHUK; LANDIS, 2018), X-ray computed tomography 
(TRAINOR et al., 2013a) and Scanning electron microscopy (GU et al., 2018). Also, the quantification of the damage mechanisms has been done by cyclic tests, as (PASCHALIS; LAMPROPOULOS, 2016) for the tensile behavior of UHPFRC. The quantification of the damage mechanisms for UHPFRC was not reported before the current research, (KRAHL; CARRAZEDO; EL DEBS, 2018).

\subsection{Uniaxial tensile behavior}

In the design of conventional strength concrete structures, tensile strength is commonly neglected in the prediction of ultimate capacity. However, UHPFRC can carry tensile stresses after the first cracking even at failure load. Depending on the efficiency of the fiber/matrix interaction, UHPFRC can present strain-hardening behavior. Then, the material has the peak strength higher than the cracking resistance. Thus, the ductility and toughness can be considered in the design of UHPFRC structures.

As is known, cement-based materials have a system of distributed pores, air voids, and microcracks before carrying any load. The scale of this discontinuities depends on the particle size distribution of the materials that composed the matrix. Due to its optimized gradation and higher content of cement, UHPFRC has typically smaller pores and microcracks and a densified cementitious matrix at the interfacial transition zone (ITZ). The utilization of silica fume amplifies these enhancements in the mix and when possible heat curing after cast. The mentioned discontinuities can be found at the ITZ and in the bulk matrix. For UHPFRC, the weak interface is between matrix and fibers as shown in Figure 4.

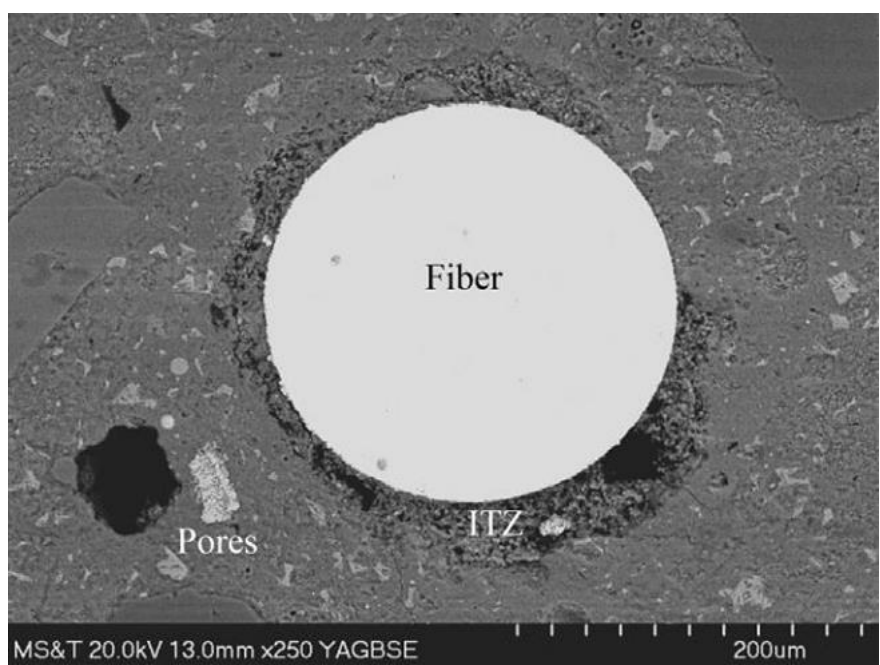

Figure 4 - Backscatter scanning electron microscopy of pores and interface between fiber and matrix, after (WU; SHI; KHAYAT, 2016) 
The early age flaws in cement-based matrixes are commonly attributed to segregation during casting, shrinkage and thermal cracking, (SHAH; SWARTZ; OUYANG, 1995). From there on, as stress is applied, these discontinuities starts to propagate and interconnect from ITZ and bulk matrix causing localization. The tensile macrocrack appearance is unstable and fast. When fibers are added to the matrix, the fracture processes are considerably influenced. Besides the increase in the heterogeneity of the composite, the fibers retain the velocity of macrocrack formation, in some cases forming multiple cracking that promotes ductility and toughness. Shortly before and after the first crack, fibers start to debond and develop frictional stresses at the interface with the matrix, Figure 5.

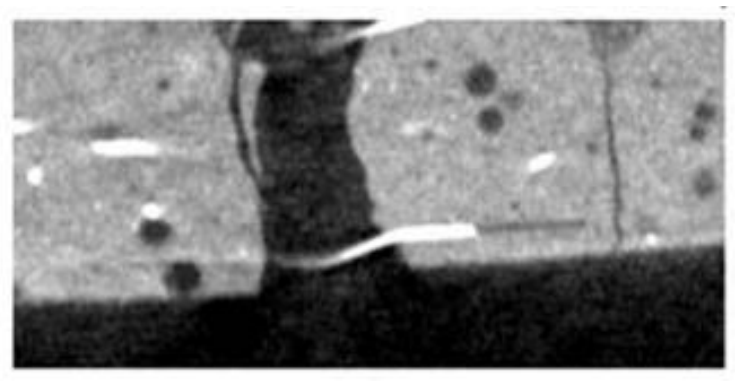

(a)

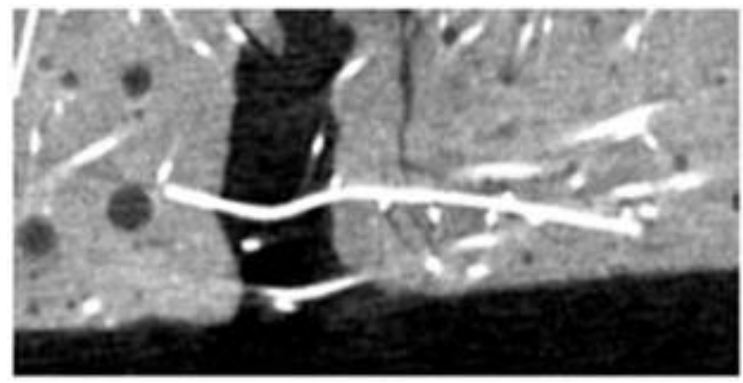

(b)

Figure 5 - Examples of degradation mechanisms developed between fiber and matrix from X-ray computed tomography, (FLANDERS; RUSHING; LANDIS, 2016)

Also, when the fiber is not normal to the crack faces, as in Figure 5, more energy is dissipated due to the fiber bending during the pullout. Figure 6 (a) and (b) present the results of pullout tests from a UHPFRC matrix with fibers aligned and inclined 15 degrees with the direction of load, respectively.

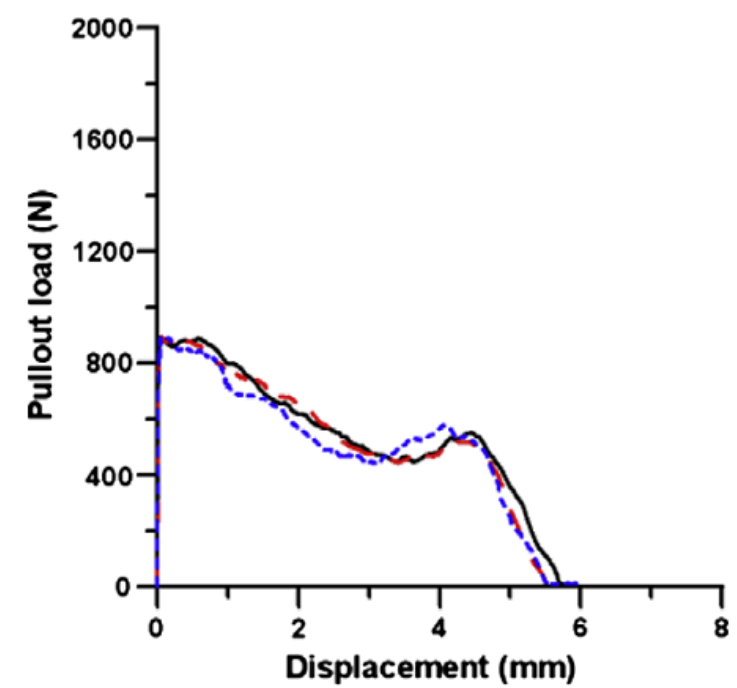

(a) fiber angle of $0^{\circ}$

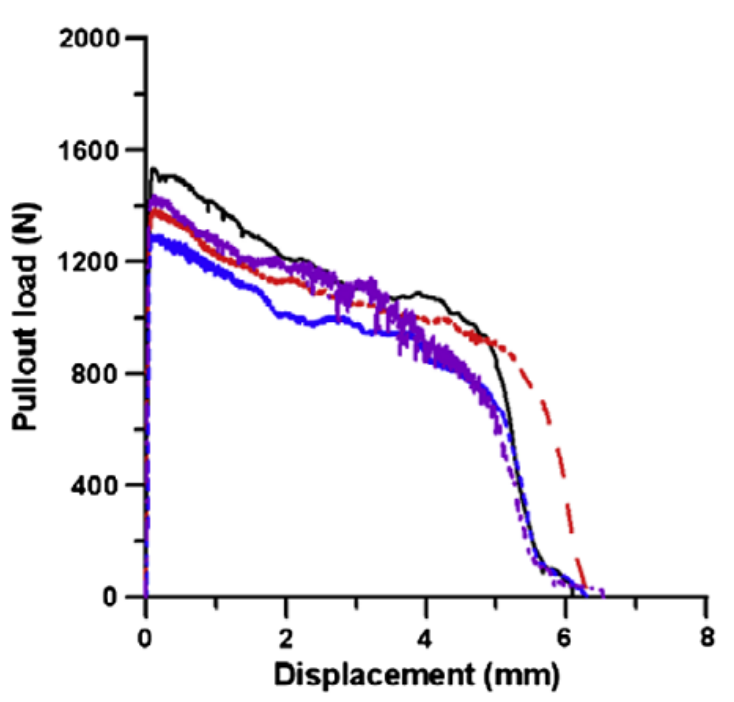

(b) fiber angle of $15^{\circ}$

Figure 6 - Fiber pullout with different inclinations, from (LEE; KANG; KIM, 2010) 
The same degradation mechanisms can occur at different nonlinear stages because the activation of the fibers in different regions of the structural element can be gradual due to the redistribution property provided by fibers. The utilization of fibers with mechanical anchorage can be restricted in cases that the matrix presents high strength, as UHPC. The fibers must have a compatible higher strength so as not fail, which is not desirable, see Figure 7.

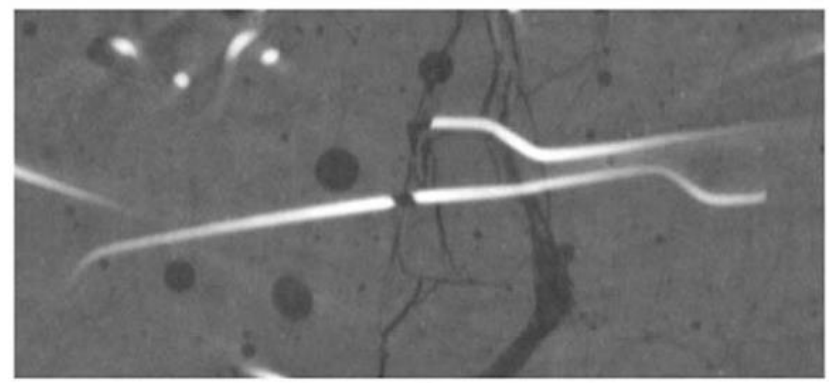

Figure 7 - Fiber rupture in a cracked region, (FLANDERS; RUSHING; LANDIS, 2016)

Depending on the fiber content and designed matrix, UHPFRC can present strainhardening or softening behavior. For strain-hardening case, multiple cracking occurs and the stress carrying capacity increases until the peak stress. Then, a macrocrack appears with a consequent drop in load, Figure 8 (a). In the softening behavior, only this second stage is registered because the composite has a low capacity of redistribution, Figure 8 (b). The redistribution occurs due to the efficiency in crack bridging by fibers that depends on factors as the surface roughness of the fibers and the matrix composition. During softening, the fiber slip at macrocracks prevails to the redistribution or stress transfer between crack faces. Thus, this crack tends to open, and fiber slip and pull-out are the main damage mechanisms.

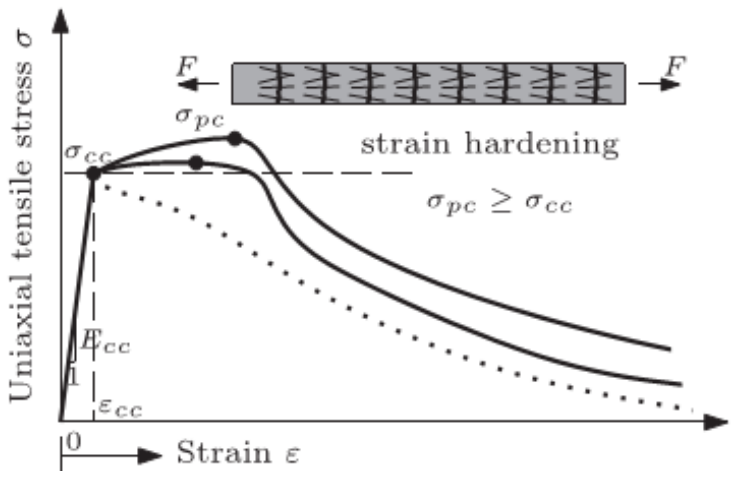

(a)

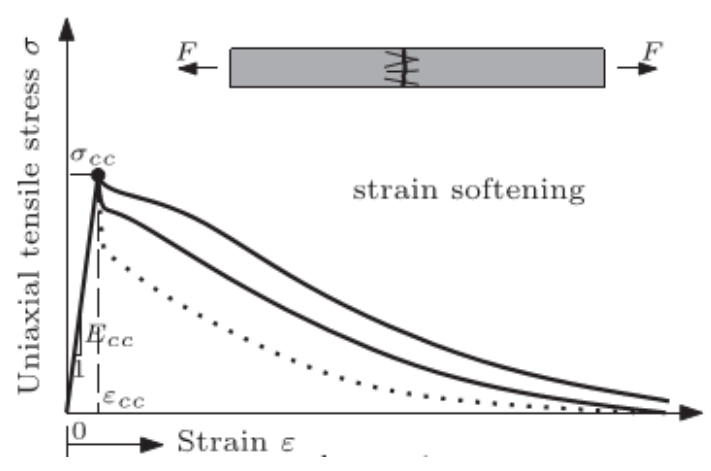

(b)

Figure 8 - (a) Strain-hardening and (b) softening behavior of UHPFRC [(WILLE; EL-TAWIL; NAAMAN, 2014)]

Thus, the fiber-matrix interaction performs a fundamental role in the response of UHPFRC. Fibers with high surface area combined with cementitious composites designed 
through packing optimization tend to have better adhesional and frictional bonding. Besides, the first mechanism can be improved by enhancing the properties of the interfacial transition zone with additions as silica fume, (WU; SHI; KHAYAT, 2016), and nano silica, (WU; KHAYAT; SHI, 2017). Figure 9 presents the pull-out response of straight steel fibers from UHSC with five different silica fume contents ( 0 - $25 \%$ by the mass of binder), (WU; SHI; KHAYAT, 2016). The best performance was for fractions of silica fume between $15-25$ \%. (WU; KHAYAT; SHI, 2017) investigated the influence of portions of nano silica between $0-2 \%$ of the cement weight on the pull-out behavior of UHSC. The content that presented the more significant improvement was $1 \%$ of nano silica, Figure 10.

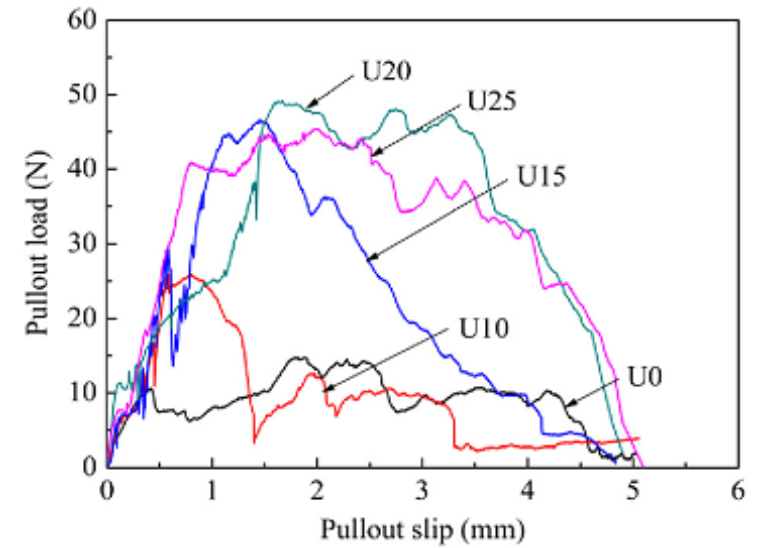

Figure 9 - Pull-out results for UHSC with different contents of silica fume, after (WU; SHI; KHAYAT, 2016)

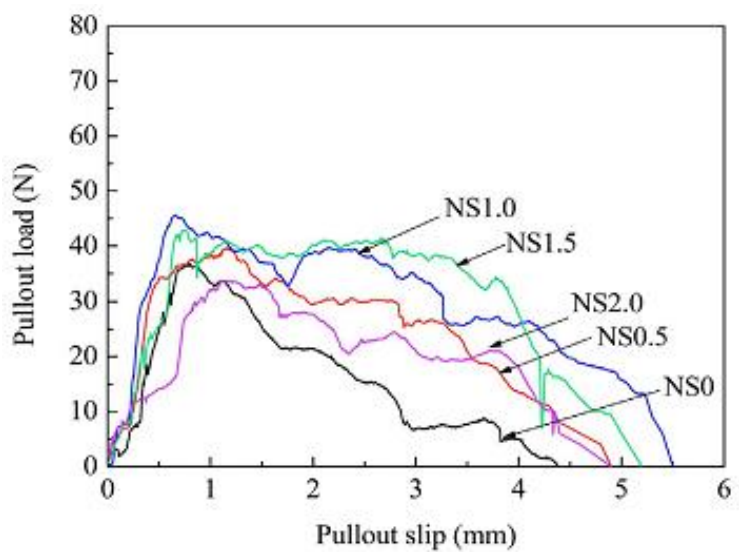

Figure 10 - Pull-out results for UHSC with varying volumes of nano silica, after (WU; KHAYAT; SHI, 2017)

Also, fiber surface coating commonly enhances fiber/matrix interaction due to the increase in roughness. The material deposited on fiber can be copper, brass, and other metals. Therefore, due to the enhanced adhesion and friction with the dense matrix, the application of smooth straight steel fibers to produce UHPFRC is commonly observed.

These material characteristics reflect the overall stress-strain behavior of UHPFRC under tensile loading. Then, when developing UHPFRC mixtures, strain hardening behavior under tension is desired, because the material can dissipate more energy and present higher durability, due to the occurrence of multiple microcracking.

\section{Analytical stress-strain behavior}

In literature, there are few models to predict the whole stages of the response of UHPFRC under tension. Most of the researchers consider the stage of localization with a different measure from the strain hardening branch. The first by crack opening and the second by strain. Thus, simulate strain hardening of UHPFRC becomes difficult, because, 
firstly the material presents "uniform" strain distribution between the beginning of multiple cracking stage and peak load, and then, crack-opening until rupture, as presented in Figure 8 (a). An approximative way to overcome this issue is to consider the stage of localization as an average uniform cracking formation. Through energy measurements, it is possible to consider all the degradation mechanisms for a specific volume of UHPFRC and determine an approximate stress-strain relation. This strategy enables to simulate UHPFRC with numerical models that do not account for discrete cracks and fibers.

(WILLE; EL-TAWIL; NAAMAN, 2014) developed a study concerning the tensile behavior of UHPFRC with three types of fibers, and each with three different volumes. They presented approximations for elastic, strain hardening and softening tensile parameters. The last two are energy-based parameters. The energy for the strain-hardening branch corresponds to the area under the stress-strain curve, and for the softening range is the area below the stress-crack opening curve. Equations (4) to (6) determine the matrix tensile strength $\sigma_{c c}(\mathrm{MPa})$, composite tensile strength $\sigma_{p c}(\mathrm{MPa})$, the energy dissipated until peak load $g_{f}\left(\mathrm{~kJ} / \mathrm{m}^{3}\right)$ and the energy dissipated after peak $G_{f}\left(\mathrm{~kJ} / \mathrm{m}^{2}\right)$. All variables are a function of the fiber content $V_{f}$ and presented in Figure 11.

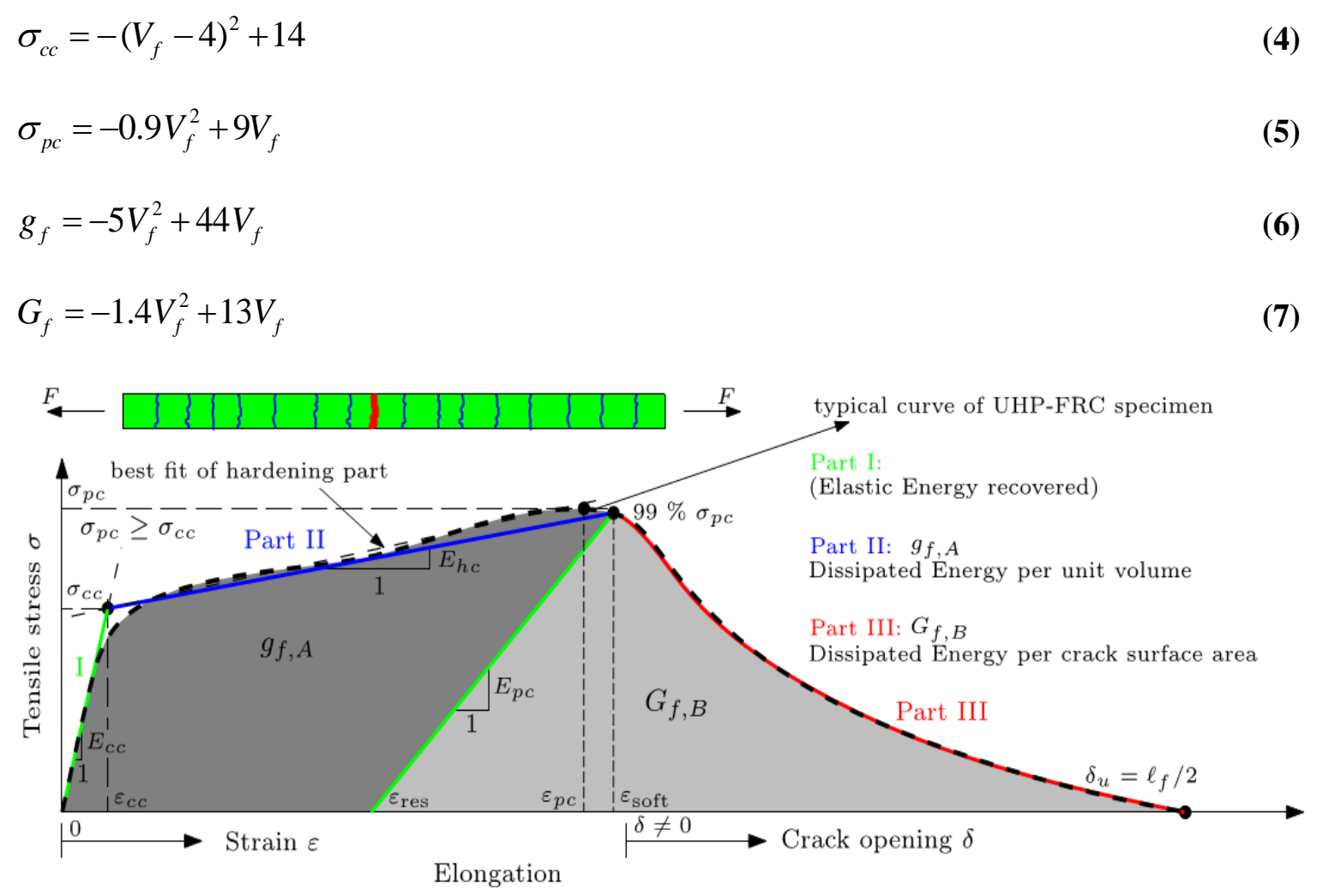

Figure 11 - Energy absorption prior to softening 


\subsection{Uniaxial compressive behavior}

The processes of cracking evolution until peak stress of plain concrete under uniaxial compression are well established, (HSU et al., 1963), and accepted, (SHAH; SWARTZ; OUYANG, 1995), (VAN MIER, 1997), (NEVILLE, 2012) and (MEHTA; MONTEIRO, 2013). Similar processes are expected in UHPFRC. However, the time of occurrence and scale are different. In general, the process consists of, firstly, bond cracks initially presented in the ITZ starts to propagate at stress levels between 30 and $70 \%$ of the peak stress. After, coalescence of pores and microcracks of the ITZ and the bulk matrix forms a continuous network of cracks at stress levels of 70 and $90 \%$. At this stage, concrete starts to dilate, and at peak, localization occurs. See in Figure 12 the stress-strain relationship until peak and the crack propagation stages presented by (HSU et al., 1963).

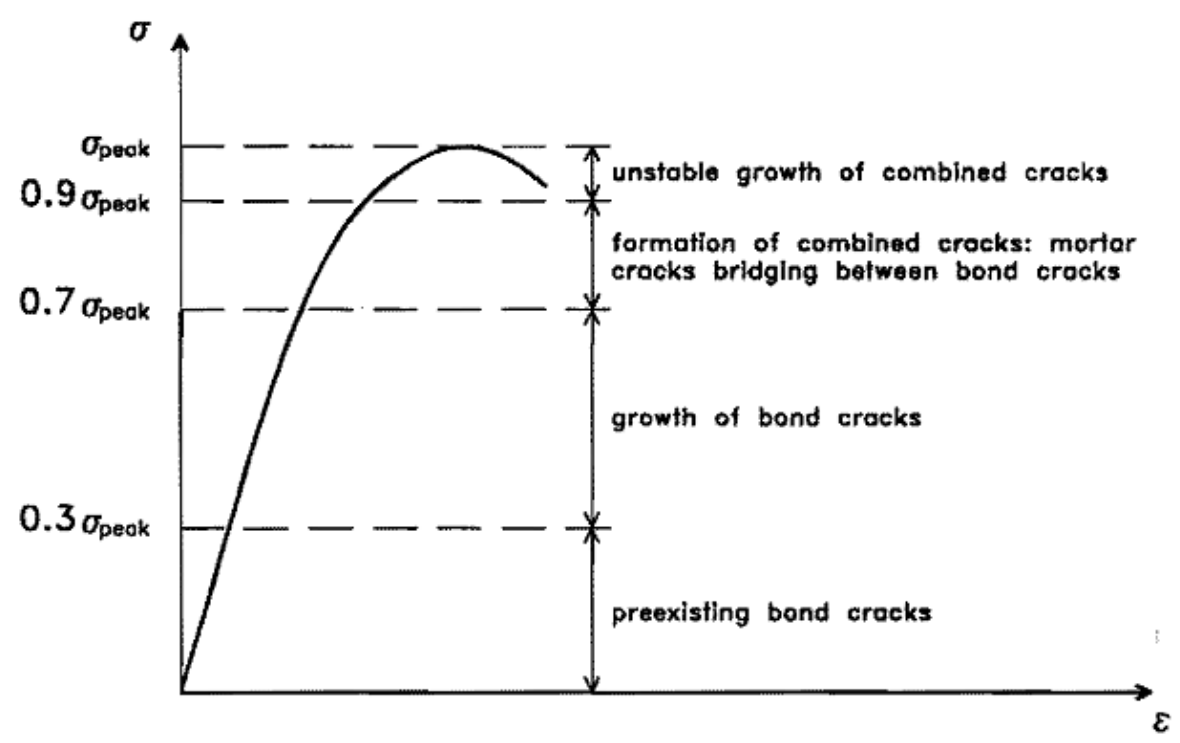

Figure 12 - Crack propagation in plain concrete under compression, from (HSU et al., 1963)

Besides the similar damage mechanisms developed before peak stress, UHPFRC presents a late nonlinear behavior. A straightforward way to determine the magnitude of the degradation mechanisms before the peak is by the ratio between initial modulus of elasticity $E_{0}$ and the secant modulus of elasticity $E_{s e c}$ that corresponds to the ratio between peak stress and peak strain. Then, the higher the nonlinear behavior before the peak, the more the ratio $E_{0} / E_{s e c}$. (GRAYBEAL, 2007) compared UHPFRC with steam curing $\left(f_{c}{ }^{\prime} \approx 193 \mathrm{MPa}\right)$ and a high strength concrete $\left(f_{c}{ }^{\prime} \approx 70 \mathrm{MPa}\right)$. The ratio $E_{0} / E_{\text {sec }}$ for UHPFRC was 1.1 and for HSC was 1.25 .

Furthermore, (GRAYBEAL, 2007) established a criterion to consider the beginning of the nonlinear behavior of UHPFRC under compression. According to the criteria, 
nonlinearity starts when the experimental stress value was reduced by $5 \%$ when compared to the value calculated with the initial modulus of elasticity at the same strain value, see Figure 13. For steam treated specimens of UHPFRC, the criterion was verified between 80 and $90 \%$ of the peak load. For normal strength concretes, this behavior is expected between $30 \%$ and $70 \%$ of the peak load, (HSU et al., 1963).

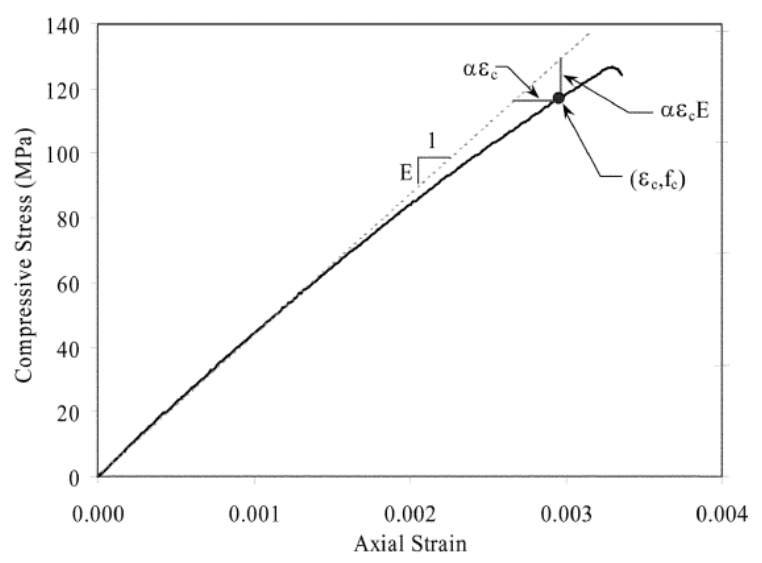

Figure 13 - Pre-peak behavior of UHPFRC, after (GRAYBEAL, 2007)

The nature of the fracture propagation processes and its energy demand under compression are the main factors that makes the concrete compressive strength much higher than tensile strength. Under tensile loading, the propagation is typically unstable because of only cohesion influences on strength. This type of fracture is commonly called mode I. For this reason, before cracking strength under tensile loading there is no detection of nonlinear behavior as opposed to concrete under compression.

For concretes with no coarse aggregates, as UHPFRC, the fracture processes are probably governed by the presence of pores (or concentrated porosities), air voids and microcracks distributed in the matrix, mainly at ITZ. Splitting cracks develop above and below the pores and the sand grains parallel to the load direction. The propagation of the cracks around such grains retains fast crack growth and consume more energy. Also, initial cracks that already exist in the composite propagates from the crack tips as wing cracks, as shown in Figure 14. 

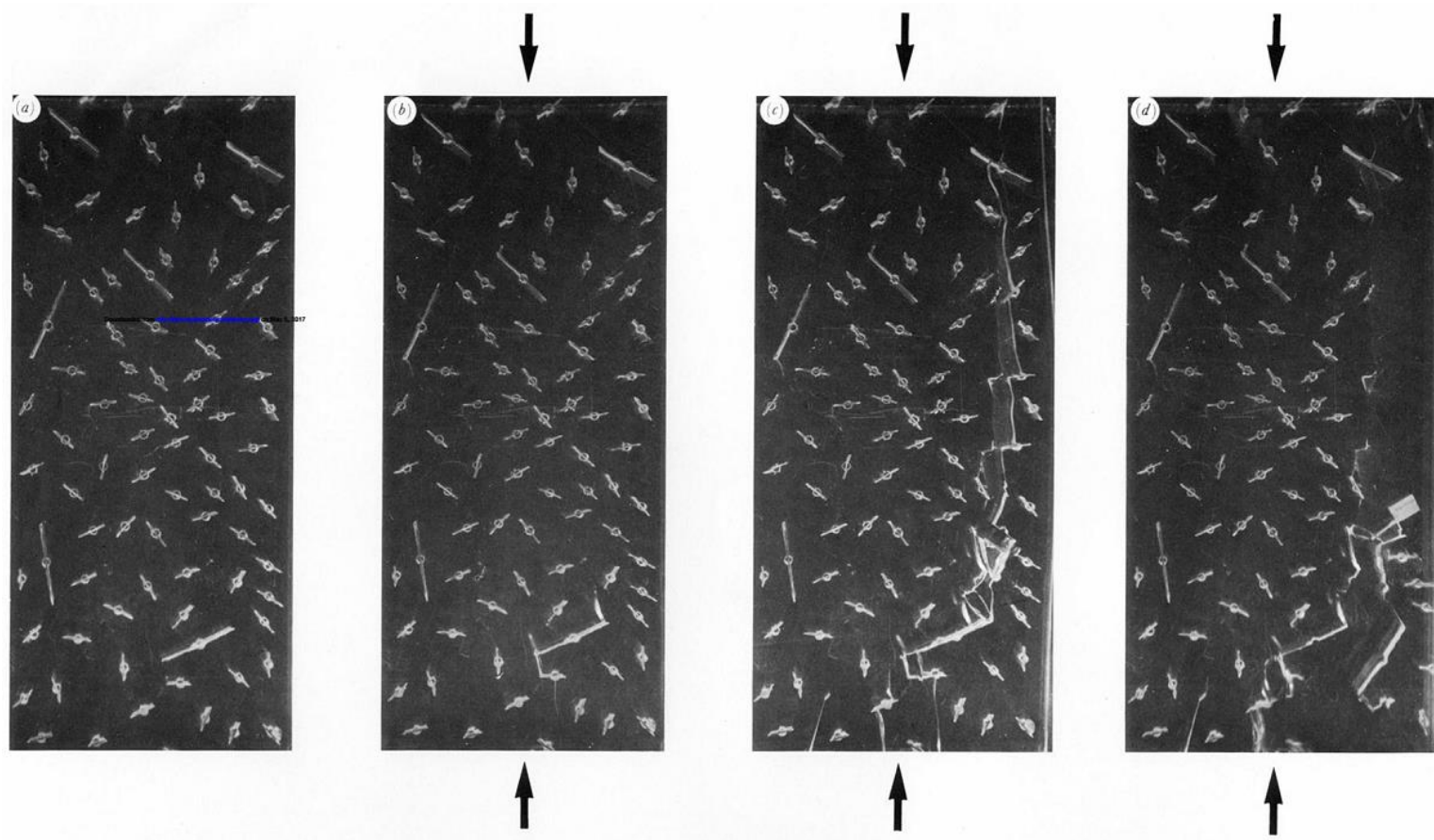

Figure 14 - Development of wing cracks under compression in solids with initial cracks, after

(HORII; NEMAT-NASSER, 1986)

These processes dissipate large quantities of energy in comparison to pure tensile crack propagation. As the crack tips are subjected to critical tensile stresses, even for lowstress levels, the crack propagates. For an inclined crack to the compressive load, the evolution of the crack tip tends to follow the direction of load, because of the transverse tensile strain. The crack propagation deviates from its initial orientation developing frictional slip between its faces and the process becomes more stable dissipating higher energy than in pure tension. These differences explain why compressive strength is higher than the tensile strength in cementitious materials. Figure 15 summarizes the discussed damage mechanisms occurred in concrete under compression. 


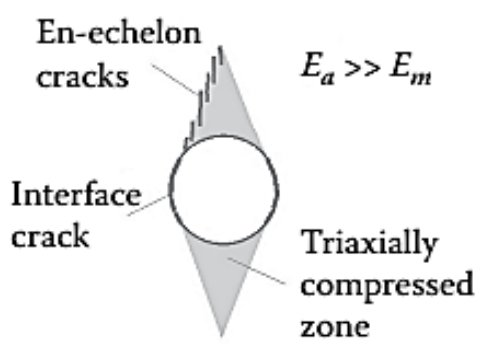

(c)

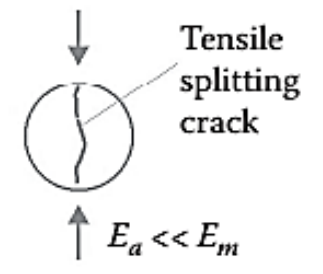

(d)

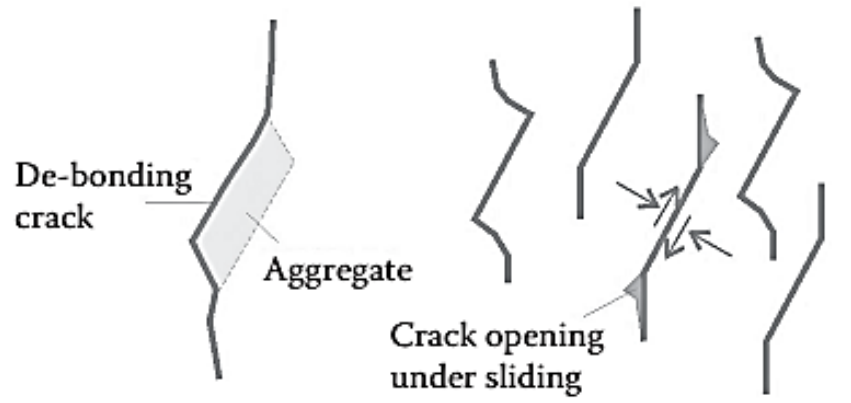

(f)

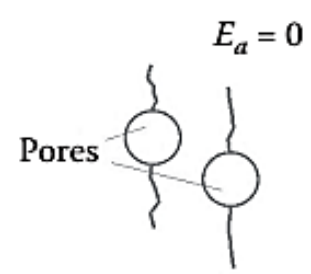

(e)

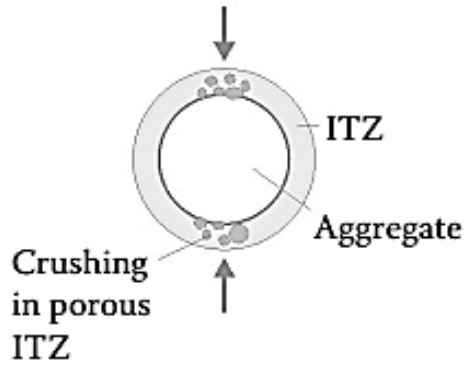

(g)

Figure 15 - Possible damage mechanisms that oooccurs in concrete under compressive stresses,

from (VAN MIER, 2012)

In the presence of fibers, the fiber-matrix interactions make the composite ductile and tough by bridging cracks. Such interacting mechanisms can be listed as physical and chemical adhesion, friction and mechanical anchorage. According to (BENTUR; MINDESS, 2007), the first two mechanisms can give significant contribution for composites with refined microstructure and low porosity and thereby a denser interfacial transition zone between fiber and matrix, as UHPFRC. This enhanced interaction is the main factor for the extensive use of straight fibers with surface treatment in UHPFRC that is one class of these advanced cementitious composites. Figure 16 presents a comparison of images from the fiber-matrix interfaces of high-strength fiber reinforced concrete and UHPFRC.

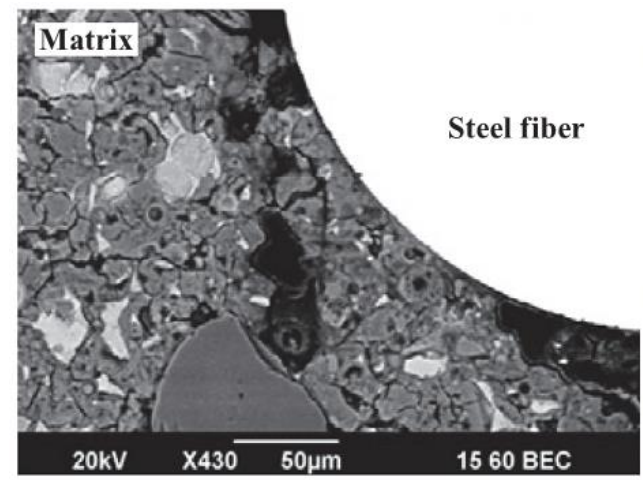

(a)

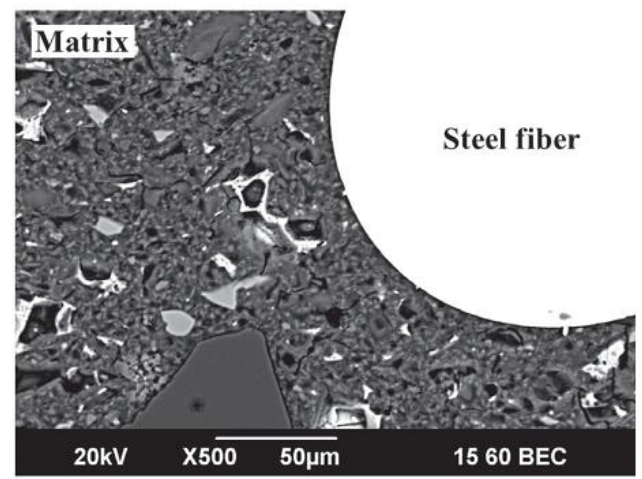

(b)

Figure 16 - Microscope image of (a) high-strength fiber-reinforced concrete, after (KIM et al., 2012), and (b) UHPFRC, after (YOO; PARK; KIM, 2017) 
The addition of fibers makes the fracture processes even more complex because the fibers action inhibits, at a certain level, the cracking propagation in plain concrete. Other dissipating mechanisms take place by fiber addition which governs the failure process and makes the composite ductile. Besides, early age microcracking can be developed from the ITZ around fibers, as presented by (WU; KHAYAT; SHI, 2017), Figure 17.

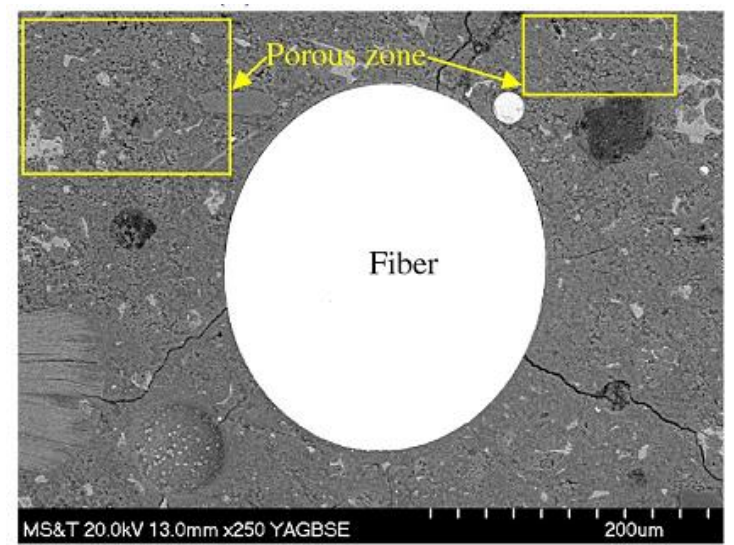

Figure 17 - Early age microcracks developed in UHPFRC, after (WU; KHAYAT; SHI, 2017)

Furthermore, there is some qualitative evidence that, under compression, the strain localization follows weak zones which pass through the interface between fiber and matrix typically parallel or slightly inclined to the direction of load. Some of this evidences was reported by $(\mathrm{OESCH}, 2015)$, which presented X-ray computed tomography images of UHPFRC specimens after being subjected to a compressive load, Figure 18.
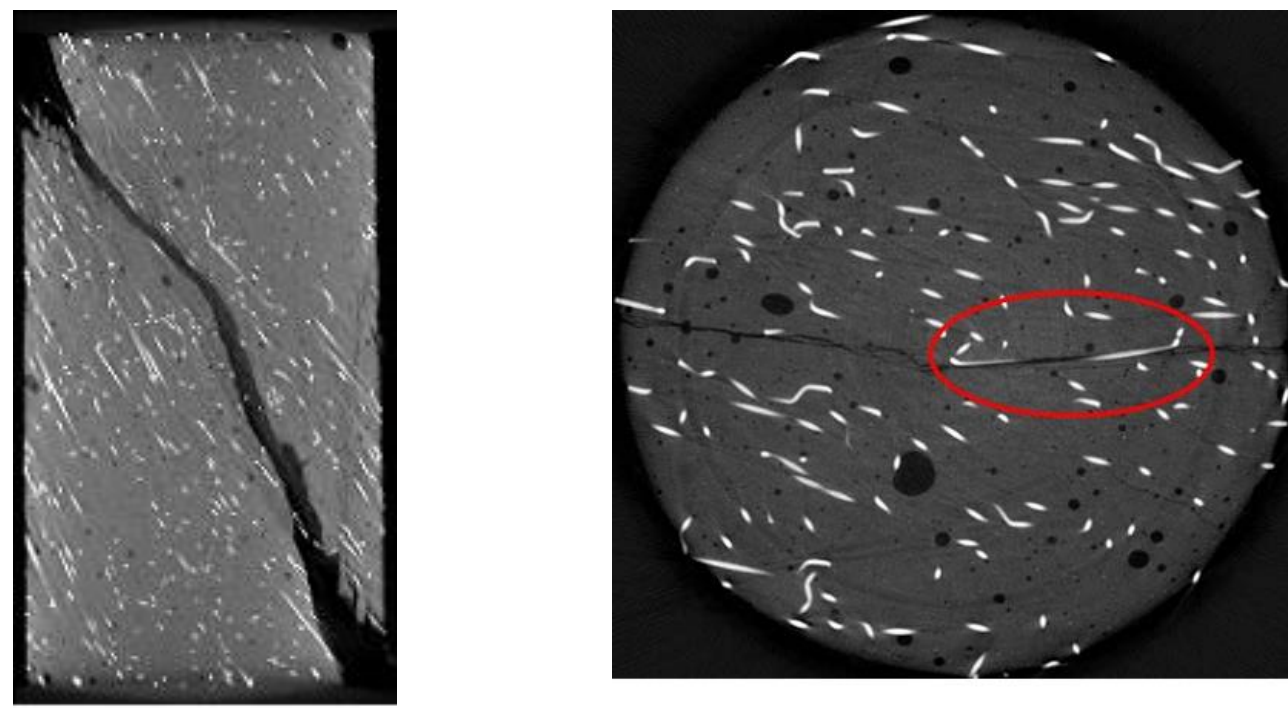

Figure 18 - Crack localization of UHPFRC under compression, after (OESCH, 2015)

After localization of strain near the peak stress, UHPC presents a brittle failure in the absence of fibers. In the presence of fibers, the composite exhibits a ductile behavior, and the fiber bridging also reflects in toughness that depends on the volume of fibers, type and 
aspect ratio. Furthermore, fiber hybridization tends to increase toughness. (EL-HELOU et al., 2016) presented experimental results of stress-axial strain and stress-radial strain showing the influence of fiber content, Figure 19.
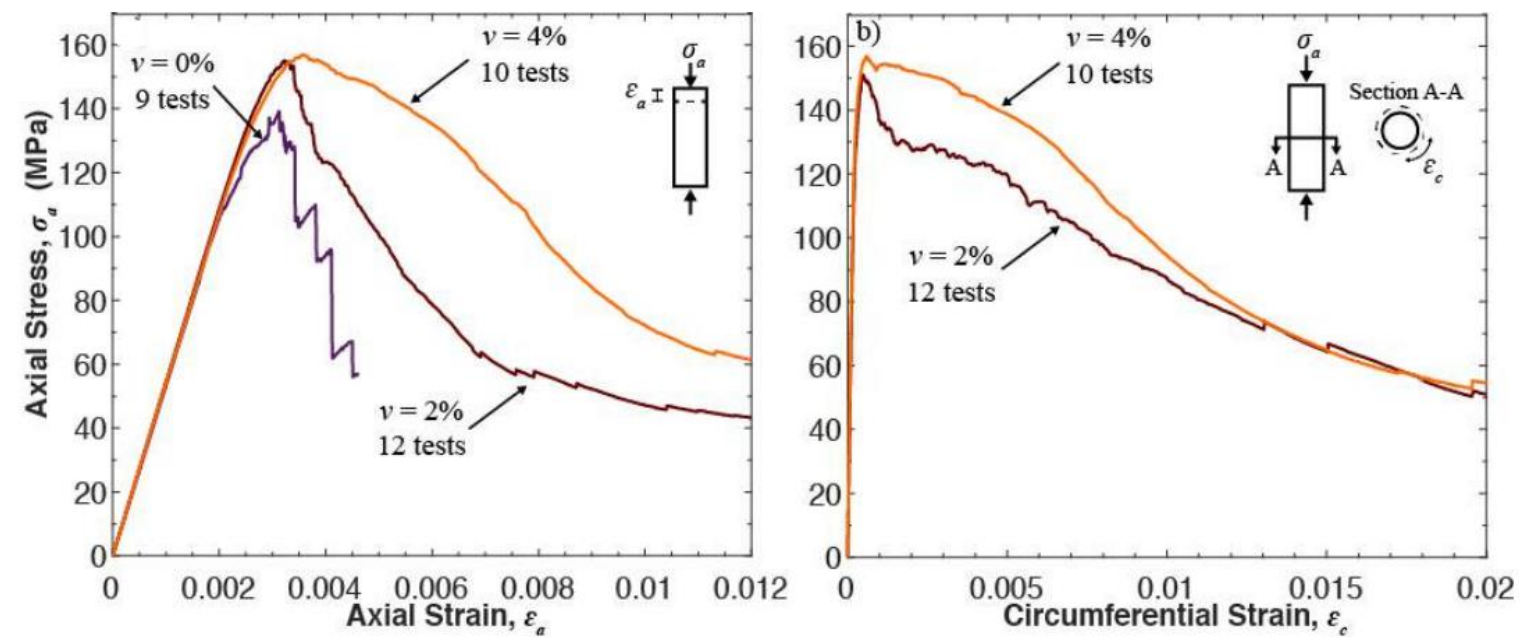

Figure 19 - Experimental compressive stress-strain results, after (EL-HELOU et al., 2016)

A tougher behavior was observed in both axial and radial stress-strain relations by increasing fiber content. Post-peak toughness can be attributed to the rotation of the main crack opening due to efficient bridging by fibers, that is, more fibers crossing a crack are more capable of inhibiting the crack opening because each fiber is less stressed. Another point is that the peak load increased from UHPC to the other fiber reinforced composites. The inclusion of fibers at the matrix delay the beginning of crack propagation. Therefore, the crack localization that occurs near the peak load occurs slightly later. Thus, the strength increase. However, a further increase in fiber content increased little the peak load when comparing UHPFRC with $2 \%$ and $4 \%$ of fibers. A possible explanation is that the required strain to the rise of the main crack near the peak will not change significantly with the further addition of fibers. Thus, the peak strength is not sensitive to this increase.

\section{Analytical stress-strain behavior}

The analytical equation that describes the uniaxial stress-strain curve of material is required for the design of structural elements. Through a code-oriented approach, the curve enables to obtain the nonlinear stress response for an applied strain directly. Besides, for a numerical method based on plasticity, the uniaxial stress-strain curve is commonly adopted as the hardening/softening evolution equation for the plasticity based concrete models.

Although several analytical equations have been proposed for concrete and fiber reinforced concrete (FRC), few studies were performed in developing and/or calibrate stress- 
strain equations for UHPFRC, (HARISH; DATTATREYA; NEELAMEGAM, 2013), (PREM, 2015) and (WU et al., 2016). Besides, it is known that the whole curve of FRC is challenging to represent through a single equation with the same constants calibrated for the ascending and descending branches. For this reason, some proposals defined constants to be calibrated for each range, as (FANELLA; NAAMAN, 1985), and others only for the descending branch (MANSUR; CHIN; WEE, 1999), (ZHOU et al., 2015). The fib Model Code uses differential equations to represent the ascending and descending branches of the stress-strain curve.

(DESAYI; KRISHNAN, 1964) proposed the analytical model, equation (8), that was further developed by (CARREIRA; CHU, 1985), which made the equation independent of calibration. Then, the equation was extended to equation (10) by (WEE; CHIN; MANSUR, 1996) and utilized by (MANSUR; CHIN; WEE, 1999) for HSFRC. Besides developed for concrete without fibers, the equation of (CARREIRA; CHU, 1985) is capable of predicting very well the pre-peak behavior of fiber reinforced concretes of different grades, inclusive UHPFRC. The proposed change by (MANSUR; CHIN; WEE, 1999) added two constants in the equation only for the post-peak branch to simulate the influence of the addition of fiber reinforcements to the matrix. The addition of these constants makes the function not continuous in the first derivative at peak.

The model of (CARREIRA; CHU, 1985) is characterized by the constant $\beta$, equation (8), which is determined by imposing boundary conditions to the curve that describes the nonlinear relation between stress and strain.

$$
\begin{aligned}
& f=f_{0}\left[\frac{\beta \frac{\varepsilon}{\varepsilon_{0}}}{\beta-1+\left(\frac{\varepsilon}{\varepsilon_{0}}\right)^{\beta}}\right] \\
& \beta=\frac{1}{1-\frac{f_{0}}{\varepsilon_{0} E_{i}}}
\end{aligned}
$$

Where $f_{0}, \varepsilon_{0}, E_{i}$ are the peak stress, peak strain and initial modulus of elasticity, respectively. The boundary conditions imposed to equation (8) to determine $\beta$ are 


$$
\begin{aligned}
& \left.\frac{d f}{d \varepsilon}\right|_{(\varepsilon=0)}=E_{i} \\
& \left.\frac{d f}{d \varepsilon}\right|_{\left(\varepsilon=\varepsilon_{0}\right)}=0 \\
& \left.f\right|_{\left(\varepsilon=\varepsilon_{0}\right)}=\sigma_{0}
\end{aligned}
$$

For the post-peak toughness of fiber-reinforced concrete, (MANSUR; CHIN; WEE, 1999) utilized the equation (10) to represent the behavior of HPFRC.

$$
\begin{aligned}
& f=f_{0}\left[\frac{k_{1} \beta \frac{\varepsilon}{\varepsilon_{0}}}{k_{1} \beta-1+\left(\frac{\varepsilon}{\varepsilon_{0}}\right)^{k_{2} \beta}}\right] \\
& k_{1}=\left(\frac{50}{f_{0}}\right)^{3}\left[1+2.5\left(\chi_{f}\right)^{2.5}\right] \\
& k_{2}=\left(\frac{50}{f_{0}}\right)^{1.3}\left[1-0.11\left(\chi_{f}\right)^{-1.1}\right]
\end{aligned}
$$

Where $k_{1}$ and $k_{2}$ are empirical constants dependent on the peak stress and fiber factor $\chi_{f}$

\subsection{Biaxial and triaxial behaviors}

Cement-based materials are sensitive to biaxial and triaxial stress states. For UHPFRC, experimental results in this field are few. Relevant research was developed by (SPECK, 2008), which reported results for high-performance concrete (HPC) and UHPFRC with different fiber contents under uniaxial, biaxial, triaxial stress states. The Lubliner criterion is presented with the experimental results. This function will be further discussed in the next section. Equation 11 represents the non-triaxial compression region for Lubliner function, as derived by (ZHANG; LI, 2012). For the compressive meridian, the lode angle is $\theta=\pi / 3$, and for the tensile meridian, the angle is $\theta=0$. The Lubliner function was written as a function of the polar distance $\rho$, the hydrostatic coordinate $\xi$ and the Lode angle $\theta=0$.

$$
\begin{aligned}
& \left(\sqrt{3} \alpha+\frac{\beta}{\sqrt{3}}\right) \xi+\left(\sqrt{\frac{2}{3}} \beta \cos \theta+\sqrt{\frac{3}{2}}\right) \rho-(1-\alpha) \frac{f_{c}\left(\varepsilon^{p}\right)}{f_{c}}=0, \\
& 0 \leq \theta \leq \frac{\pi}{3}
\end{aligned}
$$


The triaxial compression region is expressed by equation (12).

$$
\begin{aligned}
& \left(\sqrt{3} \alpha+\frac{\gamma}{\sqrt{3}}\right) \xi+\left(\sqrt{\frac{2}{3}} \gamma \cos \theta+\sqrt{\frac{3}{2}}\right) \rho-(1-\alpha) \frac{f_{c}\left(\varepsilon^{p}\right)}{f_{c}}=0, \\
& 0 \leq \theta \leq \frac{\pi}{3}
\end{aligned}
$$

The parameter $\beta$ is determined based on the inputted hardening/softening evolution law for tension and compression (LEE; FENVES, 1998), see equation 13.

$$
\beta\left(\tilde{\varepsilon}_{c}^{p}\right)=(1-\alpha) \frac{f_{c 0}\left(\tilde{\varepsilon}_{c}^{p}\right)}{f_{t}\left(\tilde{\varepsilon}_{c}^{p}\right)}-(1+\alpha)
$$

Parameter $\alpha$ is obtained from the relation between initial yield compressive stress $f_{c 0}$ and biaxial initial yield compressive stress $f_{b c 0}$

$$
\alpha=\frac{f_{b c 0} / f_{c 0}-1}{2 f_{b c 0} / f_{c 0}-1}
$$

Parameter $\gamma$ is obtained from $K_{c}$ constant that governs the spacing between compression and tension meridians and the section shape of yielding surface on the deviatory plane, equation 15 . However, this is the only parameter calibrated with triaxial test results. The lower and upper limits of $K c$ are 0.5 and 1.

$$
\gamma=\frac{3\left(1-K_{c}\right)}{2 K_{c}-1}
$$

The behavior of each meridian is divided into two parts because of the singularity function, which will be presented in the next section. The invariants $\rho$ and $\xi$ are presented in equations (16) and (17), respectively.

$$
\begin{aligned}
& \rho=\frac{\sqrt{2 J_{2}}}{f_{c}} \\
& \xi=\frac{I_{1}}{\sqrt{3} f_{c}}
\end{aligned}
$$

Different from steel, if the experimental result is plotted in the octaedral plane, concrete is sensitive to differential triaxial stress states, that is, is sensitive to Lode angle. Thus, the behavior of normal strength concrete (NSC), HPFRC and UHPFRC are represented in the octaedral plane with equations (11) and (12). For NSC, experimental results of uniaxial compression tests with active confinement of (SFER et al., 2002) and the equibiaxial compressive strength from (KUPFER; HILSDORF; RUSCH, 1969) are plotted. For HSFRC, uniaxial compression test results with active confinement of (CANDAPPA; 
SANJAYAN; SETUNGE, 2001) and triaxial and equibiaxial test results of (SPECK, 2008). For UHPFRC, triaxial and equibiaxial test results for UHPFRC of (SPECK, 2008) and uniaxial compression tests with active confinement (REN et al., 2016). The parameter $K_{c}$ of Lubliner function presented governs its shape on the octahedral plane. From the hydrostatic stress state correspondent of the test results, the parameter $\xi$ is fixed. Thus through equations (11) and (12), the deviatoric stress $\rho$ is determined as a function of the lode angle $\theta$.

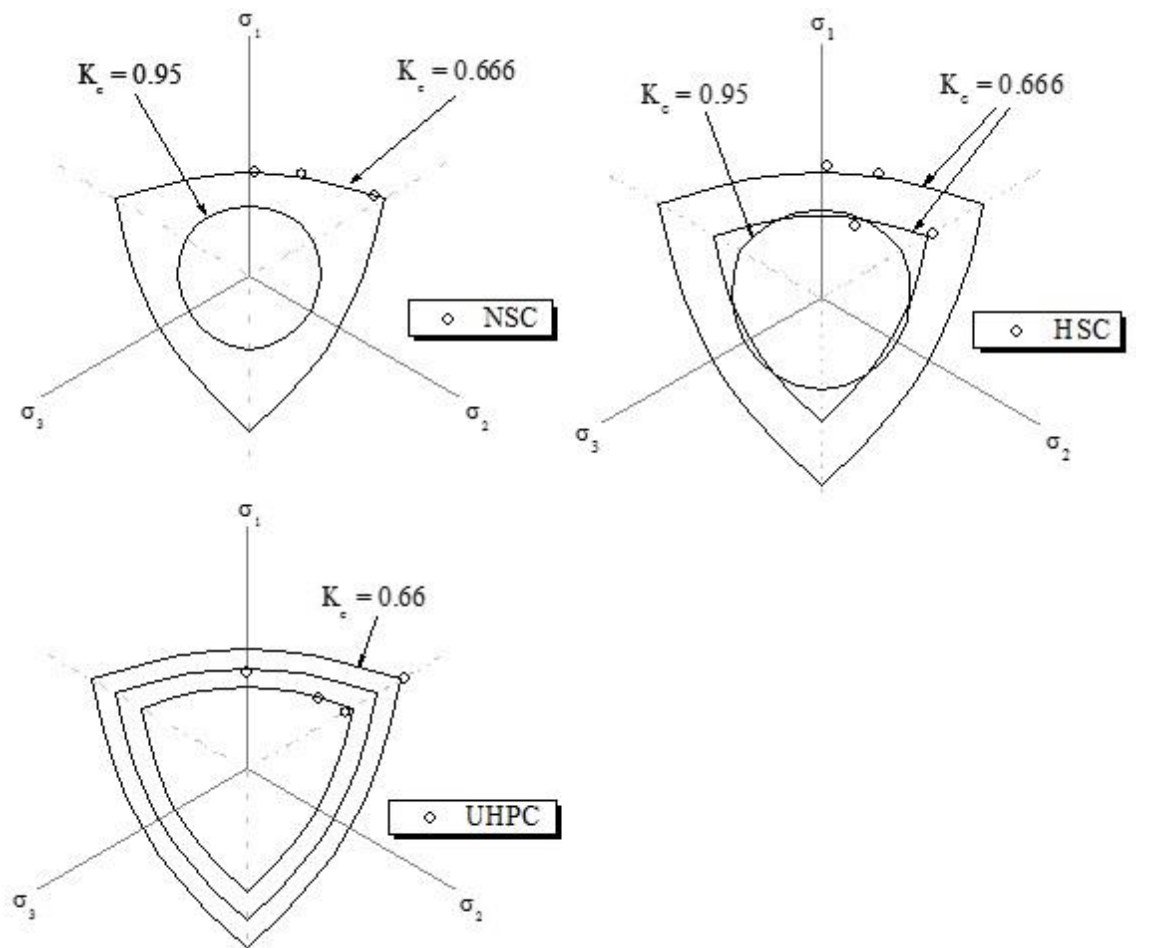

Figure 20 - Lubliner surface in the deviatoric plane

In all situations presented in Figure 20, the best approximation was obtained by taking $K_{c}$ equal 0.666 . For this value near 1 , the Lubliner function reduces to the DruckerPrager criteria.

Under equibiaxial compressive stress states $\sigma_{1}=\sigma_{2}$, concrete presents higher strength than in uniaxial compressive states. Figure 21 shows experimental results of biaxial compression for NSC, HPC, and UHPC, performed by (SPECK, 2008) that are compared to the Lubliner yield criterion. 


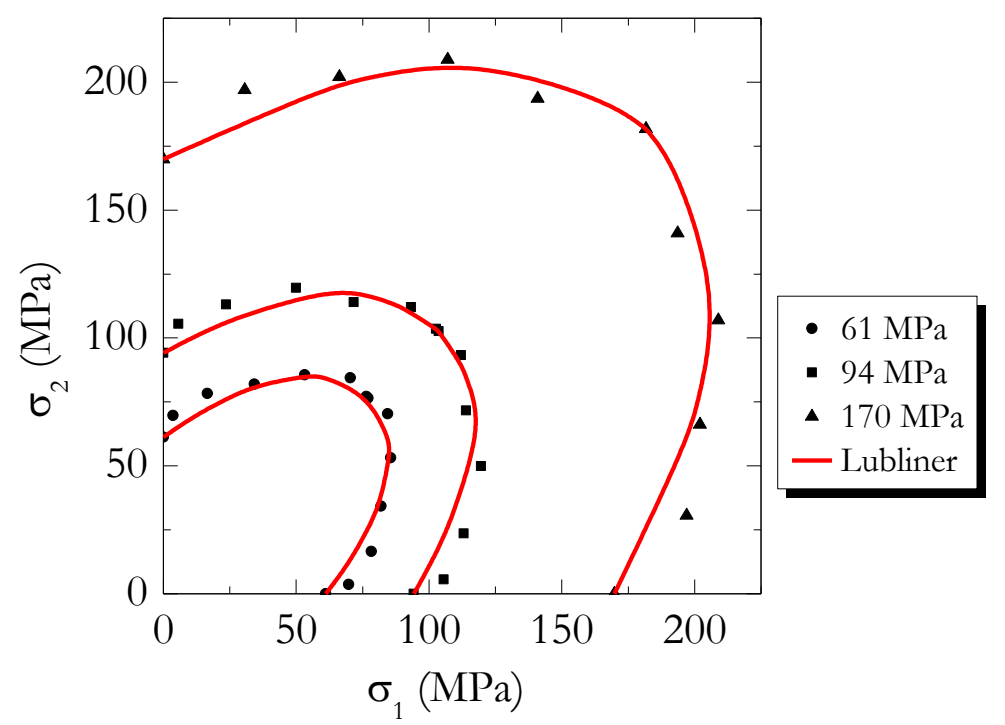

Figure 21 - Lubliner yield criterion and biaxial compression experimental results of (Speck 2008).

From Figure 21, the ratio between equibiaxial and uniaxial strengths $f_{b c} / f_{c}$ can be obtained for the presented concrete classes. For NSC, the ratio is $f_{b c} / f_{c}=1.26$, for HPC with $94 \mathrm{MPa}$ is $f_{b c} / f_{c}=1.1$ and for UHPC with $170 \mathrm{MPa}$ is $f_{b c} / f_{c}=1.07$. Thus, it can be concluded that the relation $f_{b c} / f_{c}$ decreases as the compression strength increases.

\subsection{Concrete Damage Plasticity constitutive model for UHPFRC}

The constitutive model to represent concrete behavior must try to incorporate some microscopic features experimentally observed, such as microvoids consolidation or propagation of microcracks, (MAZARS, 1986), to describe the macroscopic behavior of the material. The description of these irreversible processes, as plastic deformation and rigidity decrease (damage), can be made by a model that couples damage evolution to the framework of plasticity models, (LUBLINER et al., 1989) and (LEE; FENVES, 1998) and (GRASSL; JIRÁSEK, 2006). Thus, concrete and more specifically UHPFRC are modeled as a continuous isotropic media with the material behavior governed by Damage-Plasticity model.

Conventionally, the ductile behavior of materials is represented by the evolution of the yielding surface. This evolution is described by the uniaxial compressive/tensile behavior of the material, which is called of hardening/softening evolution laws. These equations will be proposed in the present research. Furthermore, the calculus of stiffness degradation of structures is governed by the damage evolution laws. Such laws are also proposed in this work. 
The constitutive model utilized to simulate UHPFRC is based on the effective stress concept, (LEMAITRE; CHABOCHE, 1990). Equation (18) presents the yielding criteria, (LUBLINER et al., 1989) and (LEE; FENVES, 1998), applied to simulate the nonlinear behavior of the composite. Equation (19) shows the model parameters.

$$
\begin{aligned}
& f(\bar{\sigma}, \kappa)=\frac{1}{1-\alpha}\left[\bar{q}-3 \alpha \bar{p}+\beta(\kappa)\left\langle\bar{\sigma}_{\max }\right\rangle-\gamma\left\langle-\bar{\sigma}_{\max }\right\rangle\right]-\bar{\sigma}_{c}(\kappa) \\
& \alpha=\frac{f_{b c 0} / f_{c 0}-1}{2 f_{b c 0} / f_{c 0}-1} ; \quad \beta\left(\varepsilon^{p}\right)=(1-\alpha) \frac{f_{c 0}\left(\varepsilon_{c}^{p}\right)}{f_{t}\left(\varepsilon_{t}^{p}\right)}-(1+\alpha) ; \quad \gamma=\frac{3\left(1-K_{c}\right)}{2 K_{c}-1}
\end{aligned}
$$

Where, $\langle\bullet\rangle$ is the Macauley bracket, $\kappa$ is the hardening/softening function which corresponds to the plastic strain, as presented in equation (19), $\bar{q}=\sqrt{\frac{3}{2} \bar{S}: \bar{S}}$ the effective von Mises equivalent stress, $\bar{p}=-\frac{1}{3} \bar{\sigma}: I$ effective hydrostatic stress, $\bar{\sigma}_{\max }$ the algebraically largest eigenvalue of the effective stress tensor, $f_{c 0}$ yield compressive stress, $f_{b c 0}$ equibiaxial compressive stress strength, $f_{c 0}\left(\varepsilon_{c}^{p}\right)$ uniaxial compressive stress-plastic strain curve, $f_{t}\left(\varepsilon_{t}^{p}\right)$ uniaxial tensile stress-plastic strain curve and $K_{c}$ is a constant that governs the distance between compressive and tensile meridians and the shape of failure surface on deviatory plane. The lower and upper limits of $K_{c}$ are 0.5 and 1 . The compressive meridian corresponds to the intersection curve between the failure surface and a plane containing the hydrostatic axis with a Lode angle of 60 degrees. For the tensile meridian, the lode angle is 30 degrees.

The initiation of the inelastic process affects the evolution of the surface that limits the elastic domain, equation (18). The functions describing the evolution of the yielding surface were adopted as the uniaxial responses of UHPFRC under tension, equation (58), and compression, equation (99).

Furthermore, non-associated plasticity is considered to calculate plastic strains. In the current model, the intensity and directions of plastic strains derive from the plastic potential function that is a Drucker-Prager type function:

$$
G=\sqrt{\left(m f_{t} \tan \phi\right)^{2}+q^{2}}+p \tan \phi
$$

Where $\phi$ is the dilation angle, $m$ is called eccentricity, $q$ is the effective von Mises stress invariant and $p$ hydrostatic stress invariant. 


\subsection{Calibration of the CDP model}

The parameters of the plastic potential function and yielding function were calibrated according to experimental results with UHPC reported in the literature. Figure 22 and Figure 23 compare triaxial and biaxial test results taken from literature, (SPECK, 2008), (FARNAM et al., 2010), (ZOHREVAND; MIRMIRAN, 2011), (SOVJÁKA; VOGEL; BECKMANN, 2013) (REN et al., 2016), and the adopted failure surface represented in the HaighWestergaard plane as function of the stress invariants $\xi=I_{1} / \sqrt{3}$ and $\rho=\sqrt{2 J_{2}}$. Parameters $\alpha$ and $\beta$ are determined for peak tensile and compressive stress strengths and equibiaxial compressive strength. The parameter $K_{c}$ was adopted as 0.66 to best fit experimental results in the octahedral plane, Figure 23.

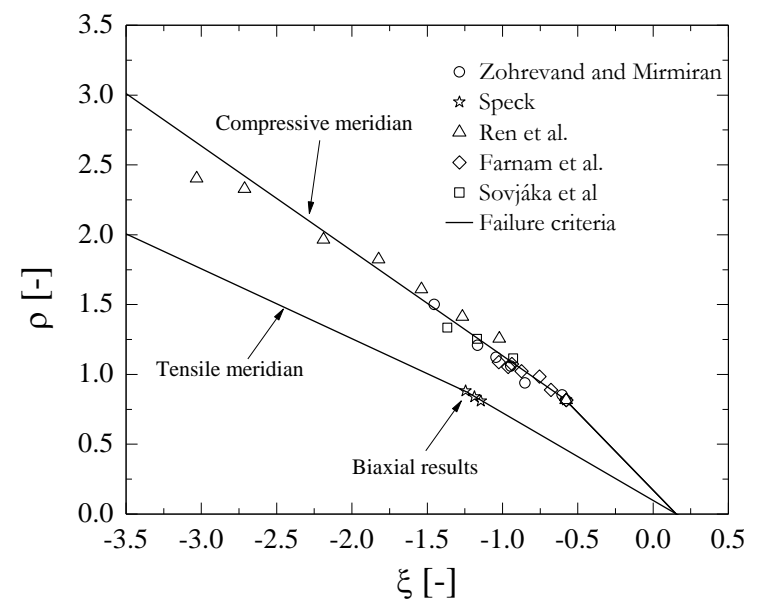

Figure 22 - Comparison between meridians and experimental results

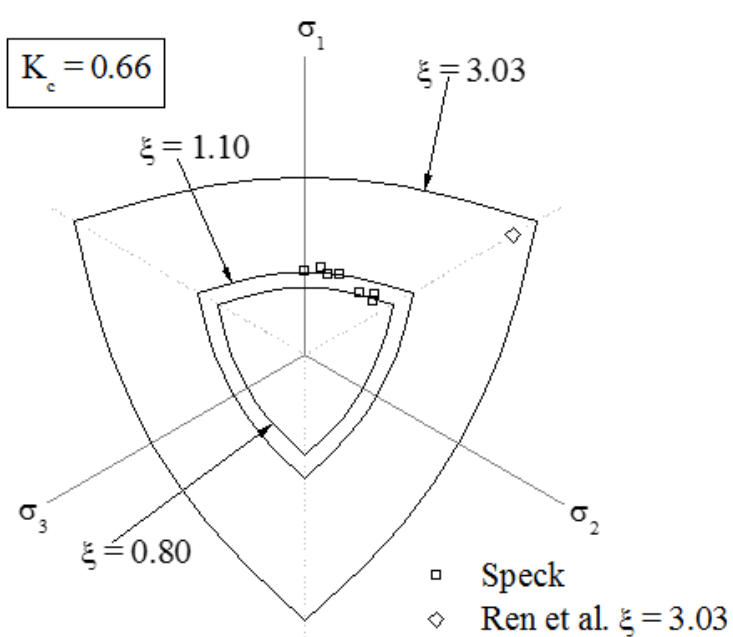

Figure 23-Failure envelopes in the deviatoric plane for different hydrostatic stress states

Figure 24 presents equibiaxial and nonequibiaxial experimental results of (SPECK, 2008) (compression-compression tests) and (LEE et al., 2017a) (tension-compression tests) compared to the calibrated failure envelopes of the Concrete Damage Plasticity model (CDP) in the plane of principal stresses $\sigma_{1}$ and $\sigma_{2}$. The uniaxial compressive strength normalizes the experimental results. Figure 25 presents the plastic-potential function in the space of the hydrostatic $p$ and deviatoric $q$ stress invariants. The surface is compared to triaxial compressive experimental results. According to the model, the inclination of a straight line that passes through this experimental data corresponds to the dilation angle $\phi$. 


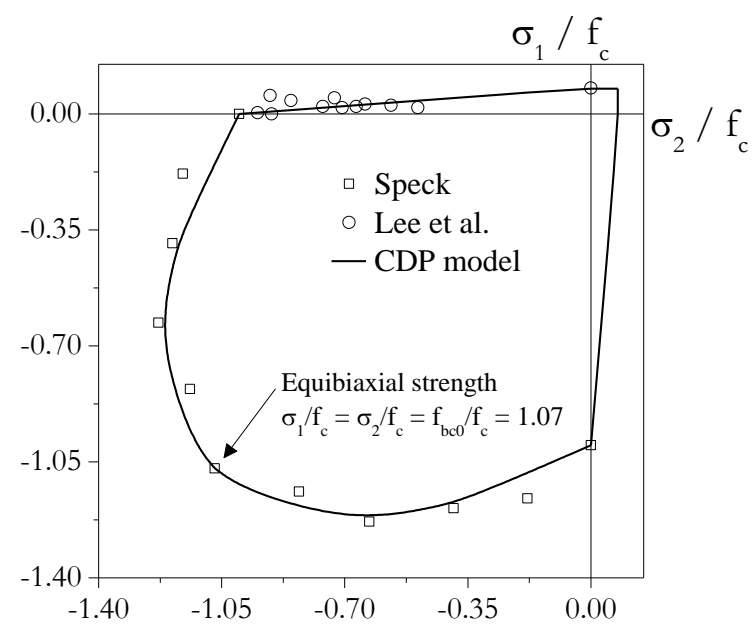

Figure 24 - Biaxial failure envelopes compared to experimental results

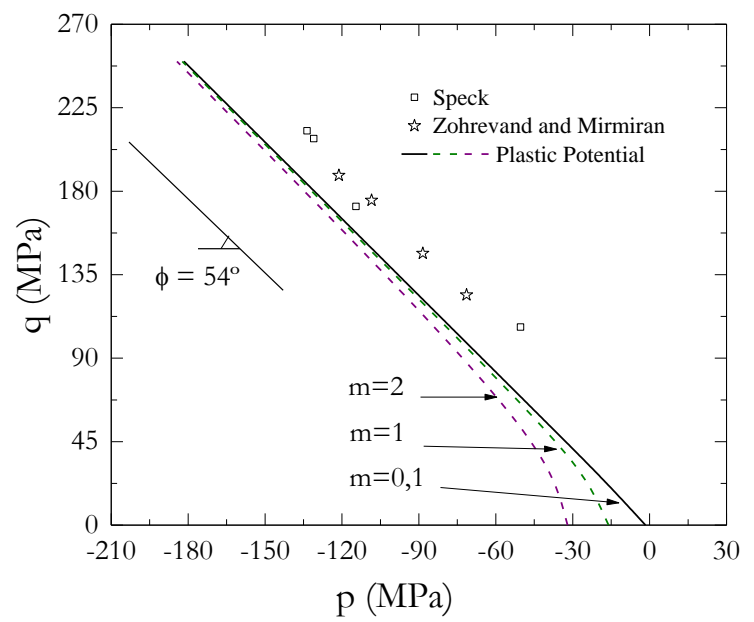

Figure 25-Plastic potential function

The eccentricity parameter $m$ and the viscosity parameter $\mu$ were adopted respectively as 0.1 and 0.0001 , (LI; HAO; BI, 2017). The parameter $m$ governs the shape of the plastic potential function for low hydrostatic stress states, as shown in Figure 25. The parameter $\mu$ is a mathematical tool to decrease the mesh sensitivity for constitutive models that undergoes softening behavior. As this parameter increases the damage caused by the mechanical load is distributed in a greater area, that is, the damage is not localized in the integration point. Table 5 presents the parameters that define the Lubliner surface calibrated for UHPFRC.

Table 5- Plasticity parameters of the Plastic-Damage model for UHPFRC

\begin{tabular}{cc}
\hline$\phi$ & $54^{\mathrm{o}}$ \\
$m$ & 0.1 \\
$f_{b c 0} / f_{c 0}$ & 1.07 \\
$K_{c}$ & 0.666 \\
$\mu$ & 0.0001 \\
\hline
\end{tabular}

The viscosity parameter $\mu$ is a mathematical resource that tries to overcome the mesh dependency of models that incorporate strain softening to the constitutive model. The basic idea of $\mu$ is to distribute or homogenize the localized damage at an integration point to an influence region, which agrees with what happens in cement-based composites. Thus, the lower the viscosity parameter $\mu$, the higher is the damage influence area. The consequence of the better distribution of damage is the increase in toughness of the numerical response. 


\section{STATE-OF-THE-ART OF LATERAL STABILITY OF CONCRETE BEAMS IN TRANSITORY PHASES}

Precast concrete beams are susceptible to transitory stages of construction since demolding from formwork until the permanent phase. All these stages are described in (EL DEBS, 2017), which highlights the possibility of different efforts from the service stage and the importance of concrete strength at early ages. The phases that present primary concern with structural stability of beams are lifting, transport and beam supported only by bearing pads. Several published research discuss on which is the most critical. However, all must be considered as critical due to several accidents reported on each one (scientific journals, technical reports and on the internet, including videos). Figure $26(\mathrm{a})^{1}$ and (b) ${ }^{2}$ are accidents with beams during lifting and transport, reported by online newspapers, and Figure 26 (c) is an accident with beams supported by bearing pads that were reported by (HURFF; KAHN, 2012a).

\footnotetext{
${ }^{1}$ http://jornaldesantacatarina.clicrbs.com.br/sc/geral/noticia/2018/02/acidente-com-viga-forca-asuspensao-de-trabalhos-em-trecho-da-duplicacao-da-br-470-em-gaspar-10156845.html ${ }^{2}$ http://www.oregonlive.com/portland/index.ssf/2013/04/tractor_trailer_crash_on_marqu.html
} 


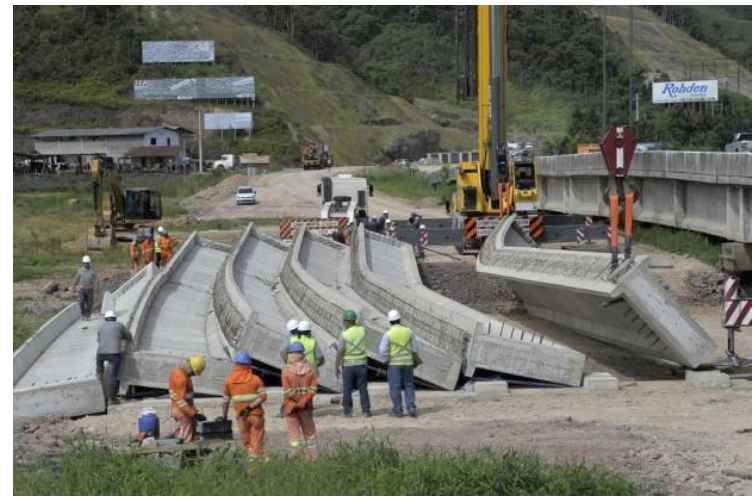

(a)

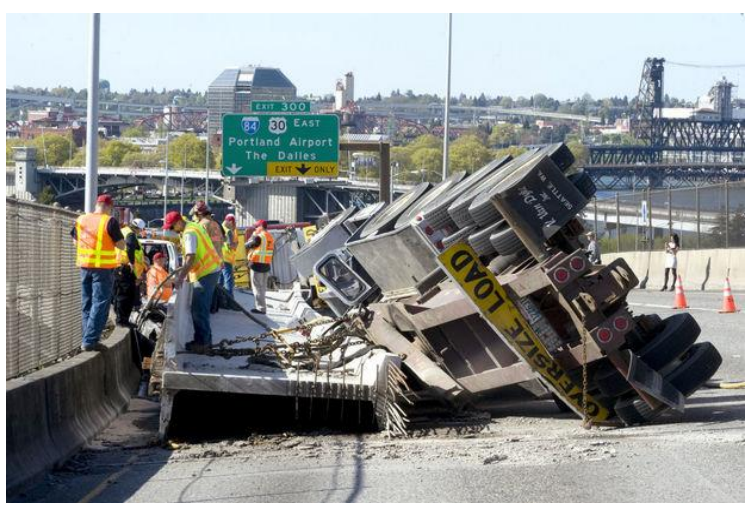

(b)

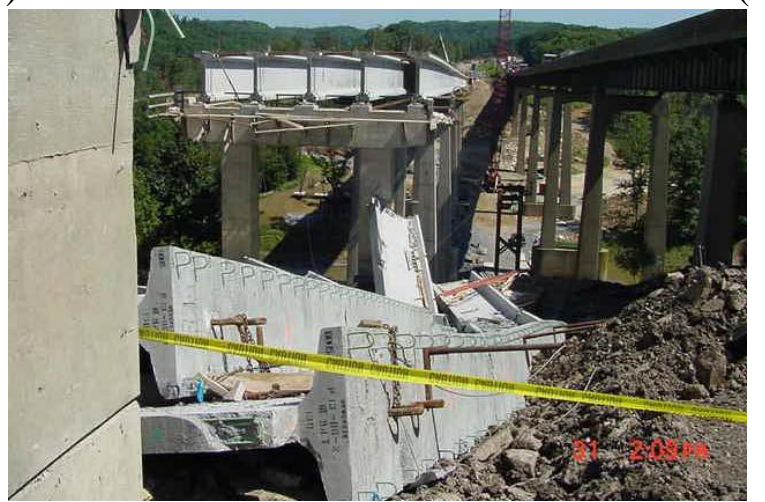

(c)

Figure 26 - Accidents occurred during transitory phases of construction

There are no scientific studies about these cases. However, possible causes can be raised. The first hypothesis that can be pointed out is the existence of initial imperfections which arise due to several factors as creep, shrinkage, manufacturing errors, thermal loading, differential prestress forces between cables and others. Besides, almost every study concerning instability problems concludes the significant influence of these parameters. Also, the concrete age and consequently the developed modulus of elasticity has a significant impact in all stages, because beams with greater lateral stiffness are less prone to instability.

Furthermore, each transitory situation has particularities mainly concerning the boundary conditions and even the mechanical behavior. These particularities must be considered on the analytical developments. For example, concrete beams during lifting only present stable equilibrium when its center of mass cross the roll axis at the points were the lifting cables are attached, see Figure 27. 


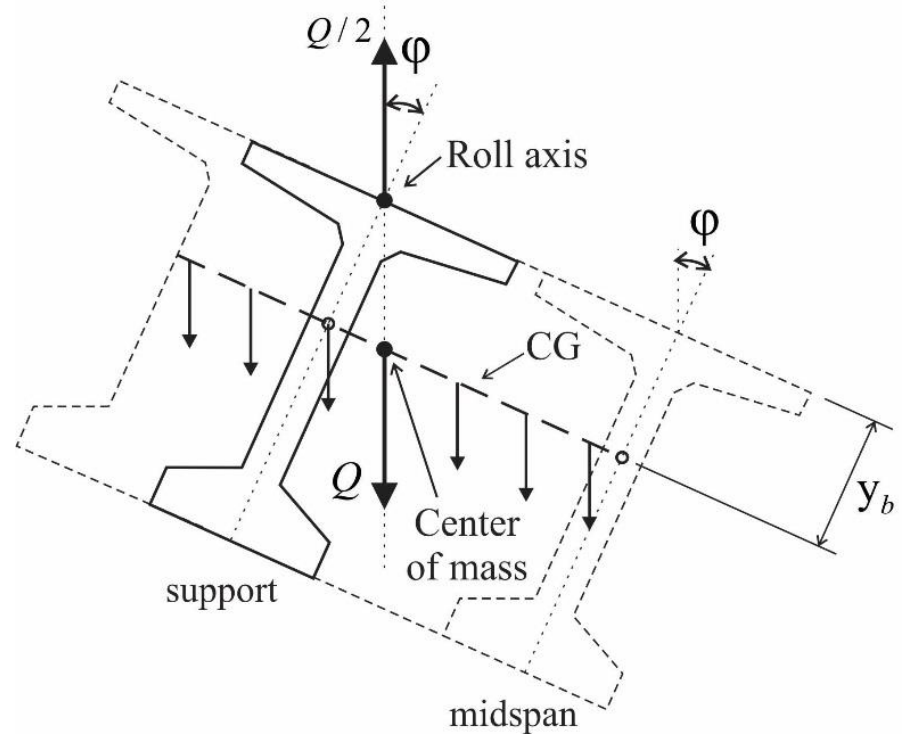

Figure 27 - Precast beams lifted by cables

This condition is required because the supports at lifting point do not present any restriction for the beam rotation. Thus, imperfect beams always present initial rotation for equilibrium, as was observed in the experiments of (SWANN; GODDEN, 1966). Besides, in the lifting problem the further variables also influence on beam stability:

- The longitudinal position of the lifting cables (overhangs);

- The vertical position of the lifting cables, $y_{b}$;

- The inclination of the cables;

- The eccentricity of the lifting points;

- Dynamic effects due to crane movements;

- Increased lateral displacements and rotation due to wind load.

The first two factors are design parameters and as is well known the increase of overhangs length for the attachment points can increase the stability capacity of the beam substantially, (MAST, 1989; STRATFORD; BURGOYNE, 2000). Also, the increase in the height of the lifting points $y_{b}$ relative to the centroid improves the beam stability. The inclination of the cables reduces the capacity of lateral stability of the beams due to the horizontal compressive load that arises. Dynamic effects can be avoided if the crane operations were carried carefully and in days with low-velocity winds.

Beams under transport and supported by bearing pads have similar mechanical behavior. The supports provide a restriction to rotations. Thus, the beam can present stable equilibrium even when the center of mass does not cross the roll axis, which in these cases pass below the bottom flange of the beam at supports, see Figure 28. 


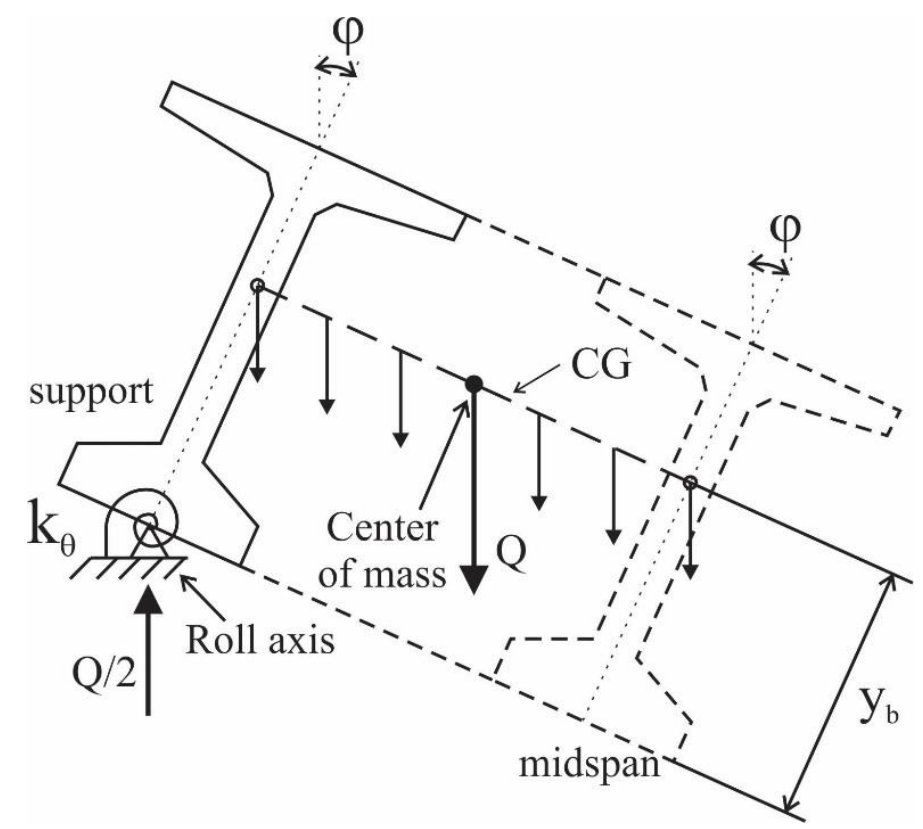

Figure 28 - Precast beam supported at the bottom face

The restriction provided by the supports is commonly taken in analytical solutions as a rotational spring $k_{\theta}$ relative to longitudinal rotations. This spring stiffness governs the stability of the beams and is accounted in equilibrium solutions, (MAST, 1993) and (BURGOYNE; STRATFORD, 2001). (MAST, 1993) recommends values of $k_{\theta}$ for the transportation equipment (truck and trailer) which will be briefly discussed later. After erected, the beam is only supported by bearing devices. Several types of bearings are used in precast structures, but the most common is the elastomeric bearing pad, which can be plain or steel reinforced. As shown experimentally by (CONSOLAZIO; HAMILTON, 2012), when the elastomeric pads carry an axial load and a subsequently applied moment, the behavior is nonlinear, due to the material, but also due to the partial loss of contact "liftoff' with the beam bottom flange. This effect can be more dangerous due to the lack of flatness of the beam bottom surface. The decrease of the contact area reduces the rotational stiffness of the pad. (KRAHL; CARRAZEDO; EL DEBS, 2017) recommended the utilization of the secant stiffness of the nonlinear moment-rotation behavior of the pad to account for lift-off in predicting the load capacity of beams.

Besides the particularities about the torsional stiffness of the supports for each of these phases, during transport, the beam stability is also influenced by:

- Superelevation of the road;

- Dimensions of the truck and trailer (axis of rotation);

- Dynamic effects;

- Assembly errors in positioning the beam on the vehicle. 
(LASZLO; IMPER, 1987) utilized for the verification of beam stability during transport a superelevation of $8 \%$. In Brazil, a superelevation of $10 \%$ is permitted. This initial condition causes further displacements due to the flexibility of the truck and trailer. Furthermore, as is known, dynamic effects can increase the displacements of structures, which can be critical when the vehicle is on a superelevation. However, from the knowledge of the author, there is one recent study that investigated this stage, (MCGORMLEY; LINDENBERG, 2016), and is out of the scope of the present research. Then, the topic is recommended for future work.

The stability of beams supported by bearing pads is also influenced by:

- Increase in lateral displacements by solar radiation and lateral wind loads;

- Assembly errors in positioning the beams on bearing pads;

- Lack of flatness of the bottom flange;

It is common in practice, although non-recommended, leaving the beams exposed several days to solar radiation and wind, with provisory bracings, sometimes, insufficient. These additional effects can be critical for a collapse. (HURFF, 2010) investigated the influence of solar radiation on the increase of lateral displacements and observed that thermal effects could increase these imperfections by $40 \%$. Furthermore, (HURFF; KAHN, 2012b) reported the influence of the lack of flatness on the stability. The beam presented a much lower capacity than expected with high initial rotations. Then, the bottom face was retrofitted, and the capacity of the beam was reestablished. Also, errors in positioning the beam on the bearing pads can influence the rotational stiffness of the pad. It is worth mentioning that this subjects must be further studied mainly to determine tolerances.

This field of research has been explored, but, as opposed to the number of reviews available for UHPFRC presented in item 2.1, there is no review about the lateral instability of concrete beams in transitory phases. Thus, an effort of the present research is to cover some gaps in the field and provide a review.

\subsection{Review of design guidelines}

An important topic is about code recommendations. There are some discussions about the unsafety recommendations of the main codes available for design. A recent study on its validity was provided by (DE LA FUENTE ANTEQUERA; CAVALARO; GARCÍA, 2017). The authors concluded that the only code that presents a safe recommendation for their case study was the Spanish EHE-08 (2009). This conclusion was opposed to the 
findings of the research presented by (KRAHL; LIMA; EL DEBS, 2015), which recommended the verification of (FIB MODEL CODE, 2010) as a safe one. (DE LA FUENTE ANTEQUERA; CAVALARO; GARCÍA, 2017) are backed by the real case study that presented problems during the lifting operation. Table 6 presents code recommendations available in design practice.

Table 6 - Code recommendations for lateral stability of concrete beams, (KRAHL; LIMA; EL

DEBS, 2015)

\begin{tabular}{|c|c|c|}
\hline \multirow{2}{*}{ Code } & \multicolumn{2}{|c|}{ Slenderness limit } \\
\hline & Permanent phase & Transitory phase \\
\hline Eurocode 2 & $\begin{array}{c}\ell_{0 \mathrm{f}} \mathrm{h}^{1 / 3} / \mathrm{b}_{\mathrm{f}}{ }^{4 / 3}<50 \\
\mathrm{~h} / \mathrm{b}_{\mathrm{f}}<2.5\end{array}$ & $\begin{array}{c}\ell_{\mathrm{Of}} \mathrm{h}^{1 / 3} / \mathrm{b}_{\mathrm{f}}^{4 / 3}<70 \\
\mathrm{~h} / \mathrm{b}_{\mathrm{f}}<3.5\end{array}$ \\
\hline ABNT NBR $9062^{2}$ & $\begin{array}{c}\ell_{0 \mathrm{f}} \mathrm{h} / \mathrm{b}_{\mathrm{f}}^{2}<500 \\
\ell_{0 \mathrm{f}} / \mathrm{b}_{\mathrm{f}}<50\end{array}$ & $\mathrm{~h}_{\mathrm{m}} / \mathrm{a}>2$ \\
\hline ACI $318-02^{1}$ & \multicolumn{2}{|c|}{$\ell_{0 \mathrm{f}} / \mathrm{b}_{\mathrm{f}}<50$} \\
\hline fib Model Code ${ }^{1}$ & \multicolumn{2}{|c|}{$\ell_{0 f} h^{1 / 3} / b_{f}{ }^{4 / 3}<50$} \\
\hline BS:8110-1 1 & \multicolumn{2}{|c|}{$\begin{array}{c}\ell_{0 \mathrm{f}} \mathrm{h} / \mathrm{b}_{\mathrm{f}}^{2}<250 \\
\ell_{\mathrm{Of}} / \mathrm{b}_{\mathrm{f}}<60\end{array}$} \\
\hline ABNT NBR $6118^{1}$ & \multicolumn{2}{|c|}{$\begin{array}{l}\mathrm{h} / \mathrm{b}_{\mathrm{f}}<2.5 \\
\ell_{0 \mathrm{f}} / \mathrm{b}_{\mathrm{f}}<50\end{array}$} \\
\hline EHE-08 & \multicolumn{2}{|c|}{$\delta_{0} \leq \ell / 750$} \\
\hline
\end{tabular}

$\ell_{\text {of: }}$ theoretical span or spacing between lateral restraints;

h: section height;

$b_{f}$ : compressed flange width. For rectangular section change $b_{f}$ for $b_{w}$;

$\mathrm{h}_{\mathrm{m}}$ : distance between the center of gravity and the support point;

a: elastic beam lateral displacement, considering the self-weight acting laterally;

$\delta_{0}$ : initial lateral displacement.

Notes ${ }^{1}$ do not distinguish between transitory and permanent phases ${ }^{2}$ As the current version do not contemplate the subject, it is being done concerning the previous version

\subsection{Reported experimental studies}

The recurrent register of accidents with beams during construction has pushed the researches developed studies to understand further the mechanical behavior of these large concrete elements subjected to transitory phases. However, the high cost to perform fullscale experiments in this field is reflected in the few reported ones. 


\section{Test to failure}

(SWANN; GODDEN, 1966) published the only test to failure of concrete beams lifted by cables. The elements were prestressed and the result reported was for a beam lifted at the ends with inclined cables by 44.8 degrees. The span of the element was $4.34 \mathrm{~m}$ and the height of $229 \mathrm{~mm}$. Hardly this beam would topple due to self-weight, then, to induce instability, the authors applied four equidistant concentrated loads over the span. Furthermore, a device was utilized to shift the attachment point of the lifting cable from the top flange. This measure can improve the capacity of the beam. Figure 29 (a) presents the test apparatus and the failed beam and Figure 29 (b) the load-rotation curve of the midspan and at lifting points.

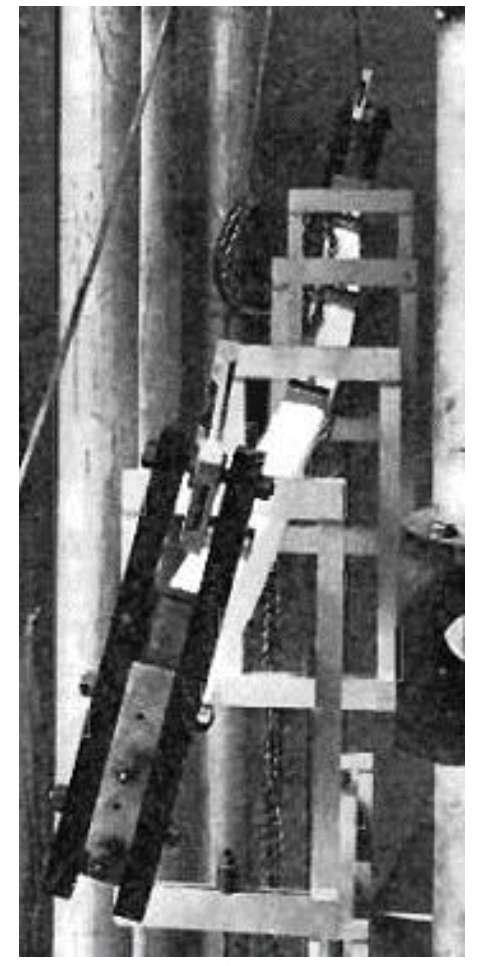

(a)

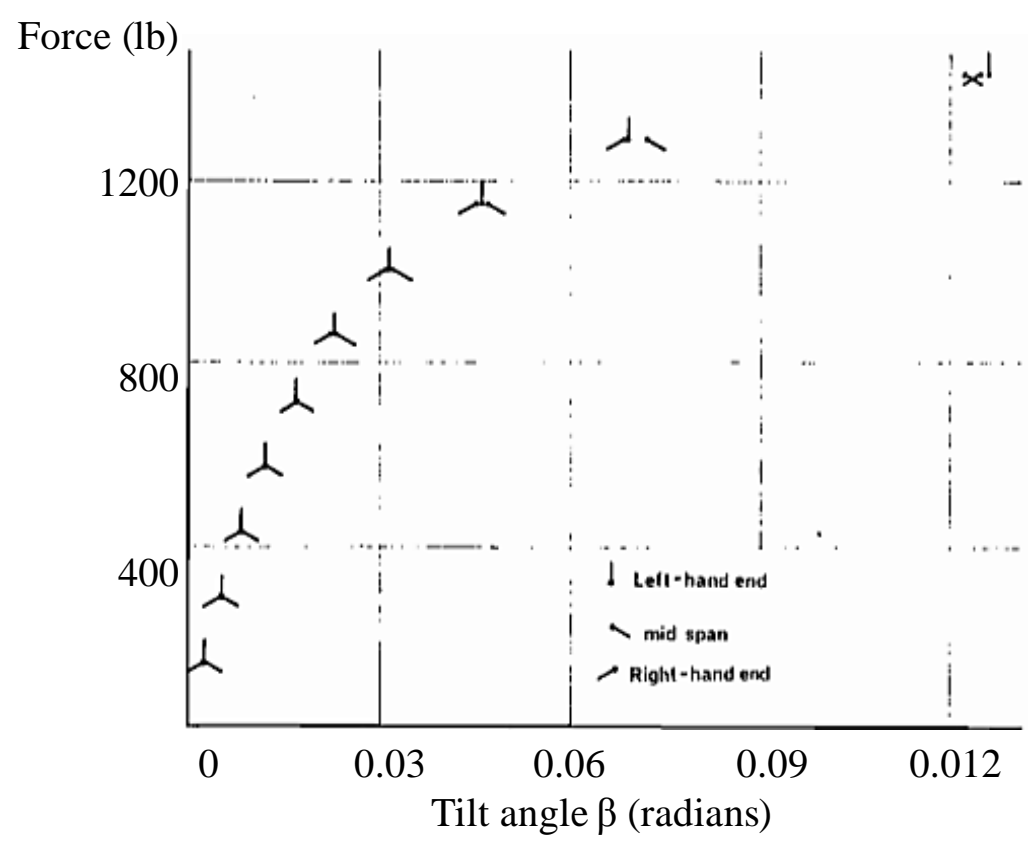

(b)

Figure 29 - (a) test apparatus and (b) total load-rotation curve for the test of (SWANN; GODDEN, 1966)

The nonlinear behavior is evident from the experimental curve. Also, the rotation was almost the same at the three points, which means that the beam undergoes preponderant rigid body rotation and lateral flexure. This evidence is reflected in the preponderant vertical flexural cracking on one side of the beam. Also, two further conclusions from the study were presented, warping strains were negligible, and no creep effect was detected during the test. 
Furthermore, the initial rotation was recorded for all beams that can be the effect of eccentricity of the lifting cables and initial lateral displacements which induces rotation.

(MAST, 1994) and (LIMA, 2002) presented results of tests to failure of precast prestressed and nonprestressed concrete beams, respectively, under controlled rotation imposed at both ends. These studies show the rotation capacity of concrete beams subjected only to self-weight. The beams have respectively $45.4 \mathrm{~m}$ and $6.5 \mathrm{~m}$. Although the tests do not constitute an instability test, they simulated closely what happens with the beams at transitory phases. Both studies showed that the beams undergo rigid body rotation with weak axis flexural strains. Figure 30 presents a view of the tests setup at high levels of rotation.

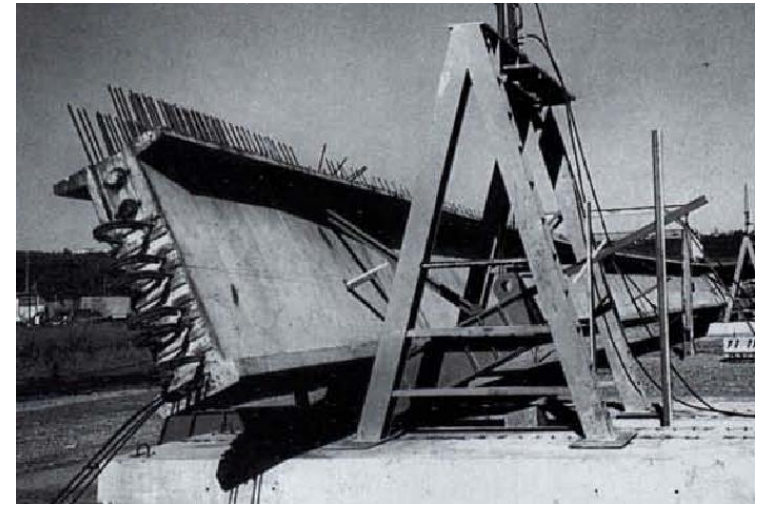

(a)

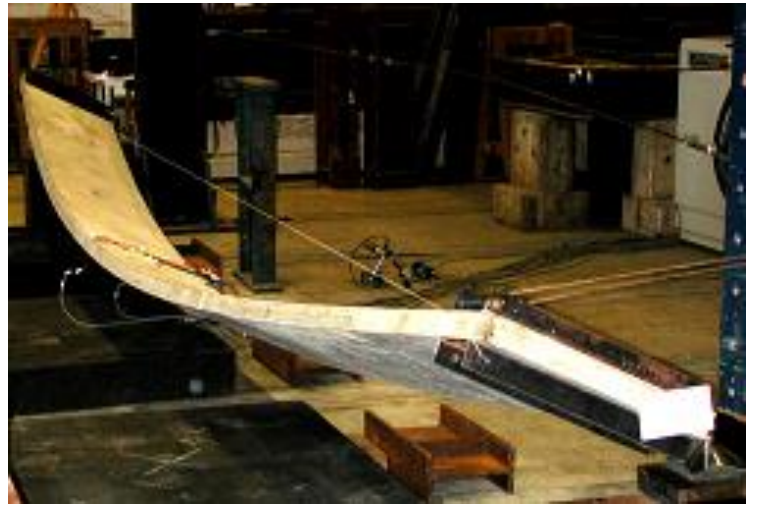

(b)

Figure 30 - Test performed by (a) (MAST, 1994) and (b) (LIMA, 2002)

These researches provided essential results about the behavior of concrete beam subjected to large displacements. The main results are the rotation capacity for cracking and failure. For the beam tested by (MAST, 1994), cracking was detected approximately at a rotation of 10 degrees, which corresponded to a lateral displacement of $127 \mathrm{~mm}$ and at failure a rotation of 32 degrees with $762 \mathrm{~mm}$ of lateral displacement at midspan. The cracking rotation for the beam tested by (LIMA, 2002) was 15 degrees and at failure 54 degrees. Figure 31 (a) and (b) presents the curves of rotation against lateral displacement of the mentioned studies, respectively. In Figure 31 (b), the legends Point 7, 8 and 9 represent the points at midspan that are respectively at the top, middle and bottom of the section. 


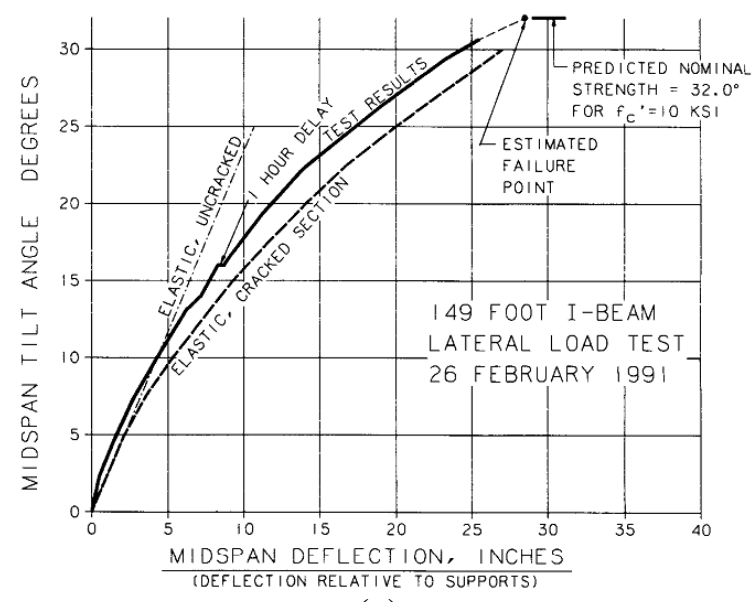

(a)

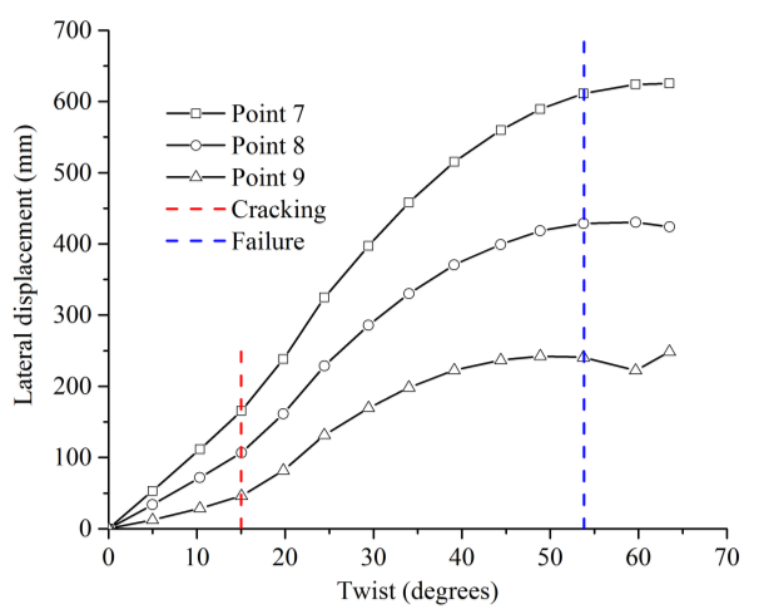

(b)

Figure 31 - Tests results of (a) (MAST, 1994) and (b) (LIMA, 2002)

Both studies showed that the elements suffer small torsional strains along the span when subjected only to self-weight and to a controlled rotation imposed at both ends. The consequence is that cracks are vertical due to the preponderant lateral flexure, Figure 32.

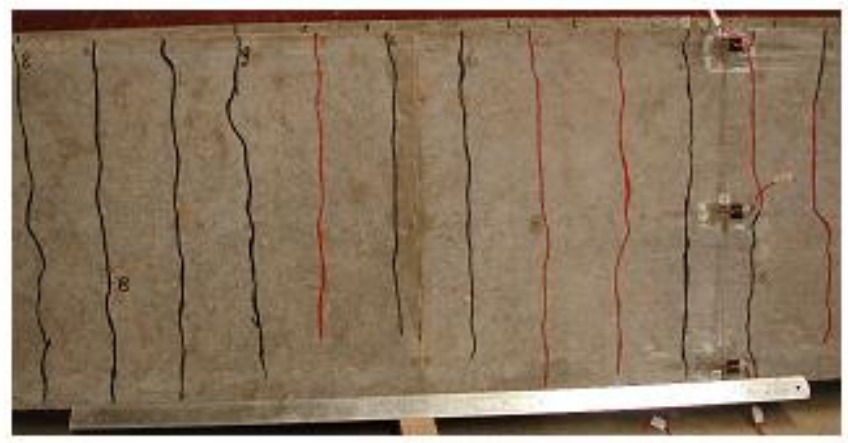

Figure 32 - Crack pattern of the beam tested by (LIMA, 2002)

Other important experimental test results were reported by (HURFF, 2010) and (CONSOLAZIO; HAMILTON, 2012). They tested prestressed beams supported exclusively by bearing pads. This situation constitutes a transitory phase very common during construction of precast structures. For large span bridges, steel reinforced elastomeric bearing pads are most common due to the higher loads. As discussed earlier, in this stage the rotation capacity of the pads and the planeness of the bottom face of the beam are fundamental for its stability. The beams can topple before cracking, that is, for small rotations due to the early loss of contact between beam and pads. (HURFF, 2010) tested the standard beam PCI BT-54 with a span of $30.5 \mathrm{~m}$ and (CONSOLAZIO; HAMILTON, 2012) designed an adapted cross-section from a standard beam with a reduced weak axis inertia with this same span. The first authors applied a concentrated load at midspan and the last 
applied symmetrical two-point loads. Figure 33 presents the test setup of (CONSOLAZIO; HAMILTON, 2012).

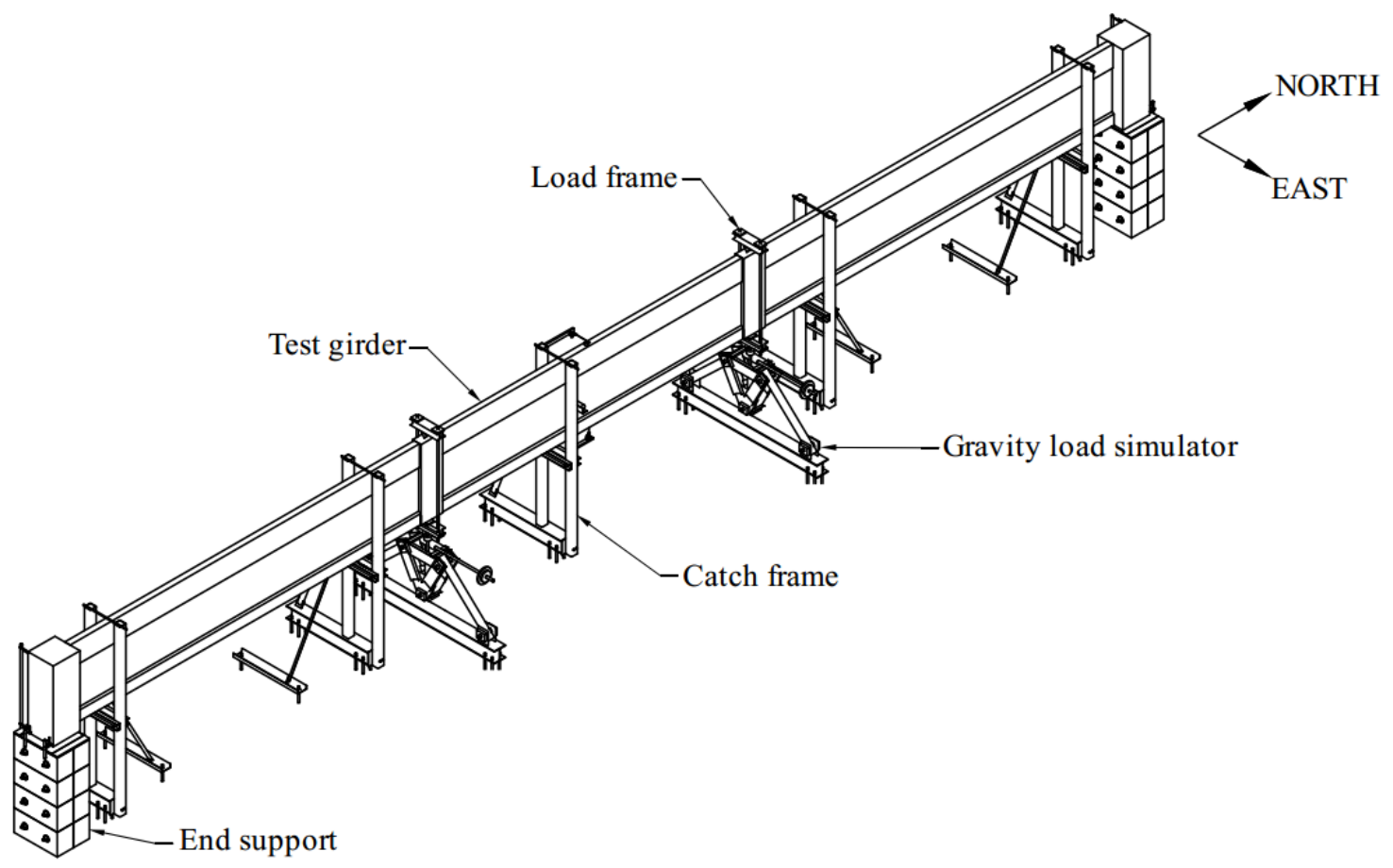

Figure 33 - Test setup for beam stability, (CONSOLAZIO; HAMILTON, 2012)

In both experimental programs, the loads were applied with a gravity load simulators. (CONSOLAZIO; HAMILTON, 2012) also investigated experimentally the stability of beams skewed or sloped relative to the support. Three beams were tested aligned and without slope (A-0-0), two beams were skewed by 45 degrees (A-45-0), three beams had a slope of $0.04 \mathrm{rad}$ (A-0-04), and one beam combined a skew angle of 15 degrees and a slope of 0.04 $\operatorname{rad}$ (A-15-04). Figure 34 (a) and (b) present the results of (HURFF, 2010) and (CONSOLAZIO; HAMILTON, 2012), respectively. The loading curve 1 in Figure 34 (a) corresponds to the first test performed with the BT 54, which presented an excessive early rotation. The test was stopped, and the bottom face of the beam was retrofitted. Then, the test carried again, and the result is the loading curve 2. 


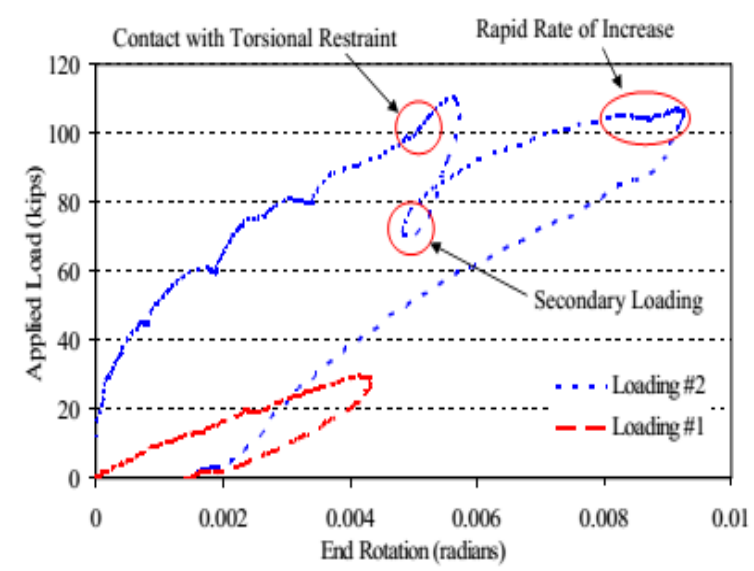

(a)

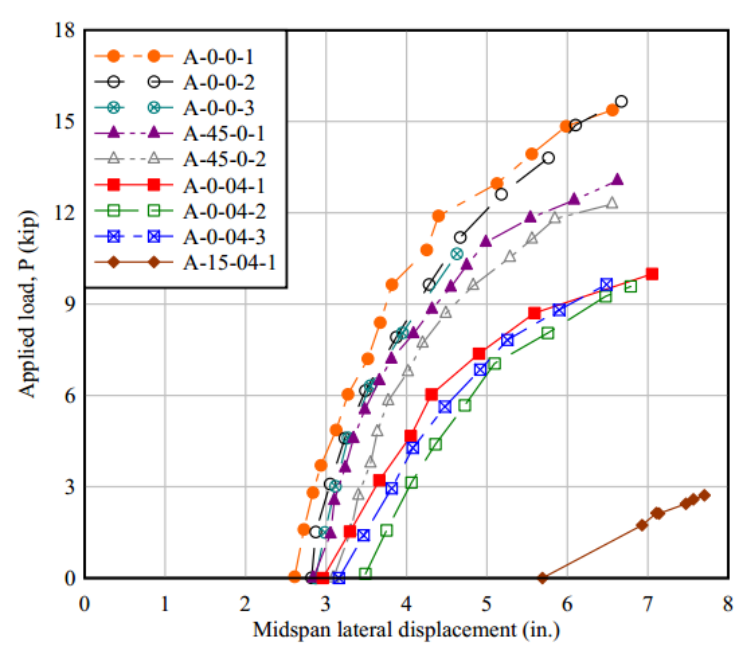

(b)

Figure 34 - Tests results of (a) (HURFF, 2010) and (b) (CONSOLAZIO; HAMILTON, 2012)

The effect of lack of flatness of the bottom face of was evident from the results on Figure 34 (a) comparing the two curves. Although it is expected that the bearing pads accommodate part of the misalignment of the concrete surface, it was not sufficient to maintain the load capacity of the beam. The decrease in capacity is mainly due to the reduction of the contact area, which consequently reduces the restraint against the toppling of the beam. This influence can be seen in the difference in the initial stiffness of the curves which is significantly smaller for the beam without retrofit. The loading curve 2 presented a typical instability behavior, that is, the load starts to increase at small rates and the rotation at a higher rate, which was observed after a rotation of 0.008 rad. Despite the unstable behavior, the beam did not crack. This hypothesis cannot be generalized. However, the beam reported by (HURFF, 2010) rotated almost 0.016 rad or 0.9 degrees at instability, and the cracking rotation of the beam tested by (MAST, 1994), which was 10 degrees. Thus, it is a plausible hypothesis that long beams undergo instability due to the nonlinear behavior of the pads. The results of (CONSOLAZIO; HAMILTON, 2012) also showed the influence of different bearing pad conditions. The beams with skewed pads A-45-0 presented a reduction of $16 \%$ in load capacity relative to the reference case (beams with aligned pads A-0-0). The beam with slopped supports presented a reduction of $23 \%$ to the reference case. The beams skewed and sloped to the pads had a large initial sweep, unacceptable in practice and purely overturned, according to the authors. This last case requires further investigations. Furthermore, the beams also did not crack.

(CONSOLAZIO; HAMILTON, 2012) also studied the behavior of the bearing pads experimentally under similar conditions to the transitory situation. The test procedure 
consisted in applying an axial load of the order of beam half self-weight and then a rotation to represent beam rollover. The pads were positioned to simulate aligned pad to the beam axis, skewed, sloped, and skewed and sloped simultaneously. This information is of great importance for analytical prediction of stability of beams, as the beams can topple when the pad is on nonlinear behavior, because mainly of the lift-off. Thus, the load capacity can be overestimated if the elastic stiffness of the bearing pads is utilized in formulations. (KRAHL; CARRAZEDO; EL DEBS, 2017) recommended the utilization of the secant stiffness of the moment-rotation curve to estimate the instability load.

Until today, the author does not identify any experimental test to failure/instability of beams supported by transport vehicles or supports that can simulate such conditions as superelevation of roads. (MAST, 1993) investigated the elastic rotational stiffness of the transport equipment but did not report about a possible nonlinear behavior of such components.

\section{Case studies}

Despite a significant number of accidents that are not scientifically investigated, see (KRAHL, 2014), and even reported, there are some papers and technical reports that care about the subject. The understanding of the possible causes of collapses can help significantly possible code recommendations and the development of safety measures to decrease the risks in such construction stages.

(OESTERLE et al., 2007) reported a detailed study about the collapse of nine concrete beams. The beams were placed on bearing pads and were left by three weeks without bracing before the collapse. Figure 35 presents the failed beams that had $34.7 \mathrm{~m}$ of span. 


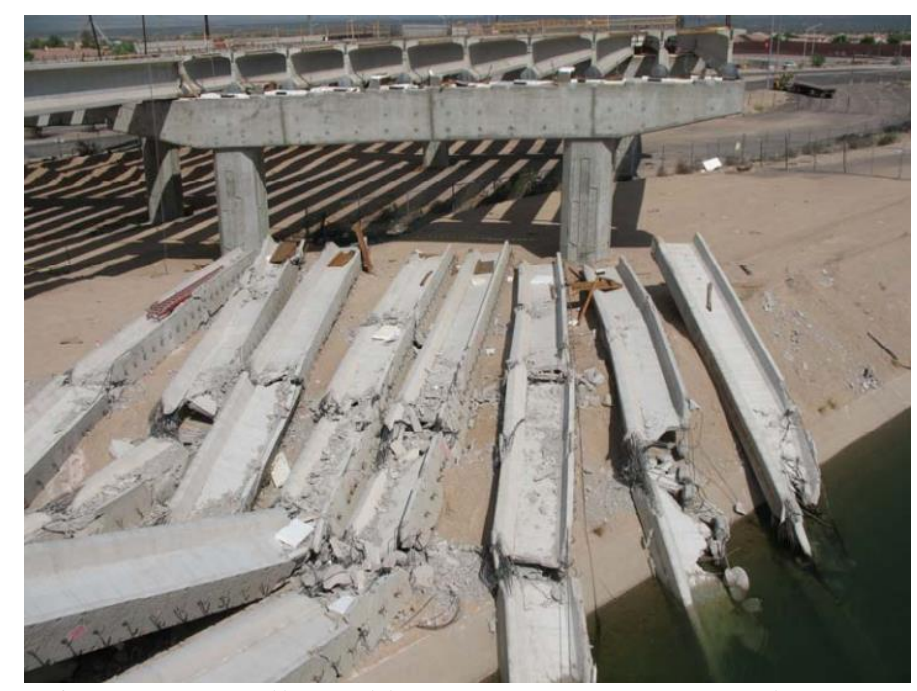

Figure 35 - Collapsed beams, (OESTERLE et al., 2007)

The authors concluded from an investigation that one beam toppled firstly and caused the progressive failure of the others by side impacts. The first beam instability was attributed to a critical combination of eccentricity of the bearing pad to the beam axis, initial imperfections including thermal and creep effects, and support slopes in longitudinal and transversal directions.

(BAIRÁN; CLADERA, 2014) investigated the partial collapse of a light roof sustained by precast beams with a span of $24.9 \mathrm{~m}$ supported by elastomeric bearing pads. The main cause of the accident was attributed to lack of rotational resistance provided by the bearing pads. The specified bearing pad had horizontal dimensions of $150 \mathrm{~mm}$ by $220 \mathrm{~mm}$. For a rectangular pad, the rotational stiffness is higher when the bigger dimension of the pad is positioned transversally to the beam axis. However, the pad was positioned with the smaller dimension in this direction. Thus, its stability capacity was considerably reduced due to this error. Combined with the decreased load bearing capacity, is a part of the construction of the roof one beam be eccentrically loaded when a light vault is placed, as presented in Figure 36, making the more critical.

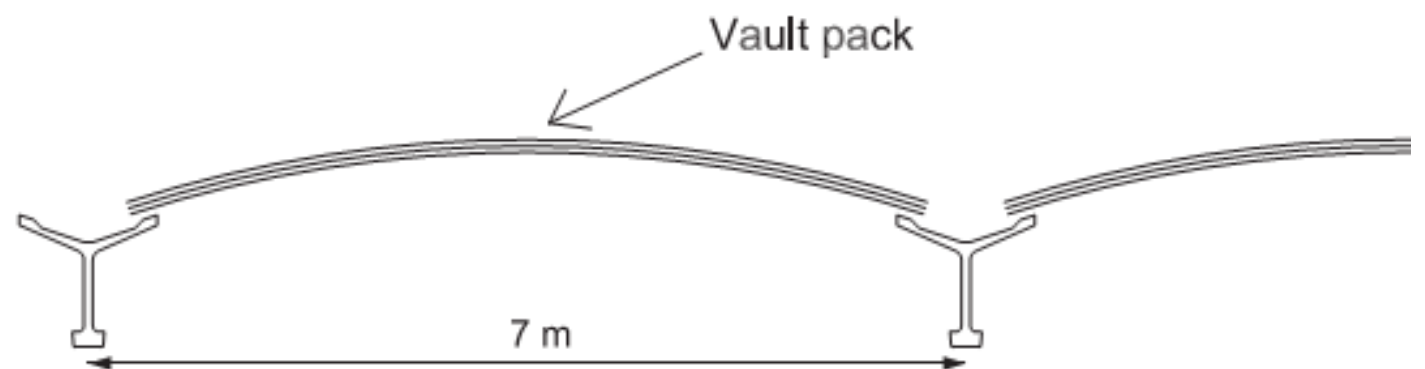

Axis 2

Axis 3

Figure 36 - Step of construction of the roof structure, (BAIRÁN; CLADERA, 2014) 
This combination may have caused the collapse of the beam at axis two. The authors discarded the influence of wind load based on an interview with the workers. However, no information was provided about solar radiation, initial imperfections, and further assembly errors that can influence on beam instability. However, this information is difficult to collect from a collapsed structure.

(DE LA FUENTE ANTEQUERA; CAVALARO; GARCÍA, 2017) reported a case study of a beam that presented problems during lifting. Two cranes lifted the beam trough vertical cables fixed $2 \mathrm{~m}$ from the ends. The beam was prestressed with a span of $45.6 \mathrm{~m}$. Figure 37 presents the beam that toppled.
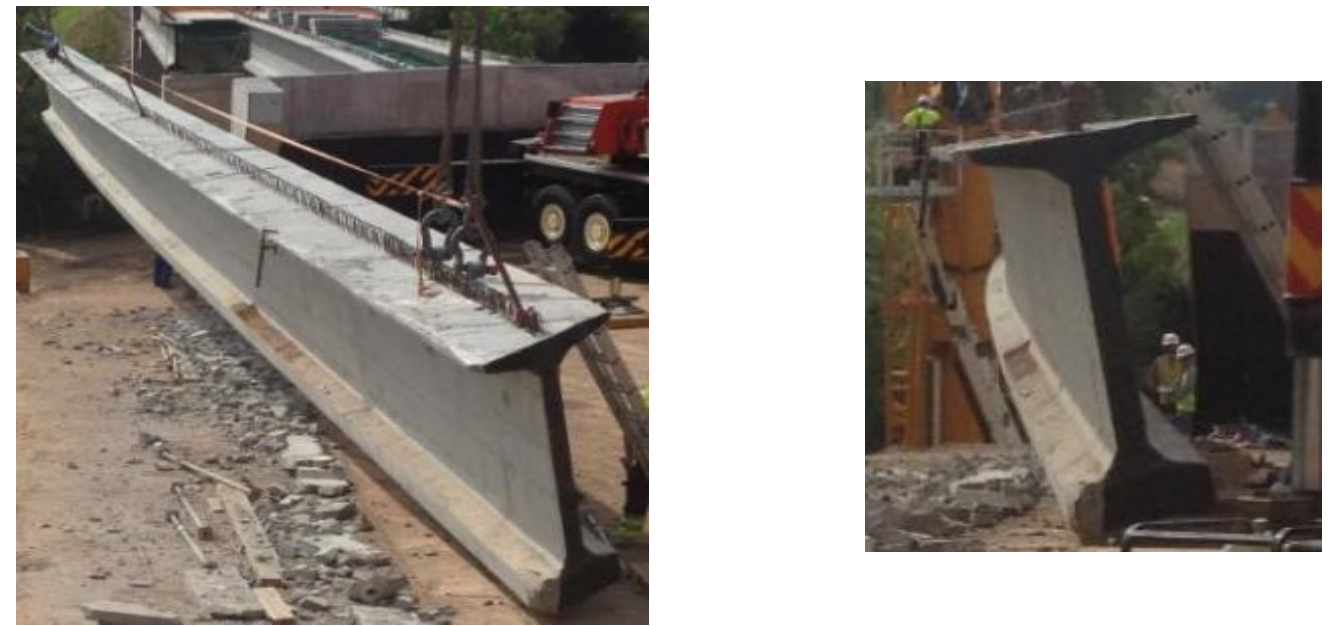

Figure 37 - Beam of the case study reported by (DE LA FUENTE ANTEQUERA; CAVALARO; GARCÍA, 2017)

The authors recorded eccentricity at attachment points of the lifting cables of $12 \mathrm{~mm}$, in addition to the measured lateral initial displacements of $90 \mathrm{~mm}$. At the beginning of the lifting process, the beam overturned and reached a lateral displacement of $300 \mathrm{~mm}$. Vertical cracks arisen at midspan and the beam had to be discarded.

\section{Monitoring tests}

(MCGORMLEY; LINDENBERG, 2016) developed an experimental study about beam behavior during the transitory phases of lifting and transport. The authors monitored two long prestressed concrete beams from the precast yard to the bridge site, with the purpose to verify the possible occurrence of damage in concrete. Dynamic strains and accelerations, thermal and rigid body movements were measured. The beams investigated were the standard BT-72 and LG-54, both with a span length of $39.6 \mathrm{~m}$. Figure 38 presents the 
positions where the measurements were made. The jeep tongue position is in section D and the midspan on section B.

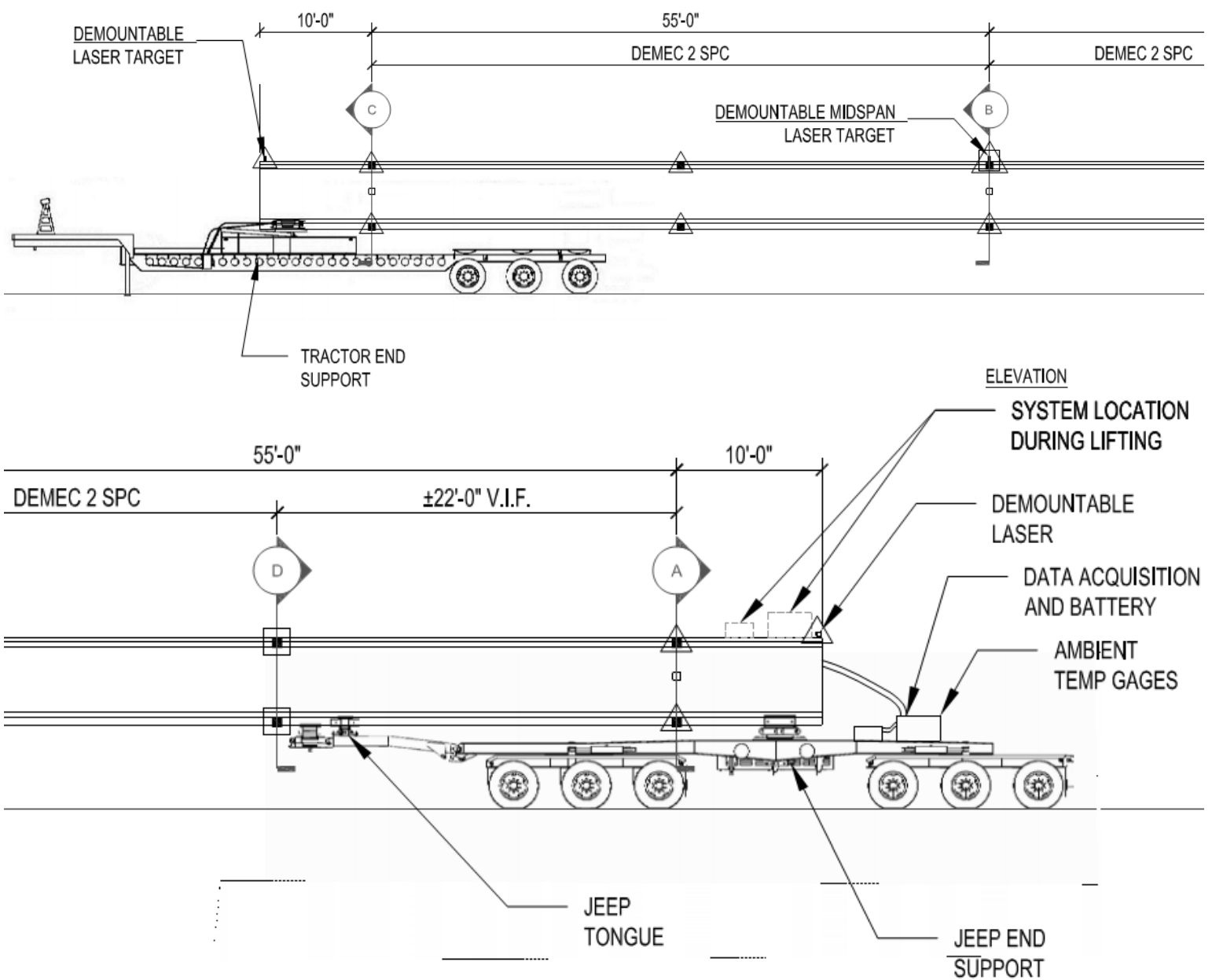

Figure 38 - Schematic representation of the beam BT-72 on a transport vehicle (divided into two draws), (MCGORMLEY; LINDENBERG, 2016)

(MCGORMLEY; LINDENBERG, 2016) estimated that cracking occurs between 132 and 200 microstrains for the concrete utilized in the beams. Figure 39 shows the strains measured at each of the four instrumented sections. 


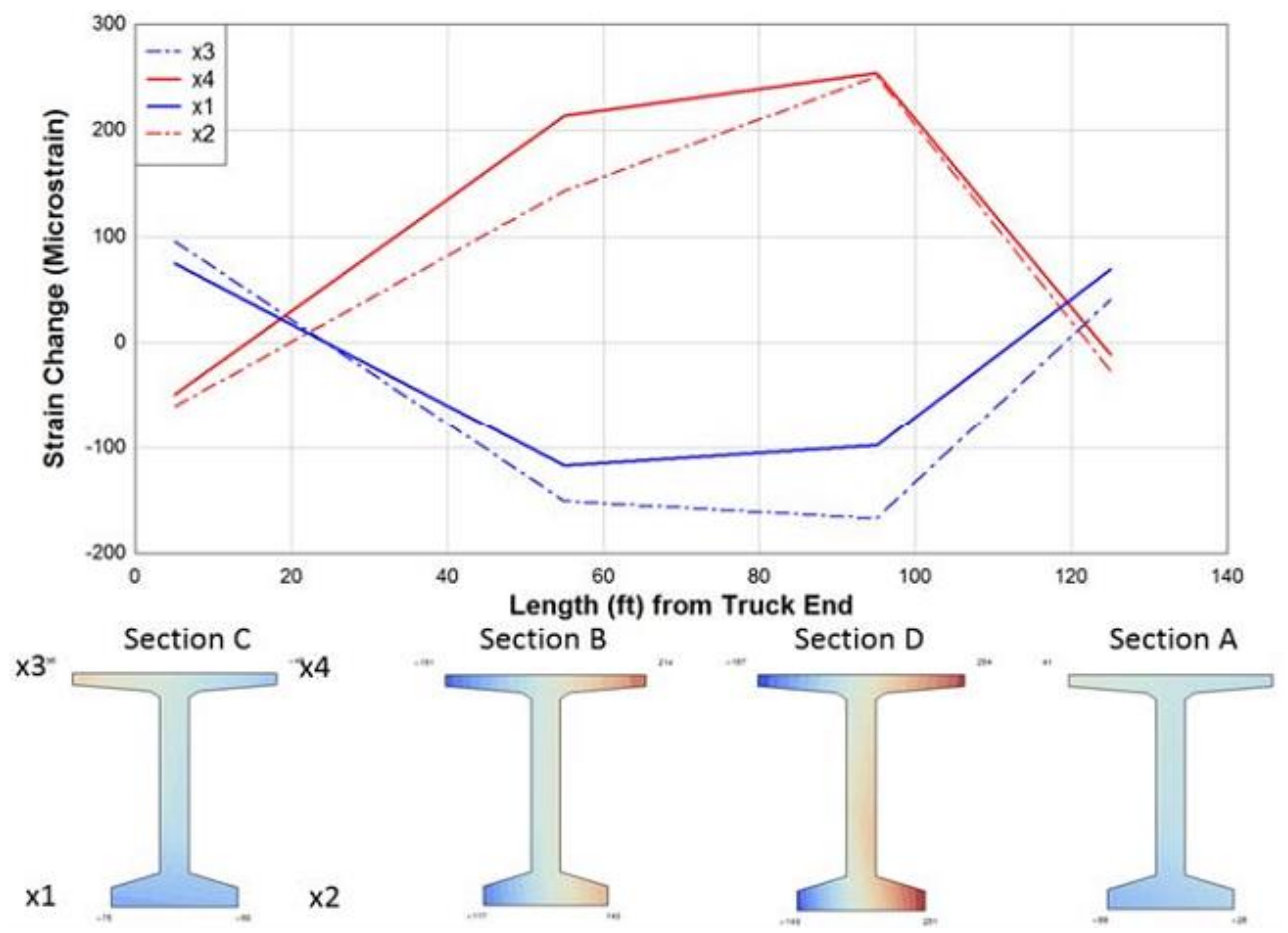

Figure 39 - Strains developed on the instrumented sections, (MCGORMLEY; LINDENBERG,

2016)

According to the investigation, the beams under transport presented more severe tensile strains when compared to lifting. From results in transport, the most critical section was at the jeep tongue (section D). However, the recorded strains in section B at midspan also exceeded the cracking strain.

The first hypothesis of the authors was that the possible cracking might be associated to high dynamic accelerations. However, the critical situations occurred at low-speed $(<16$ $\mathrm{km} / \mathrm{h}$ ) sharp turns. The find seems reasonable if we think that effect of inertial loads will act in a way to topple the beam out of the curve, as opposed to the effect of the self-weight that tends to topple the beam favorable to the superelevation of the road. Thus, in curves with low angles of superelevation, the inertial effects can be critical for high velocities. Otherwise, low velocities are critical when combined with high roll angles. However, more investigations are required to establish design guidelines.

Also, the authors investigated the possibility of cracking analytically. For this issue, they utilized the formulation proposed by (MAST, 1993). For the critical situation of low speed and high inclination of the road, the formulation did not predict any cracking. 


\subsection{Critical analysis of available analytical solutions}

The primary purpose of this topic is to present the central hypothesis of the current formulations available in literature and its correlation with the available experimental results of precast prestressed or non-prestressed beams. The first analytical proposition concerning the stability of concrete beams with flexible supports was published by (LEBELLE, 1959). The formulation constitutes an adaptation of the classical solutions of stability of beams, which accounts for lateral flexural stiffness $E I_{y}$ and torsional stiffness $G J$. The author introduced a parameter $\alpha$ that tries to penalize the critical load by considering the influence of the support flexibility for lifting, transport and when resting on bearing pads

$$
p_{\text {crit }}=16 k \sqrt{\alpha} \frac{\sqrt{E I_{y} G J}}{\ell^{3}}
$$

Where $k$ is a dimensionless parameter that account for further lateral restraint provided by the top and bottom flanges of the beam and $\ell$ is the span of the beam. Furthermore, as several uncertainties are involved during construction, at that time it was recommended a factor of safety of 4 for the critical load relative to the self-weight. However, this factor was arbitrary and require more investigations. Reliability-based methodologies can provide better recommendations.

As was discussed earlier, the beams undergo preponderantly rigid body rotation at transitory phases combined with lateral flexural strains. Thus, the function that represents rotation $\varphi(x)$ can be taken as a constant in these cases. Equation (22) represents the governing equations of lateral buckling.

$$
\begin{aligned}
& E I_{z} v^{\prime \prime}-M_{z}=0 \\
& E I_{y} u^{\prime \prime}-\varphi M_{z}=0 \\
& G J \varphi^{\prime}+E I_{w} \varphi^{\prime \prime}+u^{\prime} M_{z}=0
\end{aligned}
$$

Where, $v$ and $u$ represent the vertical and lateral displacements, respectively. $E I_{z}$ represents the major axis flexural stiffness, $E I_{y}$ represents the minor axis flexural stiffness, $G J$ Saint Venant torsional stiffness and warping stiffness $E I_{w}$. Due to the constant rotation assumed for transitory phases, the Saint Venant and warping torsional stiffness do not appear in the solution of the problem. The presence of this stiffness on the stability equation can overestimate the beam capacity, as $G J$ and $E I_{w}$ are directly proportional to the critical load $p_{\text {crit }}$, as in equation (21). Also, $M_{z}$ is the moment function due to the applied load. The first equation represents the flexure in the major axis. 


\section{Lifting}

For lifting phase, besides the proposition of (LEBELLE, 1959), (MULLER, 1962) proposed equations with a similar hypothesis, but now considering different loading conditions, as lateral distributed load and concentrated vertical loads. From these formulations, the increase in overhang length increases the load capacity of the beams significantly until a maximum and then decreases. However, the consideration of torsional stiffness and rotational support stiffness during lifting seems an unsafe hypothesis, which can predict higher values of critical load.

(ANDERSON, 1971) introduced a concept of safety factor in the problem of verification lateral stability of lifted beams. The idea is based on the assumption that during instability the beam only deflects laterally due to the much lesser weak axis moment of inertia compared to the strong axis moment of inertia. Besides, as the support does not restraint the beam to rotate no torsional strains occurs, then the beam rotate as a rigid body. The equilibrium was considered when the centroid at midspan and the points at top flange at the lifting points coincide vertically, see Figure 40.

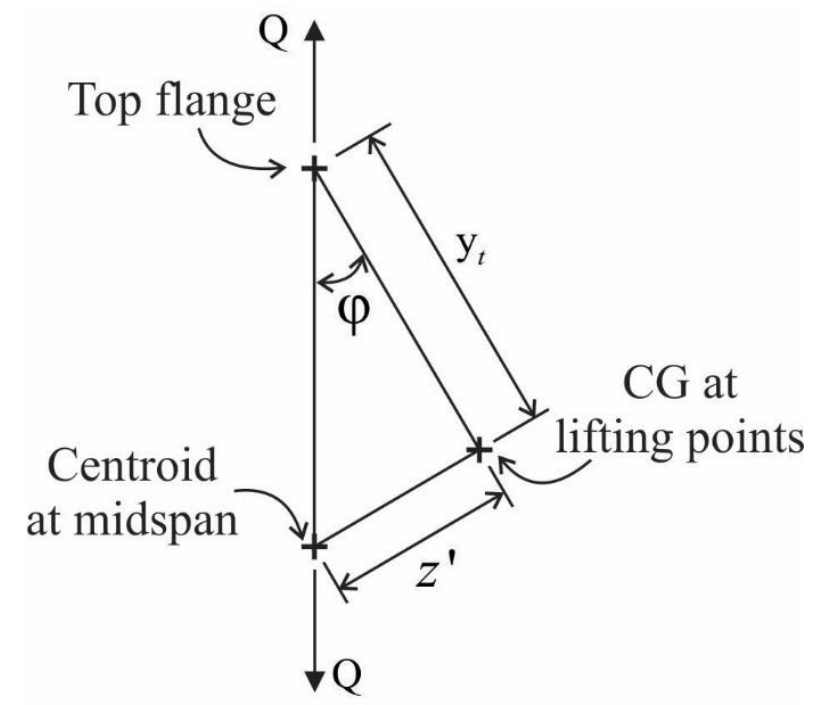

Figure 40 - Equilibrium condition for a lifted beam

Thus, the proposed factor of safety was established from the geometrical quantities of this figure. In the paper, (ANDERSON, 1971) refers to Mast the presented solution for beams lifted by cables. As is known, Mast considers the quantity $z^{\prime}$ equal $z^{\prime} 0 \sin \varphi$, where $z^{\prime} 0$ is the lateral elastic displacement of the beam subjected to the self-weight acting on the weak axis 


$$
\tan \varphi=\frac{z_{0}^{\prime} \sin \varphi}{y_{t}}
$$

As $y_{t}$ confers stability to the beam and the lateral displacement causes instability, the safety factor is

$$
F S=\frac{y_{t}}{z_{0}^{\prime}}
$$

This study was a forerunner of the main subsequent publications in the field, which adopted the same hypothesis and refined the analysis. (SWANN, 1971) proposed modifications for the equation (24) by recommending substitute $y_{t}$ by the distance between centroid and roll axis $y_{b}$ that not necessarily is on top flange, see Figure 27. Besides, instead of the total lateral elastic displacement of the beam centroid, the author recommended considered the lateral displacement of the center of mass. Also, essential observations and suggestions were made about the influence of initial imperfections and eccentric position of the lifting cables, which can reduce the factor of safety.

A consensus arisen from this researches was that the utilization of overhangs increases the stability capacity of prestressed beams. Also, (LASZLO; IMPER, 1987) highlighted that the increase in the concrete strength, the utilization of lateral bracing, as temporary trusses at the top flange, and rigid yokes, see Figure 41, also increase the beam capacity. Both can reduce the lateral displacement due to a possible roll caused by imperfections. Furthermore, the authors followed the hypothesis and recommendations of the previous two papers. Additionally, they recommended values of a factor of safety and addressed for verifications of allowable stresses at the time of the construction phases.

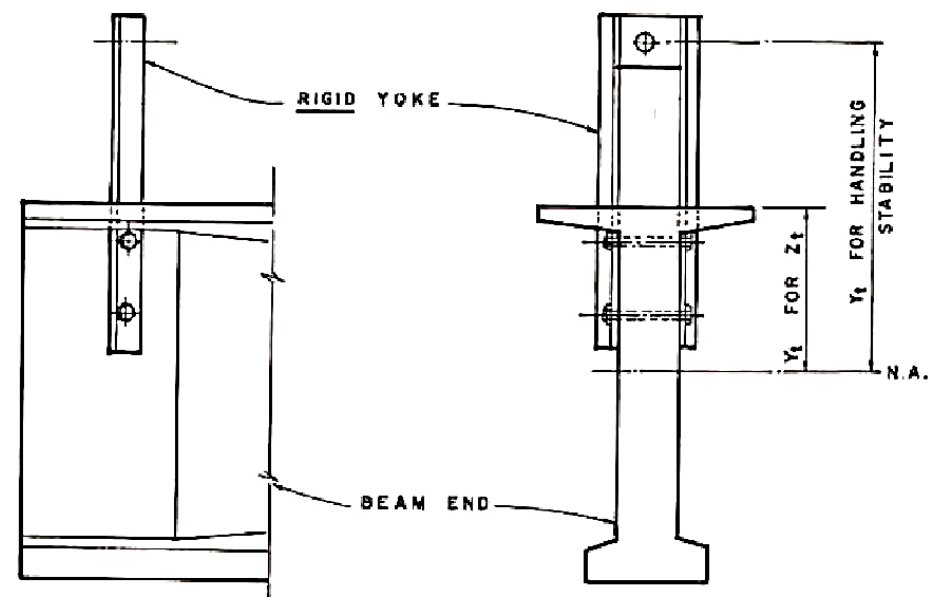

Figure 41 - Lifting device to increase beam stability capacity, (LASZLO; IMPER, 1987) 
(MAST, 1989) proposed an analytical formulation that considers the beam initially imperfect for stability checks. The central assumptions adopted in (ANDERSON, 1971) were also assumed in this formulation, which was called part 1 . The author redefined the equation (23) by considering at equilibrium an initially imperfect beam with initial lateral displacement $e_{i}$ and, as in previous studies, the support is rotation free that makes the beam initially rotated $\varphi_{i}$. The component of the total self-weight $Q \sin \varphi$ that arises acts on the weak axis causing an elastic lateral displacement $z$ ' and consequently a rotation $\varphi$. This problem is nonlinear because the calculation of $\varphi$ requires determining $z$ ' which depends on $\varphi$. The author affirms that the problem is solved by considering

$$
z^{\prime}=z_{0}^{\prime} \sin \varphi
$$

Where $z^{\prime} 0$ is the elastic displacement of the beam caused by the full weight $Q$ acting about the weak axis, which is determined from the theory of small displacements. Thus, (MAST, 1989) establishes the equilibrium equation

$$
\tan \varphi=\frac{\left(z_{0}^{\prime} \sin \varphi+e_{i}\right)}{y_{b}}
$$

This equation can be written, considering small displacements, as

$$
\left(\frac{y_{b}}{z_{0}^{\prime}}\right)_{\text {critical }}=\frac{1}{1-\frac{\varphi_{i}}{\varphi}}
$$

Thus, the factor of safety of an imperfect beam proposed by (MAST, 1989).

$$
F S=\frac{y_{b}}{z_{0}^{\prime}}\left(1-\frac{\varphi_{i}}{\varphi}\right)
$$

In part 2, which has been adopted by PCI Bridge Design Manual, (MAST, 1993) presented an alternative formulation based on the relation of moment arms of the moments caused by the self-weight and the reaction of attached cables relative to the centroid of the beam at the supports. This hypothesis results in a different factor of safety

$$
F S=\frac{y_{b} \sin \varphi}{\left(z^{\prime}+e_{i}\right) \cos \varphi}=\frac{1}{\frac{z_{0}^{\prime}}{y_{b}}+\frac{\varphi_{i}}{\varphi}}
$$

A geometrical interpretation can explain the differences between equations (24) and (25). Figure 42 represents the geometrical quantities considered in the formulation of (MAST, 1989) and (MAST, 1993), left and right figures respectively. 

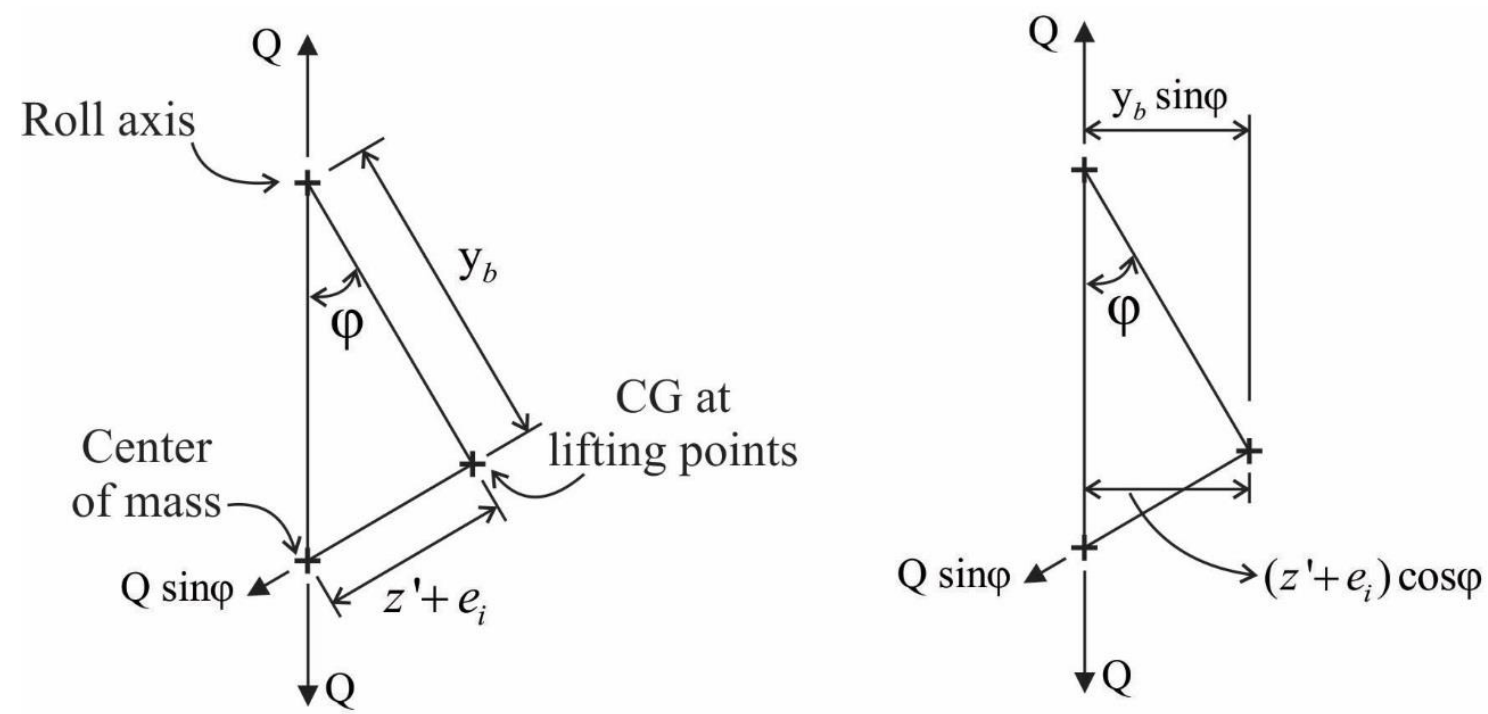

Figure 42 - Representation of the geometrical quantities utilized in the formulation of (MAST,

1993)

From the propositions of (MAST, 1989), there is some questionable hypothesis that must be pointed out, as the equilibrium condition shown in Figure 42. As long as lateral load exists $Q \sin \varphi$, the equilibrium is necessarily unstable, in contrast to what was considered. Furthermore, stability problems are geometric nonlinear and consequently the displacements are large. Thus, the proposed solution for $z^{\prime}$, equation (25), seems inconsistent, because it is a function of a theoretical displacement based on small displacement theory. Also, the approximations for the twist $(\sin \varphi \approx \tan \varphi \approx \varphi, \cos \varphi=1)$ at equilibrium are taken as small. Then, this formulation requires improvements to represent the behavior of long prestressed concrete beams.

(PEART; RHOMBERG; JAMES, 1992) investigated the influence of camber deflections due to prestress force in the stability of lifted beams. The solution for critical self-weight was determined based on the governing differential equations of the problem, including the effect of camber in the moment equations. The procedure was similar to the presented by (LEBELLE, 1959), then, the torsional twist was accounted. No closed-form solutions were presented, but the authors affirm, from the proposed formulation, that the camber effect can reduce more than $12 \%$ of the critical load.

(STRATFORD; BURGOYNE, 2000) presented a detailed study about concrete beams lifted by vertical and inclined cables. The initial imperfections were considered at equilibrium, as presented by (TIMOSHENKO; GERE, 1988). The solved differential equations of equilibrium are divided into two parts. The first governs the behavior between lifting points 


$$
M=-E I_{y}\left(v "-v_{0}\right)=V_{0}-\frac{\psi x^{2}}{2}+H v-M_{0}
$$

Moreover, at the overhangs

$$
M=-E I_{y}\left(v "-v_{0}\right)=-\frac{\psi(a+x)^{2}}{2}
$$

Where, $V_{0}$ is the minor-axis shear force between lifting points, $\psi$ is the lateral load parameter that accounts for self-weight and wind load, $H$ is the axial force due to the inclination of the cables, $M_{0}$ is the minor-axis moments acting between the lifting points, $v$, $v^{\prime \prime}$ are the lateral displacement function and the lateral curvature, respectively. Most of the cases studied required numerical solution. However, when the beam is lifted with vertical cables, (STRATFORD; BURGOYNE, 2000) presented a closed-form solution without considering the initial imperfection

$$
q_{c r}=\frac{120 y_{b} E I_{y}}{\ell^{4}\left(1-10 \frac{a}{\ell}+30 \frac{a^{2}}{\ell^{2}}-20 \frac{a^{3}}{\ell^{3}}-10 \frac{a^{4}}{\ell^{4}}\right)}
$$

Where $y_{b}$ is the height of the attachment points relative to the centroid, $E I_{y}$ is the lateral flexural stiffness, $\ell$ is the span of the beam, and $a$ is the overhang length. As seen, no torsional contributions appear. As an alternative, the equation proposed by (SOUTHWELL, 1932) enables to consider initial imperfection after calculating the instability load by equation (32). This topic is further discussed later.

(PLAUT; MOEN, 2012) developed a formulation for curved beams during lifting that can be reduced to almost straight beam problem when the considered curvature is small. From this hypothesis, the problem of lateral stability of imperfect beams can be solved, as the formulations account intrinsically for the effect of imperfections. Also, the equations enable to estimate the developed efforts and displacements during this construction stage.

\section{Beams on transport and on bearing pads}

Some of the formulations presented previously apply to verify the stability of beams on transport and on bearing pads by making some adaptations, as (LEBELLE, 1959) and (MAST, 1993). The situations are treated together because for both the axis of rotation is below the bottom flange of the beam, and the stiffness of the supports $k_{\theta}$ governs the stability. As discussed earlier, the mechanical of the problem is different from lifting because of $k_{\theta}$, 
and thus, at equilibrium, the center of mass of the beam can be displaced from the axis of rotation. The formulation proposed by (MAST, 1993) is

$$
F S=\frac{k_{\theta}(\varphi-\alpha)}{Q\left(z_{0}^{\prime} \varphi+y \varphi+e_{i}\right)}
$$

Where $Q$ is the total self-weight, $y$ is the distance between the centroid and the roll axis, $e_{i}$ is the initial lateral displacement, $\alpha$ is the superelevation angle, $z^{\prime}{ }_{0}$ is the lateral elastic displacement caused by $Q$ and $\varphi$ is the cracking or rupture angle. This formulation can be applied to both construction phases, but considering the changes in geometrical properties and $k_{\theta}$.

The critical buckling load for a perfect beam can be determined based on the solution of the equations of statics taken as the bifurcation of equilibrium, (TIMOSHENKO; GERE, 1988), an alternative way for energy method. (BURGOYNE; STRATFORD, 2001) developed a formulation based on this principle, which is different from what was done by (MAST, 1993). However, the same kinematic hypotheses were considered. Thus, for a littlerotated beam and from the equality between the rollover moment caused by the self-weight of the beam and the resisting moment $2 \varphi k_{\theta}$ provided by the supports

$$
w \ell\left(y_{b} \sin \varphi+z^{\prime} \cos \varphi\right)=2 \varphi k_{\theta}
$$

The terms between parentheses correspond to the lateral displacement considered as the applied moment arm, which is relative to the rotation permitted by the pads and the average lateral deflection due to the self-weight, respectively. (LEE, 2017) further developed the previous proposition by accounting the contribution of lateral displacements due to torsional strains

$$
w \ell\left[y_{b}\left(\varphi+\varphi_{t}\right)+z^{\prime}\right]=2 \varphi k_{\theta}
$$

$\varphi_{t}$ is the average torsional angle, which increases the applied moment arm and is determined by

$$
\begin{aligned}
& \varphi_{t}=\frac{2 \varphi_{t, \text { mid }}}{\pi} \\
& \varphi_{t, \text { mid }}=\left\{\begin{array}{l}
\left(2.4 e^{-0.1 \frac{h}{d_{w}}}\right) \varphi_{\text {crit }}, \quad \delta_{0} \leq \ell / 250 \\
\left(2.4 e^{-0.1 \frac{h}{d_{w}}}\right) \varphi_{\text {crit }}-\frac{3}{1000}\left(\delta_{0}-\frac{\ell}{250}\right) \varphi_{\text {crit }}, \quad \delta_{0}>\ell / 250
\end{array}\right.
\end{aligned}
$$


Where $\delta_{0}$ is the initial imperfection of the beam, $h$ is the height of the beam and $d_{w}$ is the width of the beam web.

(PLAUT; MOEN, 2014) proposed a formulation with the same hypothesis as for lifting, (PLAUT; MOEN, 2012), but, adapting the geometrical parameters. Furthermore, the authors adapted the formulation of (MAST, 1993) to consider wind load. Equation (25) changed to

$$
z^{\prime}=z_{0}^{\prime} \varepsilon
$$

Where $\varepsilon$ is

$$
\varepsilon=\sin \varphi+\eta \cos \varphi
$$

$\eta$ is a factor that relates the total wind load $W$ and self-weight. Thus, the adapted safety factor is

$$
F S=\frac{2 k_{\theta}(\varphi-\alpha)}{(1-\eta \varphi)\left[e_{i}+(\varphi+\eta) z_{0}^{\prime}\right]+(\varphi+\eta) y_{b}}
$$

Another possible way to consider the wind load is by increasing the lateral displacement $z^{\prime}{ }_{0}$ by summing the effect of wind, and then calculating the factor of safety as recommended by (MAST, 1993).

\section{Experimental measurement of parameter $k_{\theta}$}

As mentioned, the parameter $k_{\theta}$ governs the transitory phases when the beam is supported from below. (MAST, 1993) presented experimental results for truck and trailer rotational stiffness, which determined experimentally. The measurement consists of positioning the concrete beam eccentrically on the trailer and measure the linear relation between the moment caused by the beam self-weight and the rotation of the suspension system of the trailer, which results in $k_{\theta}$.

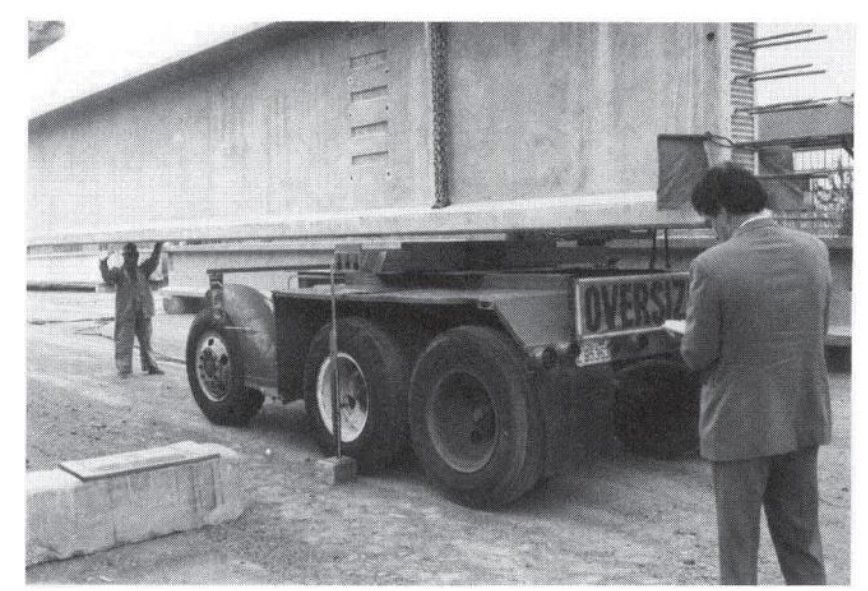

Figure 43 - Test to determine the truck roll stiffness, (MAST, 1993) 
From this study, (MAST, 1993) recommended that $k_{\theta}$ can be in the range of 340 to $680 \mathrm{kN} . \mathrm{m} / \mathrm{rad}$ per dual-tire axis. Then, the total value of $k_{\theta}$ to be considered is the sum of the contribution of truck and trailer. However, the author pointed out that this values are based on limited data and strongly recommends the calculation of actual roll stiffness of the transport vehicle for critical cases.

(CONSOLAZIO; HAMILTON, 2012) tested typical steel reinforced elastomeric bearing pads utilized in bridge construction. The test procedure consisted in loading the pads axially first, simulating the action of the self-weight when the beam is rested. Then, an incremental rotation is applied to simulate which happens when the beam rolls. For more details, please see the cited reference. Several conditions were investigated, as aligned (T-00 ) and skewed (T-45-0) pads. Besides, sloped aligned (T-0-02) and sloped skewed (T-4504) pads, for the case of simple supported beams with supports at different levels. Figure 44 presents the response of steel reinforced elastomeric bearing pads with horizontal dimensions of $355.6 \mathrm{~mm}$ per $609.6 \mathrm{~mm}$ and height of $65 \mathrm{~mm}$.

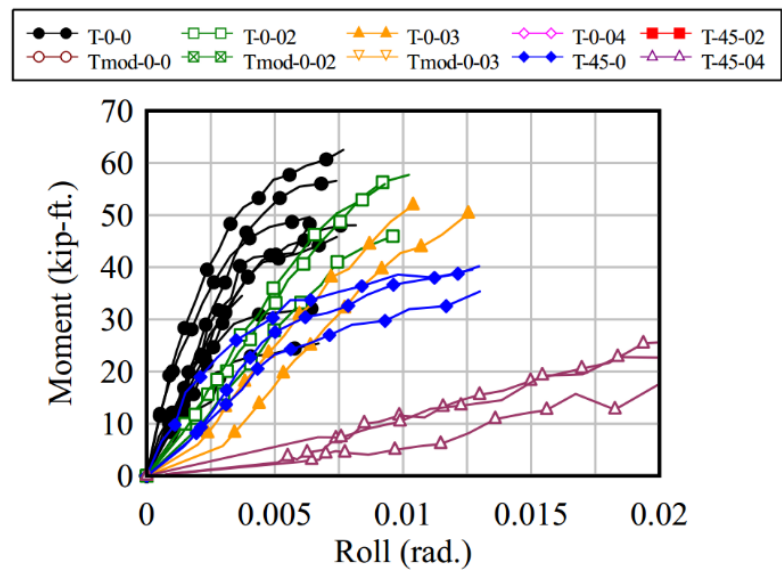

Figure 44 - Moment rotation response of the elastomeric bearing pad, (CONSOLAZIO;

HAMILTON, 2012)

As seen in the figure, for aligned and skewed pads without slope, the pads presented a nonlinear behavior after a certain level of the applied moment, which was more critical for the 45 degrees skewed pad. In the cases of the aligned pad with beam slope, the elastic rotational stiffness reduced with the increase in slope. However, as the test was stopped early, the nonlinear behavior was not detected but was expected. For the case which combines skew and slope, the reduction in stiffness was high. Then, the situation is critical as the elastic $k_{\theta}$ is very small compared to the other situations.

The rotation of the beam at supports during rollover can be higher than the rotation that limits the linear regime for $k_{\theta}$, due to the nonlinear behavior of the pads and lift-off 
effect. Thus, for analytical calculation, it is safer consider the secant value for rotational stiffness, which results in a smaller rollover load, (KRAHL; CARRAZEDO; EL DEBS, 2017). However, until now no analytical or empirical formulation stablish critical rotations to determine $k_{\theta}$.

\section{Account for initial imperfections}

The bifurcation solutions presented in the previous sections, equations (92), (93) and (95), represent the rollover instability load of perfect beams. The imperfections can be considered analytically in the problem of lateral buckling by the equations of (SOUTHWELL, 1932) and (MECK, 1977). However, (MANDAL; CALLADINE, 2002) showed that the Southwell's solution could predict the critical load for lateral buckling of beams, despite being developed to predict imperfect column buckling load. Also, (CHENG; YURA, 1988) compared the two equations to predicting their experimental results that presented almost the same critical loads for lateral buckling tests. Thus, the solution of Southwell is considered in the present research

$$
P_{\lim }=P_{c r} \sqrt[n]{1-\frac{\delta_{0}}{\delta_{\lim }}}
$$

Where $\delta_{\text {lim }}$ represents the displacement correspondent to the failure load $P_{\text {lim }}$. The index "lim" refers to limit point problem. $\delta_{0}$ is the initial sweep of the beam that must take into account effects like thermal load, shrinkage, manufacturing errors and others. $n$ is the exponent related to the nature of the instability problem which governs mainly the initial stiffness of the load-deflection curve. (LEE, 2017) recommended $n$ equal 1.5.

(CONSOLAZIO; HAMILTON, 2012) suggested a value for $\delta_{\text {lim }}$ at which the derivative of the nonlinear curve obtained through the Southwell equation represents onetenth of the initial value, see Figure 45. 


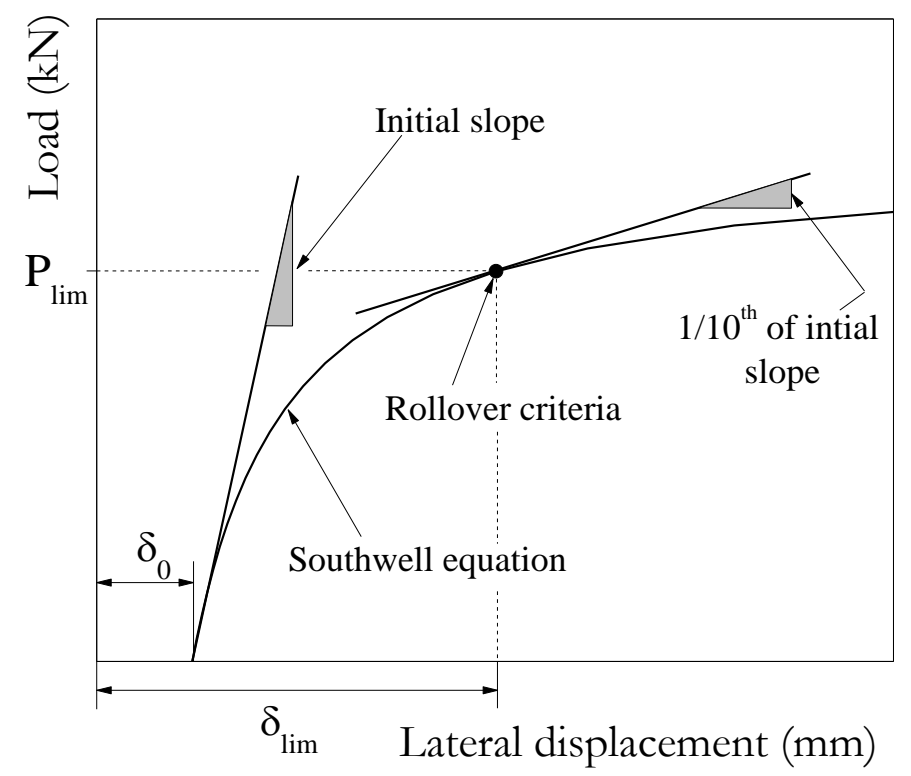

Figure 45 - Graphical representation of the rollover criteria

The slope of the load versus lateral deflection is the derivative of equation (40) considering $\delta_{\text {lim }}$ as the independent variable and $P_{l i m}$ as the dependent variable, equation (41).

$$
\frac{d\left(y=P_{\lim }\right)}{d\left(x=\delta_{\lim }\right)}=\frac{\delta_{0} P_{c r}\left(1-\frac{\delta_{0}}{\delta_{\lim }}\right)^{1 / n}}{n \delta_{\lim }^{2}\left(1-\frac{\delta_{0}}{\delta_{\lim }}\right)}
$$

The initial inclination occurs when $\delta_{0}=\delta_{\text {lim }}$. Thus, for $n=1$, equation (41) reduces to $P_{c r} \times \delta_{0} / \delta_{\text {lim }}^{2}$. For $n=2$, the derivative tends to infinite. Then, the initial inclination is vertical. The same occurs when $n=1.5$. As will be shown later, for the rollover of concrete beams, $n=1$ presents the better approximation for the initial stiffness of the experiments. Thus, the limit load occurs when equation (41) reaches one-tenth of $P_{c r} \times \delta_{0} / \delta_{\text {lim }}{ }^{2}$ when $n=1$, which corresponds to $\delta_{\text {lim }}=3.16 \times \delta_{0}$. This result corresponds to the adopted instability criteria.

Furthermore, lateral thermal gradients also increase the initial imperfections that took place during the fabrication process and must be accounted in the verification of the beam stability, (HURFF, 2010). This action represents an additional initial sweep and, therefore, the limit load is still predicted by the Southwell equation.

From tests with the PCI BT-72 beam with a span of $45.4 \mathrm{~m}$, (MAST, 1994) recorded a rotation capacity for cracking of approximately 10 degrees with the correspondent lateral displacement of $127 \mathrm{~mm}$ and for failure a rotation of 32 degrees with $762 \mathrm{~mm}$ of lateral displacement at midspan. Thus, for the present analyses, the $\delta_{\text {lim }}$ for cracking of beams will 
be adopted as the smaller between $3.16 \times \delta_{0}$ and $127 \mathrm{~mm}$. The smaller value results in a smaller limit load as the Southwell equation is asymptotic.

\subsection{Numerical studies}

\section{Concepts}

Numerical studies of concrete beams on transitory phases have been simulated through the framework of Finite Element Method (FEM). By utilizing FEM, one way to study consists in first investigate the elastic buckling loads and modes of the structure, (TRAHAIR, 1993). Then, utilize the buckling modes as the initial shape of the beams to simulate imperfections effects in the nonlinear geometric analysis. The first procedure is performed by solving the eigenvalue and eigenvector problem

$$
\left([K]-\lambda_{\text {crit }}[G]\right)\{\delta\}=\{0\}
$$

Where $[K]$ is the stiffness matrix, $[G]$ is the stability matrix, $\lambda_{c r i t}$ is the smaller real root of the determinant of the terms between parenthesis which corresponds to the eigenvalue and $\{\delta\}$ is the correspondent eigenvector.

The first eigenvectors can be amplified to represent initial imperfections. Thus, the simulation of a thin beam with such initial imperfection, and utilizing large displacements theory, results in lateral instability of the element. For the case where supports present torsional restraint, the beam develops flexural-torsional instability, and when the supports are flexible, the beam presents low torsional strains, and thus the problem corresponds to flexural instability with rigid body rotation.

The pre and post-buckling analyses consist of getting all load versus lateral displacement curve from a nonlinear geometrical analysis. The Newton-Raphson or Arclength algorithm can solve this kind of nonlinear problem.

A simple problem of lateral stability of beams was chosen to exemplify the procedure. The model was developed by the author of the thesis. A simple supported slender beam, free to warping, with a concentrated load at midspan is considered. Initially, the applied load has a unity value such as the first eigenvalue corresponds to the buckling load. The first buckling mode is presented in Figure 46, which corresponds to the beam laterally displaced that occurs due to the smaller lateral flexural stiffness compared to the major axis 
stiffness. The legend at the figure corresponds to the scale of lateral displacements. As can be seen, the maximum value is 1 .

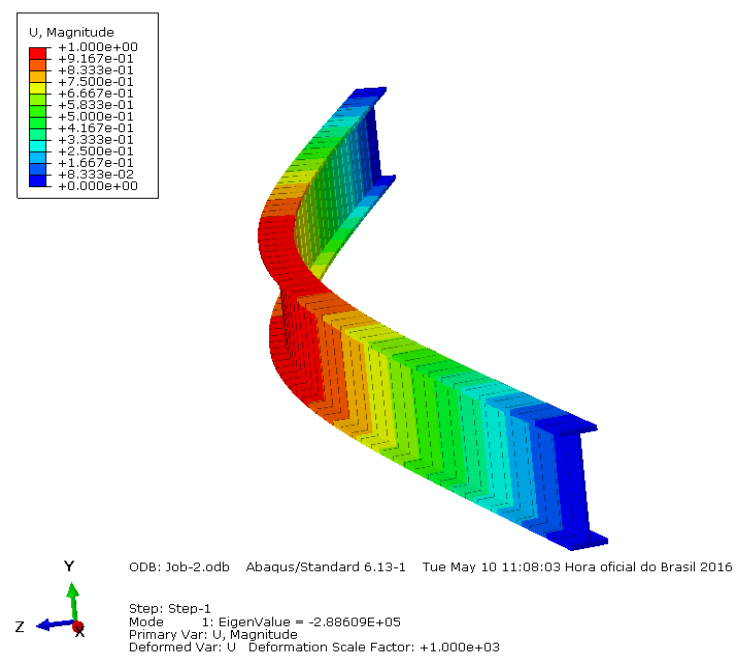

Figure 46 - First buckling mode of the beam

Then, with the first buckling mode imported, the geometric nonlinear analysis is performed. The shape of the beam is amplified for a chosen maximum initial imperfection at midspan. The boundary conditions are the same, but now the applied concentrated load is of the magnitude of the predicted elastic buckling load and applied in increments to evaluate the effect of large displacements, for an initially displaced beam. The curves of load versus lateral displacement are presented in Figure 47 together with the elastic buckling load in dashed line. Three different values of initial imperfection were assumed.

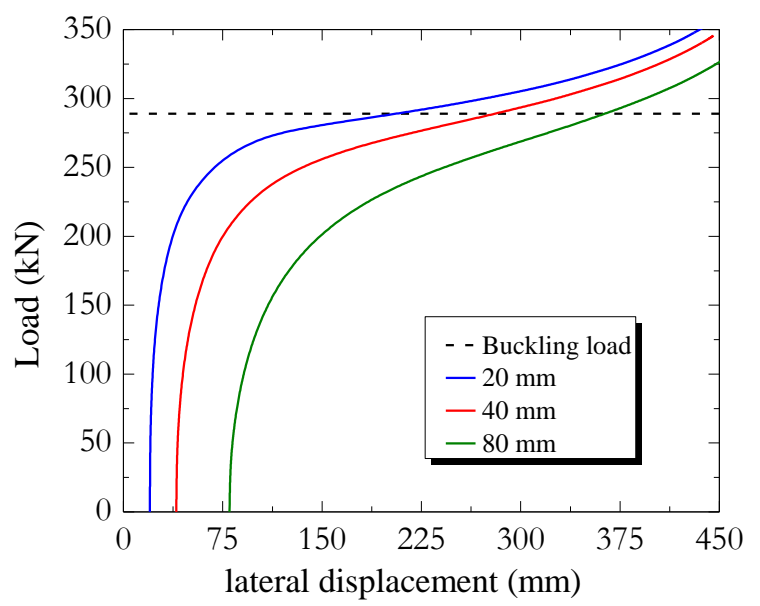

Figure 47 - Buckling load and load versus lateral displacement curves

It can be seen from Figure 47 that as the load increases to the level of buckling load the rate in increasing of lateral displacement becomes large while the rate of increase in load becomes smaller. Besides, the effects of the increase in lateral initial imperfections of the beam are shown. As observed, for the smaller initial imperfection, the nonlinear effects start 
to manifest near the buckling load. However, for the higher initial imperfection, the nonlinear effects manifest earlier. This behavior means that the large the initial displacement, the earlier the nonlinear effects.

To further illustrate the implication of the earlier geometrical nonlinear effects, the nonlinear material behavior is considered in the problem by imposing a limit Von Mises stress that causes plasticizing of the material. Figure 48 presents, in dashed and dot lines, the curves correspondent to the nonlinear analysis considering material and geometrical nonlinear behaviors together. The geometrical nonlinear results are presented again.

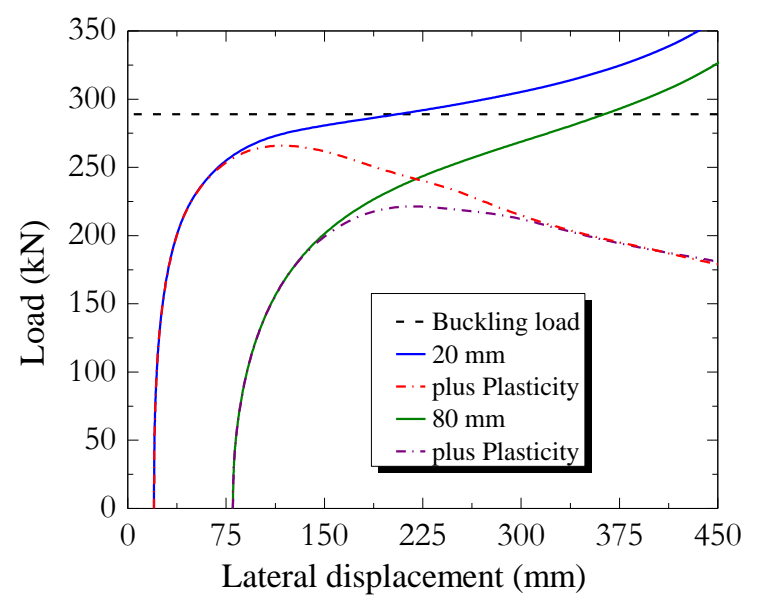

Figure 48 - Load versus lateral displacement response of the I-beam considering the nonlinear material behavior

Figure 48 shows that the nonlinear material behavior makes the strength of the beam smaller than the elastic buckling load. The peak load in these cases is commonly called limit load. Besides, for the beam with higher imperfection, as the geometrical nonlinear effects manifest earlier (torsional and lateral flexural moments), the limit load is lesser compared to the beam with smaller imperfection.

This methodology to study pre-buckling, buckling and post-buckling behavior of beams is general, (TRAHAIR, 1993). The major difference for transitory phases are the boundary conditions. Furthermore, initial imperfections can be inputted in other ways, as constructed the imperfect beam mesh manually since the shape of imperfections be known previously.

\section{Published research}

In the context of numerical simulation of transitory phases of precast beams with FEM, the study of (LIMA, 2002) was a precursor. The focus on simulation was in material 
behavior during imposed large rotations, as in the experiments of (MAST, 1994) and the presented in Lima's thesis. The constitutive model implemented considers the nonlinear behavior of concrete in compression and tension-stiffening for a specified region of influence rounding the reinforcement, and also the reduction of torsional stiffness. Furthermore, the numerical model can simulate prestressed concrete beams. Several examples are presented in (LIMA, 2002). Figure 49 (a) presents the comparisons between numerical and experimental curves and Figure 49 (b) the evolution of cracking in simulation as the beam was rotated.

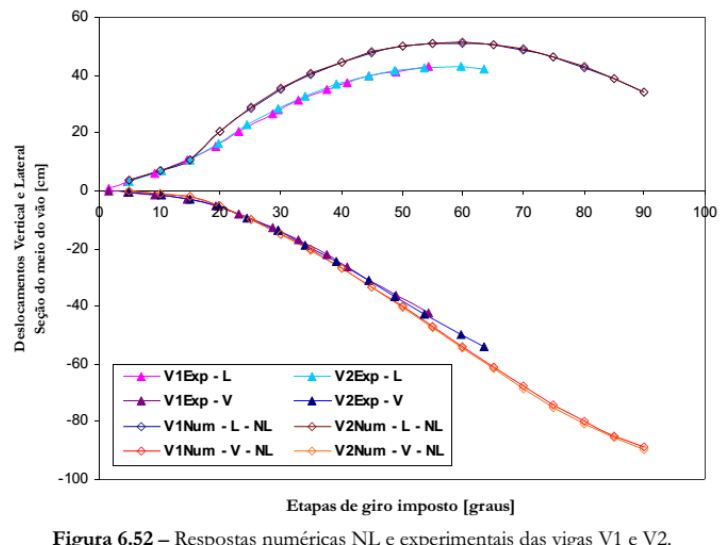

(a)

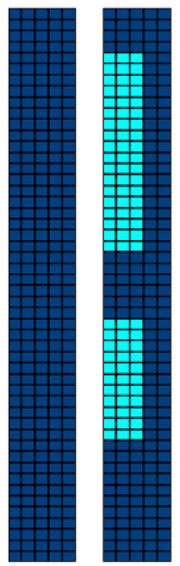

$\begin{array}{lll}\text { (a) } 0 \text { a } 15^{\circ} & \text { (b) } 20^{\circ}\end{array}$

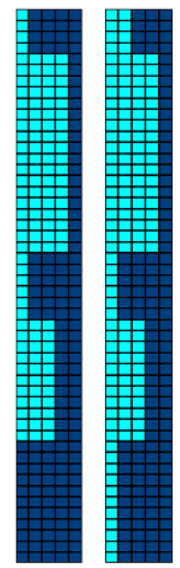

(c) $25^{\circ}$

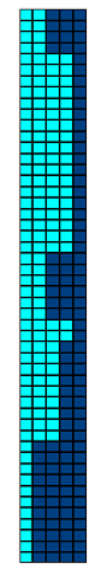

(b)

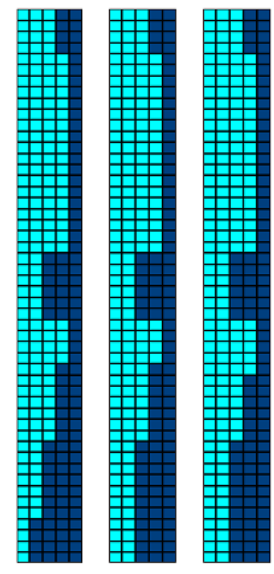

$\begin{array}{lll}\text { (f) } 40^{\circ} & \text { (g) } 45^{\circ} & \text { (h) } 50^{\circ}\end{array}$

Figure 49 - Experimental and numerical results of (LIMA, 2002)

The developed numerical model was capable of predicting with accuracy the nonlinear behavior of concrete regarding first cracking and variation of stiffness. Besides, the model was capable of detecting steel yielding and cracking evolution in the midspan cross section.

(COJOCARU, 2012) studied the lifting problem with vertical cables numerically. The author studied the beams Type IV of AASHTO and PCI BT-72, with spans of $31.7 \mathrm{~m}$, overhangs of $0.8 \mathrm{~m}$ and initial imperfection of $\ell / 960$, where $\ell$ is the span length. Two-node beam type finite elements were used for the concrete beam, which had self-weigh applied in the shear center. The cables were modeled with truss type finite elements, which were connected to the beam from the attachment points by rigid beam links. The model restrictions were applied at the top of the cables. Figure 50 presents the model. 


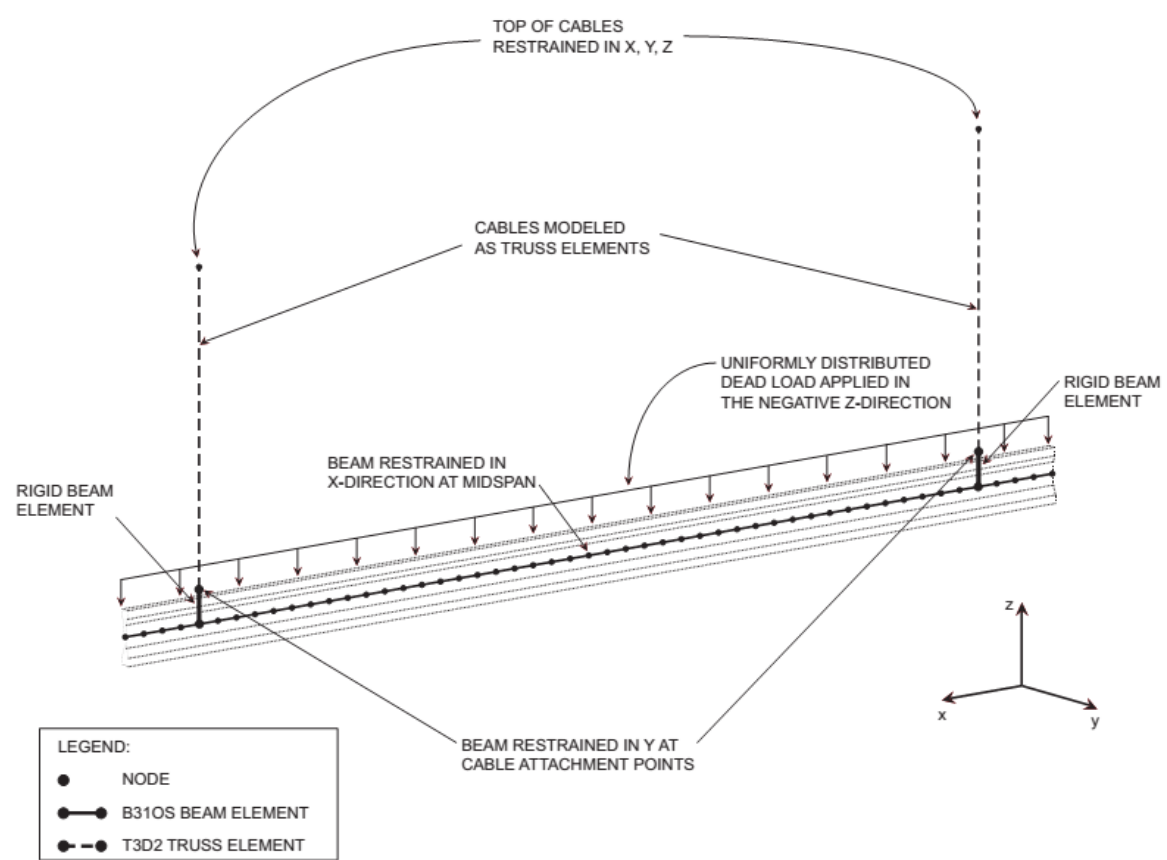

Figure 50 - Finite element model for beam lifted by cables, (COJOCARU, 2012)

(COJOCARU, 2012) compared the results of the proposed methodology with the analytical formulation developed by (PLAUT; MOEN, 2012). Figure 51 (a) and (b) presents the lateral displacements respectively at equilibrium of the beams Type IV of AASHTO and PCI BT-72 supporting self-weight. Figure 52 (a) and (b) presents the comparisons of the lateral bending moment by the two methodologies respectively.

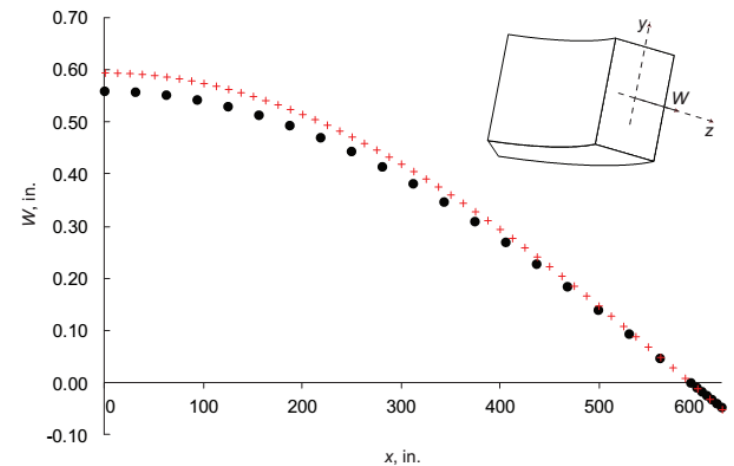

(a)

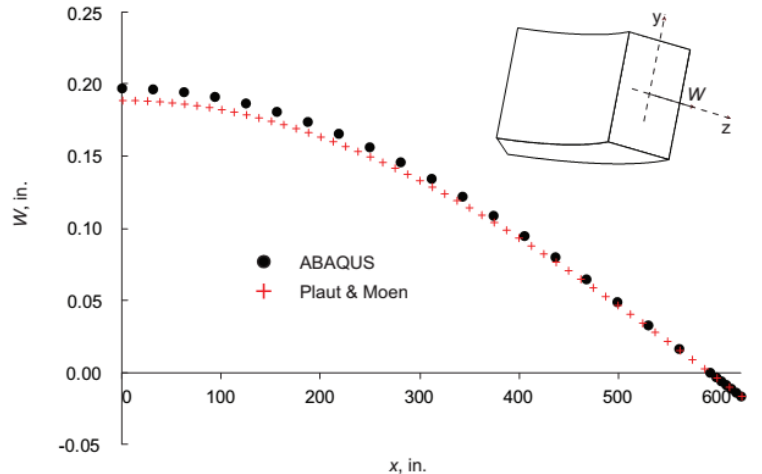

(b)

Figure 51 - Weak-axis displacement comparison for (a) Type IV and (b) BT-72, (COJOCARU, 2012) 

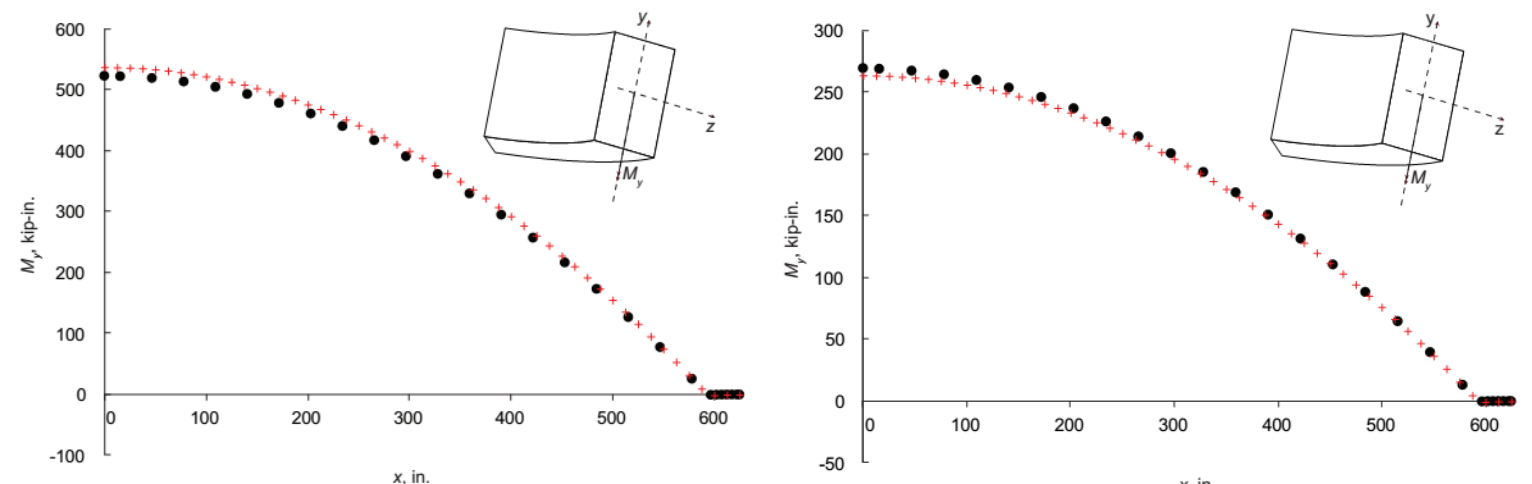

Figure 52 - Weak-axis bending moment comparison for (a) Type IV and (b) BT-72, (COJOCARU,

The proposed model by (COJOCARU, 2012) presented good correlation with the analytical formulation of (PLAUT; MOEN, 2012). Both, displacements and bending moment were predicted. It should be pointed out that the model does not consider nonlinear material behavior.

(CONSOLAZIO; HAMILTON, 2012) proposed a similar model to that of (COJOCARU, 2012) to simulate beams resting on bearing pads. The concrete beam was modeled with beam type finite elements. As external third-point loads were applied in the experimental model at the beam top face, rigid beam links were created to account for the effect of load height. Also, initial imperfections were considered in the analysis. The main difference for the previous model was in the modeling of bearing pads that govern the stability. The authors utilized a set of six translational and rotational springs. All springs were linear, excepting for the rotational spring relative to the longitudinal axis of the beams. The nonlinear spring was modeled to account for the pad nonlinear behavior, as observed experimentally. The pads were attached to the beam also by a rigid beam link with length correspondent to the distance between centroid and center of the bearing pad, see Figure 53.

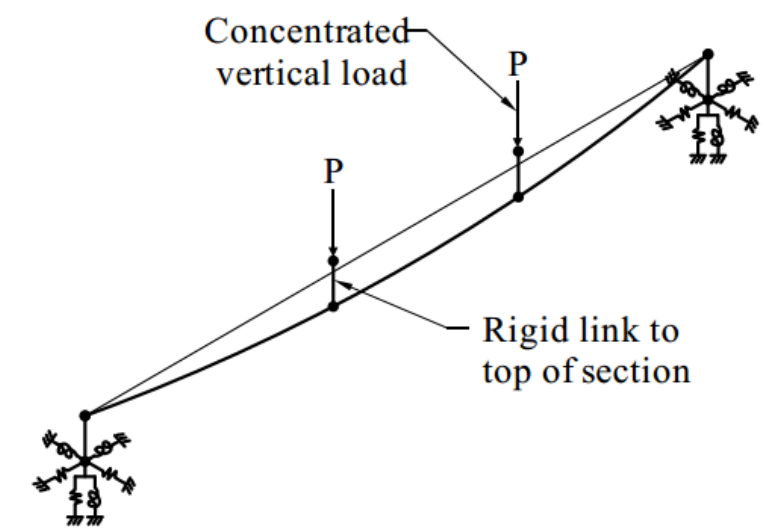

Figure 53 - Scheme of the finite element model, (CONSOLAZIO; HAMILTON, 2012) 
The proposed numerical model was compared to the experimental results obtained in the same research. The failure criteria adopted for analysis was for the step of nonlinear analysis that the stiffness of the beam reaches one-tenth of its initial value. Figure 54 presents the comparison between numerical and experimental results of (CONSOLAZIO; HAMILTON, 2012).

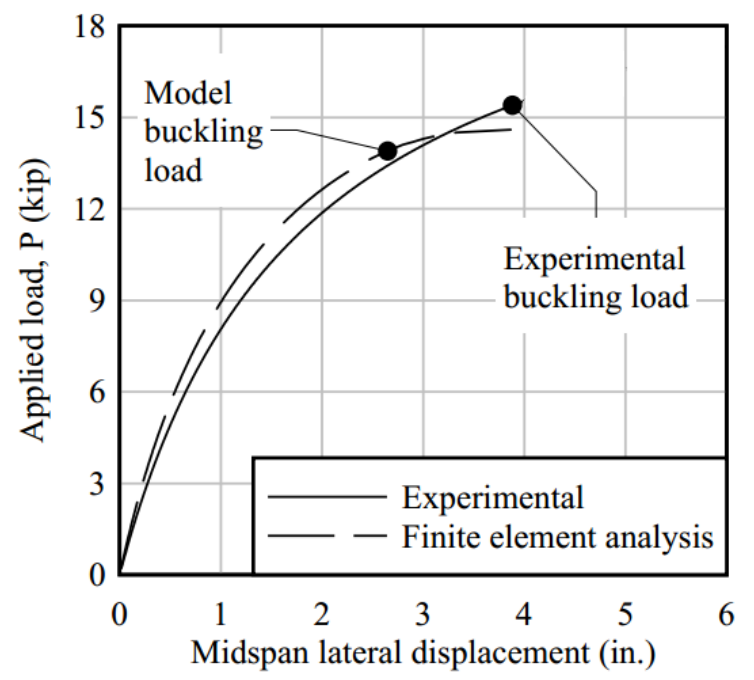

Figure 54 - Numerical and experimental results of (CONSOLAZIO; HAMILTON, 2012)

The difference between numerical and experimental critical loads was $9.7 \%$. Besides, all the load-displacement curve were predicted with accuracy. The author also simulated the tested beam with pads skewed 45 degrees and sloped $0.04 \mathrm{rad}$. The differences in numerical and experimental critical loads were $14.9 \%$ and $3.4 \%$, respectively. Although the tested beam was prestressed, the numerical model without prestressing predicted well the experimental behavior. Furthermore, material nonlinear behavior was not considered.

(LEE et al., 2017b) simulated the problem considering the pad as a grillage with nonlinear translational springs attached at nodes. The springs do not support tensile load to simulate the lift-off. Under compression, the axial nonlinear behavior of the pad is attributed to each spring correspondent to its tributary area. Figure 55 presents the pad finite element model. 


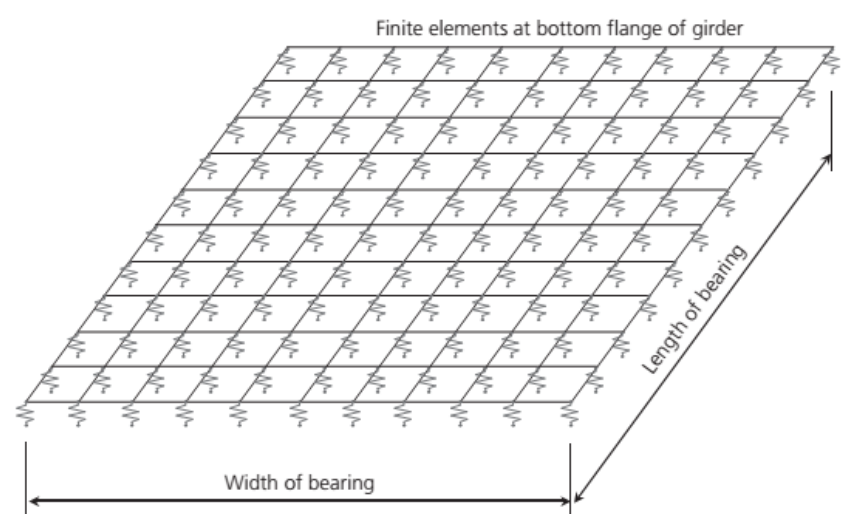

Figure 55 - Bearing pad model of (LEE et al., 2017b)

The authors investigated the influence of the type of finite element adopted in the stability problem. The elements were solid, shell and beam. Figure 56 presents the results.

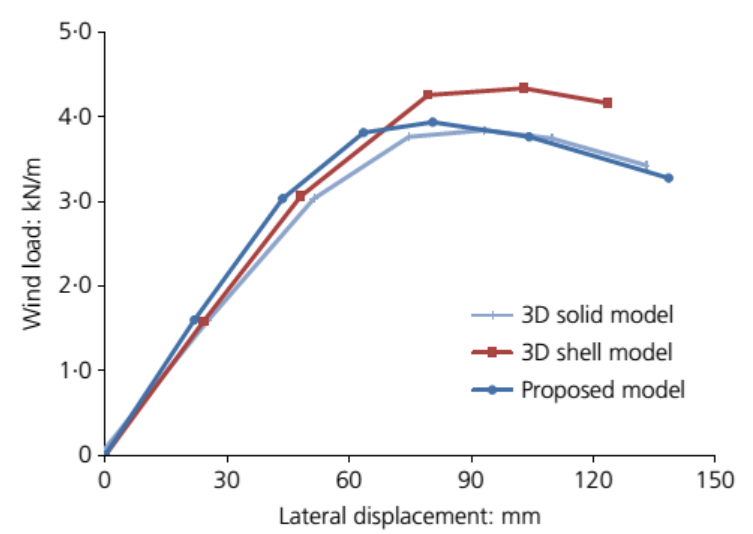

Figure 56 - Influence of different finite element on nonlinear analysis, (LEE et al., 2017b)

The model with beam elements was capable of simulating the behavior of the solid element accurately. Then, wind load, initial imperfections, and span length were evaluated through this model.

With a model considering the same hypothesis, (KRAHL; CARRAZEDO; EL DEBS, 2017) simulated the experimental results of (HURFF, 2010). The camber due to prestress was accounted. Figure 57 presents the finite element model. 


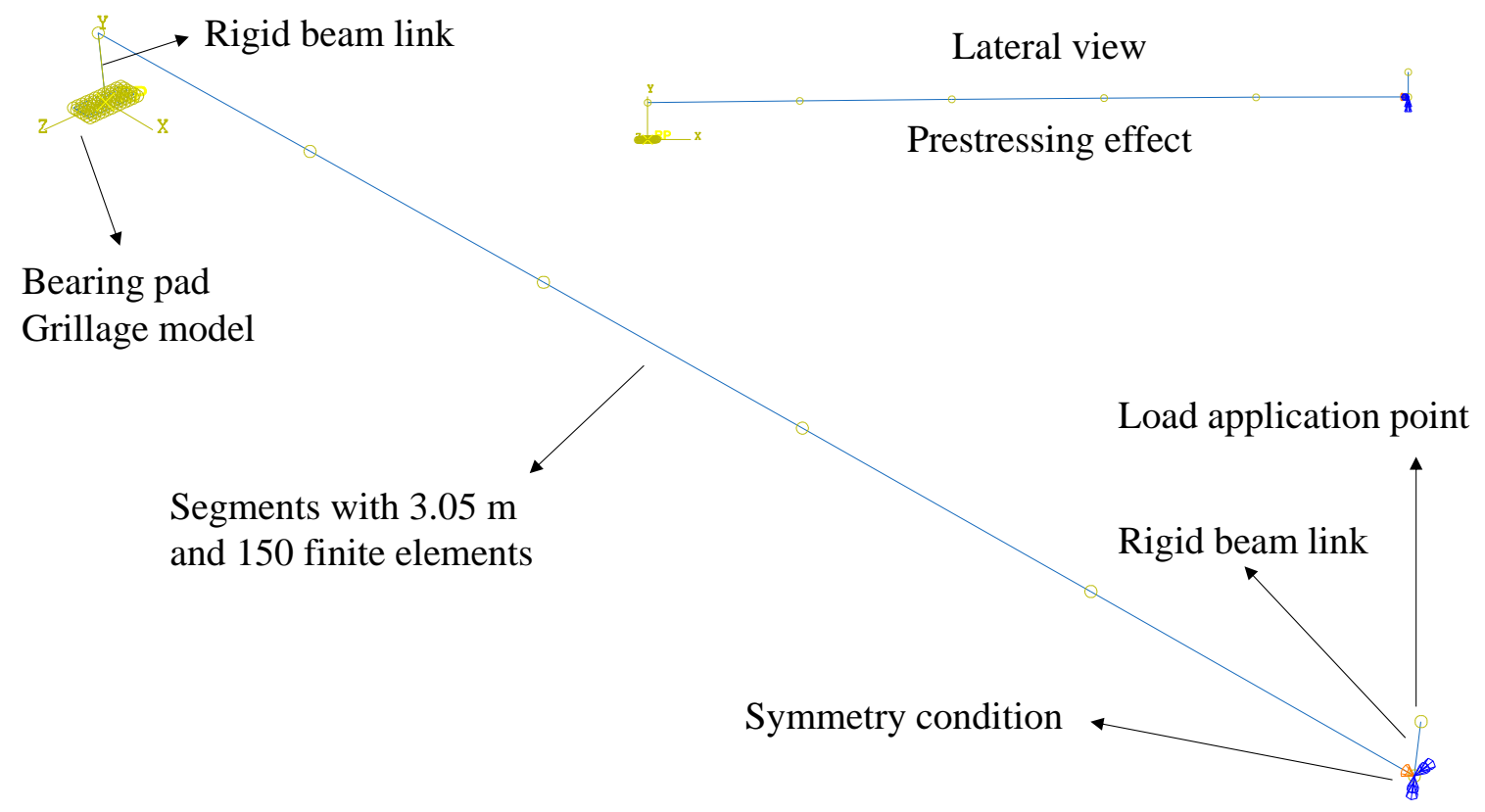

Figure 57 - Model developed by (KRAHL; CARRAZEDO; EL DEBS, 2017)

Figure 58 presents the comparison between experimental and numerical results. The dash and dot curve represents the load-rotation curve for the beam discounting the rigid body rotation, and the dashed line corresponds to the real case.

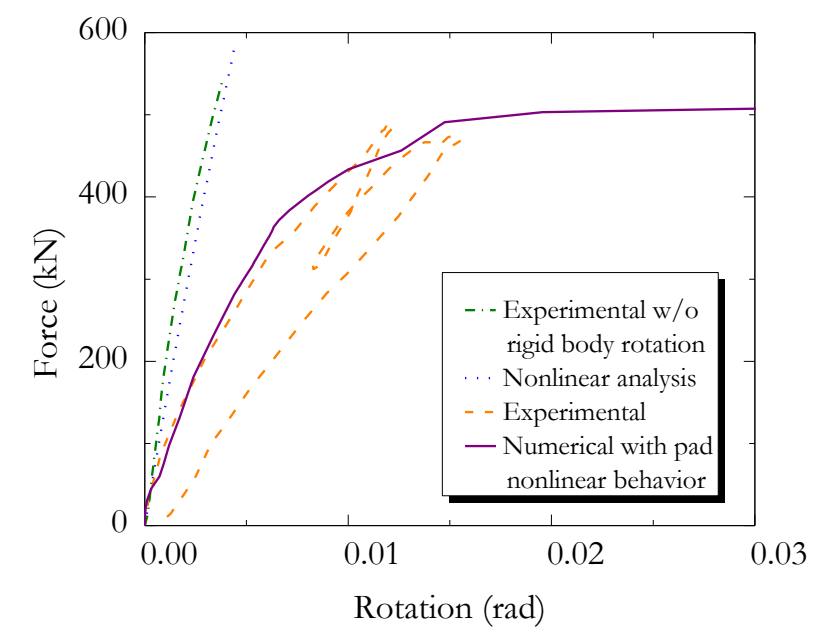

Figure 58 - Comparison of numerical and experimental results

The numerical model could predict both situations with high accuracy for both initial stiffness and peak load. The difference in the instability load was $8.7 \%$. Furthermore, (KRAHL; LIMA; EL DEBS, 2015) developed a parametrical analysis to evaluate the influence of the variation of geometrical parameters, as imperfections, section and span length. No prestressing load and nonlinear material behavior were considered in the numerical models of (LEE et al., 2017b) and (KRAHL; CARRAZEDO; EL DEBS, 2017). 
(CARDOSO; LIMA, 2018) also developed a numerical study for beams supported by bearing pads. One purpose of the paper was to investigate the influence of initial imperfections, concrete and pad behavior on beam stability. The beam section studied was the Type IV of AASHTO with $32 \mathrm{~m}$ of span length. Several values of initial lateral displacements were considered, including recommendations of PCI and Eurocode. The grillage model followed the approach proposed by (HARPER; CONSOLAZIO, 2013) whose stiffness of the tributary area is associated to each spring with different weights, depending on the position. The hypothesis is that at center the pad carries more load than in the perimeter. Also, the springs did not carry tensile load, and the compressive behavior was linear. Furthermore, the concrete beam was modeled with solid finite elements, and a nonlinear geometrical analysis was performed to evaluate possible large displacements. The increase in concrete strength increased the stability capacity of the beams. The authors also verified the high influence of imperfections on the beam behavior. Besides, large initial imperfections can cause the appearance of skewed angles with the pads, which reduces the element capacity. 


\section{EXPERIMENTAL STUDY: MATERIALS AND METHODS}

\subsection{Introduction}

This chapter presents the methodologies to develop the experimental research on the material and the structural element. Also, the materials characterization to develop UHPFRC is presented. Two mixtures were developed, called Batch 1 and 2. The batch 1 was performed without quartz powder. The batch 2 was developed two times. The first was only for material study and to determine the mixture for the beam. The second was developed to produce the UHPFRC beams for the lateral stability research. The beams and the samples for material characterization were not heat treated. Furthermore, the test procedures for material characterization and the study of the lateral stability of beams during lifting are presented in this chapter.

\subsection{Batches}

Two UHPFRC mixtures were developed in the present research called Batch 1 and Batch 2. The fundamental difference was the presence of quartz powder in the second mixture. The first, called Batch 1, was done with cement, silica fume and fine sand as dry components. The mix proportions were based on the study of (SOBUZ et al., 2016), which tested 40 mixture compositions. Even with the differences in the gradations of the constituents, this composition generated a matrix with $131.5 \mathrm{MPa}$ of compressive strength with heat curing. Besides, the investigations with this batch were mainly focused on a material level. Then, uniaxial compressive and direct tensile tests and four-point bending tests were carried out, all under cyclic loading. Table 7 presents the number of specimens for Batch 1. The Batch 2 was utilized to construct the two UHPFRC beams that were tested under lifting. However, before casting the beams, a study was performed to determine the mixture proportions for Batch 2. At the same time of the beam casting, six samples for uniaxial compression tests and four samples for four-point bending tests were cast for material characterization. 
Table 7 - Number of specimens of Batch 1

\begin{tabular}{cccc} 
& \multicolumn{3}{c}{ Fiber content } \\
\hline Test & $0 \%$ & $1 \%$ & $2 \%$ \\
\hline Tension & 4 & 5 & 5 \\
Compression & 6 & 6 & 9 \\
Bending & 2 & 2 & 2 \\
\hline
\end{tabular}

\subsection{UHPFRC mix}

The mix designed by (SOBUZ et al., 2016) was 1:1:0.266:0.233. Then, UHPC for Batch 1 was produced with $850 \mathrm{~kg} / \mathrm{m}^{3}$ of ASTM type III cement (or CPV-ARI in Brazil), $226 \mathrm{~kg} / \mathrm{m}^{3}$ of silica fume (fineness of $20000 \mathrm{~m}^{2} / \mathrm{kg}$ and density of $2220 \mathrm{~kg} / \mathrm{m}^{3}$ ), $850 \mathrm{~kg} / \mathrm{m}^{3}$ of fine sand (grain size less than $0.42 \mathrm{~mm}$ ), $170 \mathrm{~kg} / \mathrm{m}^{3}$ of water and $76.5 \mathrm{~kg} / \mathrm{m}^{3}$ of polycarboxylate-based superplasticizer (density of $1060 \mathrm{~kg} / \mathrm{m}^{3}$ ). The particle size distribution of a combination of the dry materials is compared to the modified Andreasen and Andersen model (A\&Am), equation (43), through the program developed by (HANSSEN, 2012).

$$
P_{t a r}(D)=\frac{D^{q}-D_{\text {min }}^{q}}{D_{\text {máx }}^{q}-D_{\text {min }}^{q}}
$$

Where $P(D)$ is the fraction of the total solids being smaller than the particle size $\mathrm{D}$ and $q$ is the distribution modulus. For UHPFRC, (YU; SPIESZ; BROUWERS, 2014) recommended $q=0.23$. The curve of the model represents an optimal particle size distribution of all the materials in the mix theoretically. For Batch 2, quartz powder was added, and an experimental study was made to determine the mix proportion based on the best mechanical performance achieved. The mixes were called UHPC-1 (1:1.1:0.25:0.3), UHPC-2 (1:1:0.25:0.3), and UHPC-3 (1:1.1:0.25:0.5).

Figure 59 presents the comparison between the theoretical particle size distribution and for Batch 1 and Batch 2 (UHPC-1). The axis of particle size is in log scale. 


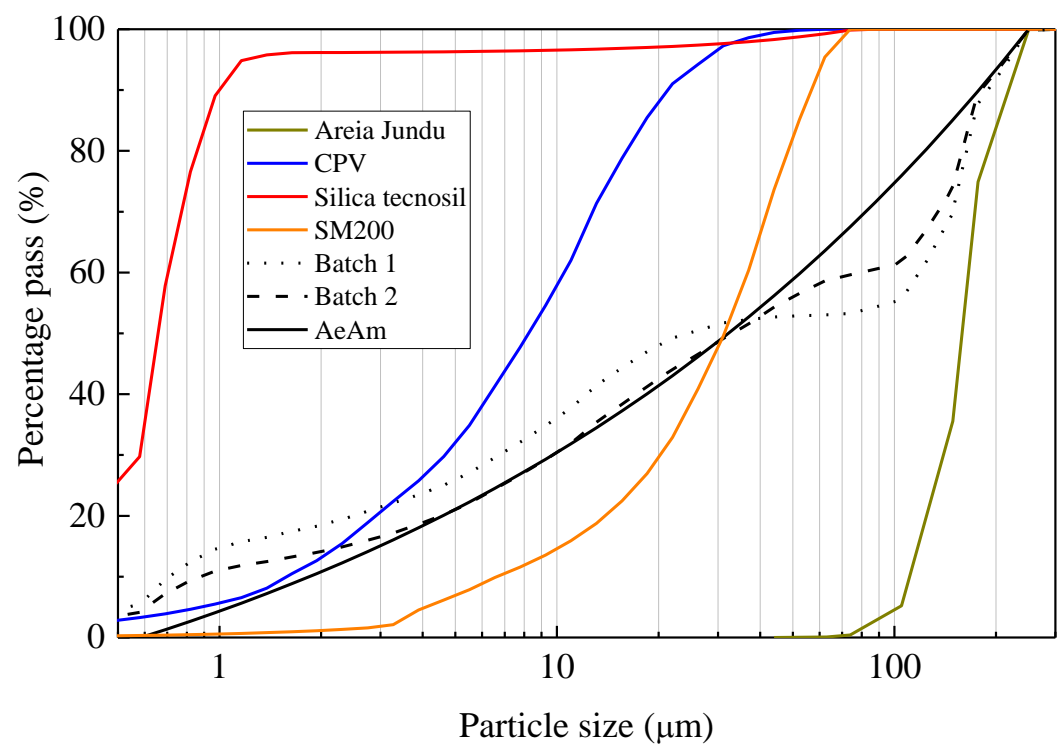

Figure 59 - Comparison of A\&Am and the actual particle size distribution of studied batches

This comparison enables to visualize the level of packing of the mixes. The addition of quartz powder increased the packing in the range between $20 \mu \mathrm{m}$ and $200 \mu \mathrm{m}$.

Then, the mix proportion chosen for UHPFRC was based on the mechanical performance at 28 days, without heat treatment, because the casted beams are not heat treated. Thus, the higher strength and modulus of elasticity at 28 days of the mix UHPC-3 decided the choice for this mix. The results are presented in the topic 7.2.

The slump flow test was performed according to ASTM C1611 / C1611M - 14 to determine the fresh properties of UHPFRC. The determined average flow diameter for UHPC-3 was $675 \mathrm{~mm}$.

Scanning electron microscopy (SEM) was utilized to investigate the shape of the mix constituents. Figure 60 (a) and (b) and Figure 61 (a) and (b) present respectively the images of the cement, silica fume, quartz powder and fine sand. A scale is also presented on each image. The particle morphologies were obtained by scanning electron microscopy (SEM) at the Central of Instrumental Chemical Analysis of the Institute of Chemistry of São Carlos (CAQI / IQSC / USP) in a ZEISS LEO 440 (Cambridge, England) with OXFORD detector (model 7060), electron beam of $20 \mathrm{kV}$, current of 2.82A and probing of 200pA. The samples were covered with $6 \mathrm{~nm}$ of gold in a BAL-TEC MED 020 metalized coating system (BALTEC, Liechtenstein) and kept in a desiccator until the moment of analysis. Metallization conditions: chamber pressure $=2.00 \times 10-2$ mbar; current $=60 \mathrm{~mA}$; deposition rate $0.60 \mathrm{~nm} /$ s). 

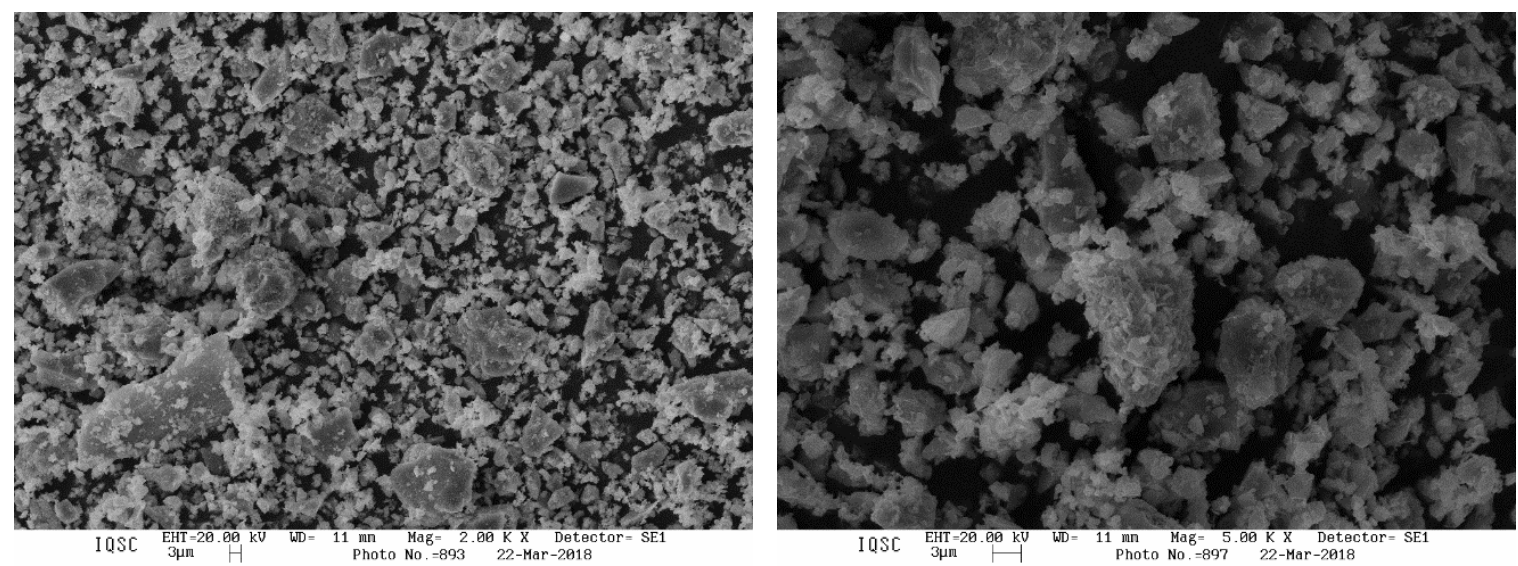

(a)
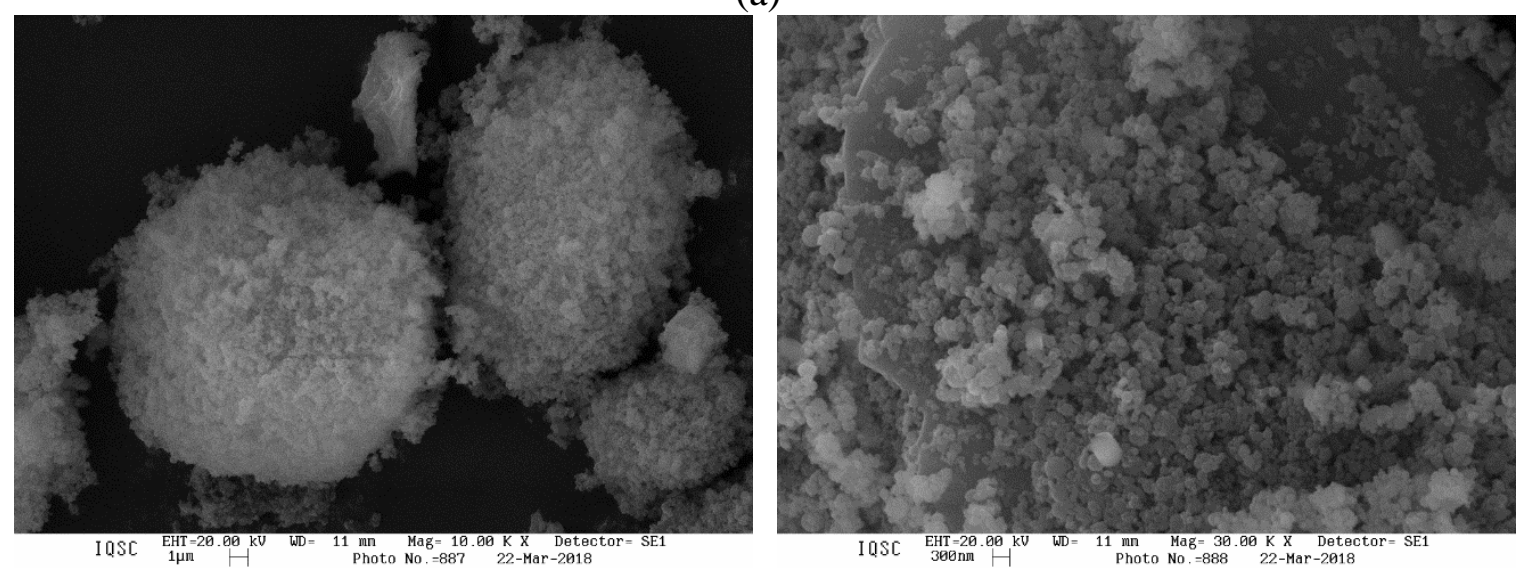

(b)

Figure 60 - (a) cement and (b) silica fume 

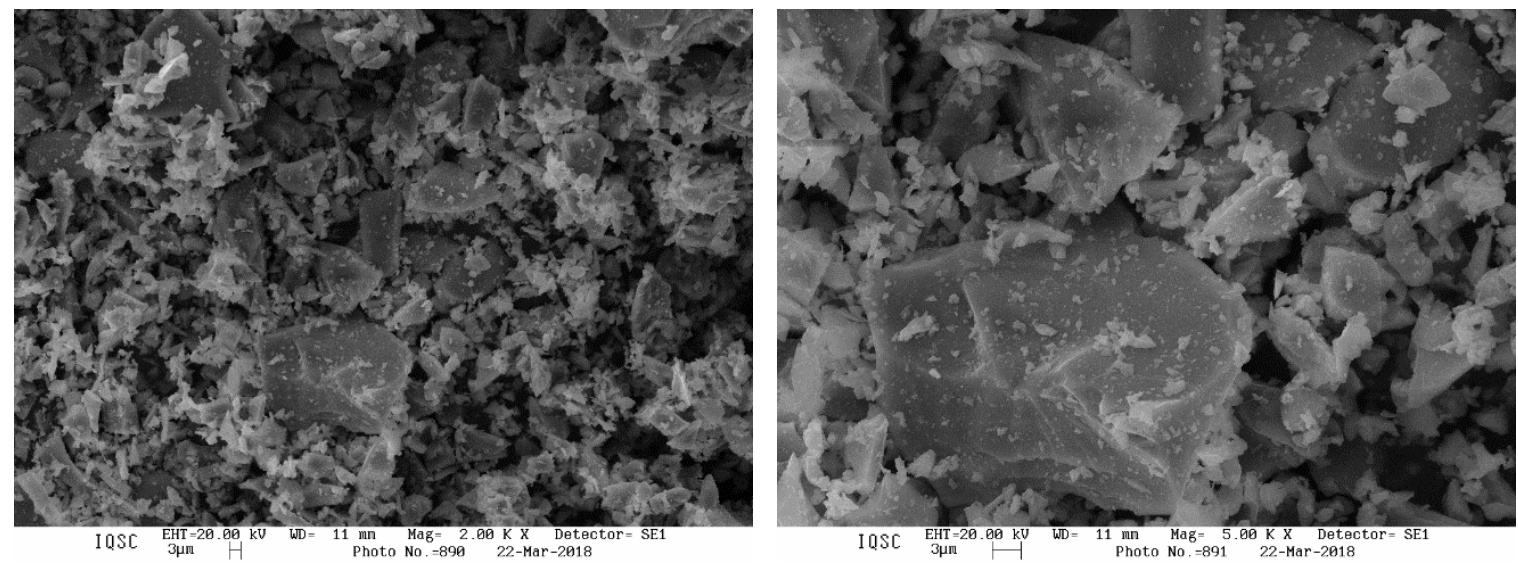

(a)
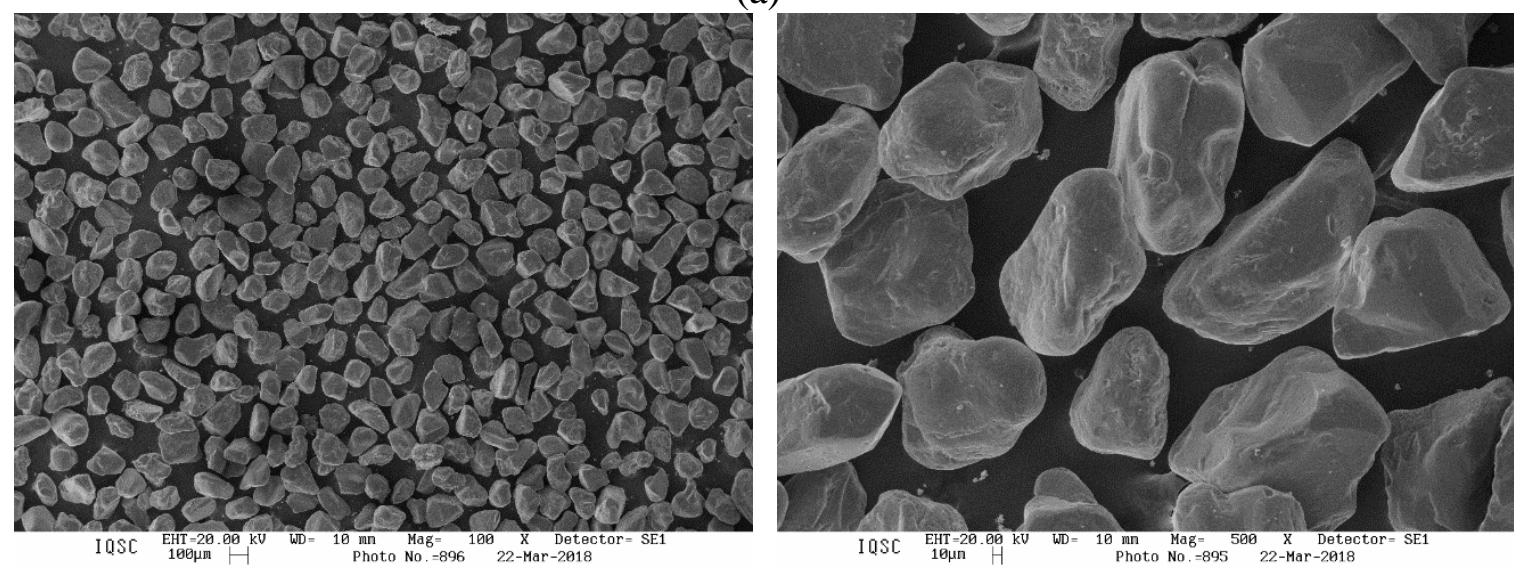

(b)

Figure $61-(a)$ quartz powder and (b) fine sand

Particle shape mechanisms can increase the flowability of the mix. From a qualitative evaluation, it can be said that cement and quartz powder present angular particles and silica fume and the sand are rounded. Thus, future research can consider the addition of more rounded constituents, like fly ash, to increase flowability (AGHDASI et al., 2015), and strength (CHEN; GAO; REN, 2018). Besides, Figure 61 (b) showed the tendency of silica fume to agglomerate due to the attraction forces caused by the high surface area.

The chemical composition of the components utilized to produce UHPC was determined through the X-ray fluorescence technic. The results were expressed regarding oxides, see Table 8 . 
Table 8 - Composition of cement and silica fume

\begin{tabular}{lcc}
\hline Composition \% & Cement type III & Silica fume \\
\hline $\mathrm{CaO}$ & 64.318 & 0.521 \\
$\mathrm{SiO}_{2}$ & 15.400 & 95.722 \\
$\mathrm{Na}_{2} \mathrm{O}$ & 5.188 & 1.552 \\
$\mathrm{Fe}_{2} \mathrm{O}_{3}$ & 3.594 & - \\
$\mathrm{SO}_{3}$ & 3.154 & 0.322 \\
$\mathrm{MgO}$ & 2.718 & 0.438 \\
$\mathrm{Al}_{2} \mathrm{O}_{3}$ & 2.219 & 0.550 \\
$\mathrm{In}_{2} \mathrm{O}_{3}$ & 1.012 & - \\
$\mathrm{Sb}_{2} \mathrm{O}_{3}$ & 0.821 & - \\
$\mathrm{K}_{2} \mathrm{O}$ & 0.713 & 0.761 \\
$\mathrm{TiO}_{2}$ & 0.387 & - \\
$\mathrm{SrO}_{\mathrm{SnO}}$ & 0.191 & - \\
$\mathrm{MnO}$ & 0.173 & - \\
\hline
\end{tabular}

For quartz powder and fine sand, the analysis detected respectively $97.5 \%$ and more than $99 \%$ of $\mathrm{SiO}_{2}$.

For Batch 1, three different fiber contents were considered, $0 \%, 1 \%$ and $2 \%$ by total volume of the mix. For $1 \%$ fiber content, $78 \mathrm{~kg} / \mathrm{m}^{3}$ of copper-coated steel fibers with $13 \mathrm{~mm}$ of length and diameter of $0.2 \mathrm{~mm}$ and $157 \mathrm{~kg} / \mathrm{m}^{3}$ for $2 \%$ fiber content. For the Batch 2 , only $2 \%$ of copper-coated steel fibers were utilized. The tensile strength and elastic modulus of fibers are of $2850 \mathrm{MPa}$ and $200 \mathrm{GPa}$, respectively. Figure 62 (a) presents an SEM image of the fiber surface and Figure 62 (b) and (c) an enlarged image that shows the copper deposited on the metal alloy. Also, Figure 62 (d) shows the results of an analysis of Dispersive Energy Spectroscopy (DES), which enabled to determine the constituents of the fiber. The DES analysis detected $66.2 \%$ of Iron $(\mathrm{Fe}), 22.05 \%$ of Copper $(\mathrm{Cu})$ and $11.80 \%$ of Zinc ( $\mathrm{Zn})$. 


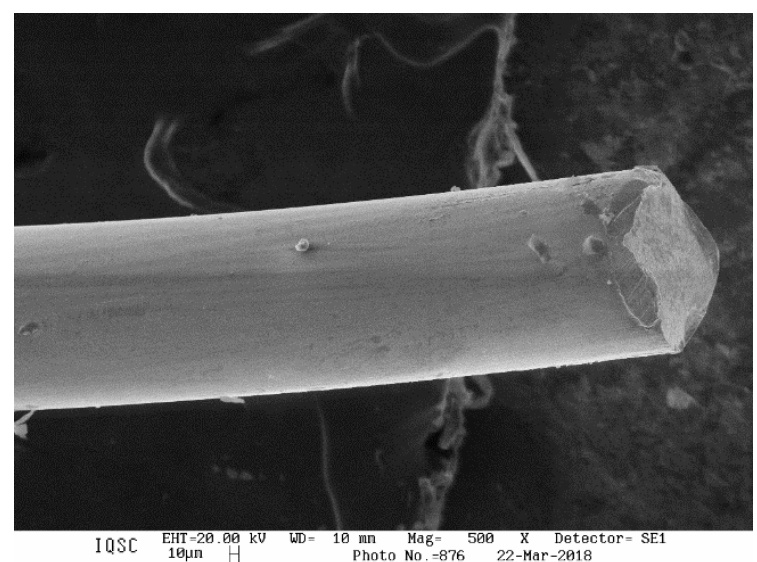

(a)

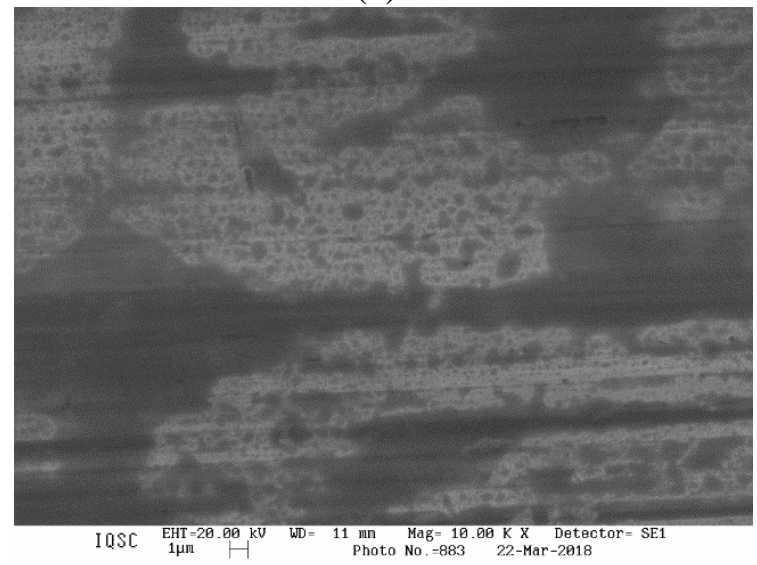

(c)

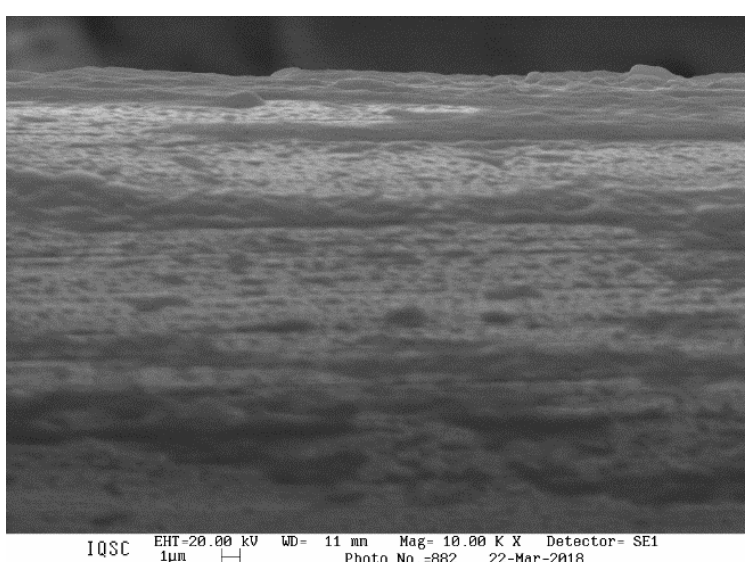

(b)

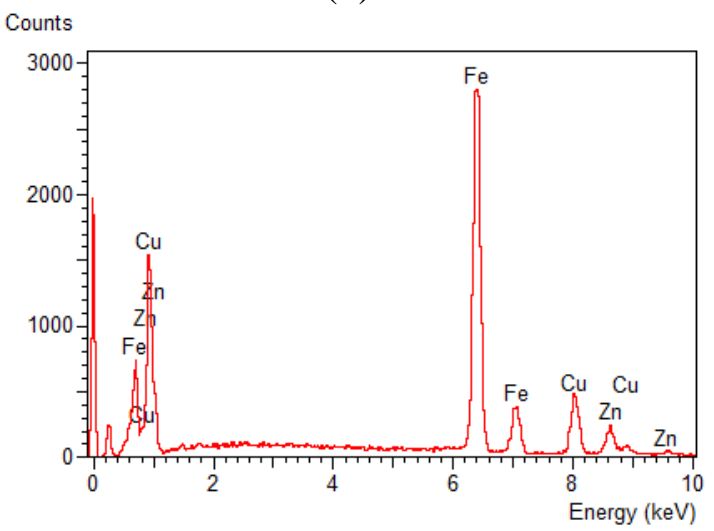

(d)

Figure 62 - SEM images of the (a) end of the fiber, (b), (c) roughness of the surface and the (d) chemical constituents presented in the fiber

\subsection{Mixing, sample preparation, and curing process}

For Batch 1, a portable rotating drum mixer of $0.2 \mathrm{~m}^{3}$ was used. Firstly, all dry components, excepting fibers, were mixed for $5 \mathrm{~min}$ until a homogeneous mixture was obtained. Then, water and superplasticizer were added, and concrete was mixed during additional $20 \mathrm{~min}$ to get the desired workability. Finally, steel fibers were included, and concrete was mixed for more $5 \mathrm{~min}$ to obtain a homogeneous distribution of fibers. Concrete was then molded on a vibrating table to improve densification. Following the casting process, all samples were stored in a moist chamber for 28 days. After this, the samples were submerged in water for heat treatment for seven days at $70^{\circ} \mathrm{C}$.

For Batch 2, a pan-style mixer with orbital mixing action of $0.35 \mathrm{~m}^{3}$ was used. Firstly, all sand was mixed with half of the water volume for 2.5 min until a homogeneous mixture. Then, all dry components, excepting fibers, were added. After each component is set, the mixer was started for a few seconds to facilitate the mixture during additions. After, water 
and superplasticizer were added, and concrete was mixed during additional 20 min to get the desired workability. Finally, steel fibers were added, and concrete was mixed for more 5 min, which was enough to obtain a homogeneous distribution of fibers. The cylinders and prisms were stored in a moist chamber for 28 days, and the beams were covered by a thick foam, which was kept soaked throughout this time.

The preliminary tests to determine Batch 2 were performed in a pan mixer with volume capacity between 5-10 1. The curing regime was the same as Batch 1 . The specimens were tested under compression in 3, 7, 28 and 35 days. Besides the unreinforced UHPC, an additional study was performed to evaluate the mechanical compressive performance of UHPFRC with different fiber contents.

\subsection{Uniaxial compressive test of UHPFRC}

The compression tests were performed on cylindrical specimens of $50 \mathrm{~mm}$ in diameter and $100 \mathrm{~mm}$ in length. The test was carried out under displacement control in a universal servo-hydraulic machine testing machine with a maximum load capacity of 1500 $\mathrm{kN}$. Besides, an axial hinge was installed on the top of the specimen. Two axial LVDTs (Linear Variable Differential Transformer) were fixed between the steel plates of the test machine. The stiffness of the hinge is much higher than the UHPFRC sample. Thus, the strain occurred at the hinge can be disregarded. The gauge length considered for the LVDTs is $100 \mathrm{~mm}$. After crack localization, these devices enable to measure the post-peak branch. Also, two clip gages were attached to the UHPFRC samples, as presented in Figure 63. Each of these instruments measures longitudinal displacement in the material for a gauge length of $50 \mathrm{~mm}$. The modulus of elasticity is determined from clip gages at a stress level of $40 \%$ of the ultimate load, as recommended by ASTM C469 / C469M-14. 


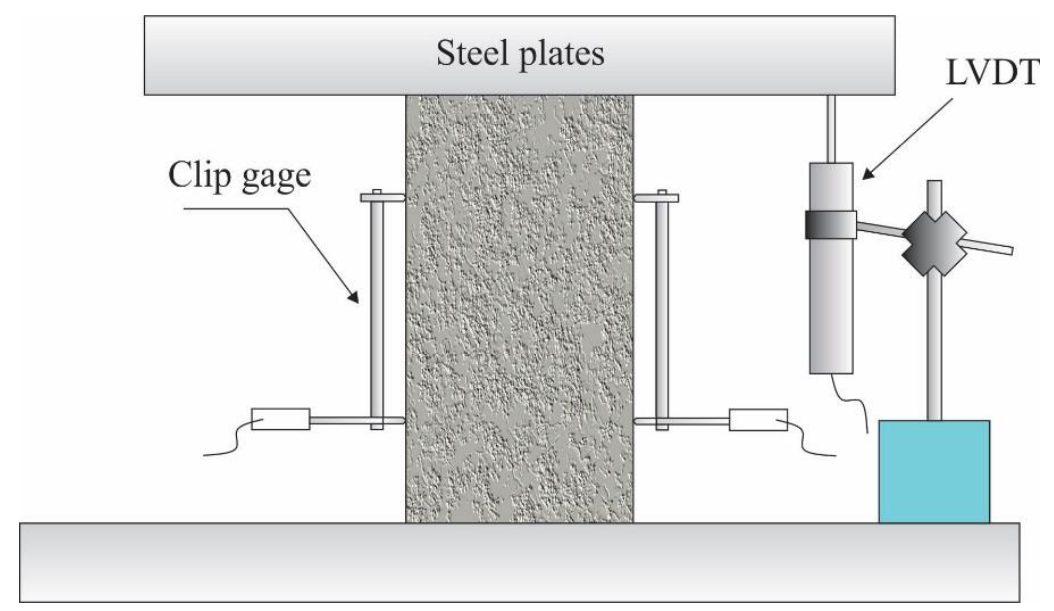

Figure 63 - Compression test set-up

Figure 64 presents a more detailed image of the clip gages. Two points of each clip are fixed at the sample with the distance of $50 \mathrm{~mm}$. One of them is the end of a flexible steel beam where strain gauges are glued to determine the strain correspondent to the gauge length.

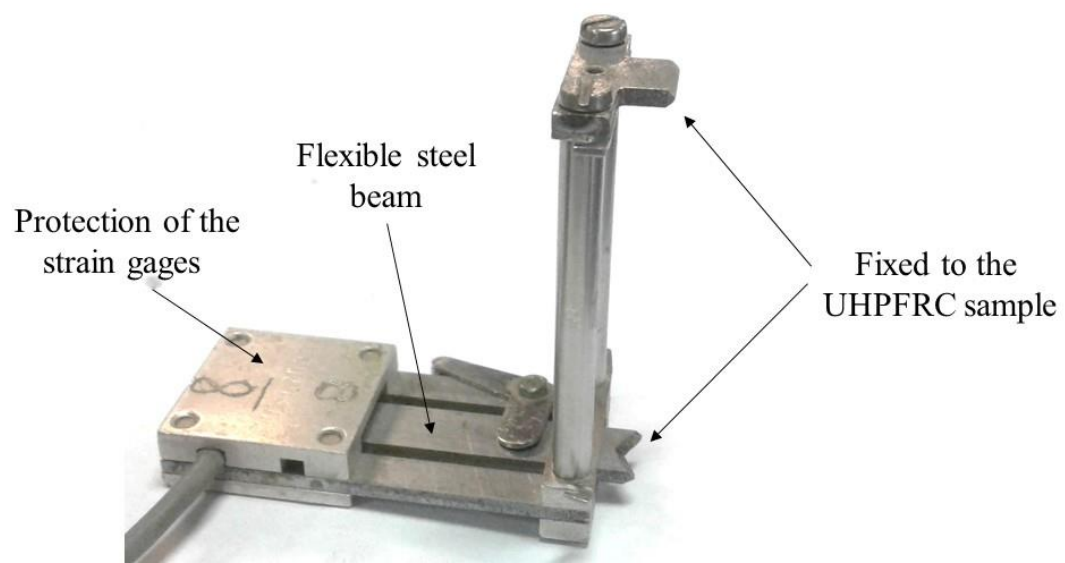

Figure 64 - Details of the clip gage

The displacement applied had the speed of $0.005 \mathrm{~mm} / \mathrm{s}$. For gauge length of the LVDT, the strain rate $5 \times 10^{-5} \mathrm{~s}^{-1}$. The initial inclination of the stress-strain curves obtained from clip and LVDT are different because the LVDT measurements account for accommodations between the machine plates and specimen. Equation (44) enables to correct such effect, (OSORIO; BAIRÁN; MARÍ, 2013),

$$
\varepsilon_{1}=\varepsilon_{1, L V D T}-\frac{\left(E_{\text {clip }}-E_{L V D T}\right)}{E_{c l i p} E_{L V D T}} \sigma_{1}
$$

Where $\varepsilon_{l, L V D T}$ is the average axial strain measured by the LVDTs, $\sigma_{l}$ is the axial stress, $E_{c l i p}$ and $E_{L V D T}$ are the modulus of elasticity obtained from the clip and LVDTs 
readings. Figure 65 presents the conversion performed by equation (44) to the effective stiffness of the sample.

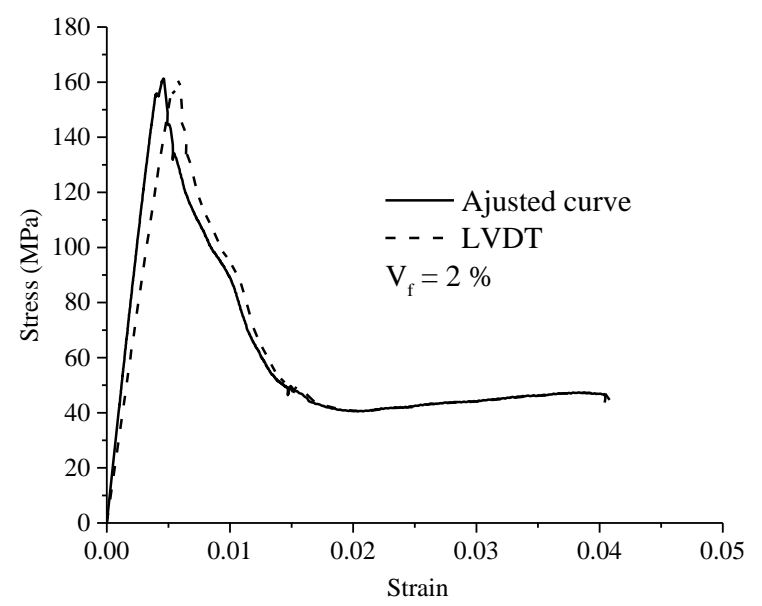

Figure 65 - Conversion of the LVDT measurements by equation (1)

\subsection{Uniaxial tensile test of UHPFRC}

Dog-bone-shaped specimens were tested in an electronic universal testing machine under displacement control. The geometry of samples and testing apparatus are presented in Figure 66. The loading-unloading steps were applied at a constant speed of $0.016 \mathrm{~mm} / \mathrm{s}$. Axial displacement was measured over $80 \mathrm{~mm}$ in length at the central portion of the sample. To this end, two linear variable differential transformers (LVDTs) were positioned on opposite faces of the specimen. With this gauge length, the strain rate resulted in $0.0002 \mathrm{~s}^{-1}$.

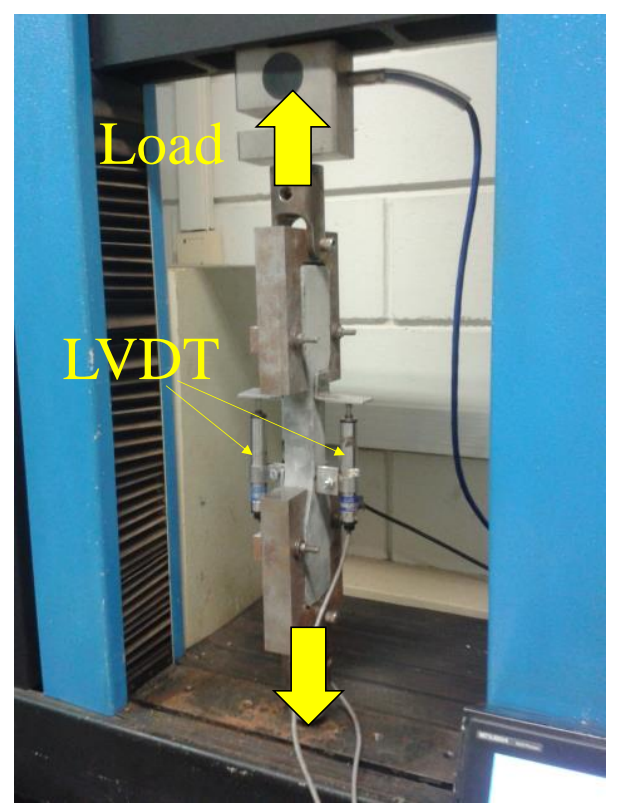

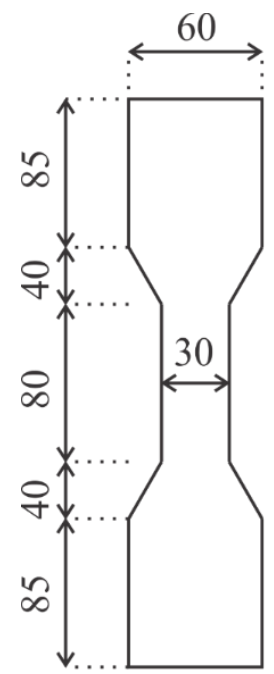

Front view

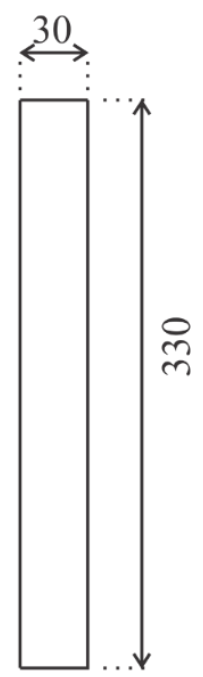

Side view

(dimensions: $\mathrm{mm}$ )

Figure 66 - Test setup for the direct tensile test 


\subsection{Four point bending test of UHPFRC}

Third-point bending tests were carried out according to ASTM C1609 / C1609M-12 in a servo-controlled testing machine. Net deflection of the center of the prism was measured and used to maintain a fixed speed of loading/unloading of $0.01 \mathrm{~mm} / \mathrm{s}$. For this, a yoke shown in Figure 67 was clamped at mid-depth of the prism over the supports. Two LVDT were attached to this jig so that net deflection at the center of the prism could be measured. This procedure allows measurement of load-displacement curve beyond the cracking load for UHPFRC specimens. The samples were rotated $90^{\circ}$ about the longitudinal axis to the casting position, reducing surface roughness for application of load and the possible effect of segregation.
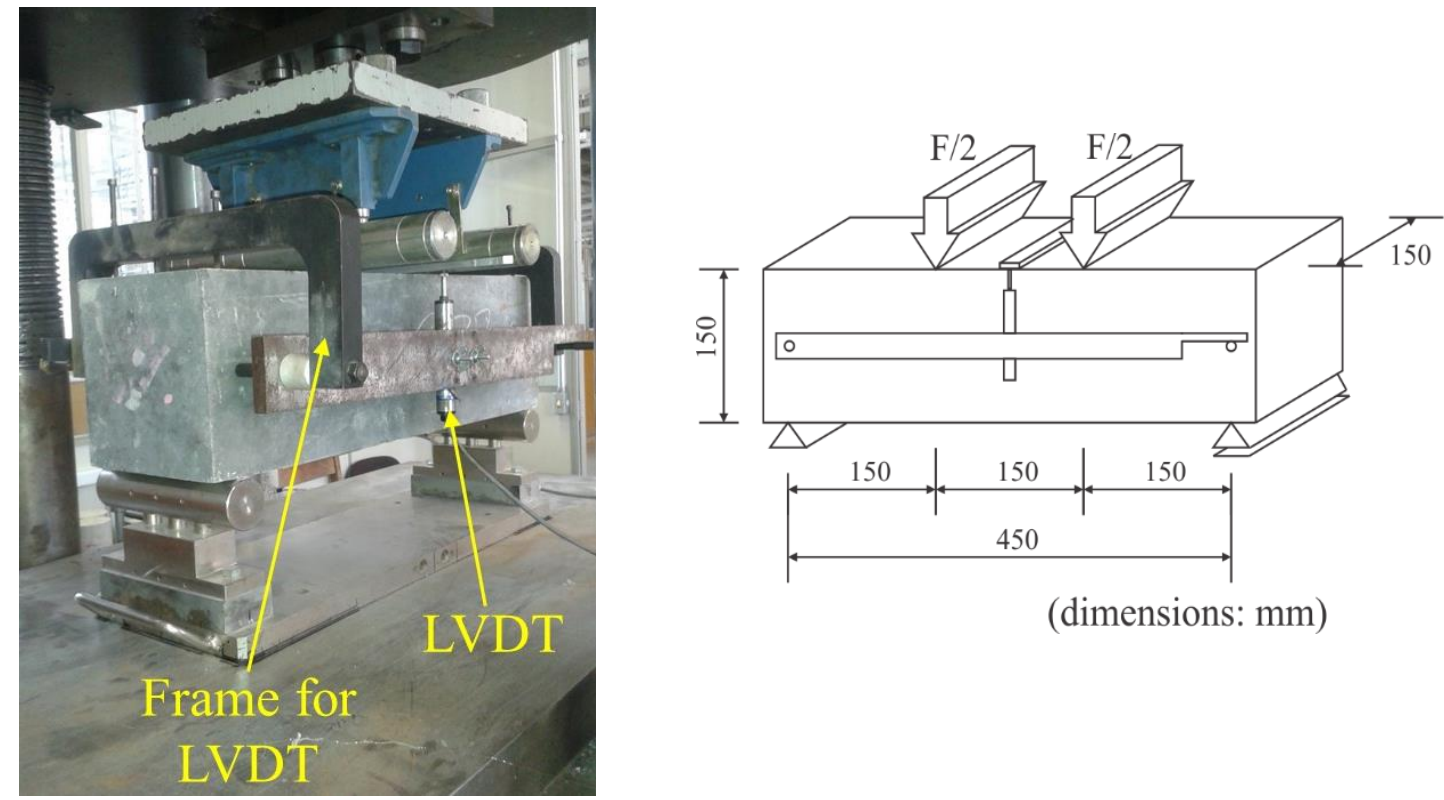

Figure 67 - Test setup for the four-point bending test

\subsection{Loading cycles and experimental damage measurement}

In cyclic tensile tests, the loading/unloading process was performed under displacement control. For the multiple cracking stage, the unloading steps were done at each $0.05 \mathrm{~mm}$, see Figure 68 (a) and (b). Then, in the softening branch, after the appearance of a macrocrack, the interval between cycles was $0.5 \mathrm{~mm}$. In each unloading cycle, the load was decreased to a value close to zero. Therefore, the load was not removed entirely to avoid loss of contact between the grip and the sample. Besides, the unloading speed was the same as the loading speed. 


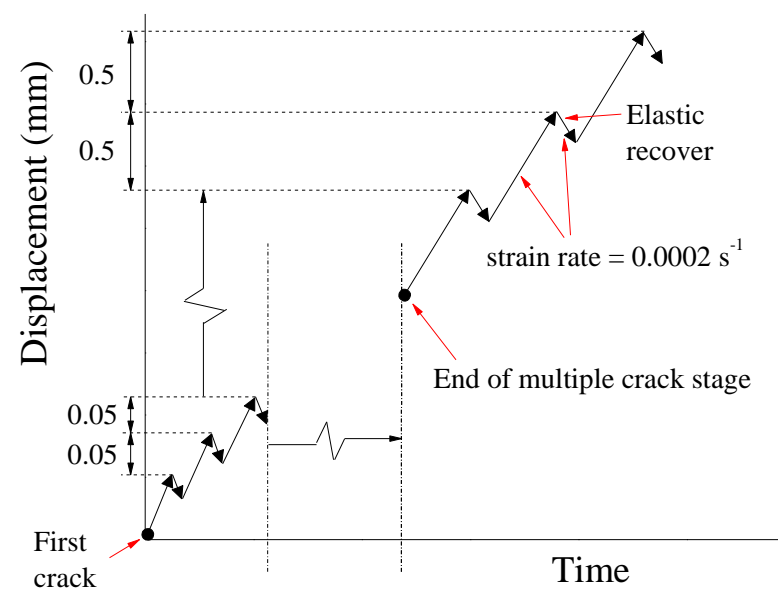

(a)

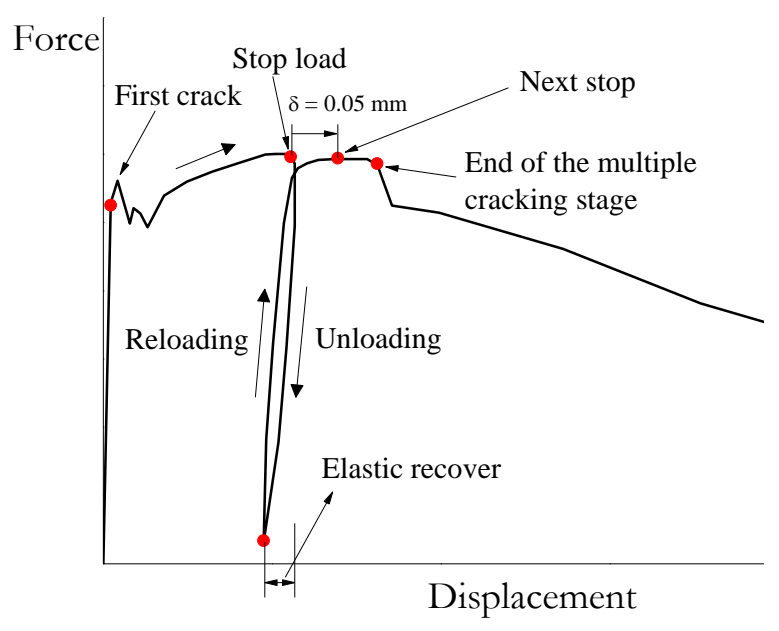

(b)

Figure 68 - Cyclic loading procedure for tension tests

For compression, unloading/reloading cycles were performed manually under displacement control after the peak load at each $0.1 \mathrm{~mm}$. Similarly, specimens were not unloaded entirely to avoid loss of contact with the testing machine and possible accommodations that would interfere with the results. Load cycling procedure is illustrated in Figure 69 (a) and (b). For the third-point bending cyclic test a similar procedure was carried out but with $0.5 \mathrm{~mm}$ of displacement between cycles.

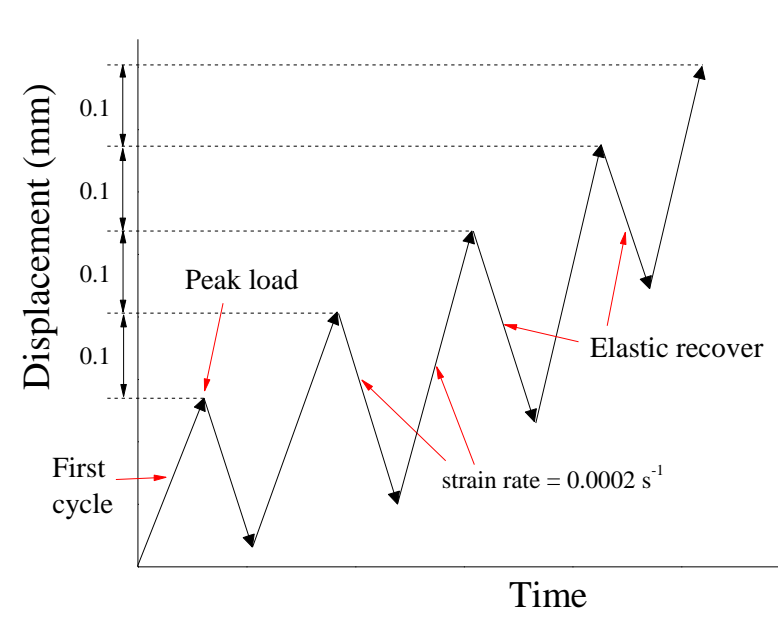

(a)

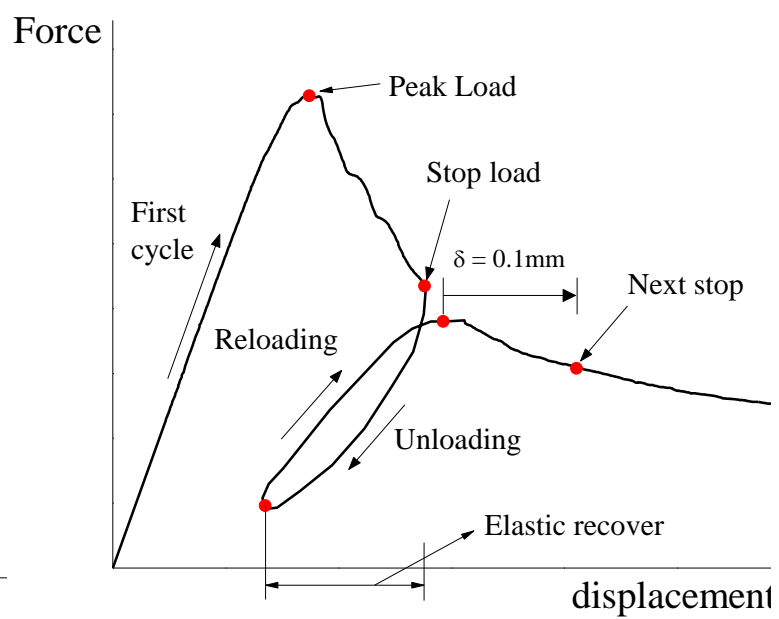

(b)

Figure 69 - Cyclic compression test procedure

\subsection{Damage quantification through cyclic tests}

Cement-based materials present permanent strains and stiffness degradation in the inelastic regime. In this sense, a constitutive model must couple plasticity and damage mechanics to simulate such characteristic behavior. Figure 70, presents the differences between plasticity, damage, and plastic-damage models. The last is considered in the present 
research due to the accordance with experimental results. Experimentally, damage and plastic strains can be recorded through cyclic tests.

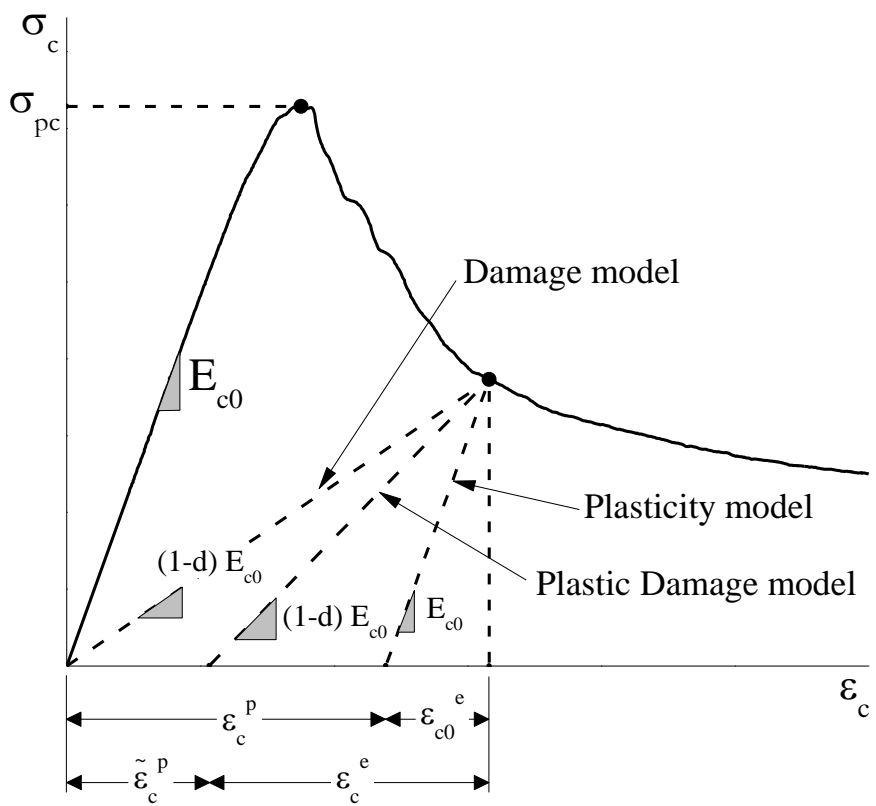

Figure 70 - Difference between plastic, damage and plastic-damage models

From the Figure 70, during the cyclic tests, the degradation is determined as the inclination of the unloading/reloading curve. Then, the constitutive equation for plasticdamage models is:

$$
\sigma=(1-d) E_{c 0}\left(\varepsilon-\tilde{\varepsilon}^{p}\right)
$$

Where $d$ is the internal damage variable, $E_{c 0}$ is the initial tangent modulus of elasticity and $\tilde{\varepsilon}^{p}$ is the equivalent plastic strain. The damage variable enables to represent the stiffness degradation of the composite in both tension $d_{t}$ and compression $d_{c}$. From damage mechanics, (LEMAITRE; CHABOCHE, 1990), damage describes the appearance of surface discontinuities in the form of microcracks which can be quantified macroscopically by measuring the variation of the elastic properties of the material. By definition, the damage variable $d$ represents the change in volume/area of the material, i.e., is the relation between the degraded area $S_{d}$ due to inelastic processes relative to the entire area $S$, equation (46).

$$
d=\frac{S_{d}}{S}
$$

Thus, as the solid is stressed beyond the elastic regime, only the remaining area $\bar{S}$ carries the applied load. Therefore, the effective stress $\bar{\sigma}$ is: 


$$
\begin{aligned}
& \bar{S}=S-S_{d} \\
& \bar{\sigma}=\sigma \frac{S}{\bar{S}}=\frac{\sigma}{1-d}
\end{aligned}
$$

Then, accounting for the concept of strain equivalence, (LEMAITRE; CHABOCHE, 1990), the damage variable can be defined as a material property:

$$
\begin{aligned}
& \left(\varepsilon-\tilde{\varepsilon}^{p}\right)=\frac{\bar{\sigma}}{E_{c 0}}=\frac{\sigma}{E_{c}} \\
& E_{c}=(1-d) E_{c 0} \\
& d=1-\frac{E_{c}}{E_{c 0}}
\end{aligned}
$$

Where $E_{c}$ is the actual elastic modulus of the damaged material, and $E_{c 0}$ is the initial elastic modulus, which was also obtained from the experimental curve, according to ASTM C469 / C469M-14. Thus, for a theoretically undamaged state, the damage variable is zero, and for an entirely damaged state, the damage variable is one. With this approach, it is possible to obtain internal damage variables for UHPFRC under tension $d_{t}$ and compression $d_{c}$ from cyclic tests by applying equation (48).

In the case of flexural tests, the stiffness reduction of the prisms was evaluated quantitatively through a global damage index $D_{g}$, which was calculated as

$$
D_{g}=1-\frac{K_{i}}{K_{0}}
$$

Where $K_{0}$ is the elastic stiffness of the structural element and $K_{i}$ is the actual stiffness. These variables are presented in Figure 71.

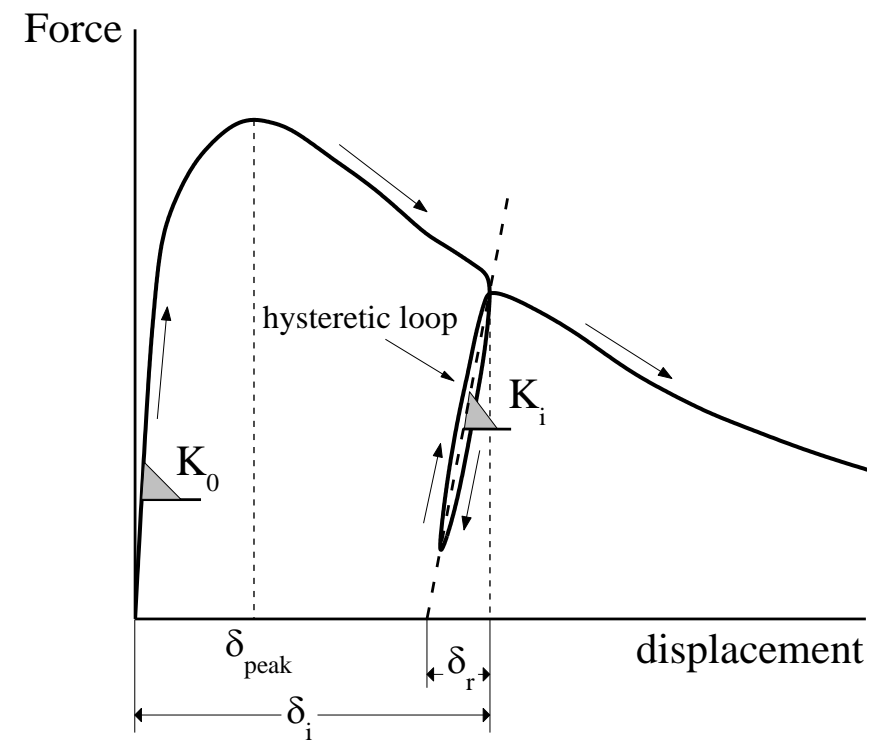

Figure 71 - Schematic representation of global stiffness degradation 


\subsection{Lifting test of UHPFRC beams}

\section{Description of test specimens}

The UHPFRC beams were designed for flexure according to the model proposed by (FEHLING et al., 2014) and were tested at 28 days. The adopted dimensions of the beams were $50 \mathrm{~mm}$ in width, $501 \mathrm{~mm}$ of height and $4050 \mathrm{~mm}$ of total span. Typical loads for residential buildings were considered in the flexural design. The maximum flexural moment for permanent loads was $11.75 \mathrm{kN} . \mathrm{m}$ and for variable loads was $15 \mathrm{kN}$.m. Figure 72 presents the designed reinforcements.

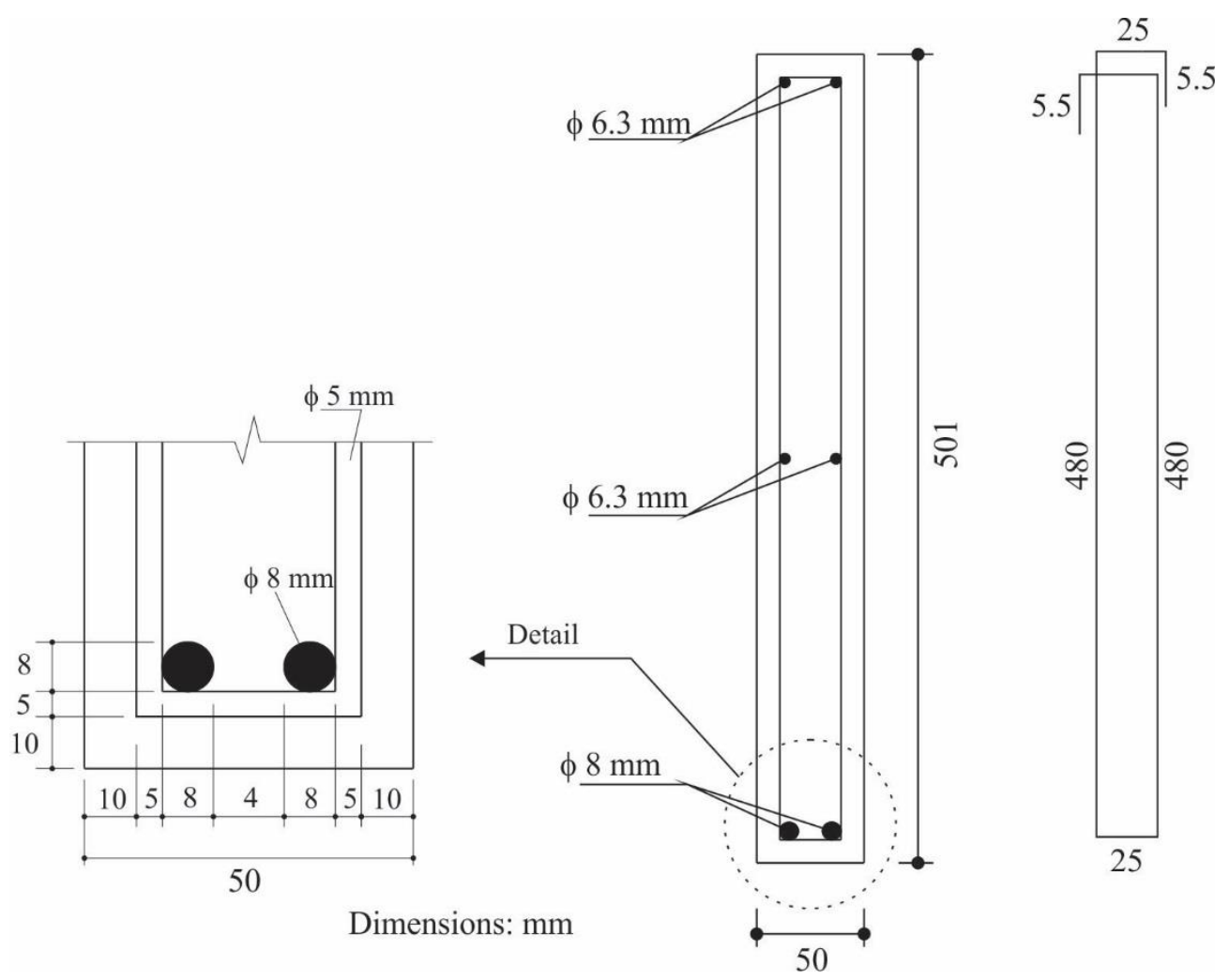

Figure 72 - Cross-section of the designed UHPFRC beam

The beams were cast with the formwork in a toppled position for better densification. No vibration and heat curing were utilized for this batch After demolding, several measurements of the width of the beams were made over each span. The average widths for beam 1 and 2 were respectively $58.5 \mathrm{~mm}$ and $56.8 \mathrm{~mm}$ with standard deviations of 0.21 and 0.30 , respectively.

The lifting loops were positioned $500 \mathrm{~mm}$ from the ends of the beam and with 100 $\mathrm{mm}$ of height. Also, $400 \mathrm{~mm}$ were embedded in concrete in the shape of a loop, see Figure 73. Each cable has a tensile strength of $14.7 \mathrm{kN}$, as specified by the manufacturer. The 
designed longitudinal rebars and stirrups have $500 \mathrm{MPa}$ of specified yielding strength and $210 \mathrm{GPa}$ of the modulus of elasticity with the yielding strain of $2.38 \times 10^{-3}$.

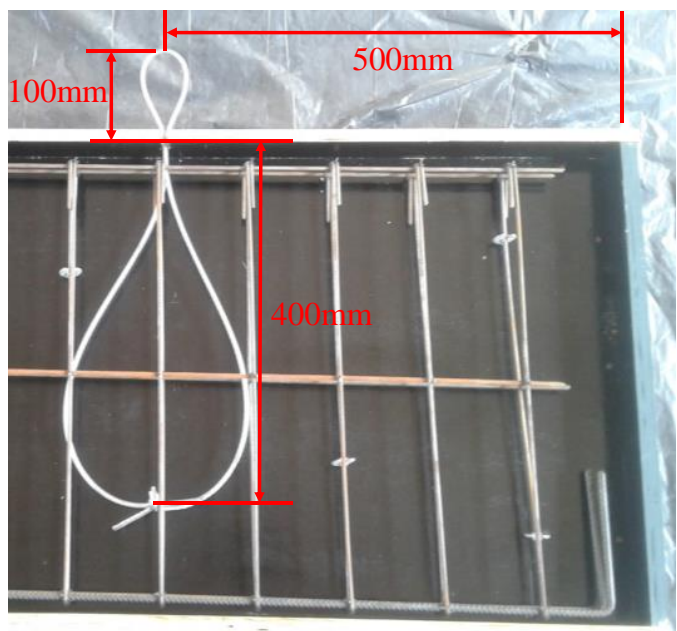

Figure 73 - Lifting loops detail

It is difficult to guarantee that the cables are correctly centered at the beam width due to several reasons, one of them is that the beam present variable width. Furthermore, initial imperfections influence on beam stability and the eccentricity of the cables cause initial rotation. Then, these construction inaccuracies were measured. Figure 74 presents the reference for the eccentricities measurement which is taken from the cables position to the side of the beam opposite to the total station equipment.
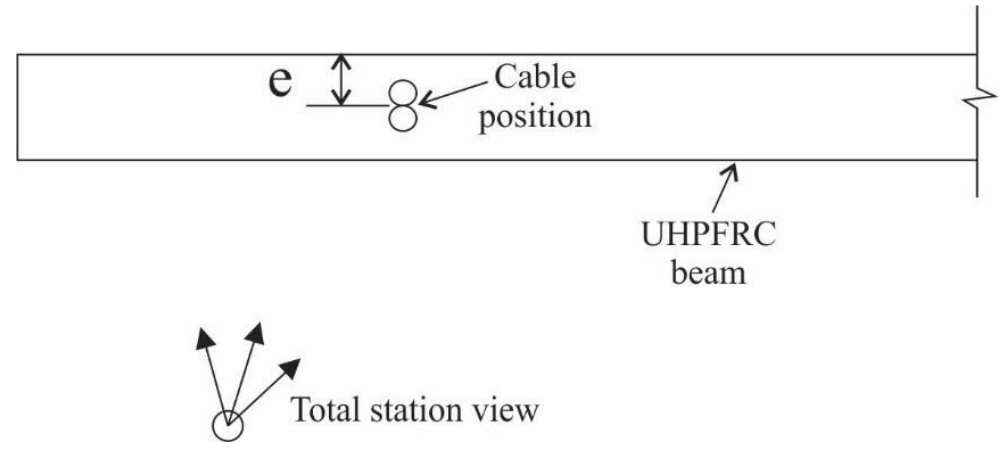

Figure 74 - Reference for the eccentricities measurements

Table 9 presents the measured eccentricities of the lifting cables and the width of the beam at lifting points.

Table 9 - Eccentricities and widths at lifting points

\begin{tabular}{ccc}
\hline & \multicolumn{2}{c}{ Eccentricity (width) - mm } \\
& Beam 1 & Beam 2 \\
\hline Left end & $32(54)$ & $25(57)$ \\
Right end & $24(57)$ & $24(51)$
\end{tabular}




\section{Test setup and procedure}

A hydraulic jack fixed to a steel frame was attached to beam top face to apply a vertical load at midspan. Furthermore, an apparatus was designed to couple a hydraulic jack to the beam and enable the UHPFRC element to rotate over its longitudinal axis freely. Figure 75 presents the test setup.

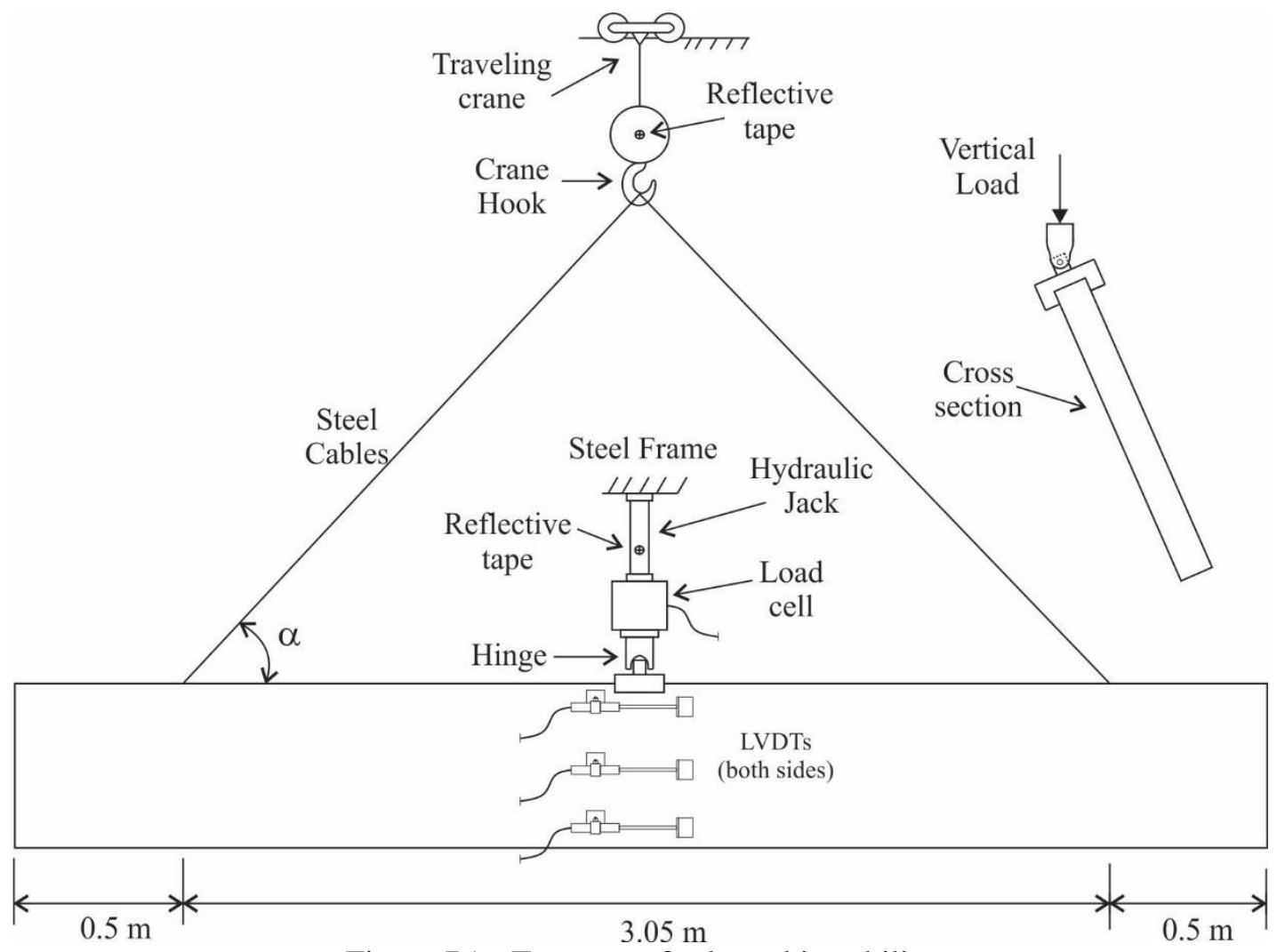

Figure 75 - Test setup for lateral instability

The hinge showed in Figure 75 has two parts, a steel apparatus called the base of the hinge that was screwed in the top face of the beam through two bolts embedded in concrete and the head of the hinge, which in turn is fixed to the load cell. Figure 76 presents the dimensions of the screwed steel device. 

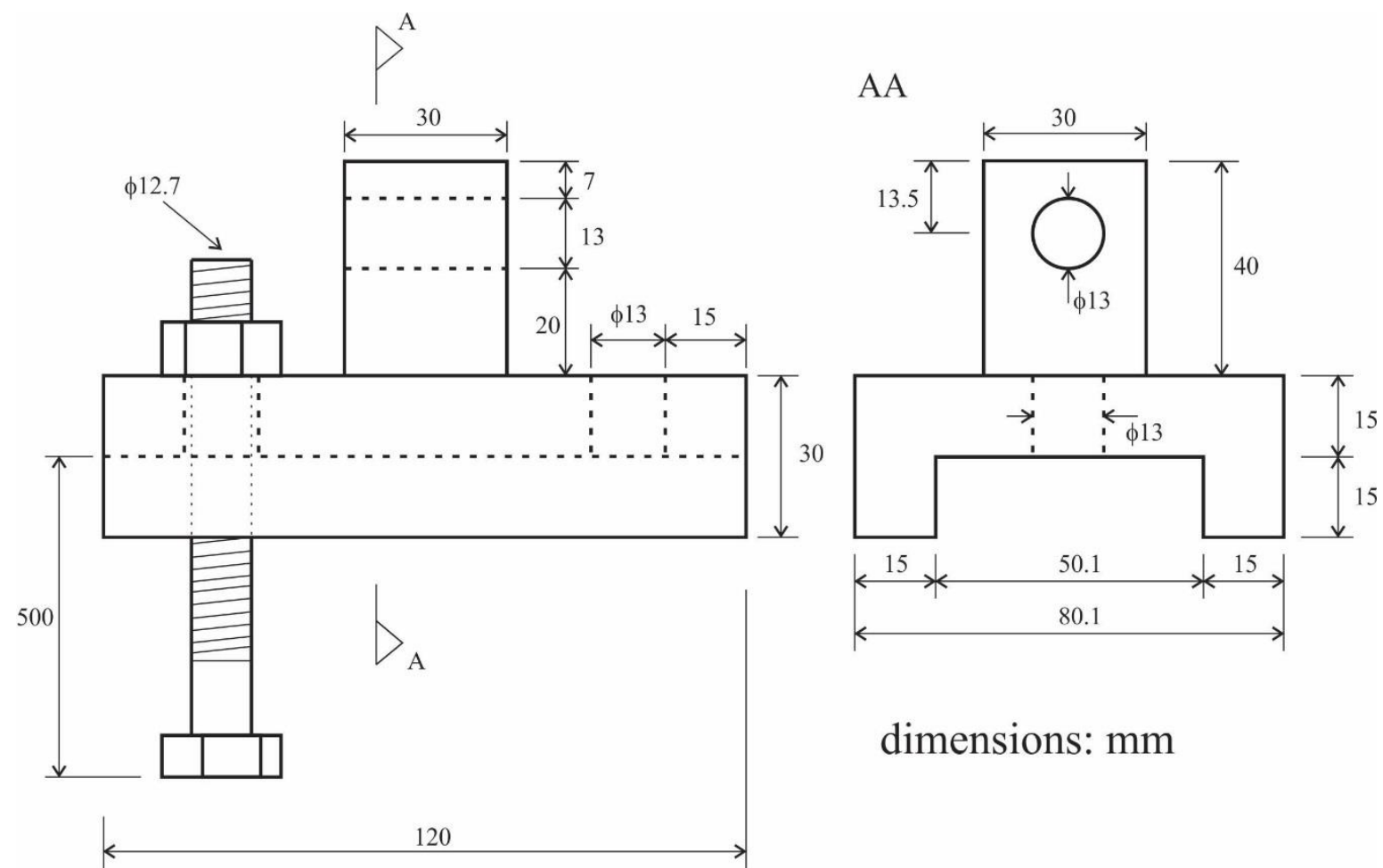

dimensions: $\mathrm{mm}$

Figure 76 - Detail of the base of the hinge

The head of the hinge that is fixed to the load cell, which has $50 \mathrm{kN}$ of nominal capacity. The mechanical actuator has a curse of $304.8 \mathrm{~mm}$, which enables apply large displacements. Figure 77 presents the designed hinge. A steel pin performed the coupling.

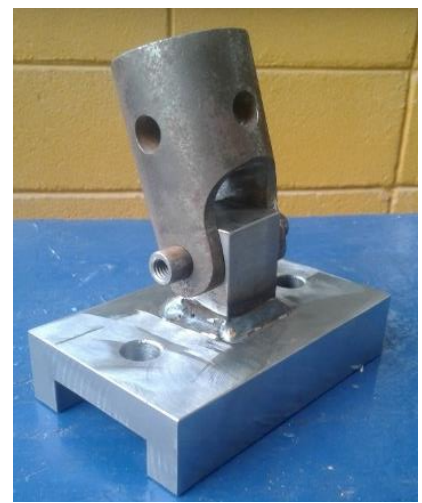

Figure 77 - The designed hinge

As mentioned, two high-strength steel bolts fix the hinge base to the top face of the beam. The bolts had $12.7 \mathrm{~mm}$ in diameter and $500 \mathrm{~mm}$ embedded in concrete and sufficient length to pass by the base of the hinge and be screwed. Figure 78 (a) and (b) show the bolts positioned before casting the UHPFRC beam and the screwed base of the hinge, respectively. 


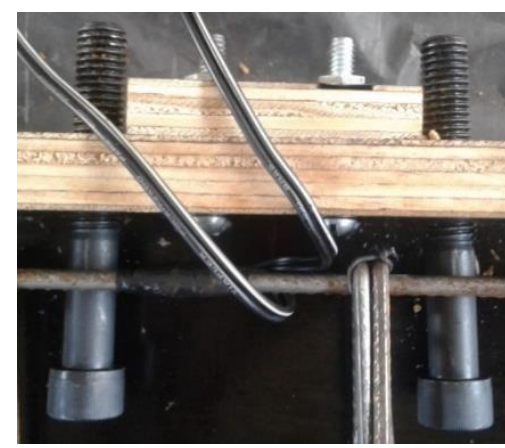

(a)

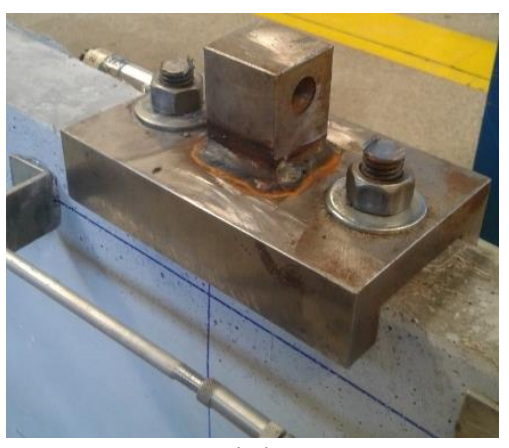

(b)

Figure 78 - (a) Position of the bolts before casting and (b) the base of the hinge screwed at the top face the beam

As shown in the Figure 78 (a), the bolt head was positioned below the longitudinal rebars at the top face of the beam. Also, six LVDTs were positioned at midspan cross-section of the beam to measured longitudinal displacements to be converted into longitudinal strain, and consequently, determine the moment of cracking and the section behavior during loading. Figure 79 presents the positions of the LVDTs on beam 1.

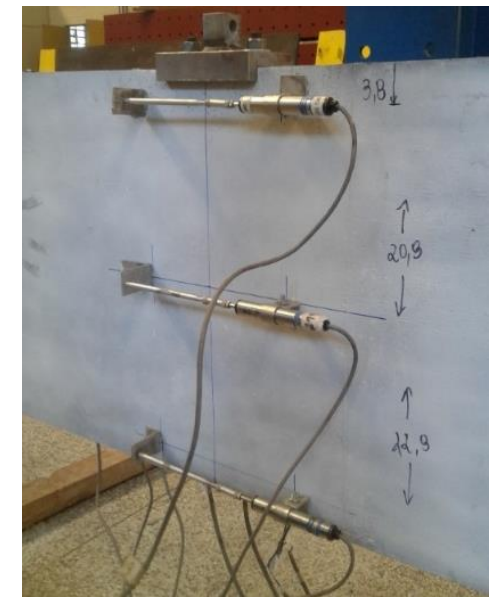

Figure 79 - LVDTs positioned in the midspan cross-section

The gauge length for each LVDT and its respective beam is presented in Table 10.

Table 10 - Gauge lengths for the LVDTs

\begin{tabular}{ccc}
\hline \multirow{2}{*}{ LVDT } & \multicolumn{2}{c}{ Gauge length $(\mathrm{mm})$} \\
\cline { 2 - 3 } & Beam 1 & Beam 2 \\
\hline 1 & 200 & 205 \\
2 & 203 & 205 \\
3 & 204 & 201 \\
4 & 210 & 207 \\
5 & 213 & 197 \\
6 & 222 & 200 \\
\hline
\end{tabular}


Furthermore, the inclination angle of the cables was measured with a protractor after the test setup, as shown in Figure 80. The cables of beam 1 and 2 presented 48 degrees of inclination.
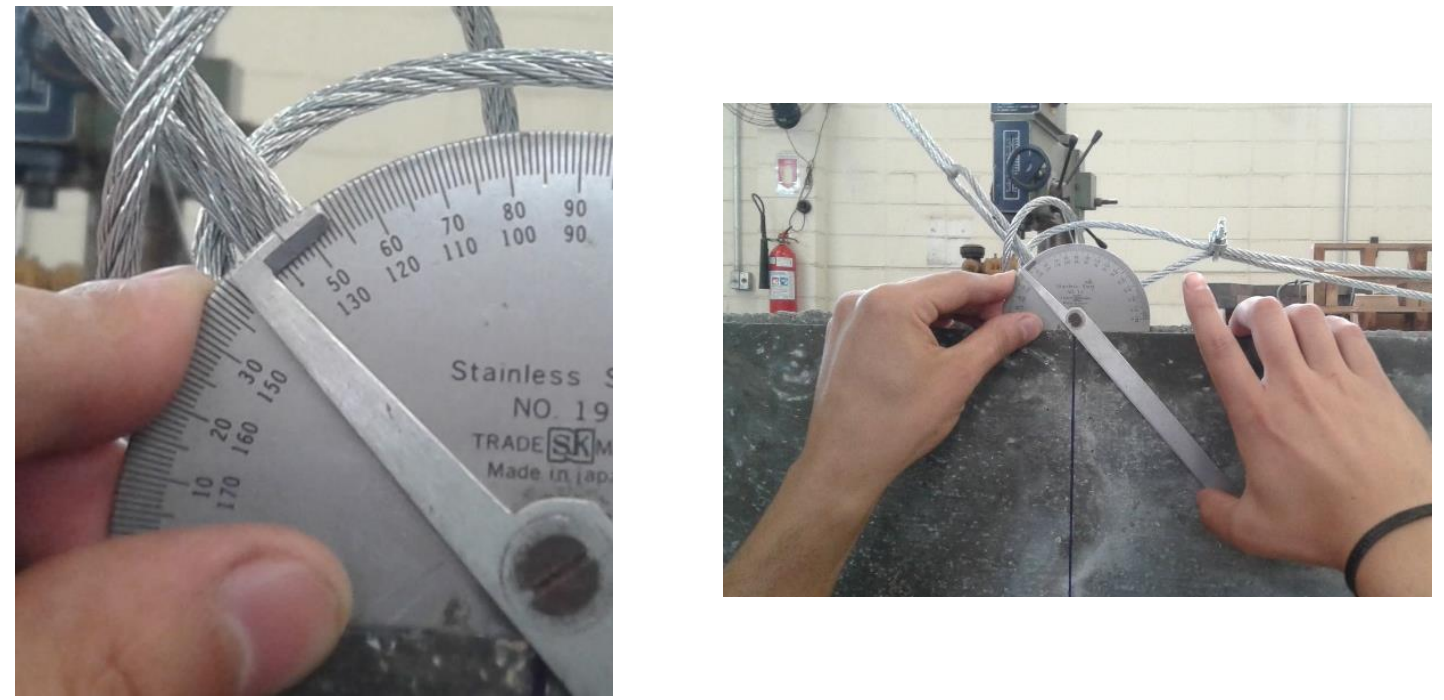

Figure 80 - Measurement of the inclination angles of the lifting cables

\section{Polar method to measure displacements and rotations}

The polar method of measurements was utilized to determine the displacements of several points of the UHPFRC beams during the test. The displacements of any point $\left(X_{Q}\right.$, $Y_{Q}, H_{Q}$ ) on the face of the beam is monitored during all test by performing measurements of vertical angles, horizontal directions, and slope distances through a measuring sensor positioned in a known point $\left(X_{P}, Y_{P}, H_{P}\right)$. The equipment is oriented from a reference point $R$, with coordinates $\left(X_{R}, Y_{R}, H_{R}\right)$. The sensor is a Robotic Total Station, model Theomat Coaxial Reflectorless Automated Total Station (TCRA1201+), with an angular precision of 1 " and linear precision of $2 \mathrm{~mm}+2 \mathrm{ppm}$. Figure 81 presents the mentioned points and the geometrical quantities for the general polar method. 


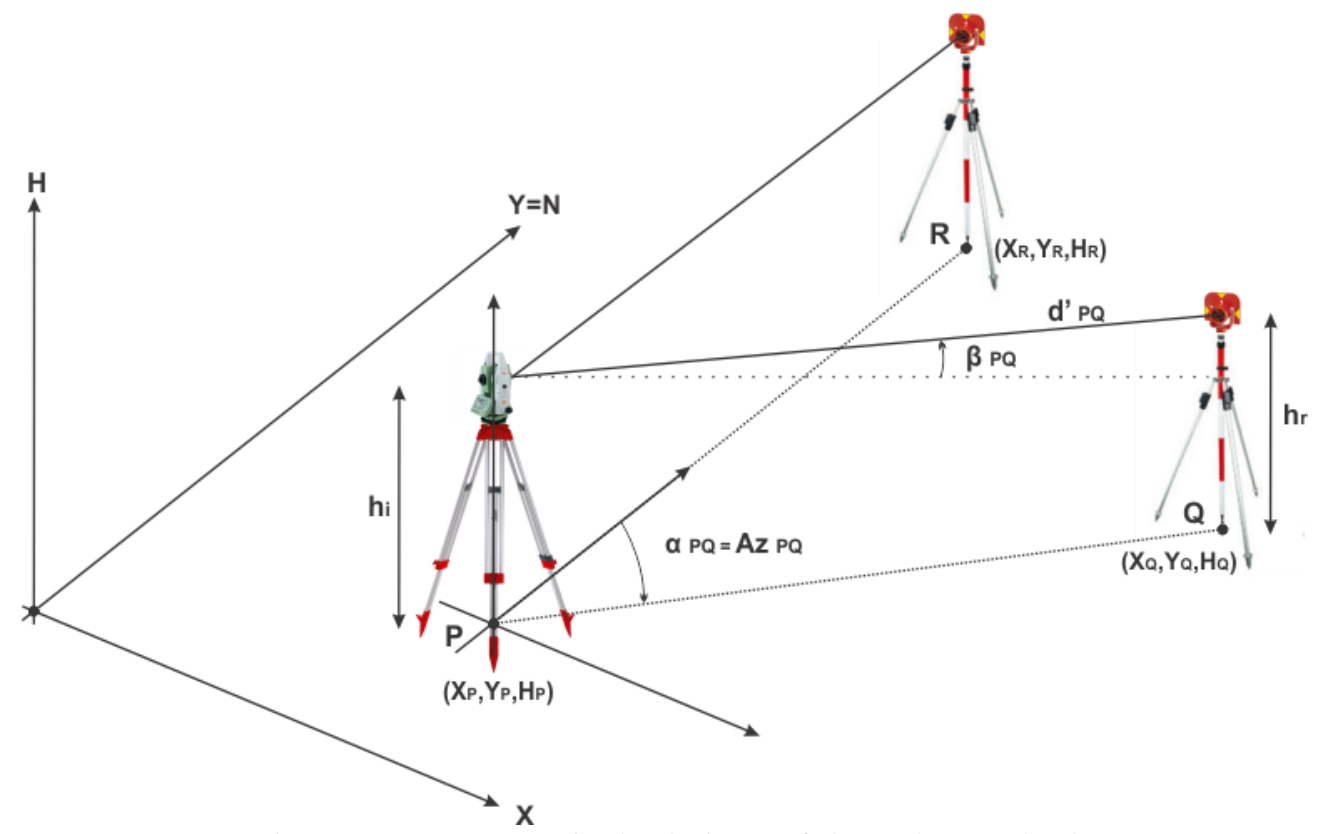

Figure 81 - Geometrical relations of the polar method

Then, the coordinates of the point $Q$ can be calculated

$$
\begin{aligned}
& X_{Q}=X_{P}+d_{P Q}^{\prime} \cos \left(\beta_{P Q}\right) \operatorname{sen}\left(A z_{P Q}\right) \\
& Y_{Q}=Y_{P}+d_{P Q}^{\prime} \cos \left(\beta_{P Q}\right) \cos \left(A z_{P Q}\right) \\
& H_{Q}=H_{P}+d_{P Q}^{\prime} \operatorname{sen}\left(\beta_{P Q}\right)+h_{i}-h_{r}
\end{aligned}
$$

Where $A z_{P Q}$ is the azimuth of alignment, $\beta_{P Q}$ is the vertical angle of height observed in point $P, d^{\prime}{ }_{P Q}$ is the inclined distance between the points $P$ and $Q, h_{i}$ and $h_{r}$ are the height of the instrument at point $P$ and height of the reflective tape at point $Q$. For the present tests, $h_{i}$ and $h_{r}$ are taken as zero, then the point $P$ coincides with the optical center, and point $Q$ coincides with the reflective tapes. The reflective tapes fixed on one face of the beam enable measurements until $100 \mathrm{~m}$. Their positions at beam 1 and beam 2 are presented in Figure 82 and Figure 83, respectively. Points $Q$ are called now as RT (reflective tape).

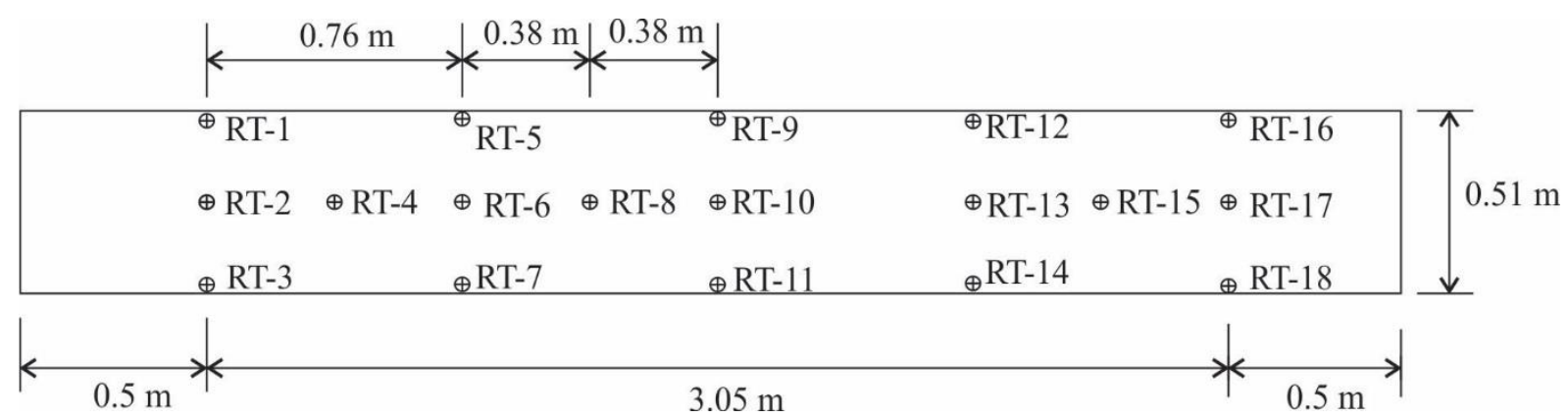

Figure 82 - Position of reflective tapes on beam 1 


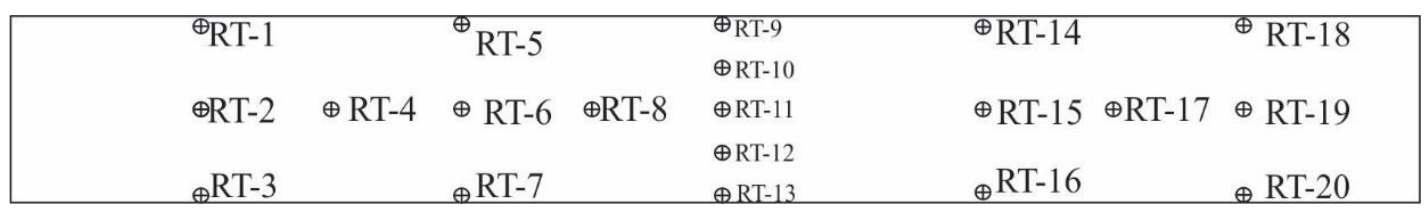

Figure 83 - Position of reflective tapes on beam 2

For the measurements, the point $P$ was fixed at the coordinate $\mathrm{X}_{\mathrm{P}}=5000.0 \mathrm{~m}, \mathrm{Y}_{\mathrm{P}}=$ $1000.0 \mathrm{~m}$, and $\mathrm{H}_{\mathrm{P}}=100.0 \mathrm{~m}$. After, the total station detected the reference point $R$ that represents the initial orientation in the horizontal direction $\left(\alpha=\mathrm{Az}=0^{\circ}\right)$. Then, each RT was monitored according to the number sequence for the initial state, with no load applied, as presented in Figure 84. Therefore, this same procedure was performed after each load increment.

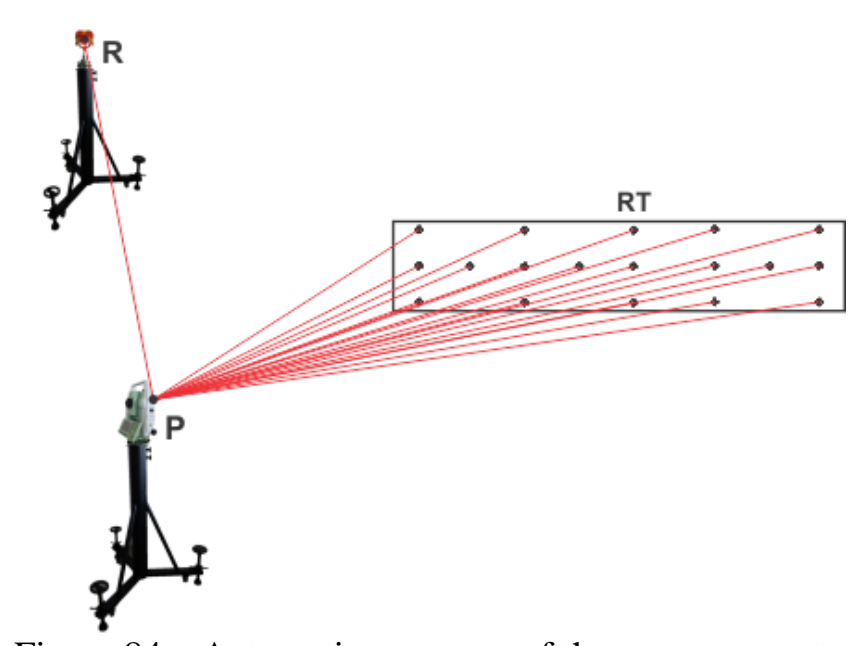

Figure 84 - Automatic sequence of the measurements

The readings made for the points RT-9, RT-10 and RT-11 in the initial step of the test for beam 1 are presented in Table 11 to exemplify the attainment of the coordinates for each step.

Table 11 - Directions and distances measured for beam 1

\begin{tabular}{cccc}
\hline Point & Az (degrees) & $\beta$ (degrees) & d' $^{\prime}(\mathrm{m})$ \\
\hline RT-9 & 53.06276 & -5.60674 & 7.0454 \\
RT-10 & 52.99979 & -7.50677 & 7.0653 \\
RT-11 & 52.90523 & -9.44415 & 7.0991 \\
\hline
\end{tabular}

This information enables to calculate the coordinates of the points with equations (50), (51) and (52), see Table 12. Also, the accuracies for each direction and the spatial accuracy are presented. 
Table 12 - Coordinates and precisions for three directions

\begin{tabular}{cccccccc}
\hline & \multicolumn{3}{c}{ Coordinates $(\mathrm{m})$} & \multicolumn{4}{c}{ Accuracy $(\mathrm{mm})$} \\
\cline { 2 - 7 } Point & $\mathrm{X}$ & $\mathrm{Y}$ & $\mathrm{Z}$ & $\sigma_{\mathrm{X}}$ & $\sigma_{\mathrm{Y}}$ & $\sigma_{\mathrm{H}}$ & $\sigma_{\mathrm{XYZ}}$ \\
\hline RT-9 & 5005.604 & 1004.214 & 99.312 & 0.8 & 0.6 & 0.1 & 1.005 \\
RT-10 & 5005.594 & 1004.216 & 99.077 & 0.8 & 0.6 & 0.1 & 1.005 \\
RT-11 & 5005.586 & 1004.224 & 98.835 & 0.8 & 0.6 & 0.2 & 1.020 \\
\hline
\end{tabular}

\section{Loading protocol}

Before the peak, the test was controlled by applying load increments of $1 \mathrm{kN}$, at quasi-static velocity, to avoid dynamical effects. After each increment, the test was stopped, and the measurements with total station were performed. When the peak was reached the load started to decrease, therefore, the control passed to be made in displacements with increments of $0.05 \mathrm{~mm}$ measured by the LVDTs. Again, the test was stopped at each increment for the measurements with the total station. The test was finished when the higher recorded displacement at the LVDTs was $0.8 \mathrm{~mm}$, corresponding to a strain at midspan of $0.4 \%$ approximately. 


\section{TEST RESULTS}

\subsection{Monotonic tensile behavior}

Ultra-high performance concrete (UHPC) that has no fibers showed a linear elastic stress-strain relationship until failure. Average modulus of elasticity was $34.6 \mathrm{GPa}$ while tensile strength was 4.4 MPa. The standard deviations were, respectively, 3.5 GPa and 0.9 MPa. The first phase of the stress-strain curve of UHPFRC was also linear elastic. In this case, the elastic modulus was higher, $39.4 \mathrm{GPa}$ and $44.5 \mathrm{GPa}$ for $1 \%$ and $2 \%$ of fibers, respectively, with standard deviations of $9.9 \mathrm{GPa}$ and $5.2 \mathrm{GPa}$. The maximum stress in this phase (cracking stress) was also higher than obtained for UHPC. The cracking stress of UHPFRC was of 4.9 MPa and 6.3 MPa and standard deviations of $0.9 \mathrm{MPa}$ and 1.0 $\mathrm{MPa}$, respectively, for $1 \%$ and $2 \%$ of fibers. So a slight improvement of elastic modulus and cracking strength was obtained with the increase of the volumetric ratio of fibers.

Figure 85 shows the stress-strain curves (envelopes of cyclic tests) of all UHPFRC specimens. It is possible to notice a stress drop just after cracking phase started. This stress drop was more pronounced in samples with $1 \%$ of fibers but also occurred with $2 \%$ of fibers. In both cases, stress recovered gradually, and UHPFRC behaved as a strain hardening material during a specific part of the multiple cracking phase. The average peak stresses of 6.1 $\mathrm{MPa}$ and 7.2 $\mathrm{MPa}$ were reached with $1 \%$ and $2 \%$ of fibers, significantly higher than peak strength of UHPC and also well above cracking stress in UHPFRC. The standard deviations were 1.2 $\mathrm{MPa}$ and 1.1 MPa, respectively. This peak stresses were reached at strains of 0.0032 and 0.0014 for $1 \%$ and $2 \%$ of fibers, respectively, well above the cracking strain that occurred around 0.00013 . This effect was possible due to fiber bridging between cracks. For peak strain, the coefficient of variation is presented that were, respectively, 0.66 and 0.79 . These values mean that the standard deviations are of the order of the average showing the high dispersion of this variable.

However, at some stage of the hardening process, a macrocrack was formed that started the softening phase. The cracking stages are well described in recent references (BENZERZOUR et al., 2017) and (WILLE; KIM; NAAMAN, 2011). A probable cause for the arising of a macrocrack is the interconnection of the formed microcracks due to the continued increase in strain. Then, the crack opening due to fiber pullout in this macrocrack governed the behavior in this phase. Even though strain localization occurred, UHPFRC 
could still maintain a gradual drop of stress for both fiber contents until a considerable strain around 0.055 . Consequently, the toughness of UHPFRC was of $0.155 \mathrm{MPa}$ and $0.165 \mathrm{MPa}$ with $1 \%$ and $2 \%$ of fibers, respectively. Toughness was determined as the area under the stress-strain diagram. The standard deviations were, respectively, $0.002 \mathrm{MPa}$ and 0.029 $\mathrm{MPa}$. The toughness values are remarkably superior to $0.0002 \mathrm{MPa}$ obtained in UHPC (without fibers).
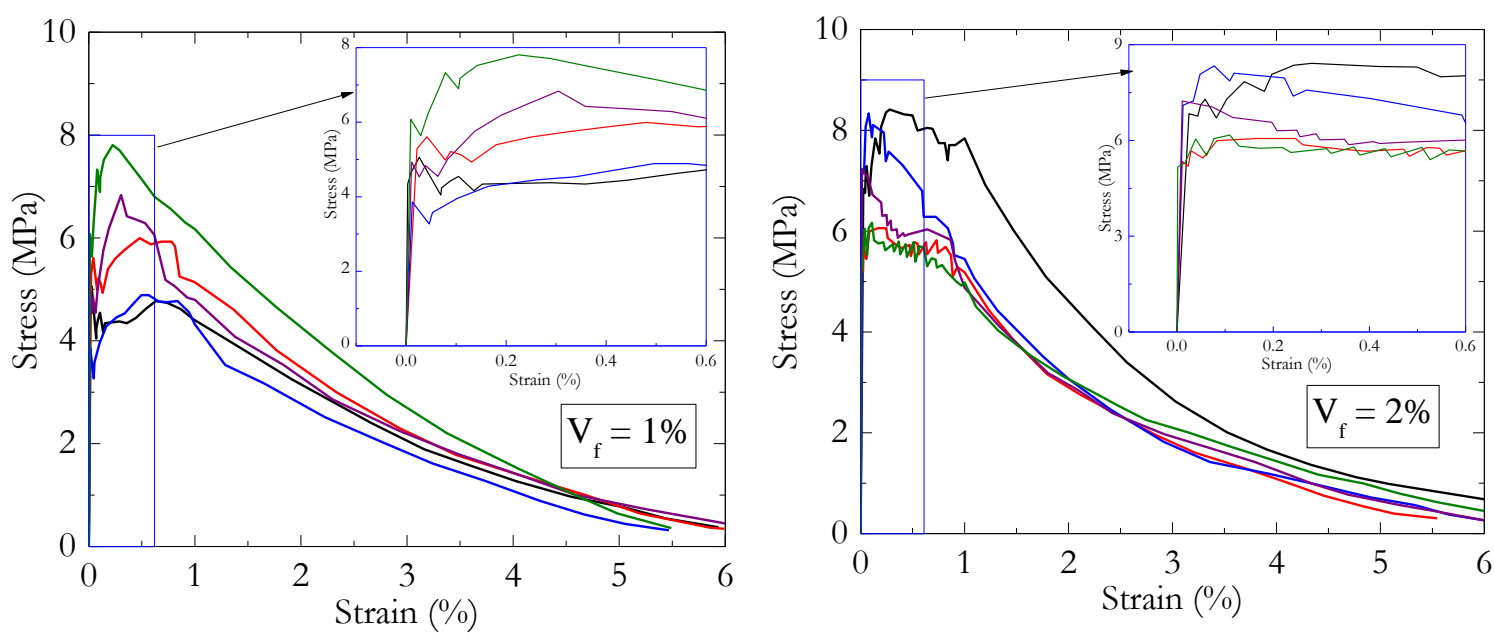

Figure 85 - Stress-strain curves (envelopes) of UHPFRC in tension

\subsection{Monotonic compressive behavior}

Under compression, UHPC behaved as a linear elastic material until failure, which was brittle. Elastic modulus was 39.6 GPa while compressive strength reached 131.5 MPa, with standard deviations of $1.6 \mathrm{GPa}$ and $8.58 \mathrm{MPa}$, respectively. The inclusion of $1 \%$ and $2 \%$ of fibers increased the elastic modulus to 40.7 and $43.4 \mathrm{GPa}$, respectively. The standard deviations were, respectively, 2.3 GPa and $0.9 \mathrm{GPa}$. Compressive strength also increased to 145.2 $\mathrm{MPa}$ and $150.5 \mathrm{MPa}$, respectively, with $1 \%$ and $2 \%$ of fibers. The standard deviations were, respectively, 3.85 $\mathrm{MPa}$ and $6.67 \mathrm{MPa}$. A slight increase in strain at peak stress was noticed from 0.0035 in UHPC to 0.0039 in UHPFRC with $1 \%$ of fibers and 0.0040 with $2 \%$ of fibers. The coefficients of variation were, respectively, 0.057, 0.089 and 0.067. However, more pronounced was the increase in toughness from $0.24 \mathrm{MPa}$ in UHPC to $0.89 \mathrm{MPa}$ in UHPFRC (1\% of fibers) and 1.11 MPa in UHPFRC (2\% of fibers). The standard deviations were, respectively, $0.02 \mathrm{MPa}, 0.13 \mathrm{MPa}$, and $0.13 \mathrm{MPa}$. This increase in toughness is mainly due to the post-peak behavior of UHPFRC in the stress-strain curve under compression (see Figure 86) that presented a much more gradual drop of stress. Besides this, the significant residual stress that UHPFRC maintained at a high level of strain (0.015) contributed to the 
high toughness observed. Residual stress for UHPFRC with $1 \%$ of fibers was $36.4 \mathrm{MPa}$ while UHPFRC with $2 \%$ of fibers showed the residual stress of $50.2 \mathrm{MPa}$. The standard deviations were, respectively, 9.75 MPa and 9.84 MPa.
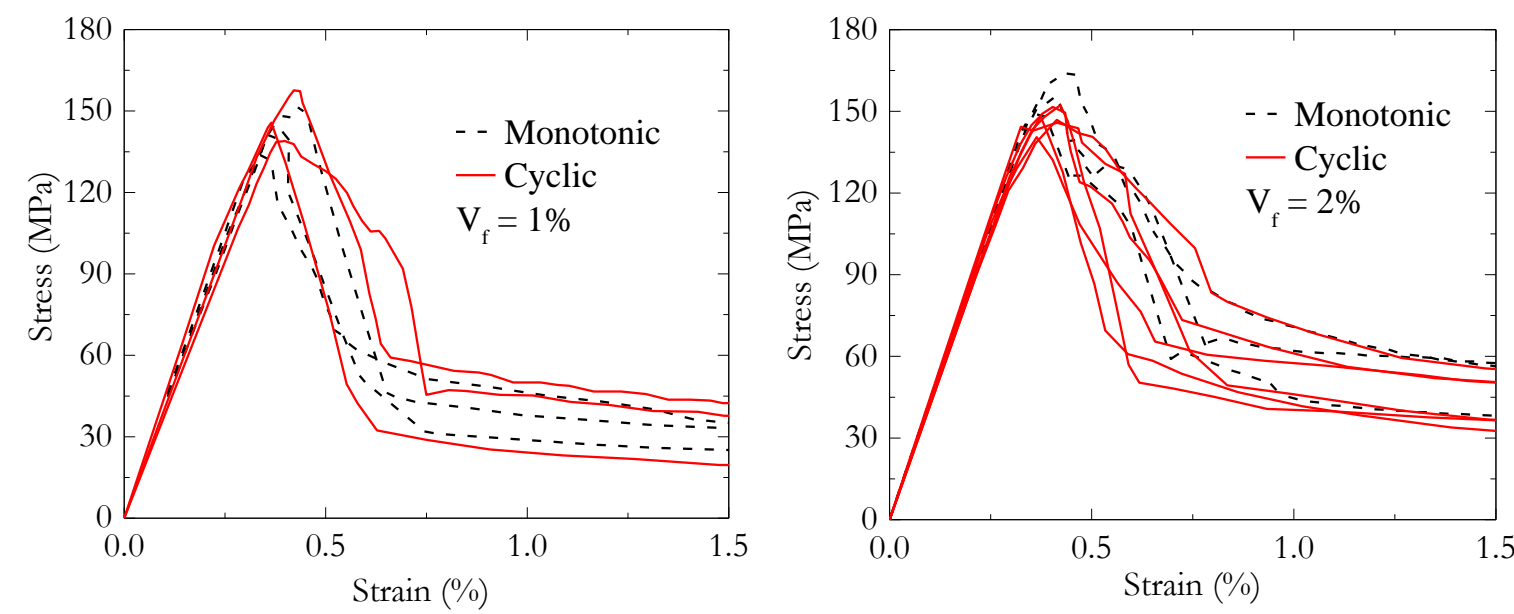

Figure 86 - Stress-strain curves of UHPFRC in compression

As discussed in topic 6.1, an experimental study was developed to determine Batch 2. Figure 87 shows the evolution of the compressive strength and modulus of elasticity with age. The results are for UHPC. During the period between 28 and 35, the material was heat treated at $70^{\circ} \mathrm{C}$.
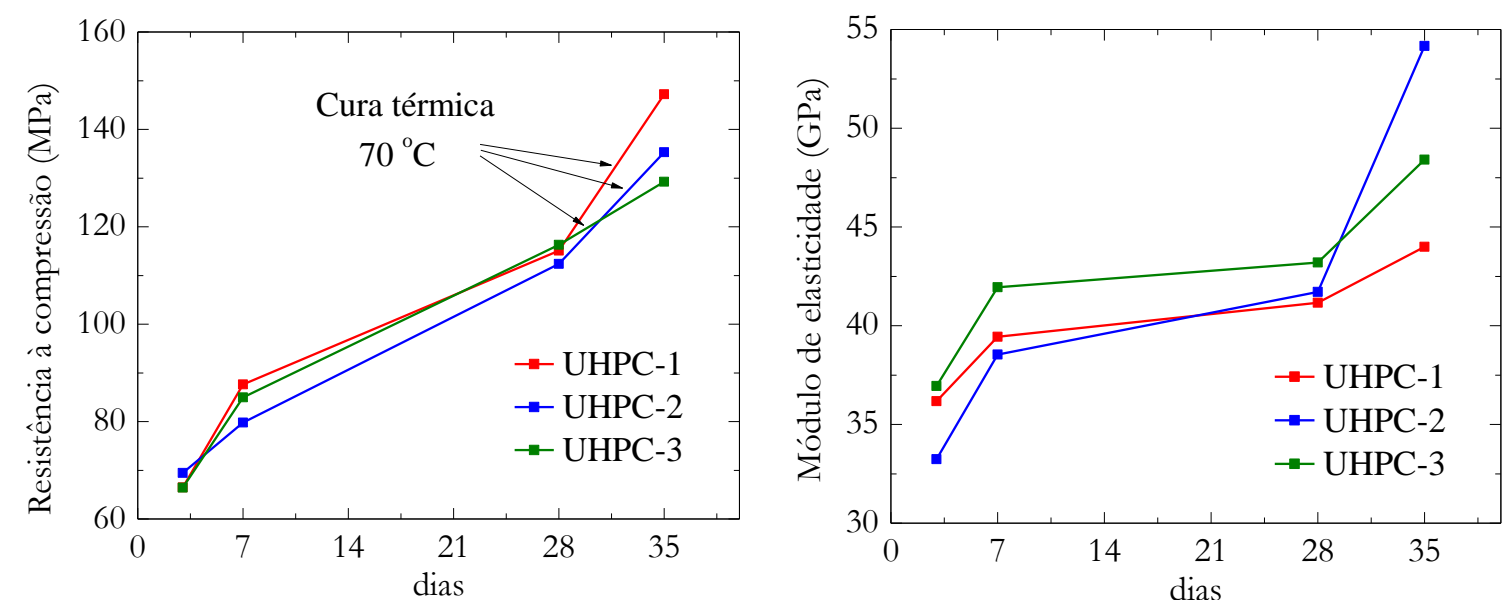

Figure 87 - Compressive strength evolution of UHPC

The mixes considered were UHPC-1 (1:1.1:0.25:0.3), UHPC-2 (1:1:0.25:0.3), and UHPC-3 (1:1.1:0.25:0.5). The proportions are in sequence for cement, sand, silica fume and quartz powder. The sequence presented is cement, fine sand, silica fume and quartz powder. From Figure 87, at 28 days the difference between the average compressive strength was $1.04 \%$ between mixes UHPC-1 and UHPC-3. The modulus of elasticity was higher for UHPC-3, mixed with more quartz powder. The difference for the UHPC-1 was $4.7 \%$ at 28 days. 
It can be noticed that the higher volume of sand for the UHPC-1 resulted in resistance $2.4 \%$ higher when comparing the results at 28 days of UHPC-1 and UHPC-2 from Figure 87. The difference in the modulus of elasticity was $1.34 \%$.

The heat treatment had a significant effect on the modulus of elasticity of the UHPC2, which increased by $29.8 \%$. The compressive strength increased by $20.4 \%$. Figure 88 presents the uniaxial compressive strength for the three batches at 3,28 and 35 days.
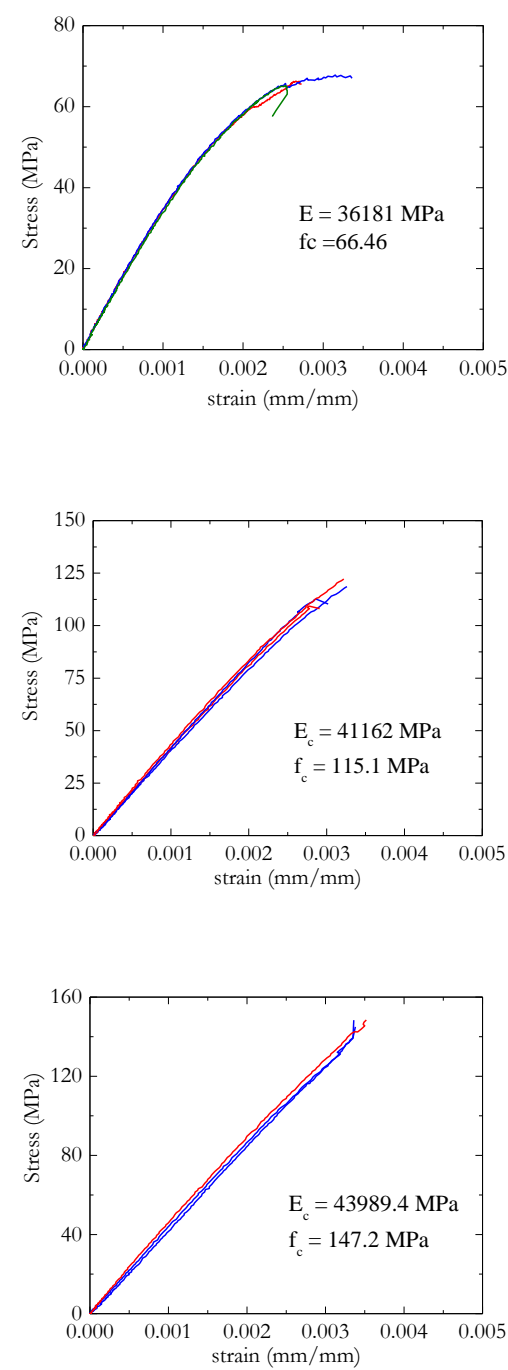

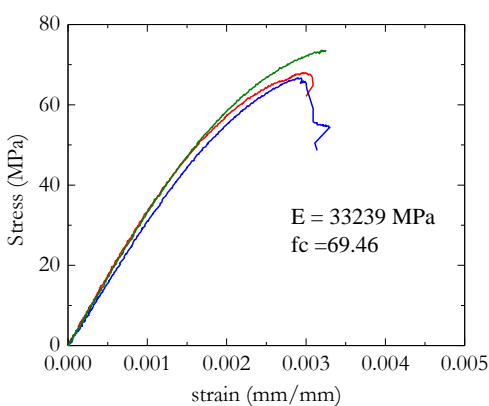

(a)

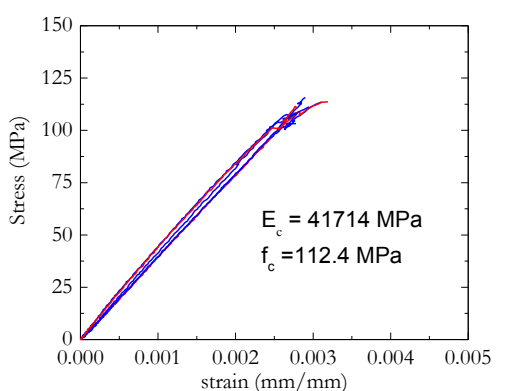

(b)

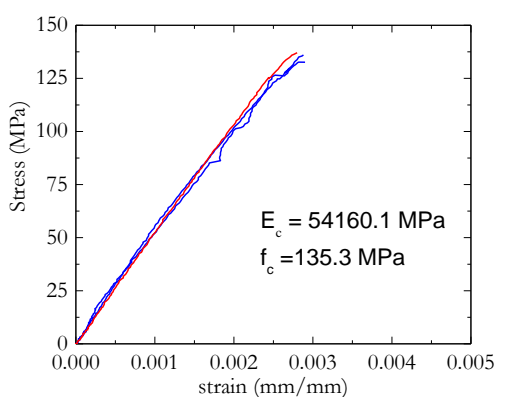

(c)
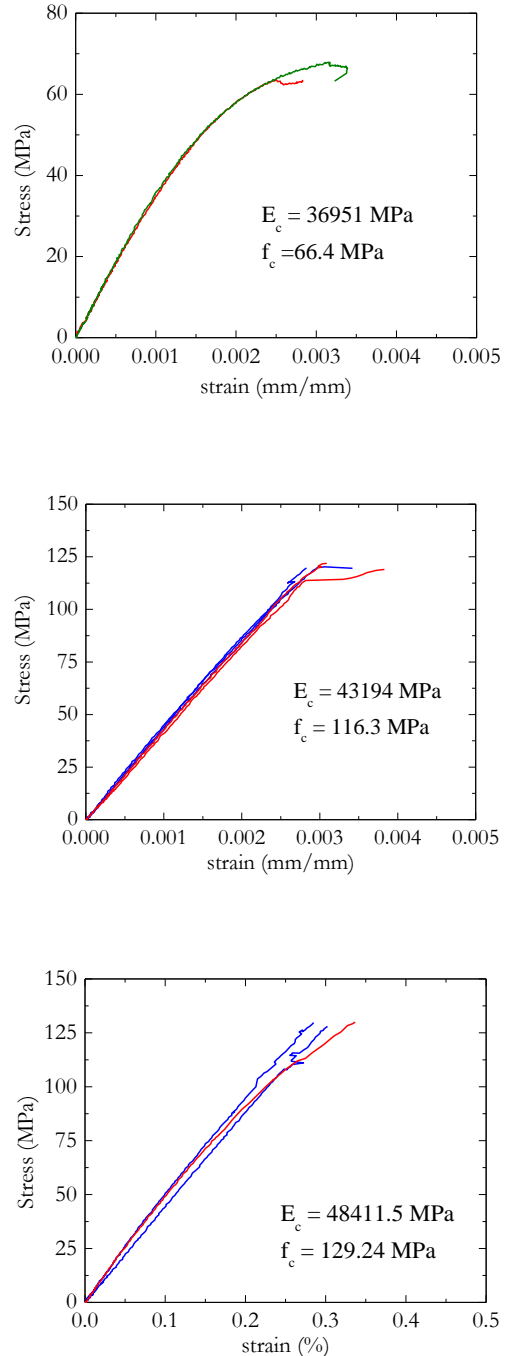

Figure 88 - Compressive response of mixes UHPC-1, UHPC-2 and UHPC-3 at (a) 3 days, (b) 28 days and (c) 35 days, respectively

The mix UHPC-3 was chosen for Batch 2. From the production of the UHPFRC beams to be tested, samples were extracted to evaluate the material behavior. Figure 89 presents the results of compressive behavior for UHPFRC with $2 \%$ of fibers at 28 days without heat curing and 35 days with heat curing. These last tests were performed only to evaluate the material behavior because the beams were not heat treated. 


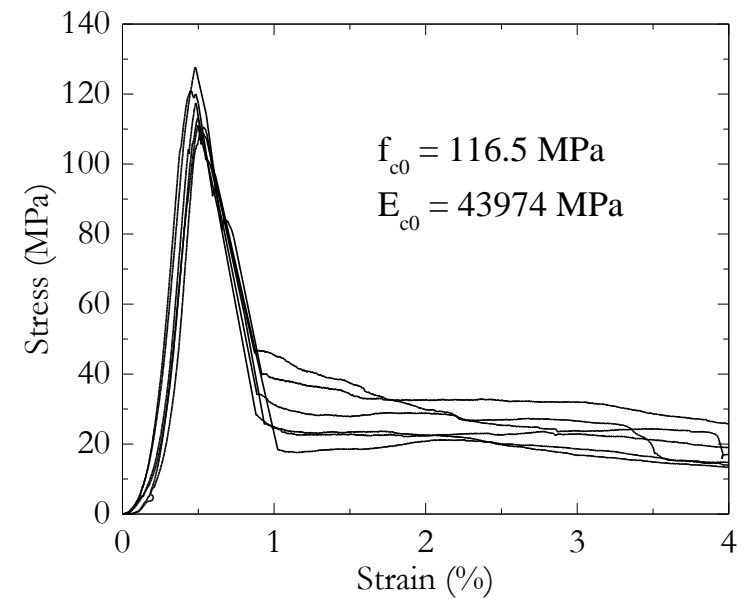

(a)

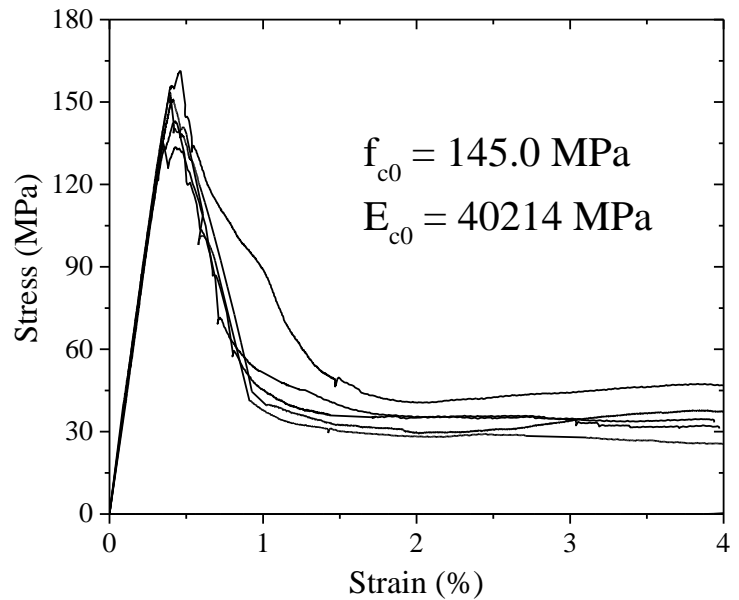

(b)

Figure 89 - Compressive response of UHPFRC with $2 \%$ of fibers (a) without heat curing and (b) with heat curing

The addition of fibers had a small effect on the compressive strength and modulus of elasticity of UHPFRC without heat curing, by comparing the results of UHPC-3 at Figure 88 (b) and UHPFRC in Figure 89 (a). However, the results of heat treated UHPC-3, at Figure 88 (c), and UHPFRC, at Figure 89 (a), showed an increase of $10.9 \%$ in compressive strength and a decrease in the modulus of elasticity of $20.4 \%$. These observations require more investigation and are out of the scope of the present research.

Internal cracking of fiber reinforced concrete under compression is difficult to investigate. The cylindrical samples were cut in half longitudinally to show the crack pattern. Figure 90 shows the cut section and the load direction.
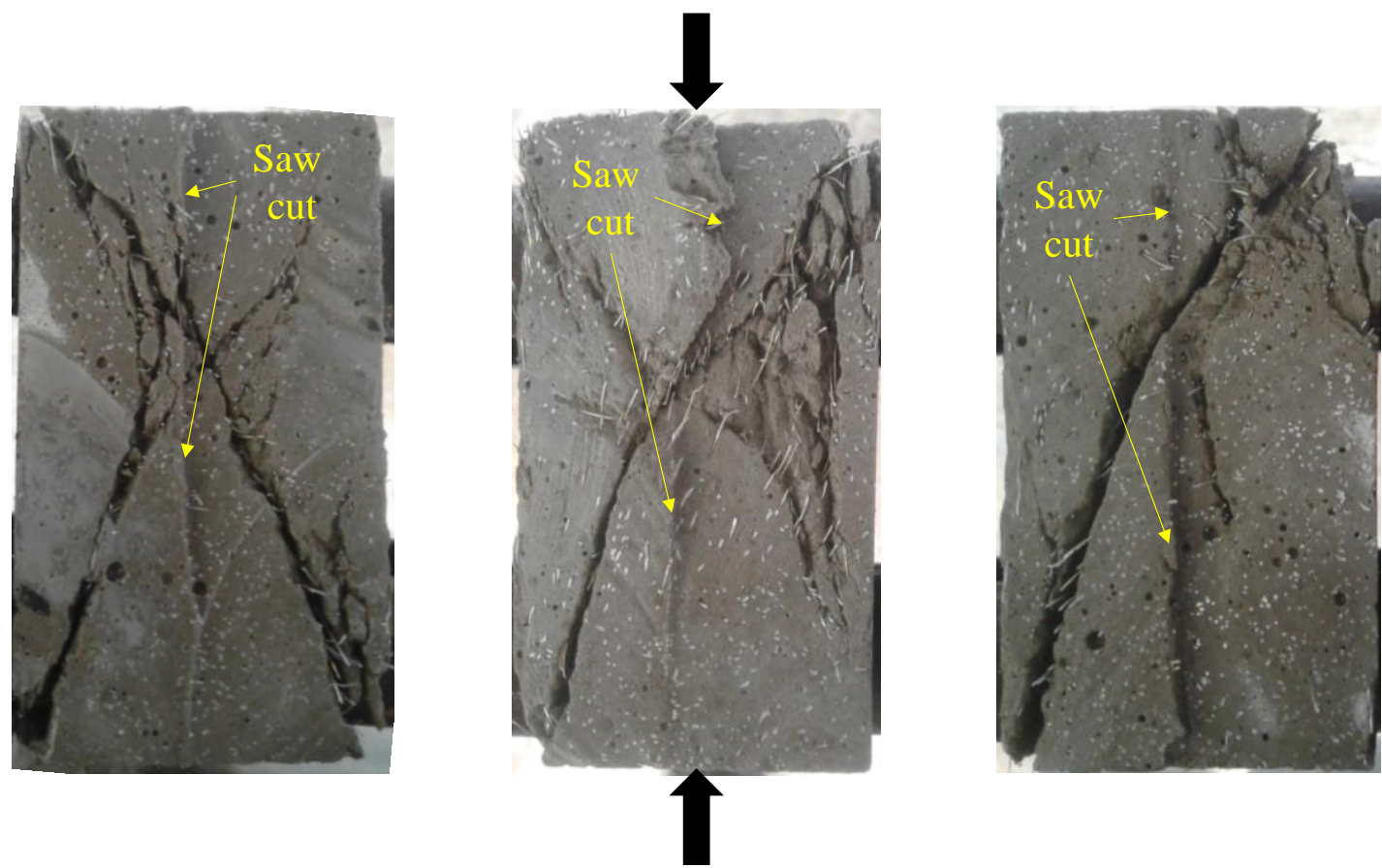

Figure 90 - Internal cracking pattern of the compression samples 
Inclined cracks were the main pattern, some forming cones with base in the top and bottom faces of the cylinder and others with a main inclined crack. The mechanism that leads to this differences in the crack pattern was not explained in literature and, also, the damage mechanisms are still based on speculation and qualitative evaluations. One hypothesis is that the crack follows weak planes, $(\mathrm{OESCH}, 2015)$, for example where most of the fibers are in the direction of the principal compressive stress. Then, due to tensile strain, cracks are formed transversally.

The fibers that cross the cracks are gradually pulled-out with increasing loading. Under compression, besides moving away, the crack faces slide relative to each other shearing the fibers that act as dowels. Figure 91 presents a fragment of UHPFRC extracted from a compressive sample that shows the fibers pulled-out in a crack face.

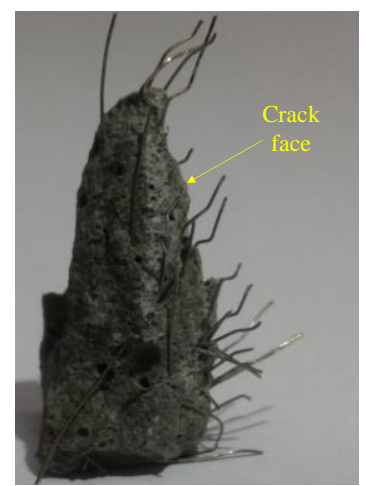

Figure 91 - Fiber pull-out under compression

Several damage mechanisms are associated with the mechanical response of the cement-based materials. The quantification of such mechanisms can be made through cyclic tests, but the evidence of the occurred mechanisms require the use of another methodology. Scanning electron microscopy (SEM) was utilized in the present research to investigate the probable mechanisms involved in the degradation of UHPFRC. However, other methods can also be applied as the acoustic emission that enables to differentiate the type of degradation mechanisms during loading, (KRAVCHUK; LANDIS, 2018), and X-ray computed tomography that enables to visualize such events at each scan, (TRAINOR et al., 2013b). These research fields are currently under development.

Figure 90 presented the macrocracks developed in the compressive cylinders. Samples with a maximum dimension of $20 \times 20 \mathrm{~mm}$ were extracted with a diamond saw to identify mechanisms of degradation. The face of the sample about the crack was not polished, only cleaned with water and air blast. Figure 92 presents a global view of the crack face of the composite matrix. 

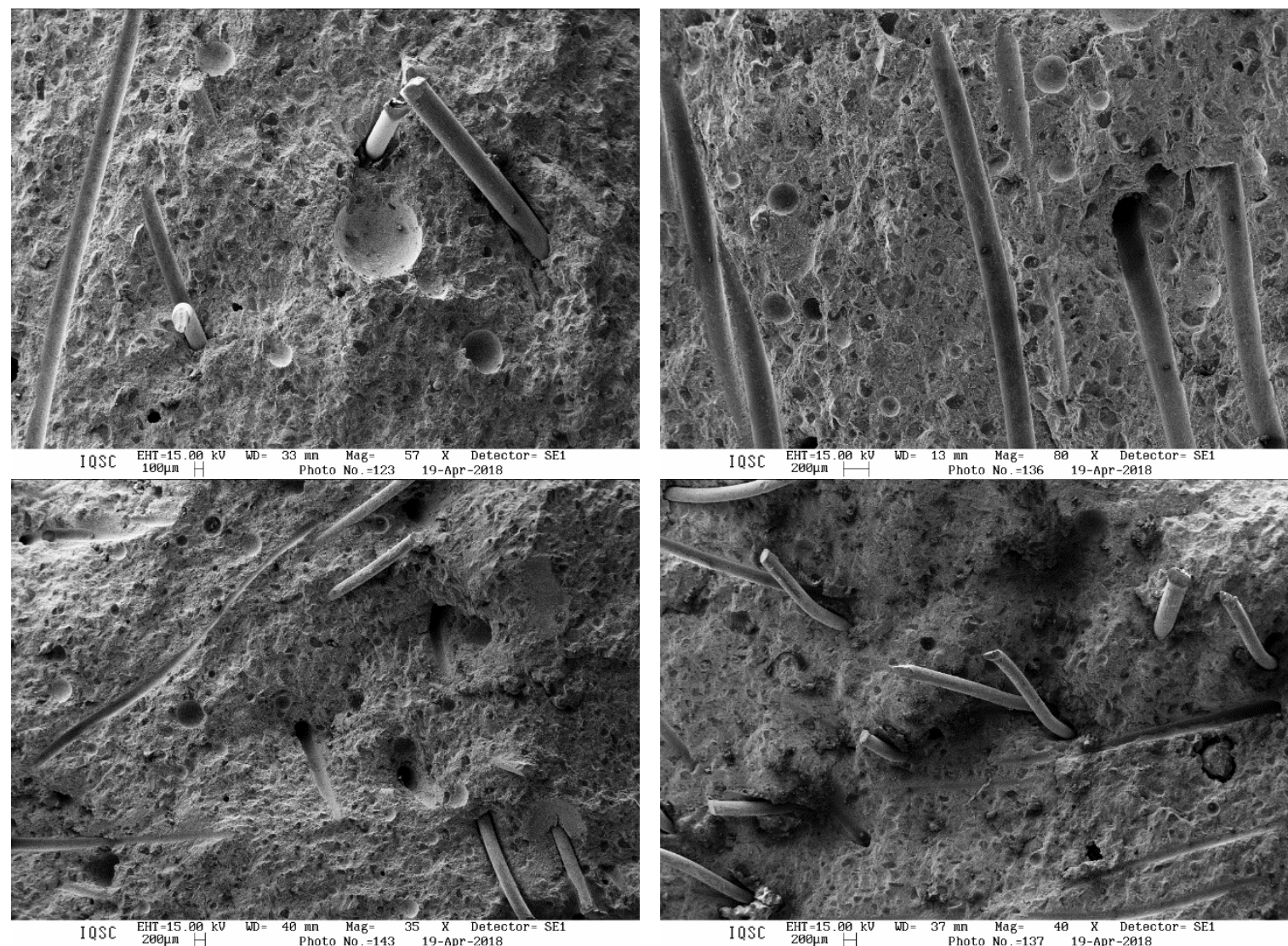

Figure 92 - SEM images of the crack face of UHPFRC after compression

It can be seen from the figures that part of the fibers was aligned to the cracks. In this fibers, only adhesion was mobilized as damage mechanism, which confers small resistance to cracking development. The damage mechanisms occurred in the interaction between transverse fibers, and the matrix are the main factors that make the composite ductile. Furthermore, due to the fibers be the greater heterogeneities of UHPFRC, the fiber/matrix interface is probably a weak zone. Thus, the cracking propagation can be influenced by some alignment of the fibers transversally to principal tensile strains in the compressed sample. Such evidence was also observed by (OESCH, 2015). Figure 93 presents some SEM images of the fiber/matrix interface for cases where debond occurred. 

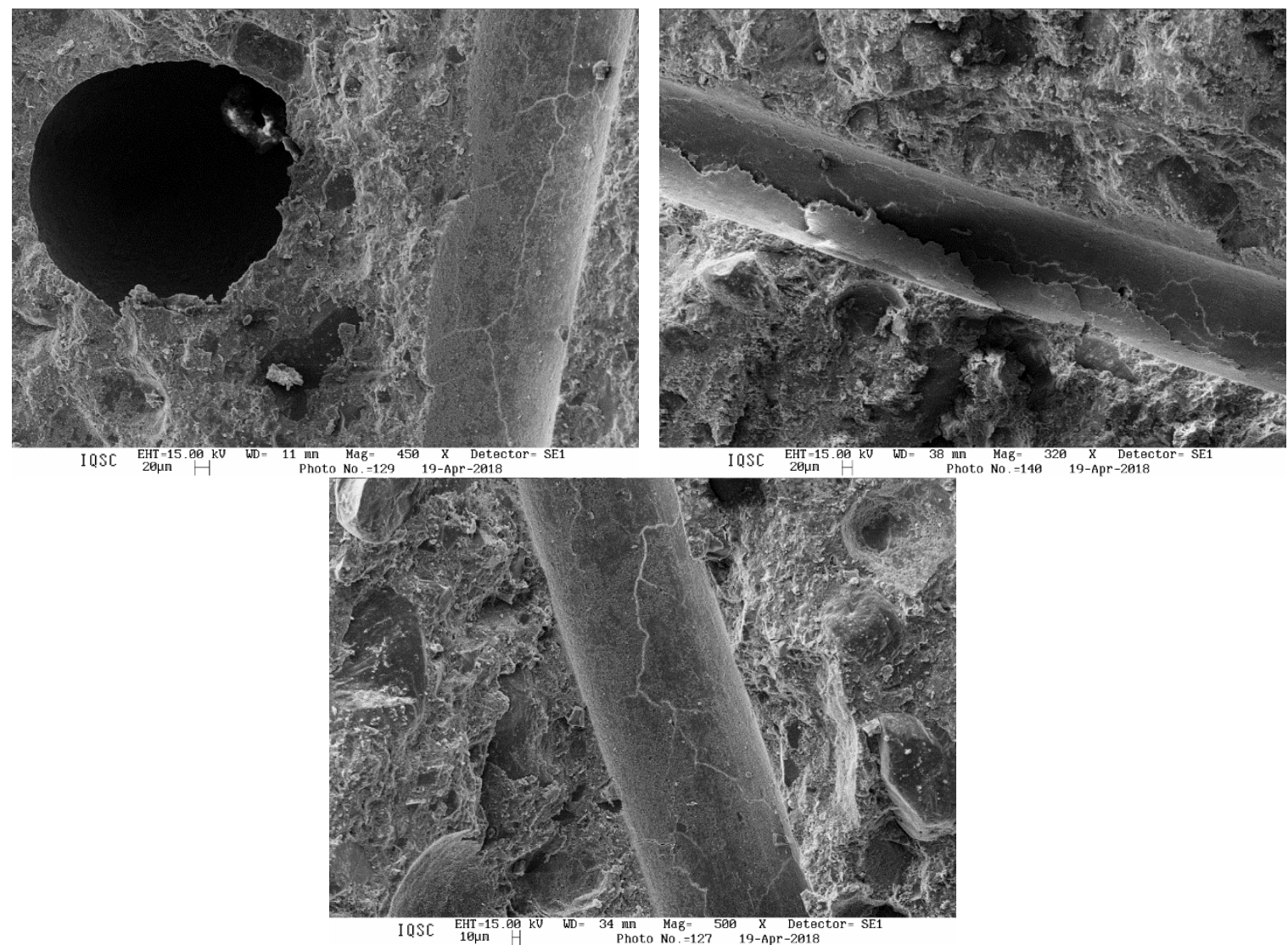

Figure 93 - Microcracks in the fiber-matrix interface

The microcracking observed in this regions were a pattern, evidencing the weak zone. The higher water to binder ratio compared to the bulk matrix can influence the early age microcracking of this regions due to autogenous shrinkage, for example. This microcracks probably interconnect through the cement matrix with pores, and the other microcracks present around the fine sand. Figure 94 presents a crack interconnection between the fiber interface and an air void.

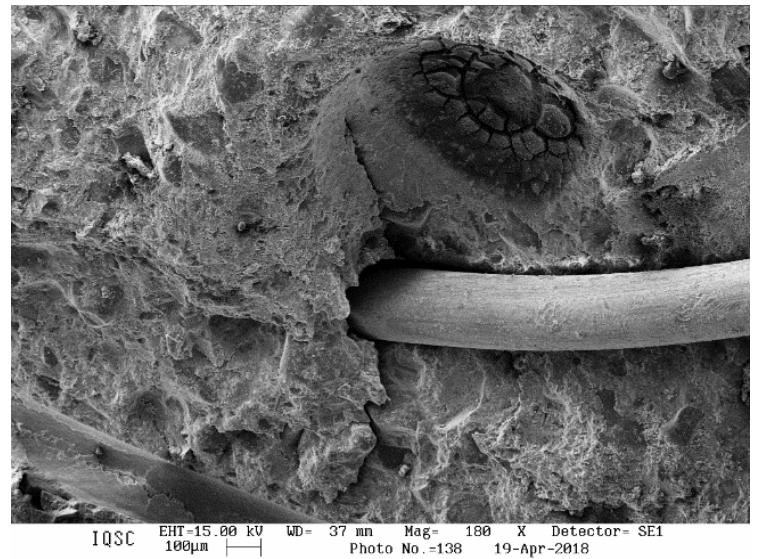

Figure 94 - Crack propagation between weak zones

This coalescence is the primary cause of the appearance of macrocracks. Besides, it can be seen from the Figure 94 that the air void is also cracked and the interface around fiber 
was degraded due to pull-out (large space between fiber and matrix). The air voids collapse was a common observation in the UHPFRC after the compressive test, see Figure 95.
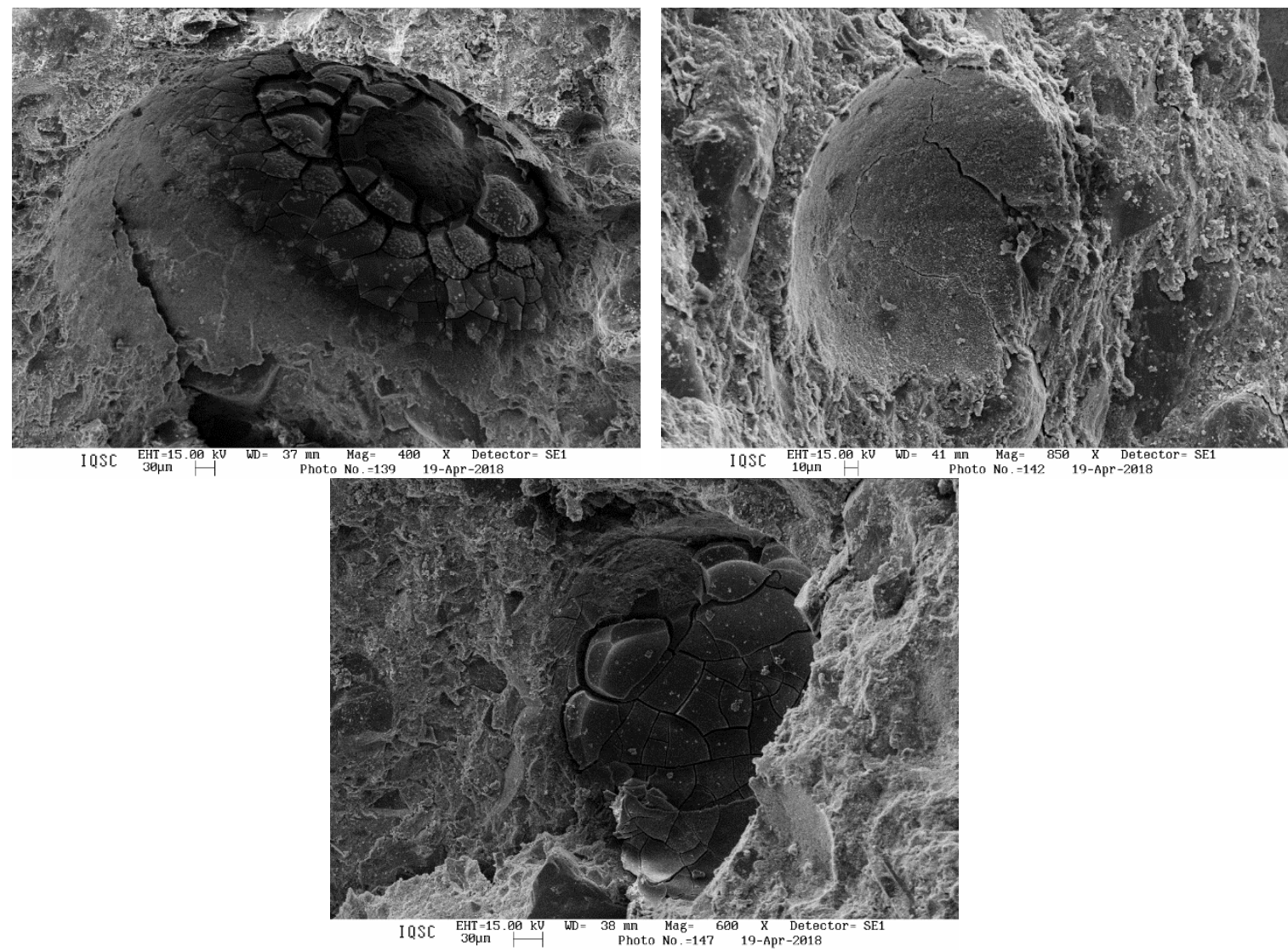

Figure 95 - Air void collapse

Discontinuities in a solid matrix are regions of stress concentration. Under compression, the tensile stress concentration occurs above and below the voids, therefore, favoring the formation of splitting cracks. After, the air void fragments due to the continuous compressive strain and the brittle nature of the material surrounding its walls.

Figure 96 presents a fiber that was not pulled out. The damage in the matrix surrounding the fiber is low.
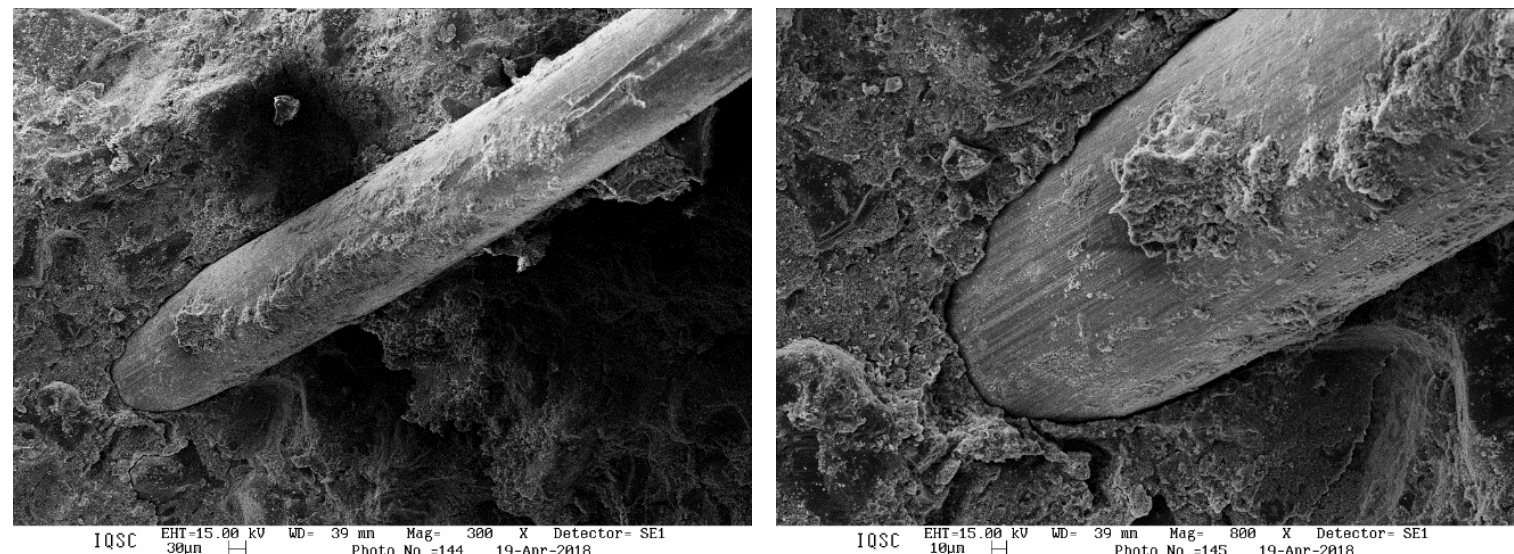

Figure 96 - Fiber with low damage with matrix 
It seems that the contribution in dissipating energy of such fiber was by debonding mechanisms. This figure also shows the excellent adhesion of the cementitious material to the fiber. Residual material is deposited on the fiber surface. Probably, this fiber did not suffer a frictional slip, which would clean its surface.

The fibers have a fundamental role in the ductility of UHPFRC. The post-peak is governed by the damage mechanisms that occur after the beginning of cracking. As is common utilize straight steel fibers in UHPFRC, the main mechanism developed in such case is the frictional slip. Figure 97 presents the opening left by a pulled fiber.

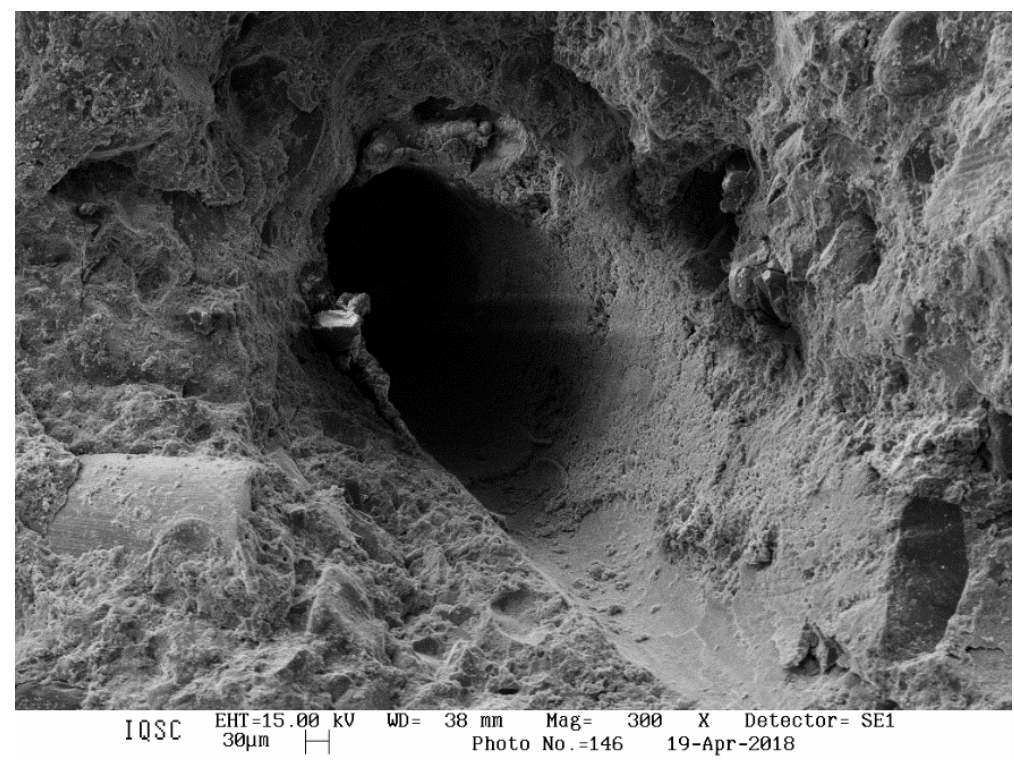

Figure 97 - Opening left due to the pull-out of a fiber

It can be seen from the figure some particulate material in the walls, which probably was disaggregated from the interface during frictional slip. This phenomenon is favored by the increased roughness of the fiber surface by depositing metals like copper.

Aggregate/matrix interfaces are also weak zones. Under compressive stresses states, the cracking in heterogeneous solids tends to deviate the particles with a higher modulus of elasticity, as the sand grains. This mechanism dissipated larger quantities than in tension stress states that are unstable. Also, above and below the sand, the porous interface is crushed during compressive loading.

Silica fume has a high surface area due to its fineness, and consequently, the particles tend to attract to each other. The formed agglomerations that did not disperse during mixing react and form low-calcium C-S-H, (DIAMOND; SAHU; THAULOW, 2004). These structures present volumetric instability and constitute further weak zones in the composite 
matrix. Figure 98 (a) to (d) presents the cracking in the compressive sample in agglomerations of silica fume. Figure 98 (b) presents a zoom of the crack in Figure 98 (a).

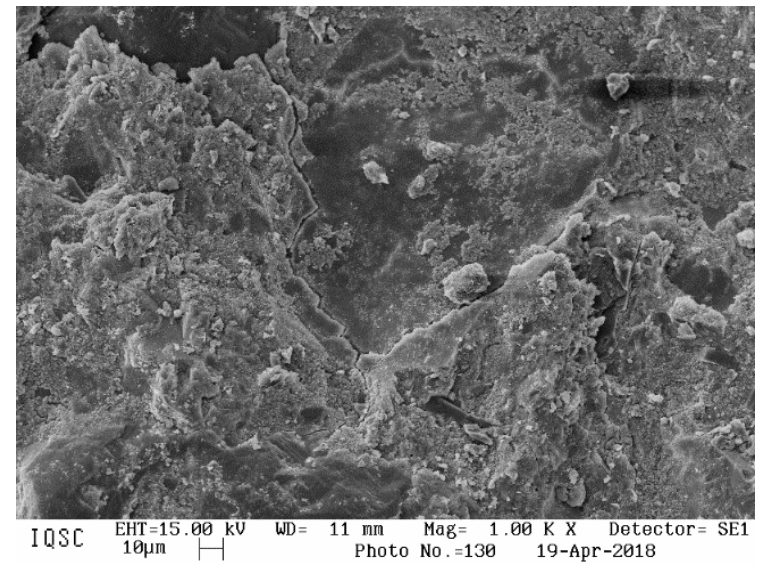

(a)

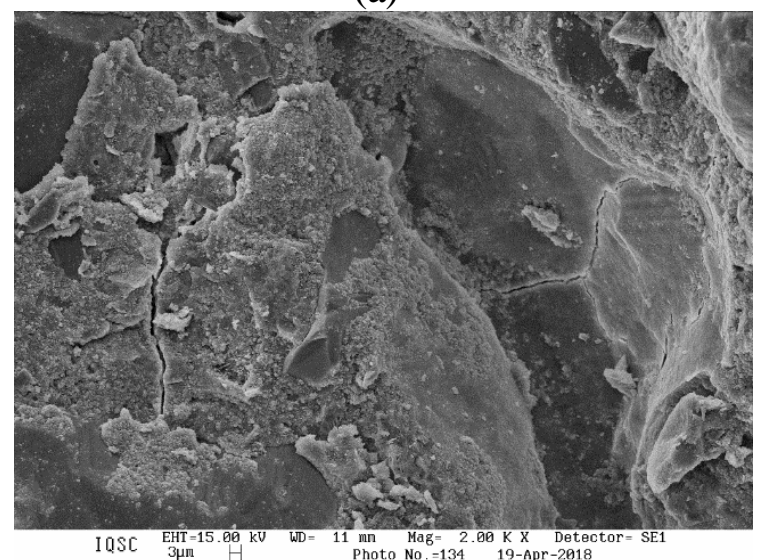

(c)

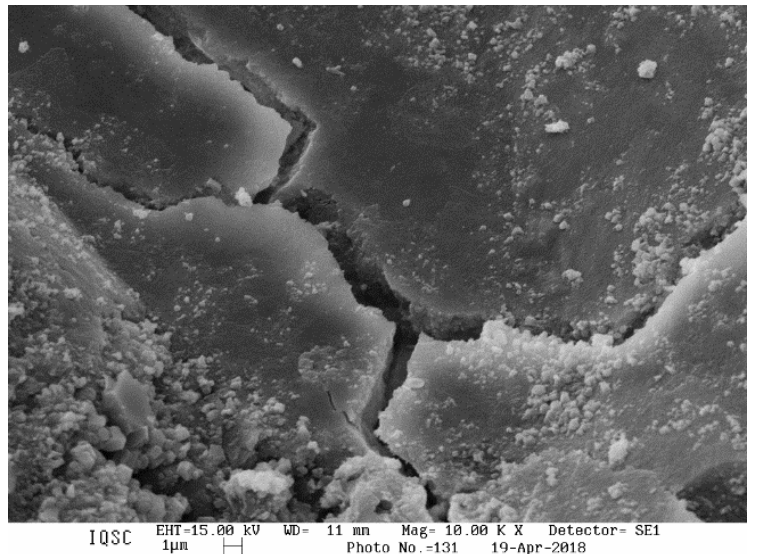

(b)

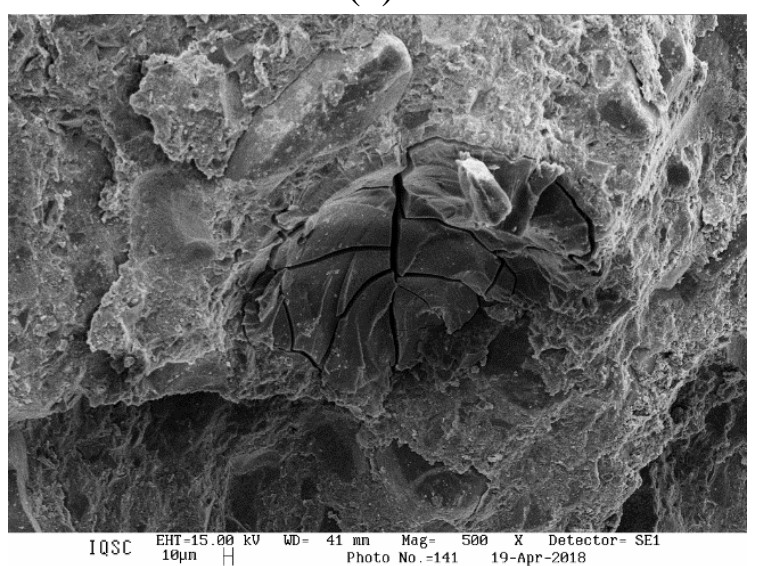

(d)

Figure 98 - Cracking on reacted agglomerations of silica fume

It can be seen from Figure 98 (a) and (c) that the smooth surfaces are cracked instead of the rough one. These homogeneous surfaces are the reacted agglomerations. (SANCHEZ; INCE, 2009) and (DIAMOND; SAHU; THAULOW, 2004) published similar finds, Figure 99.
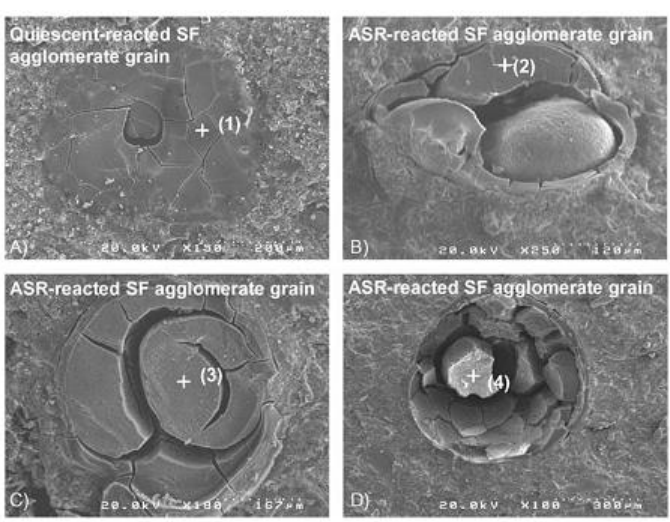

Figure 99 - Reacted agglomerations of silica fume from (SANCHEZ; INCE, 2009) and (DIAMOND; SAHU; THAULOW, 2004)

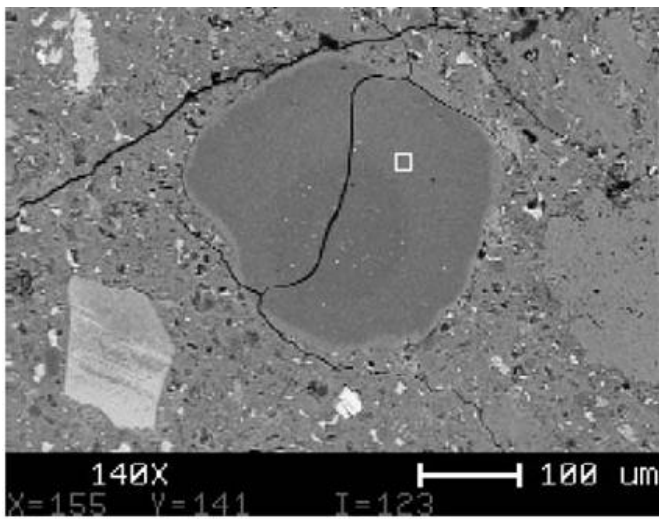




\subsection{Monotonic flexural response}

Figure 100 (a) and (b) shows the load-deflection curves respectively for UHPC and UHPFRC, respectively. Figure 100 (a) presents two results with different colors for the same mix of the composite without fibers. In the case of UHPC, failure occurred suddenly after the first crack was formed. Average peak load was of $19.0 \mathrm{kN}$, and maximum deflection was of $0.038 \mathrm{~mm}$. The first crack in UHPFRC with $1 \%$ fibers was formed at $53.7 \mathrm{kN}$ and in UHPFRC with $2 \%$ fibers at $61.6 \mathrm{kN}$, significantly higher than in UHPC. Peak load was also considerably higher in UHPFRC. Average results were, respectively, $93.1 \mathrm{kN}$ and $131.2 \mathrm{kN}$ for fiber contents of $1 \%$ and $2 \%$. The axial displacement at peak load was, respectively, 0.71 $\mathrm{mm}$ and $0.60 \mathrm{~mm}$ for $1 \%$ and $2 \%$ of fiber content. The accumulated energy (area under P. $\delta$ curves) was remarkably increased from $0.5 \mathrm{kN}$.mm in UHPC to $209.2 \mathrm{kN} . \mathrm{mm}$ and 335.2 kN.mm in UHPFRC with $1 \%$ and $2 \%$ of fibers. Comparing the accumulated energy with $1 \%$ of fibers and $2 \%$ of fibers the increase was $60.2 \%$.

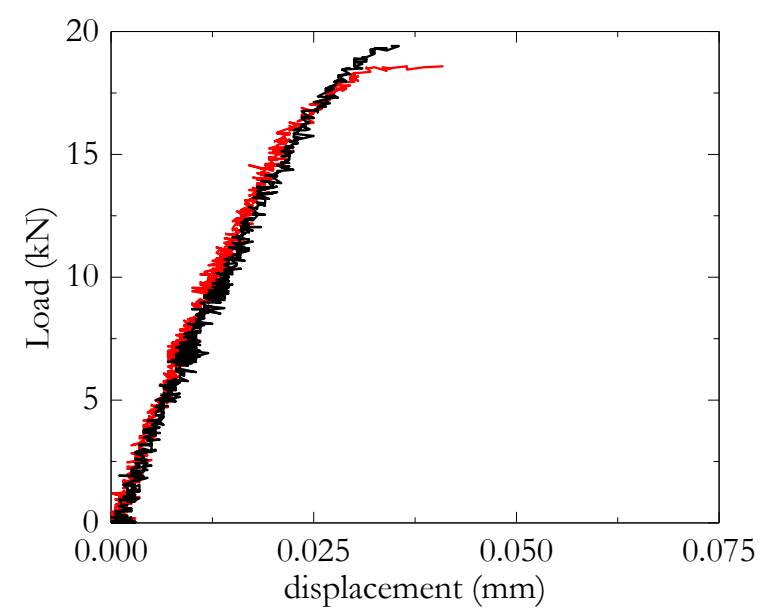

(a)

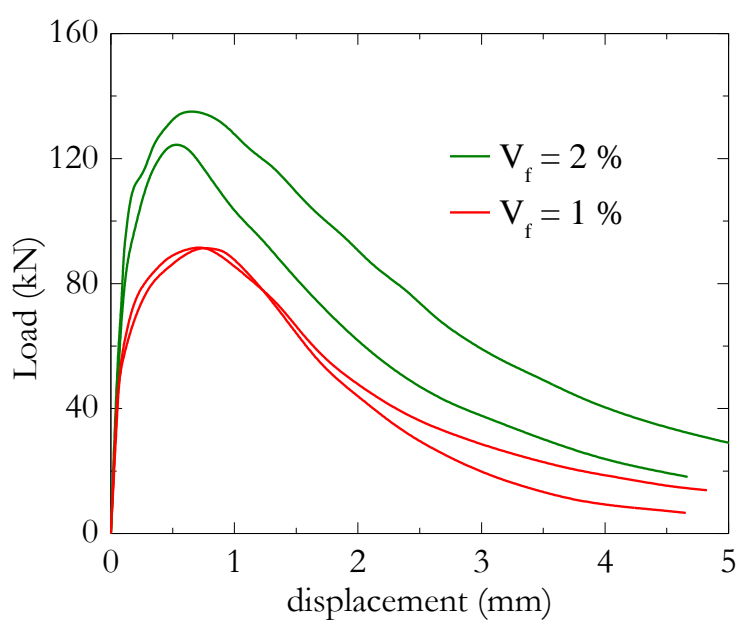

(b)

Figure 100 - Flexural tests: load-displacement curves (a) for UHPC without fiber (b) envelopes of the cyclic tests

Besides the comparison with average results, the fact that only two samples were tested for each fiber content limits the statistical significance of the presented results. However, these results are valuable to verify the applicability of the constitutive model calibrated for UHPFRC that depends on the proposed analytical equations presented in the following topics.

Normal stresses distribution can be assumed in the sections between the load application points. Thus, the cracking stress at the bottom side of the prisms can be 
determined. The parameter $f_{L O P}$, determined according to ASTM C 1609/C 1609M-05, represents this quantity

$$
f_{L O P}=\frac{P_{L O P} \times \ell}{b h^{2}}
$$

The index $L O P$ means the limit of linear proportionality. Thus, PLOP is the cracking load. $b$ and $h$ are the width, and the height of the prism and $\ell$ is the span. Table 13 presents the comparison between cracking stresses obtained from direct tensile tests and the fourpoint bending tests. Also, the results of (REN et al., 2018) for the same flexural tests a presented.

Table 13 - Comparison of cracking stress from tensile and flexural tests

\begin{tabular}{cccccc}
\hline & \multirow{2}{*}{ UHPC } & \multicolumn{4}{c}{ UHPFRC } \\
\cline { 3 - 6 } & & $1 \%$ & Increase $(\%)$ & $2 \%$ & Increase $(\%)$ \\
\hline$f_{t}(\mathrm{MPa})$ - present research & 4.4 & 4.9 & 11.4 & 6.3 & 43.2 \\
$f_{L O P}(\mathrm{MPa})-$ present research & 2.5 & 7.2 & 188.0 & 8.2 & 228.0 \\
\hline Difference $(\%)$ & $43.2 \downarrow$ & $46.9 \uparrow$ & - & $30.2 \uparrow$ & - \\
\hline$f_{t}-($ REN et al., 2018) & 6.5 & 8.4 & 29.2 & 9.0 & 38.5 \\
$f_{L O P}-($ REN et al., 2018) & 12.7 & 14.3 & 12.6 & 13.1 & 3.1 \\
\hline Difference $(\%)$ & $95.4 \uparrow$ & $70.2 \uparrow$ & - & $45.5 \uparrow$ & - \\
\hline
\end{tabular}

Similar tendencies could be observed in the table, except the increase in $f_{L O P}$ due to fiber addition of the present research. It seems that the $f_{L O P}$ for UHPC was below expected, also because was smaller than $f_{t}$ from the direct tensile test. The results of (REN et al., 2018) presented $f_{L O P}$ always greater than $f_{t}$.

Furthermore, four prisms were cast to characterize the tensile behavior of Batch 2 that was produced with $2 \%$ of fibers at the same time of the beams cast for instability tests. Figure 101 presents the results.

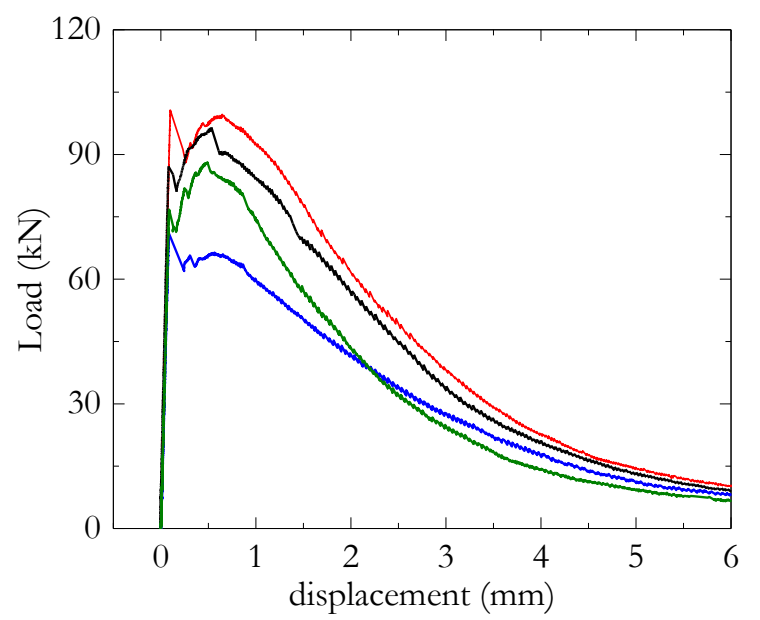

Figure 101 - Results of the four-point bending tests 
A slip-hardening behavior was observed. After the first crack, a drop in load occurred, and due to the presence of sufficient fiber content, a hardening behavior was developed. Comparing the results of Figure 100 (b) to Figure 101, the main difference is the slip after the first crack. Thus, besides the better packing of the mix for Batch 2, by addition of quartz powder, the heat treatment seems to have a higher influence in the fiber/matrix interaction. The heat-treated samples presented a hardening behavior with a slight slip after cracking. For comparison with batch 1 , the $f_{L O P}$ of the batch 2 samples was also calculated. The average $P_{L O P}$ was $84.0 \mathrm{kN}$ that was $36.8 \%$ higher than the $P_{L O P}$ resulted for the UHPFRC with $2 \%$ of fibers of the batch 1 . The $f_{L O P}$ for the batch 2 was $11.2 \mathrm{MPa}$.

Figure 102 presents the crack pattern for the non-heat treated samples of batch 2 of UHPFRC.
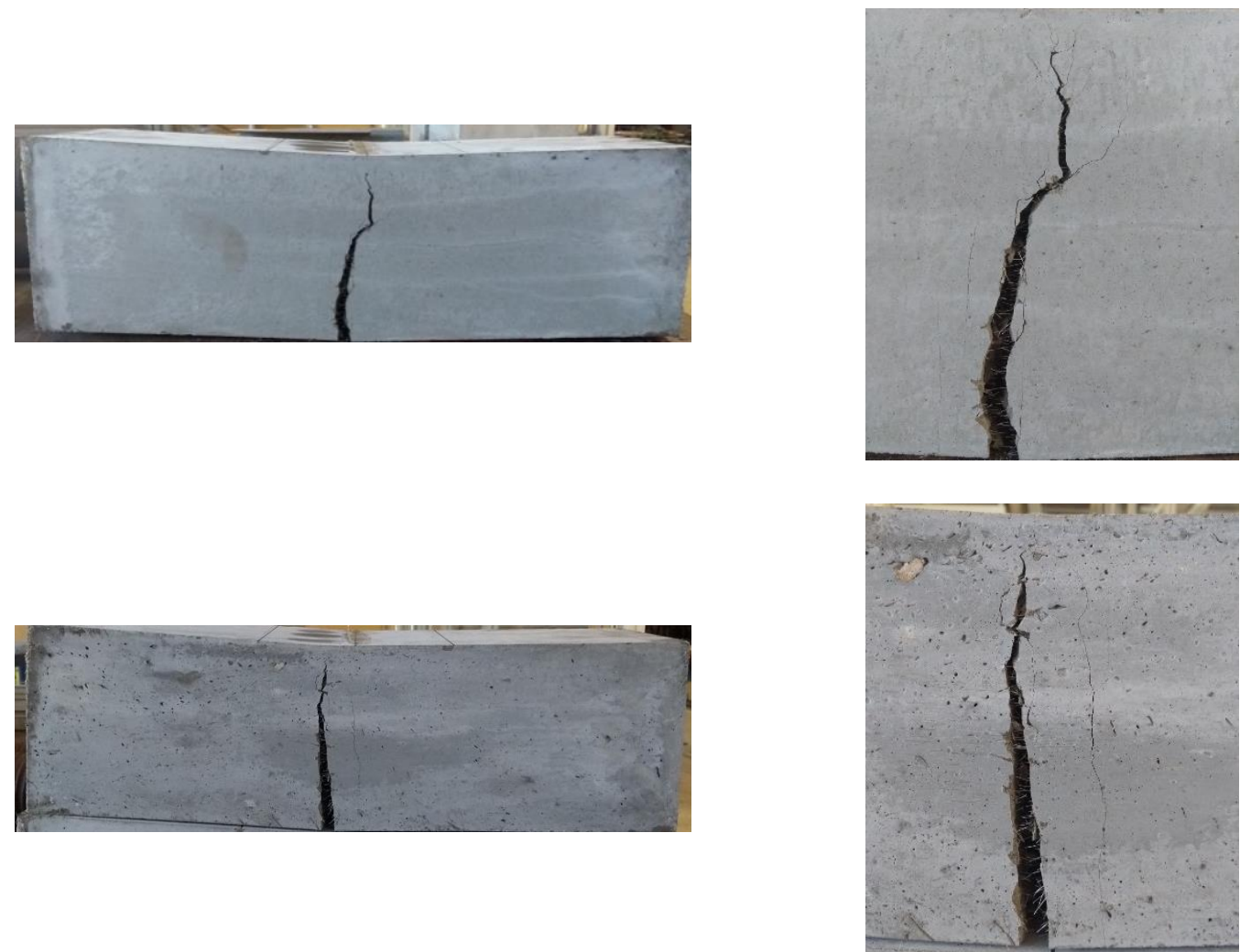

Figure 102 - Cracking pattern of the prisms tested under four-point bending test

The development of distributed cracking, as in the prisms in the figure, is a reflection of the strain hardening behavior. To further understand the UHPFRC behavior under flexure, samples with maximum dimensions of $20 \times 20 \mathrm{~mm}$ were extracted from fractured faces of the flexural prisms to perform scanning electron microscopy (SEM). The crack face was not polished and only cleaned with air bland and water. Figure 103 presents a view of four crack faces extracted from the tested flexural prisms of Figure 102. 

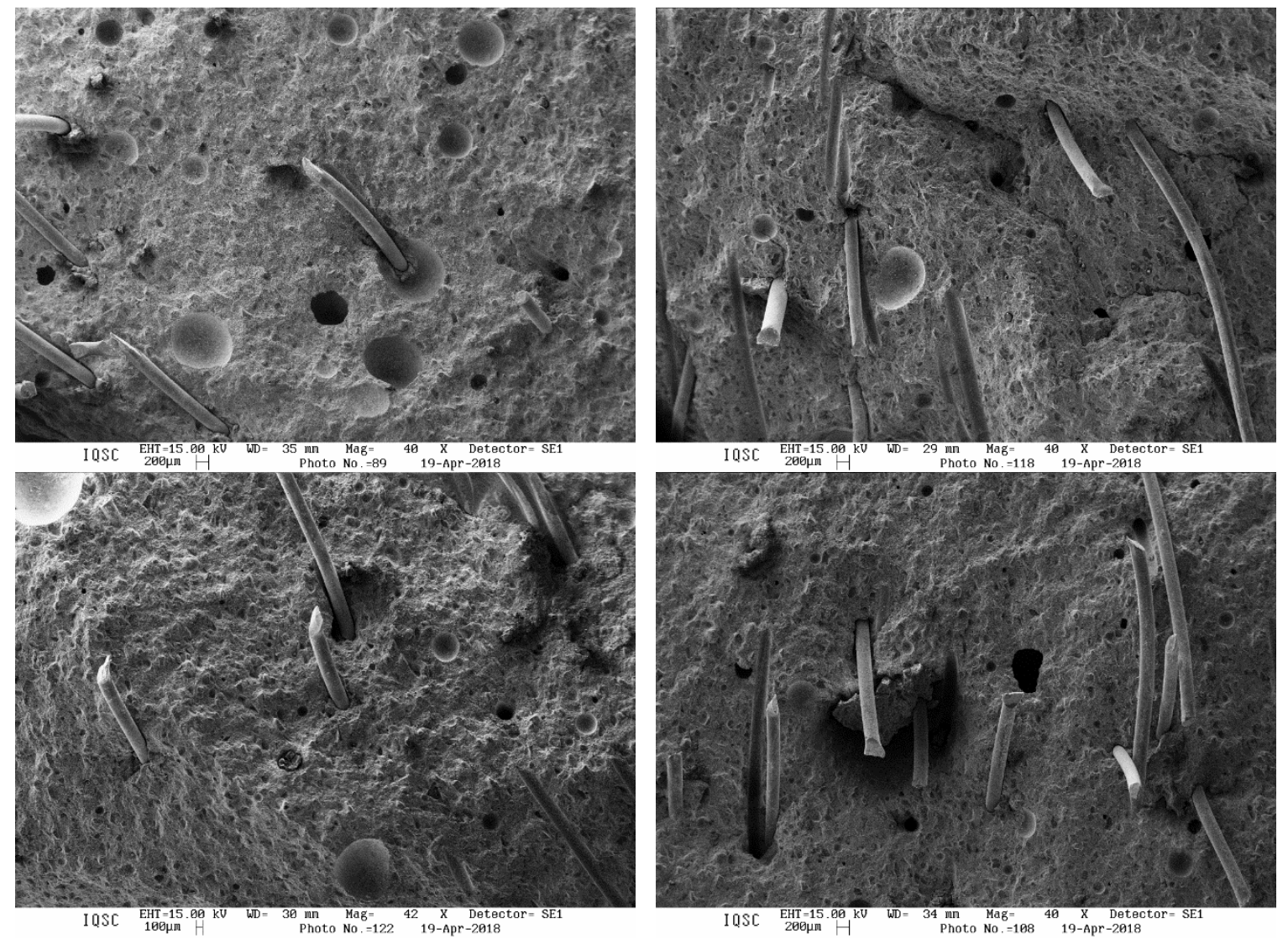

Figure 103 - Global view of the crack faces

The SEM images show the presence of pulled fibers, pores, air voids and marks of aligned fibers that were debonded from the matrix during cracking. It can be expected that a critical combination of such heterogeneities governs the formation of a macrocrack. As discussed in the previous topic, fibers and aggregates present a porous interface with the matrix. Air voids and pores are regions of stress concentration which favor the crack propagation. Also, agglomerations of reacted silica fume form weak areas.

Microcracks exist before the material carry any load. Regions in the interfacial transition zone (ITZ) that have higher water/binder ratio compared to the bulk matrix tends to present early age microcracking due to drying and thermal shrinkage. Figure 104 presents microcracking detected in the fiber/matrix interface and in an air void, which was a tendency observed in the samples. Furthermore, a zoom was made in the cracks that give its width magnitude. 

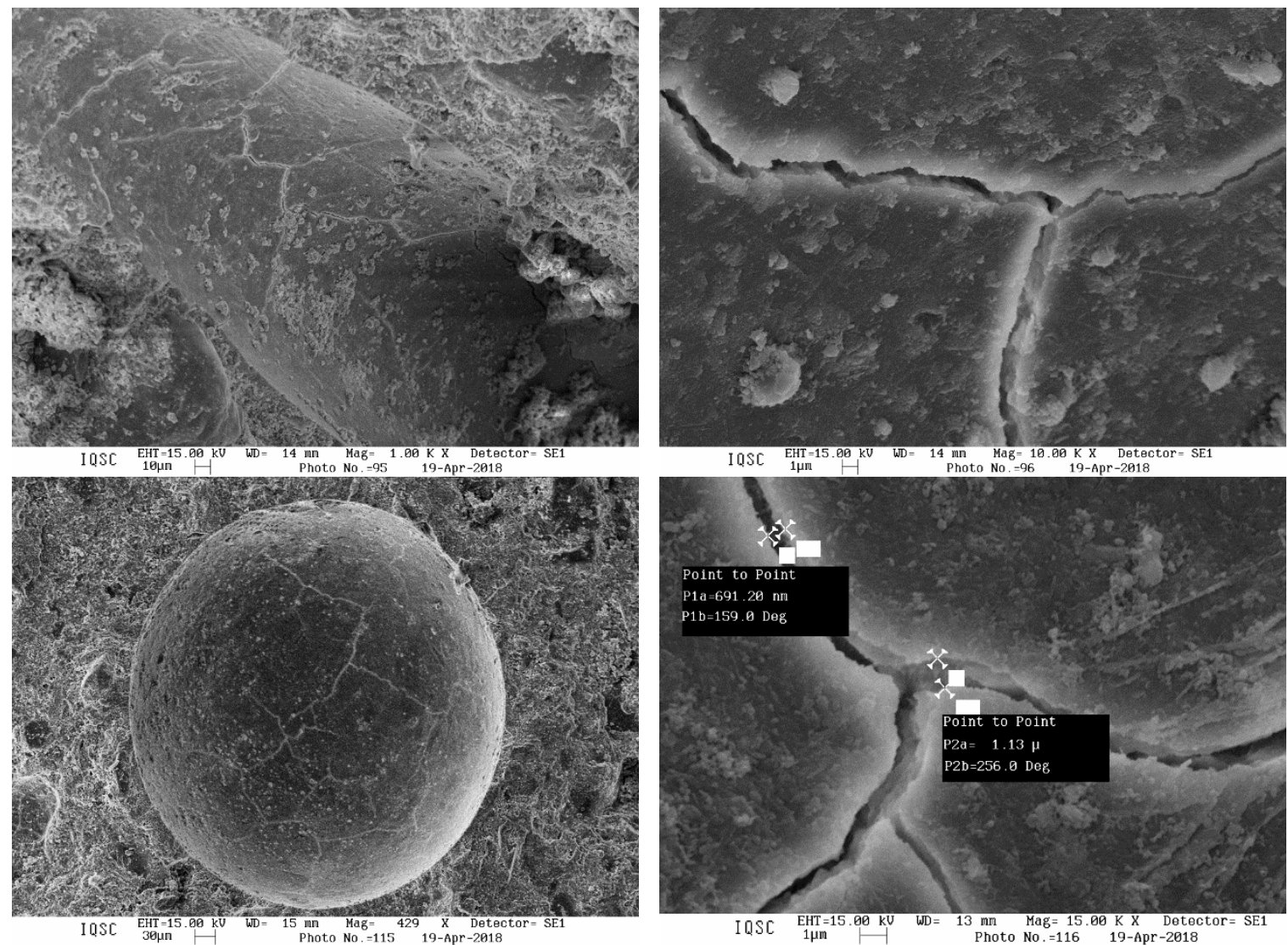

Figure 104 - Microcracks in the air voids and the fiber/matrix interface

Two measures were taken in the cracks of the air void whose width were $691.2 \mathrm{~nm}$ and $1.13 \mu \mathrm{m}$. The interconnection of such microcracks during loading causes the macrocrack appearance. Figure 105 presents some evidence of such phenomenon for which the crack coalescence was between air void and fiber/matrix interface, two fiber/matrix interfaces and two sand grain interfaces. 

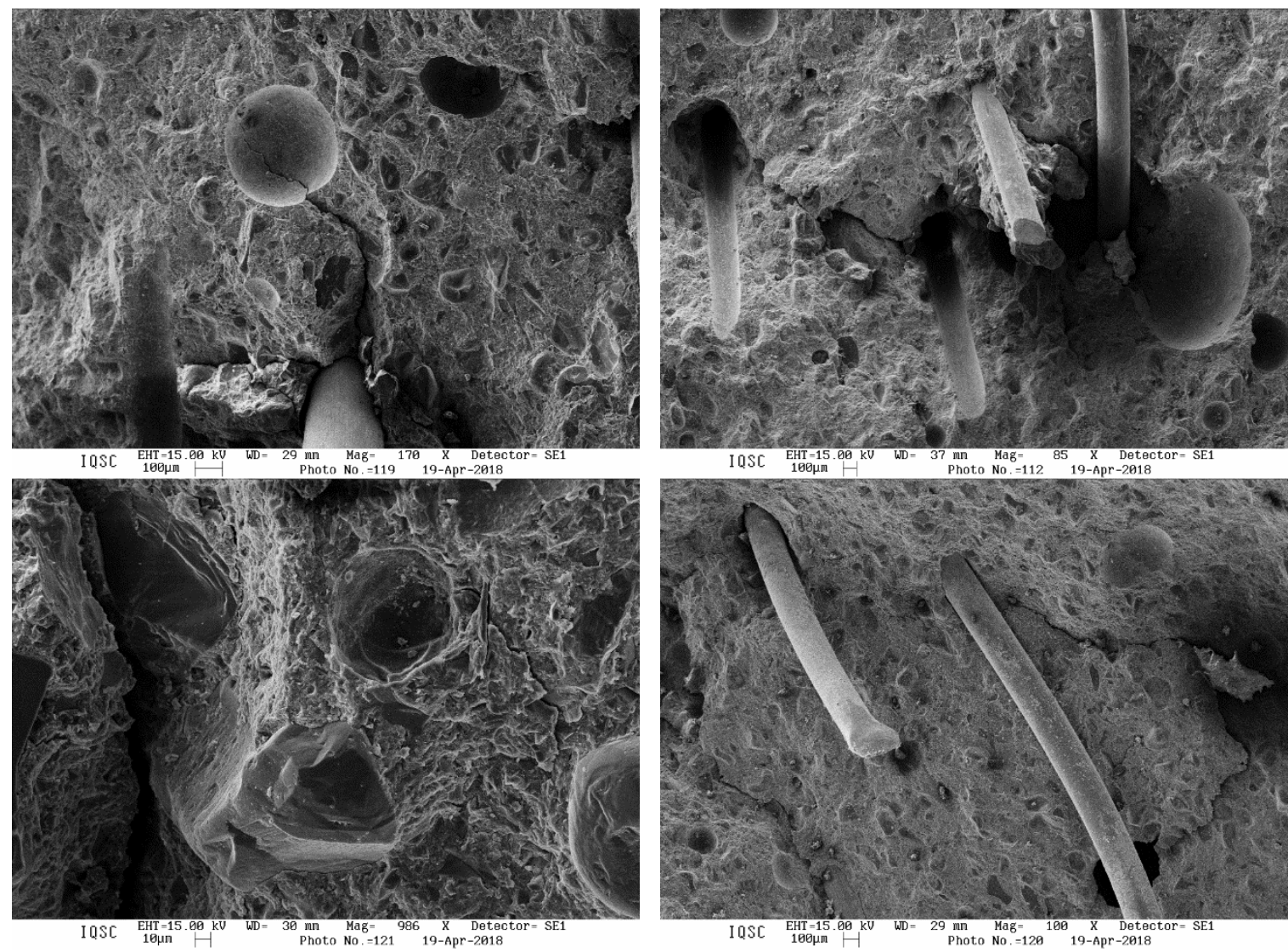

Figure 105 - Microcracking coalescence

These are some of the main damage mechanisms that occur and dissipate energy during severe loading. The quantification of such mechanisms can be made by cyclic tests which gives an estimative of the sum of the degradation process and do not can separate the phenomena. Acoustic emission test is prominent technic to separate the types of damage mechanisms occurred, (KRAVCHUK; LANDIS, 2018).

\subsection{Cyclic behavior and damage evolution in tension}

In Figure 106 it is possible to notice the progressive decay of the slope in unloading/reloading branches during cyclic tests performed with UHPFRC with $1 \%$ and $2 \%$ of fibers. Figure 107 shows the evolution of internal damage variable $d_{t}$, and its statistical analysis based on confidence and prediction bands. First cracking in tension occurred at a low level of strain in both ultra-high performance concrete (UHPC) and ultra-high performance fiber reinforced concrete (UHPFRC), around 0.00013. Some cycles were performed just after cracking. For UHPFRC with $1 \%$ of fibers, $d_{t}$ was equal to 0.18 at a strain of 0.00028 . For UHPFRC with $2 \%$ of fibers, $d_{t}$ was equal to 0.24 at a strain of 0.00019 . In this phase, the damage was higher for UHPFRC with more fibers. A possible explanation 
for this behavior is related to the interfacial transition zone (ITZ) between fibers and the paste. A higher amount of fibers result in a larger volume occupied by ITZ and consequently a higher degree of micro defects in the composite. The contribution of fibers to the strength is negligible in this phase. Therefore the evolution of damage in a matrix with more voids and defects occurs at higher rates. Another factor that may have some influence in this phase is the slightly lower volumetric ratio of the matrix when the fibers occupy a larger space. This first stage of behavior was maintained until a strain level of 0.00125 , when $d_{t}$ was equal to 0.6 and 0.7 , respectively in UHPFRC with $1 \%$ and $2 \%$ of fibers. This point was recognized as the beginning of multiple cracking formation and is marked in Figure 107.

During multiple cracking phase, the contribution of fibers was evident. UHPFRC with a higher volume of steel fibers contribute better to fiber bridging and consequently controlled better the damage evolution and localization of cracks in the matrix. At a strain of 0.01 , the damage variable was equal to 0.8 and 0.85 in UHPFRC with $1 \%$ and $2 \%$ of fibers. Consequently, the evolution of the damage variable during the multiple cracking phase was slower for the composite with $2 \%$ of discrete fibers, which can be explained by more efficient crack mitigation in the matrix.

Finally, the third phase of the behavior begins with the appearance of a visible macrocrack due to strain localization and a subsequent drop in load (softening branch). This process continued until the end of the test that occurred around a large strain (0.04). Fiber pullout was the predominant mechanism, as depicted in Figure 108 that shows the failure pattern for the gage length of the tensile specimen. In this phase, damage grew at lower rates for UHPFRC with $2 \%$ of fibers, probably because more fibers are crossing the main crack and then developing lower bonding stresses. At this branch, the composites carry similar loads for the two fiber contents. Therefore, the final damage variable was similar for both UHPFRC, $d_{t}=0.91$ with $1 \%$ of fibers and $\underline{d_{t}}=0.93$ with $2 \%$ of fibers. 

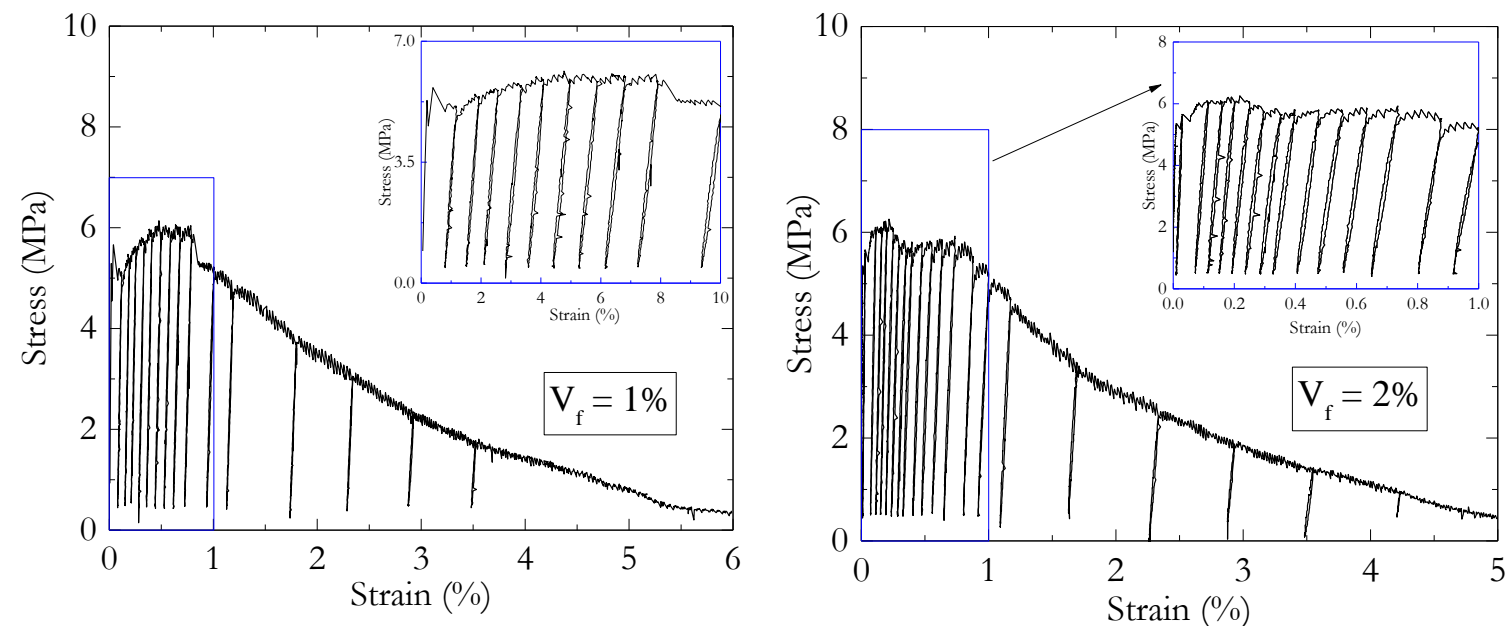

Figure 106 - Typical results of cyclic tension tests with $1 \%$ and $2 \%$ of fibers
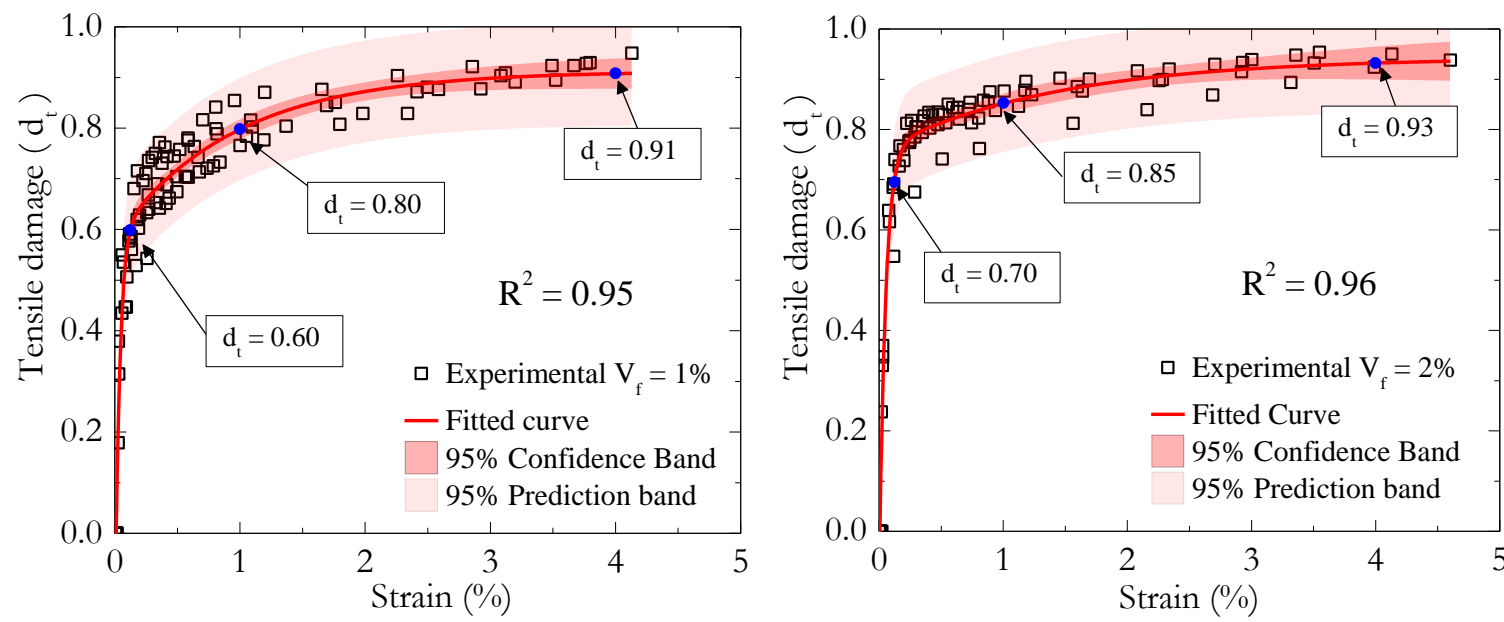

Figure 107 - Evolution of internal damage variable in tension for UHPFRC with $1 \%$ and $2 \%$ of fibers

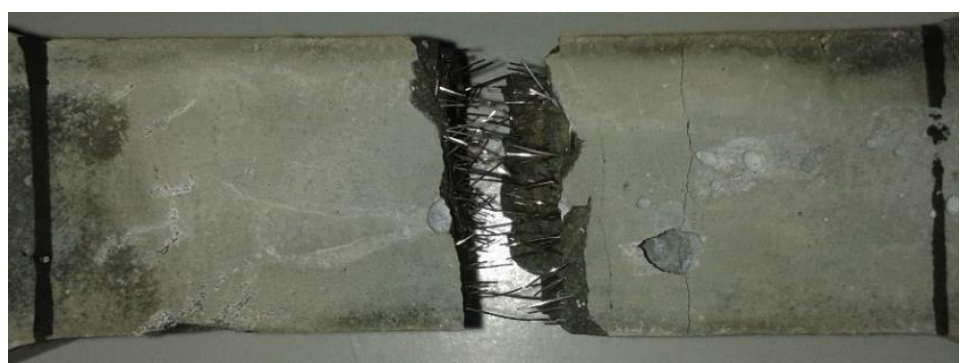

Figure 108 - Failure pattern of UHPFRC under tension

It can be seen from Figure 107 that the fitted curves can represent well the phenomena of damage evolution. The coefficient of determination was 0.95 and 0.96 for respectively UHPFRC with $1 \%$ and $2 \%$ of fibers. Besides, the figure also shows the confidence and prediction bands. Besides the upper bound prediction bands showed higher values than one, it is known that the damage is limited to one. 


\subsection{Cyclic behavior and damage evolution in compression}

Figure 109 shows the cyclic compressive stress-strain response for UHPFRC with $1 \%$ and $2 \%$ of fibers. The degradation evolution can be observed through the change in the inclination of the unloading-reloading branches of the curve. This evolution was quantified by the internal damage variable $d_{c}$, which is presented in Figure 110. Also, statistical analysis of the experimental results of $d_{c}$ is presented based on $95 \%$ confidence bands and $95 \%$ prediction bands. It can be seen in Figure 109 that UHPFRC presented an almost linear ascending branch of the stress-strain compressive behavior. Despite this, strain localization that occurs near the peak is preceded by early microcracking. Thus, besides less pronounced than in usual concretes, UHPFRC presents a nonlinear behavior before the peak load. The crack bridging and the stress transfer allows microcracking evolution until the peak load. At this moment strain localizes, and visible cracks appear, and consequently, a significant drop in load is registered. UHPFRC did not fail abruptly as opposed to UHPC. The measured damage index $d_{c}$ after this drop was 0.46 and 0.39 for UHPFRC with $1 \%$ and $2 \%$ of fibers, respectively. This stage is limited approximately by a strain value of 0.0075 . During the decrease in load, the fibers are more stressed in the macrocracks. Due to the lower number of fibers bridging these discontinuities in UHPFRC with $1 \%$ of fibers, higher bond and frictional stresses were probably developed in these fibers. This condition favors fiber debonding and slip that is a possible explanation for the higher damage index measured in UHPFRC with $1 \%$ fibers until this deformation stage. Also, the decrease in load capacity from peak until strain values of 0.0075 were $67.7 \%$ and $54.8 \%$ for respectively UHPFRC with $1 \%$ and $2 \%$ fiber content. Thus, the drop in load was more significant for the composite with less fibers that followed the same tendency of damage growth for this stage.

After the peak, the load capacity decreased at high rates due to the appearance of macrocracks. However, as the fibers were gradually activated by bridging cracks and the load supported became small, the rate of the damage evolution became smaller. The same was observed in the load dropping. The damage measured for UHPFRC with $1 \%$ of fibers was 0.61 at a strain of 0.015 (close the end of the test). For UHPFRC with $2 \%$ of fibers, $d_{c}$ was equal to 0.55 . These damage evolutions were reflected in the decrease in load. The decrease of load capacity for UHPFRC with $1 \%$ of fibers was $15.7 \%$. For UHPFRC with 2 $\%$ of fibers was $20.7 \%$. The higher rate of damage for UHPFRC with $2 \%$ of fibers can be attributed to the higher load carried by the composite during this stage. For this reason, 
degradation mechanisms between fiber and matrix are more prone due to the higher stresses developed in the fibers at macrocracks. Figure 111 presents the crack pattern for UHPFRC and UHPC at the end of the test.
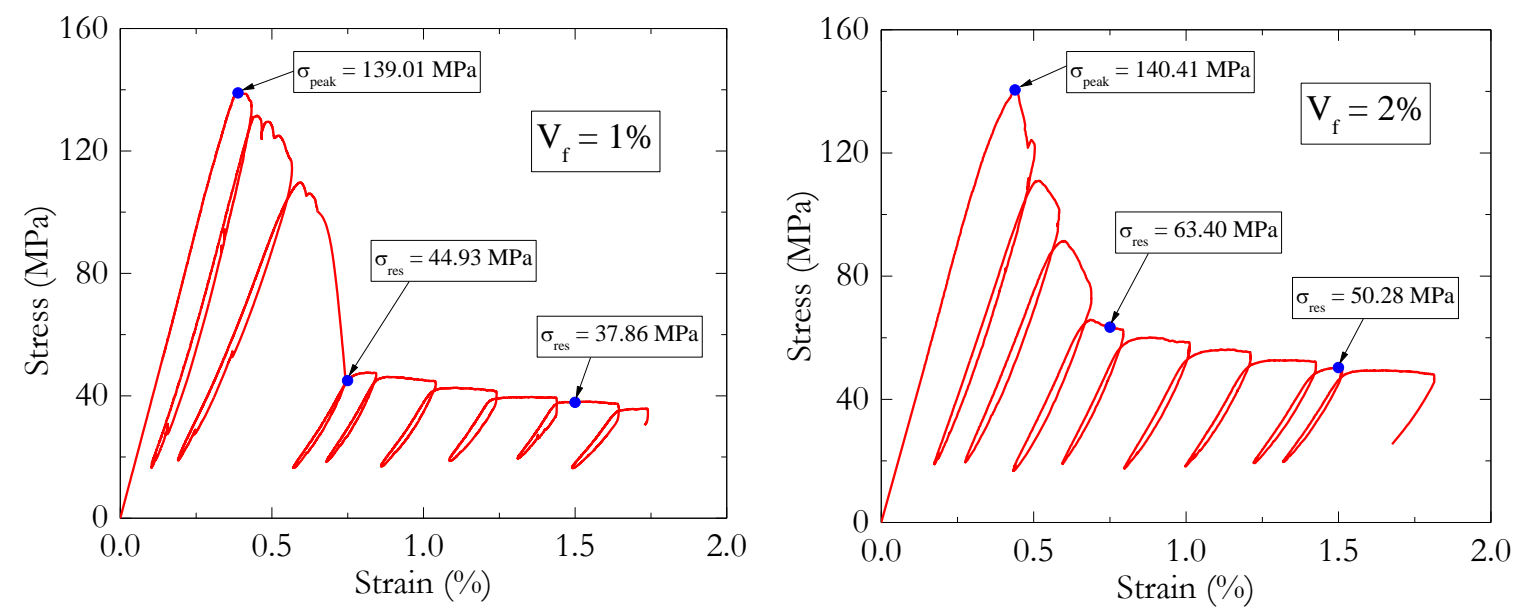

Figure 109 - Typical results of cyclic compression tests with $1 \%$ and $2 \%$ of fibers
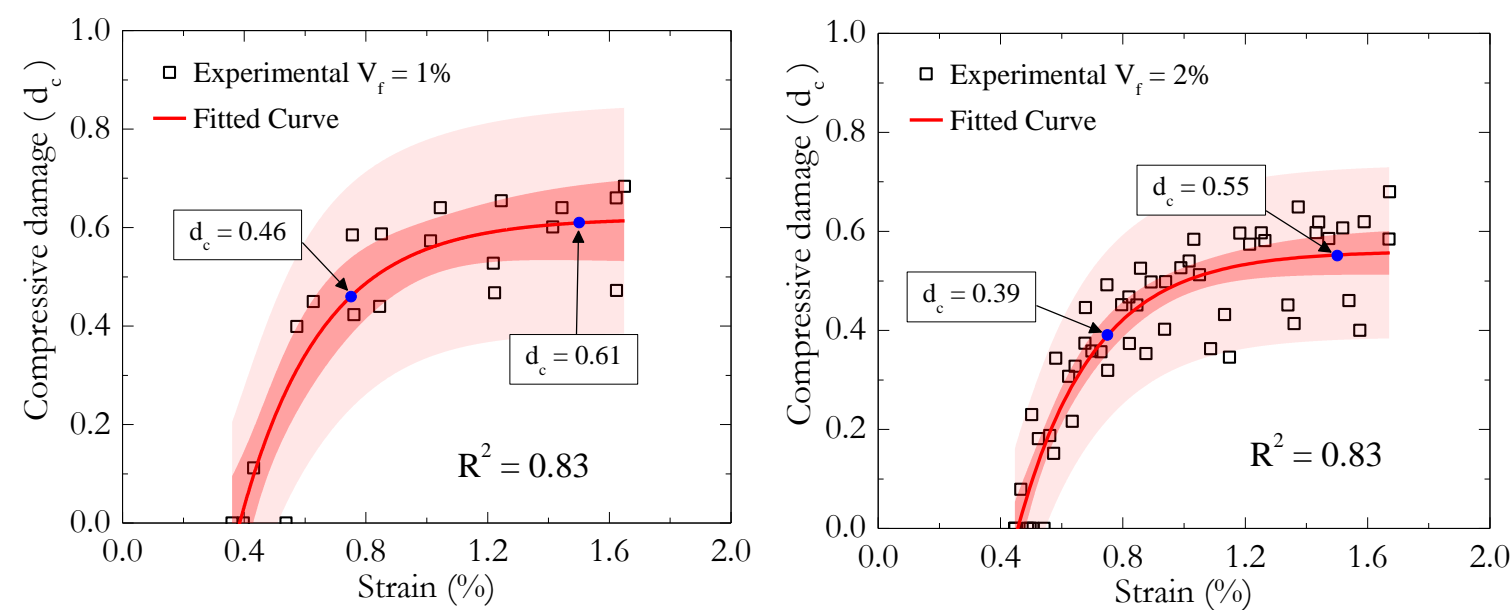

Figure 110 - Evolution of internal damage variable in compression with $1 \%$ and $2 \%$ of fibers

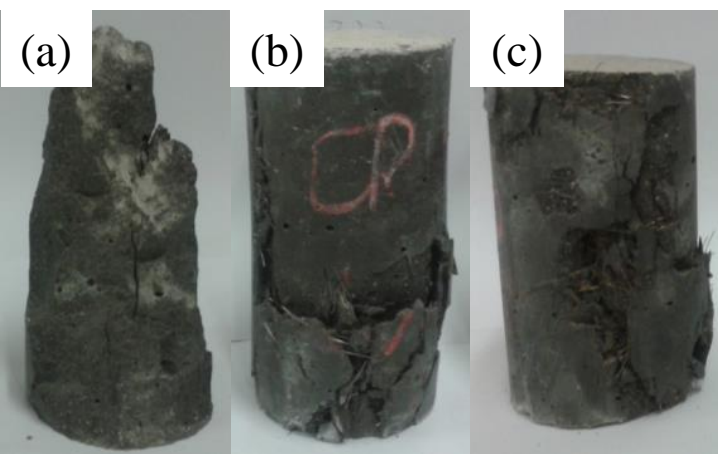

Figure 111 - Compressive failure patterns of (a) UHPC (b) UHPFRC with $1 \%$ of fibers and (c) UHPFRC $2 \%$ of fibers

Figure 110 shows that the adopted curve represents well the damage evolution of UHPFRC under compression. The coefficient of determination for the curves were 0.83 . 
However, the dispersion of the experimental data is higher than in tension, as can be seen in the prediction and confidence bands.

\subsection{Cyclic behavior under flexure}

The decay in stiffness of UHPFRC during cyclic flexure can be seen in Figure 112. Figure 113 presents the quantification of the degradation mechanisms through the global damage index $D_{g}$. Under displacement control, one cycle was performed after each $0.5 \mathrm{~mm}$. In the first cycle, the average global damage indexes registered were 0.42 and 0.47 for UHPFRC with $1 \%$ and $2 \%$ of fiber contents, respectively. This fast increase in damage variable is associated with the tensile nature of the formation of cracks which is unstable. Similar behavior was also observed in the direct tensile tests. Furthermore, the higher volume of fibers of UHPFRC with $2 \%$ fibers results in a greater volume of initial pores and microcracks, justifying the higher initial damage index measured.

At a displacement value of $1.5 \mathrm{~mm}$, the damage $D_{g}$ registered for UHPFRC with $1 \%$ and $2 \%$ of fibers were respectively 0.60 and 0.67 . The reduction of load capacity from the first registered cycle was $25.2 \%$ for UHPFRC with $1 \%$ fibers and $26.7 \%$ for UHPFRC with $2 \%$ fibers, which is consistent with the damage growth. The decay in bearing capacity measured between displacements of $1.5 \mathrm{~mm}$ and $3.5 \mathrm{~mm}$ was $72.2 \%$ and $58.4 \%$ for, respectively, UHPFRC with $1 \%$ and $2 \%$ fiber content. This tendency was also observed in damage growth, i.e., the damage variable increased more for UHPFRC with $1 \%$ of fibers, which was 0.84 and the value registered for UHPFRC with $2 \%$ fiber content was 0.84 . The greater increase in damage in this phase by UHPFRC with $1 \%$ of fibers can be attributed to the lower capacity of this composite of bridging cracks and delay the crack opening compared to UHPFRC with $2 \%$ of fibers. The lesser fiber content causes higher stresses in the fibers at cracks, and consequently, pullout phenomena prevail.

Also, it can be seen from Figure 112 that the area inside the hysteretic loops for UHPFRC with $2 \%$ is greater than for the composite with $1 \%$ of fibers. The occurrence of such hysteresis indicates energy dissipation during the unloading/reloading cycles. Thus, degradation mechanisms also occur during this stages. 

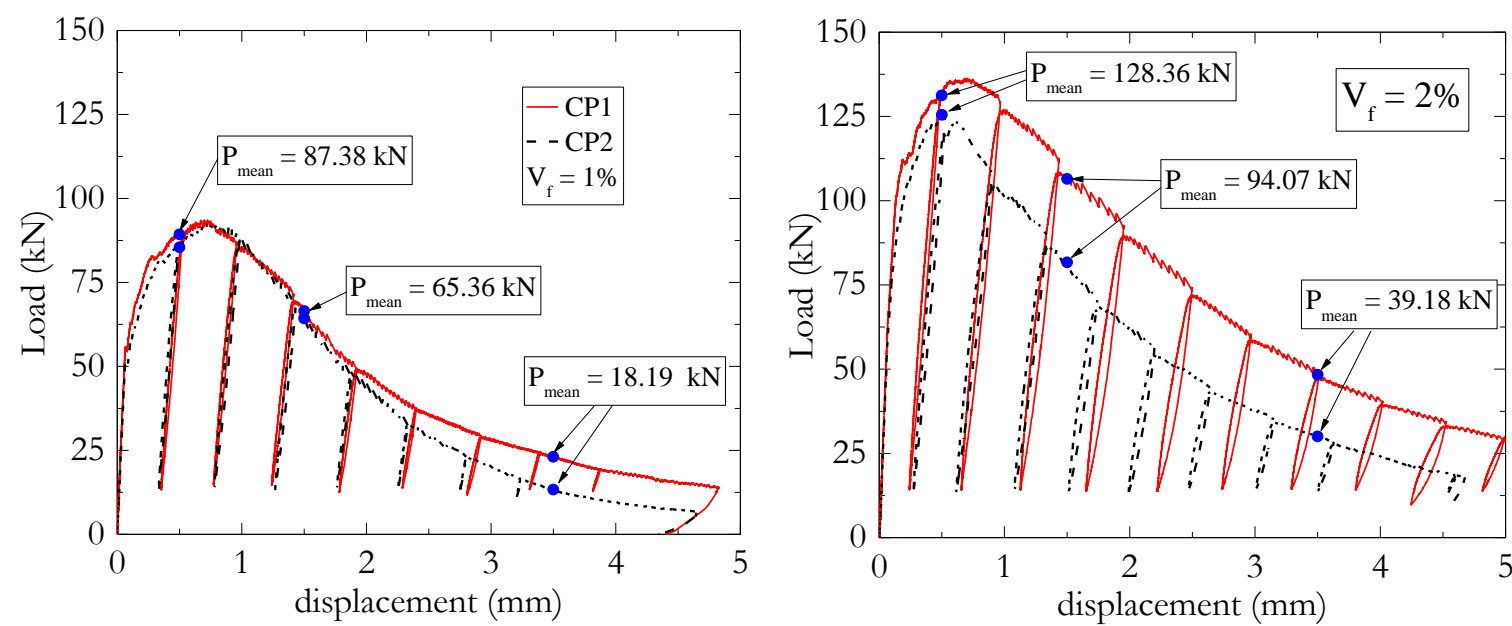

Figure 112 - Typical results of cyclic flexure tests with $1 \%$ and $2 \%$ of fibers
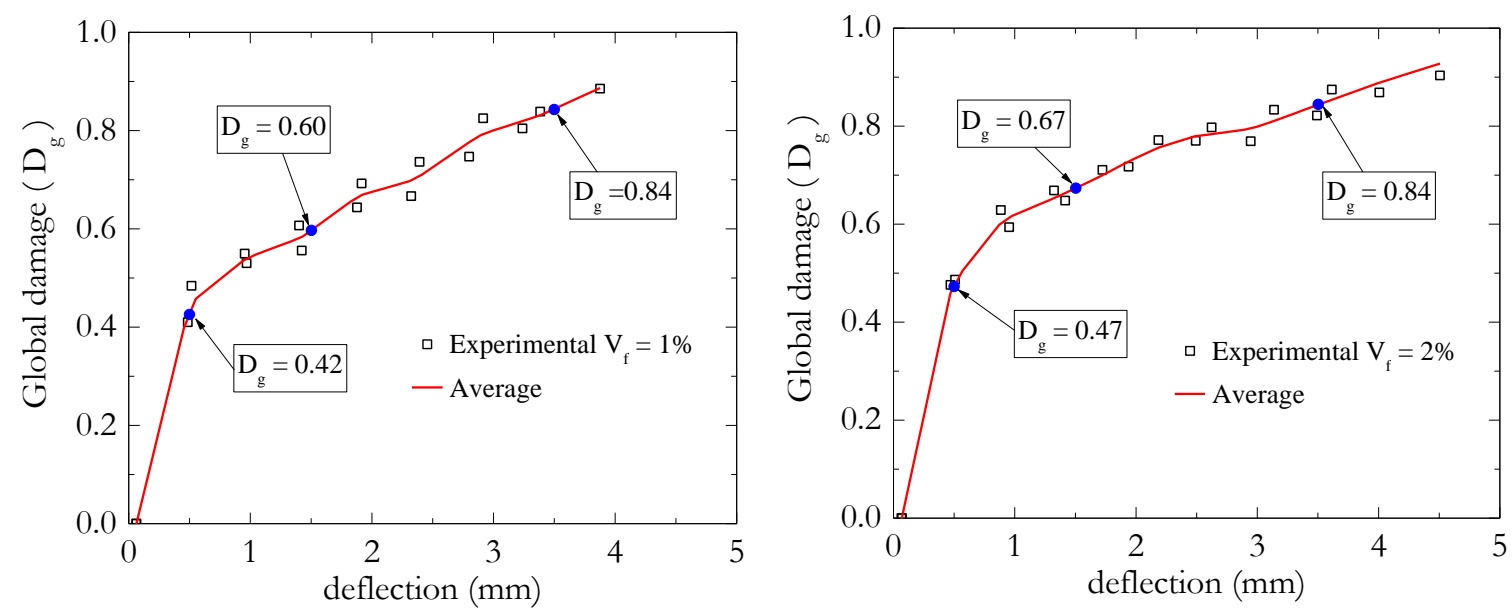

Figure 113 - Experimental global damage evolution for the tested prisms

\subsection{Lateral stability of UHPFRC beams lifted by cables}

Two beams of UHPFRC were tested under lifting conditions with inclined cables. At the moment of the test, the beams had 28 days. The material corresponds to Batch 2 described in item 4.1. The flexural behavior was presented in topic 5.3 and the compressive behavior in topic 5.2. In item 4.9, the instrumentation was described. Remembering, six LVDTs were fixed to measure longitudinal strain at midspan of the UHPFRC beams. This information enables to determine the critical sectional behavior and also determine the exact moment of cracking. The modulus of elasticity of UHPFRC was $40.2 \mathrm{GPa}$, and the tensile stress capacity obtained from the four-point bending test was $11.2 \mathrm{MPa}$. Thus, the average cracking strain obtained from this results is $2.79 \times 10^{-4}$.

The results are presented for each beam regarding load versus longitudinal strain and load versus midspan rotation. The strains are from LVDTs measurements and the rotation from the interpretation of the total station results. Figure 114 (a) presents the load versus 
longitudinal strains for the LVDTs 1 to 3 that were positioned on one side of the beam 1 , and Figure 114 (b) that gives the results of the LVDTs 4 to 6 in the other side of the beam. The buckling load for such element was $9.81 \mathrm{kN}$ with a tensile strain of $5.17 \times 10^{-4}$ at this time, registered by the LVDT 3 located near the bottom face of the beam.

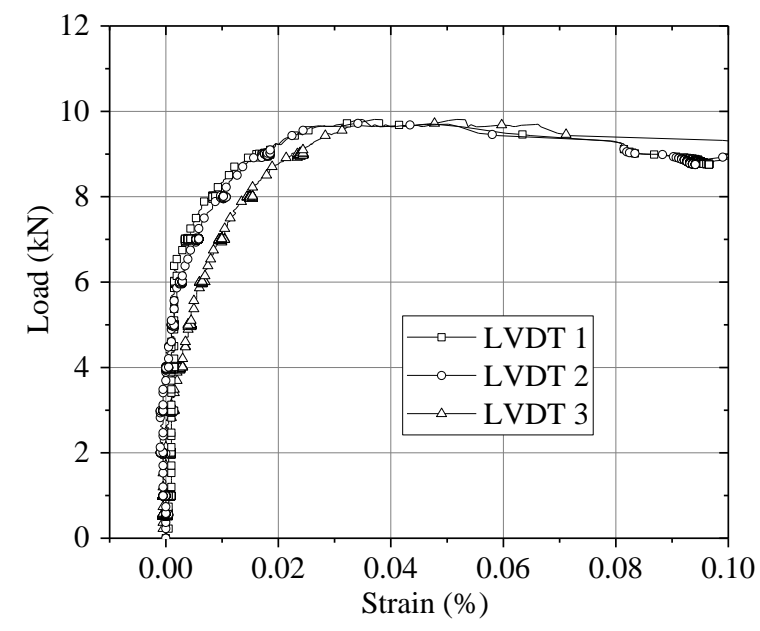

(a)

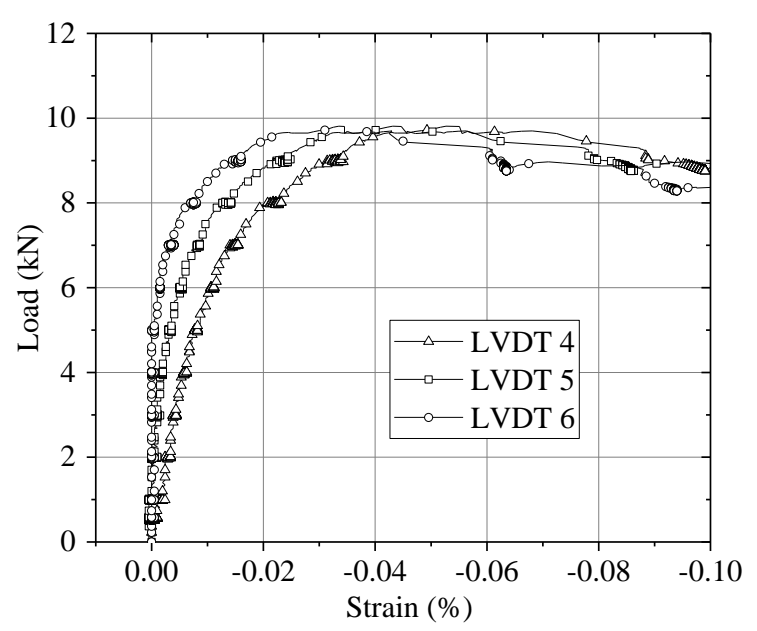

(b)

Figure 114 - (a) Tensile and (b) compressive strains developed in the middle span section of beam

1

As already presented, the cracking strain obtained from four-point bending tests was $2.78 \times 10^{-4}$ that is taken as a reference to detect cracking of the lifted beams. Then, the cracking load occurred close to the peak load for beam 1. Thus, the nonlinear regime registered in the pre-peak branch is attributed mainly to large displacements with the material behaving elastically. At the peak, the maximum compressive strain was $-5.26 \times 10^{-4}$, registered by the LVDT 4 near the top flange. This value is small compared to the compressive first cracking strain. Furthermore, from the figures, it is clear that at early stages of load, around $4 \mathrm{kN}$, tensile strains prevailed at one side of the beam (LVDTs 1, 2 and 3) and compressive strains prevailed (LVDTs 4, 5 and 6). This behavior indicates the gradual appearance of a lateral component of the load acting in the minor axis of inertia and causing lateral flexure. The developed tensile strains at peak load were insufficient to yield the steel bars. Figure 115 (a) and (b) present the load versus tensile and compressive strains for beam 2 , respectively. 


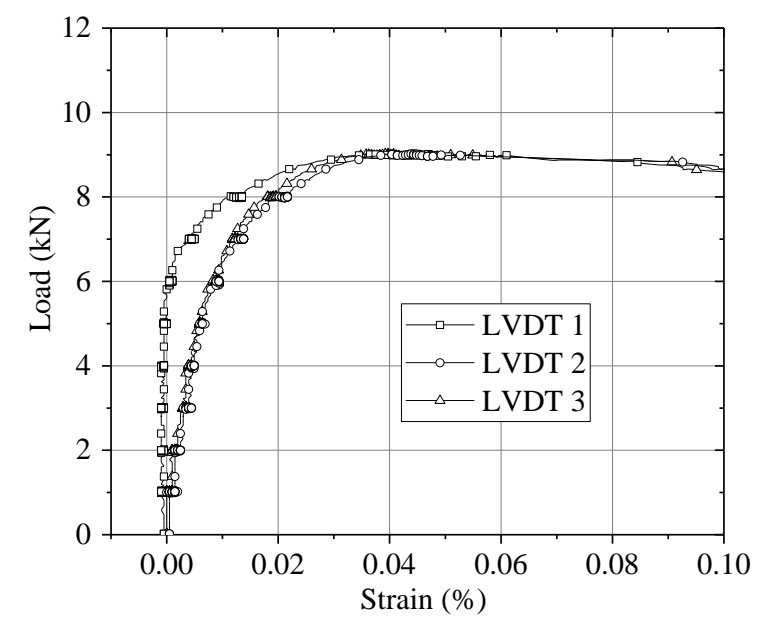

(a)

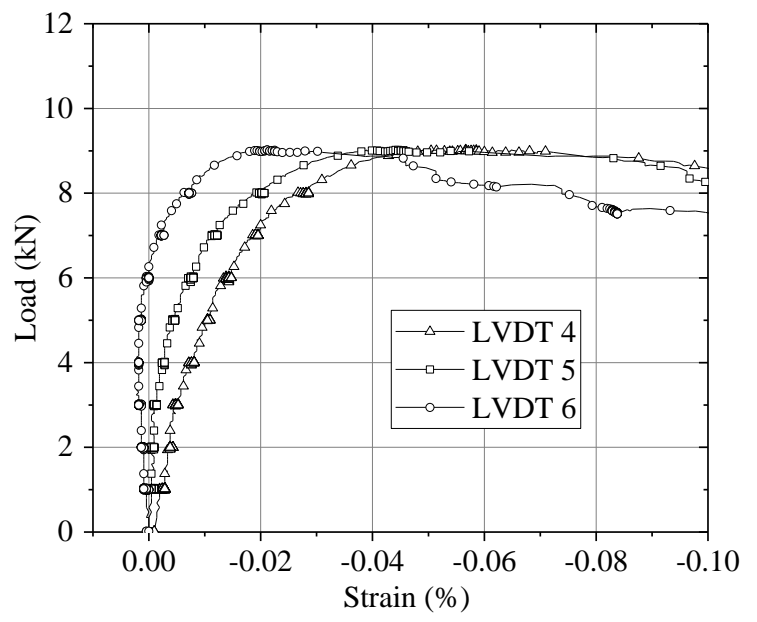

(b)

Figure 115 - (a) Tensile and (b) compressive strains developed in the middle span section of beam 2

The buckling load for this beam was $9.03 \mathrm{kN}$ that differenced by $7.95 \%$ from the beam 1 was. The maximum tensile strain at peak was $4.23 \times 10^{-4}$ that is greater than the cracking strain. Thus, the beam cracks before the peak load. However, it can be seen from the Figure 115 (a) that the beam cracks near the peak load. Thus, large displacements prevailed in the pre-peak stage. The beam 2 also started to rotate early due to its high slenderness, then at one side prevails compressive strains and at the other side tensile strains. The combination of major and minor axis flexural strains resulted in higher tensile strains on the bottom face of the beams at LVDT 3. It can be seen from Figure 114, and Figure 115 that all the measured strains passed to increase at higher rates after the load of $6 \mathrm{kN}$ and the load passed to increase at smaller rates, indicating an instability behavior. Comparatively, if these same beams were braced over the span, the concentrated load required to mobilize its flexural strength would be approximately $35 \mathrm{kN}$. This flexural capacity was calculated according to the model presented in (FEHLING et al., 2014). Also, the preponderant lateral flexure observed in the plot of strains was also reflected in the cracking pattern that was distributed in the central part of the beam in the vertical direction at the most tensioned side, Figure 116. 


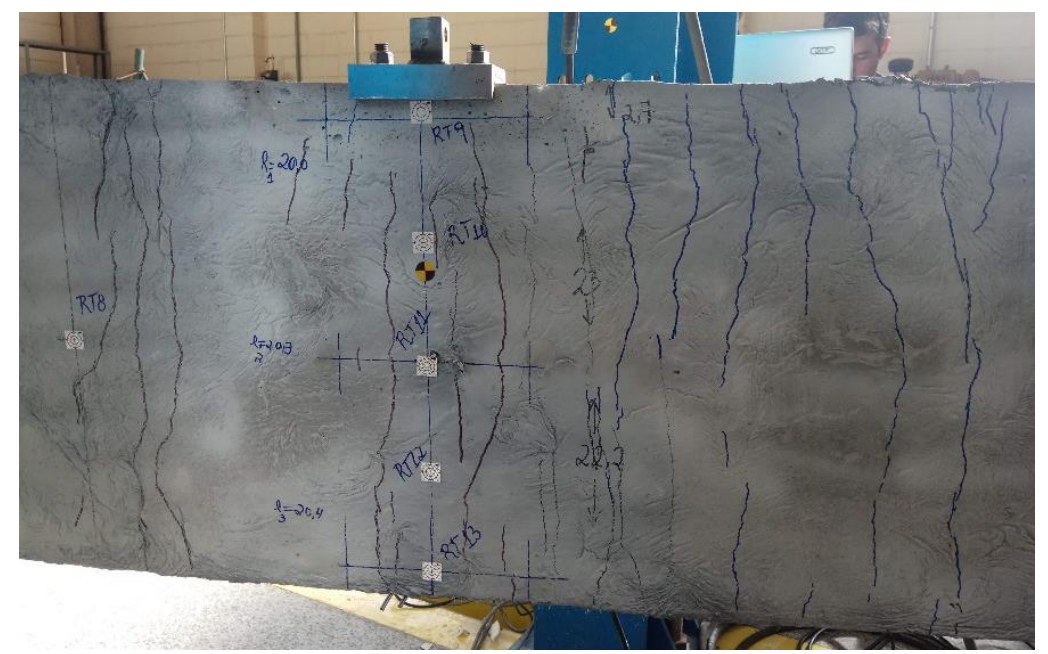

Figure 116 - Cracking pattern

The width variation, the eccentricity of the lifting points, the variability of concrete strength and initial imperfections can be attributed to the observed difference in the peak load between the two beams. The last factor has a significant effect on the lateral stability of beams, as is well known. Thus, to investigate this variable, the total station equipment enabled to measure the initial configuration of the beams. Figure 117 presents the initial sweep of the beams 1 and 2, respectively. The centroid of the beam at the two lifting positions was considered as the origin of ordinate axis.

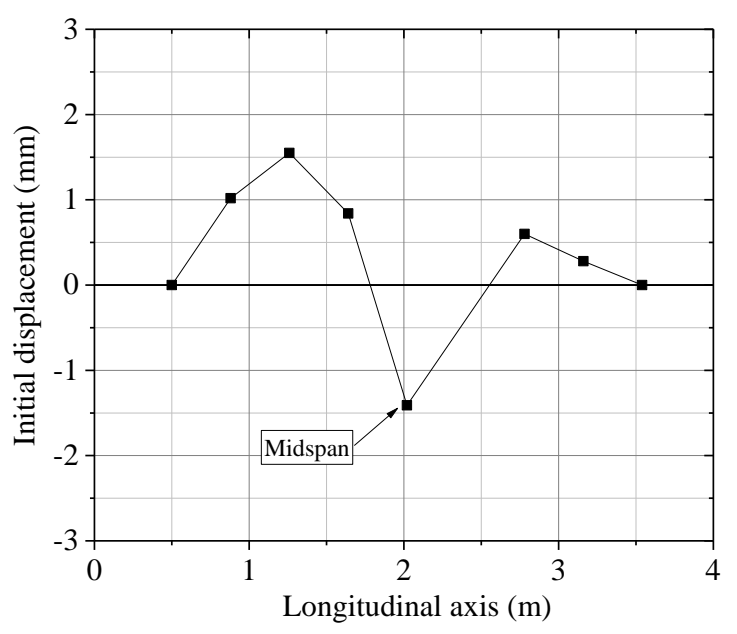

(a)

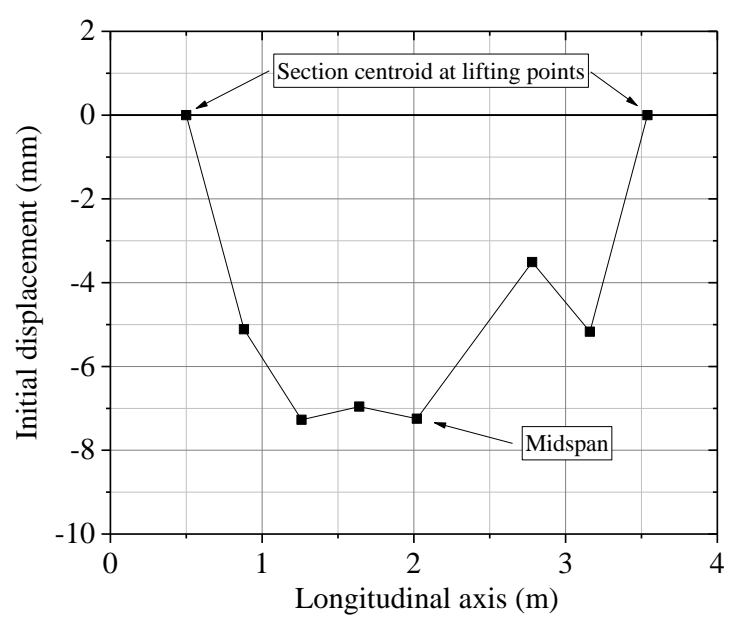

(b)

Figure 117 - Initial imperfections of (a) beam 1 and (b) beam 2

Thus, one possible reason for beam 1 has presented the higher peak load is its smaller initial imperfection compared to beam 2 . Also, the first beam presented sweep for both sides of the axis with similar magnitudes. This shape compared to the beam 2 seems less favorable to the increase in lateral displacements and rotation. Both beams toppled in the negative direction. 
The rotation evolution of the beams during the test was determined from the vectors recorded by the total station at each stop of the load.

$$
\sin \varphi=\frac{z_{1}-z_{2}}{\sqrt{\left(x_{1}-x_{2}\right)^{2}+\left(y_{1}-y_{2}\right)^{2}+\left(z_{1}-z_{2}\right)^{2}}}
$$

Where $\varphi$ is the angle of rotation, $\left(x_{1}, y_{1}, z_{1}\right)$ and $\left(x_{2}, y_{2}, z_{2}\right)$ represents the coordinates of the point of reflective tapes positioned near the top and bottom face of the section of interest, respectively. Figure 118 presents the load-rotation curves for beams 1 and 2, respectively. The rotations were calculated for the lifting points and at midspan. These plots enable to verify possible torsional strains developed by the relative twist and initial rotations.
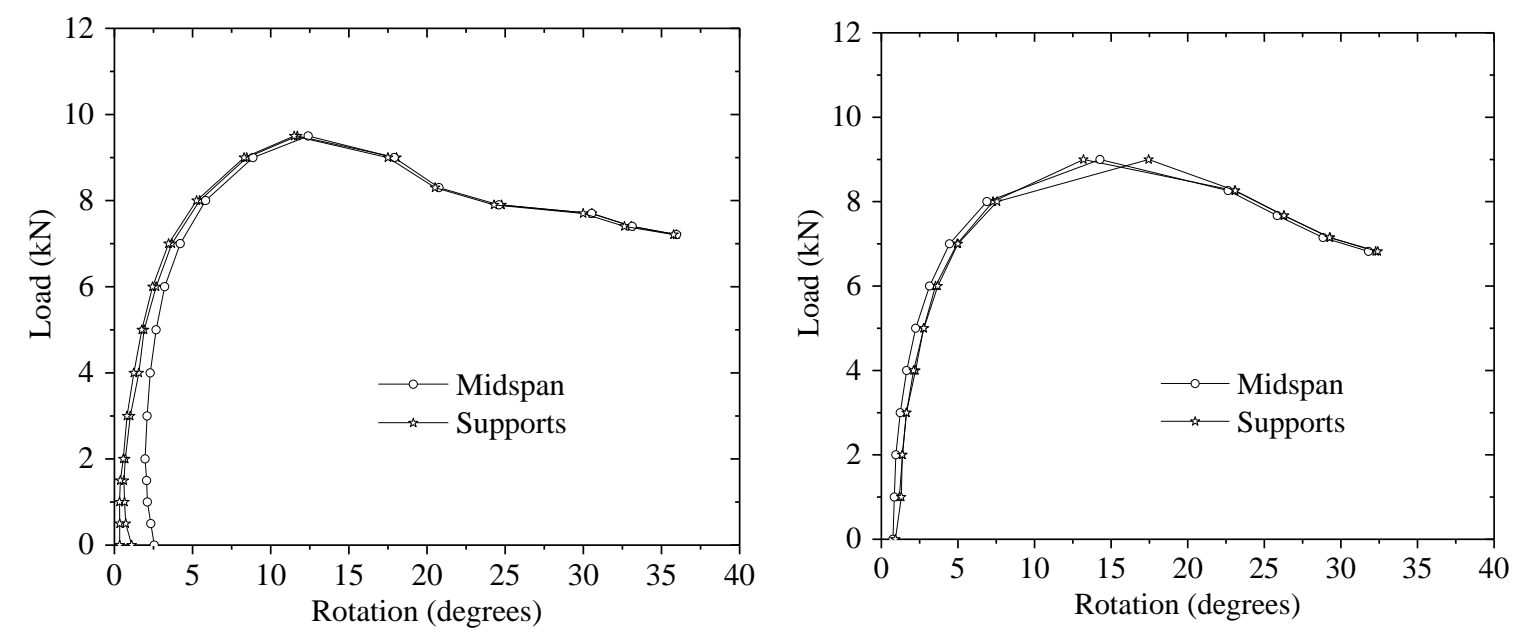

Figure 118 - Load-rotation curves for beams 1 and 2, respectively

Beam 1 presented an initial rotation of 2.55 degrees and beam 2 presented 0.76 degrees. However, the increase in the rotation was faster for beam 2 that can be attributed to the greater initial lateral displacement of the element and its initial shape, which seems favorable for increase the twist. The beam 1 rotated initially against the side that toppled at the end of the test. This behavior indicates the influence of the initial shape of the element. Furthermore, torsional twist due to relative rotation between supports and midspan can be considered as small, even after cracking. Thus, it can be concluded that the beams presented lateral flexural behavior combined with preponderantly rigid body rotation. At the peak load, the rotation of the beam 1 was 12.5 degrees, and the beam 2 was 14.2 degrees. Figure 119 presents the stages of rotation and the correspondent load for the beam 2 in the pre-peak range. 


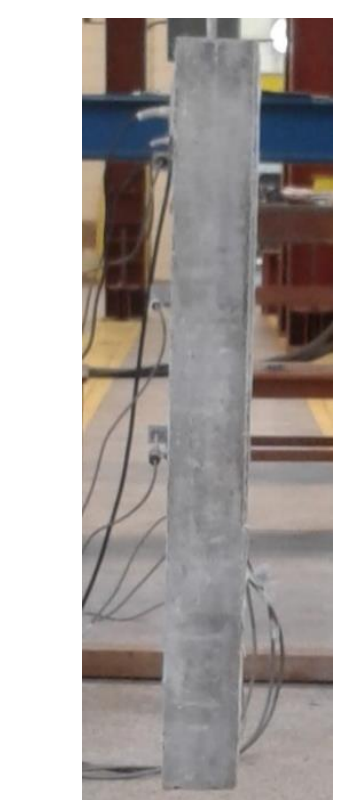

$\varphi=0.76^{\circ}$

$\mathrm{Q}=0 \mathrm{kN}$

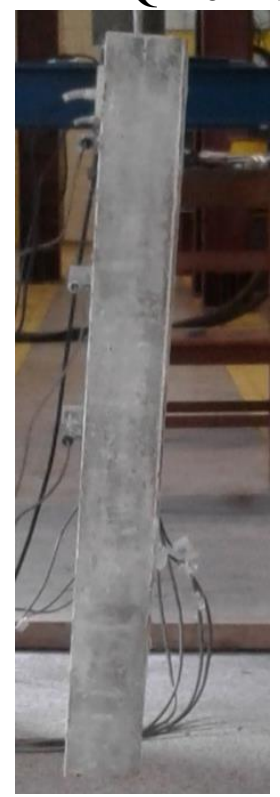

$\varphi=3.15^{\circ}$

$\mathrm{Q}=6 \mathrm{kN}$

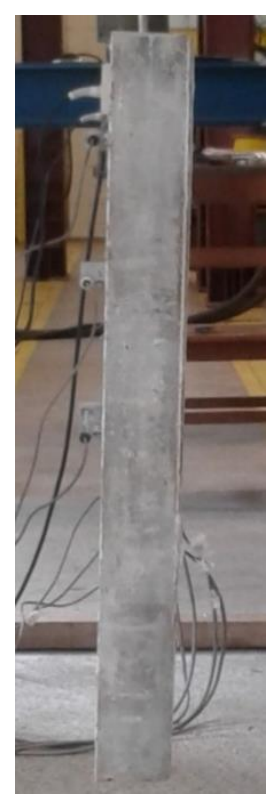

$$
\begin{aligned}
& \varphi=1.65^{\circ} \\
& Q=4 \mathrm{kN}
\end{aligned}
$$

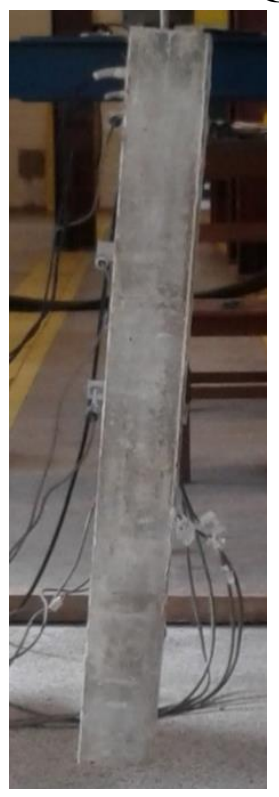

$\varphi=4.46^{\circ}$

$\mathrm{Q}=7 \mathrm{kN}$

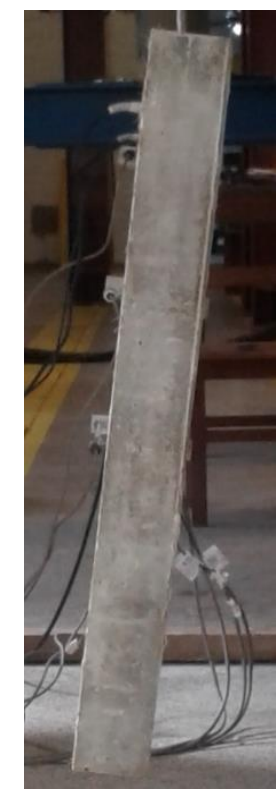

$\varphi=6.89^{\circ}$

$\mathrm{Q}=8 \mathrm{kN}$

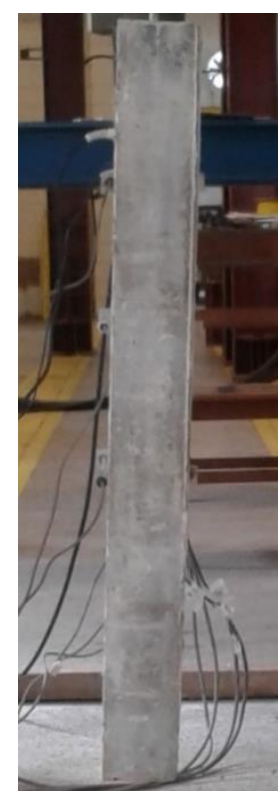

$$
\varphi=2.24^{\circ}
$$$$
\mathrm{Q}=5 \mathrm{kN}
$$

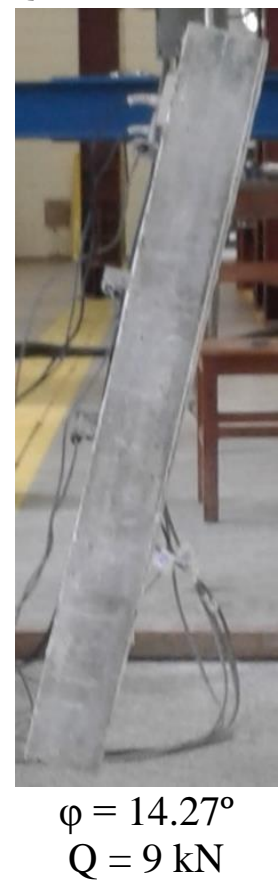

Figure 119 - Stages of rotation of beam 2

The UHPFRC beam started to experience large rotations with the increase in load permitted by the flexible supports. However, the load capacity was significantly limited by the boundary conditions. The decrease in load after the peak is associated with the beginning of cracking, but such decrease was gradual due to the presence of fibers in the concrete mix. Figure 120 presents global views of the beam at the end of the test. 

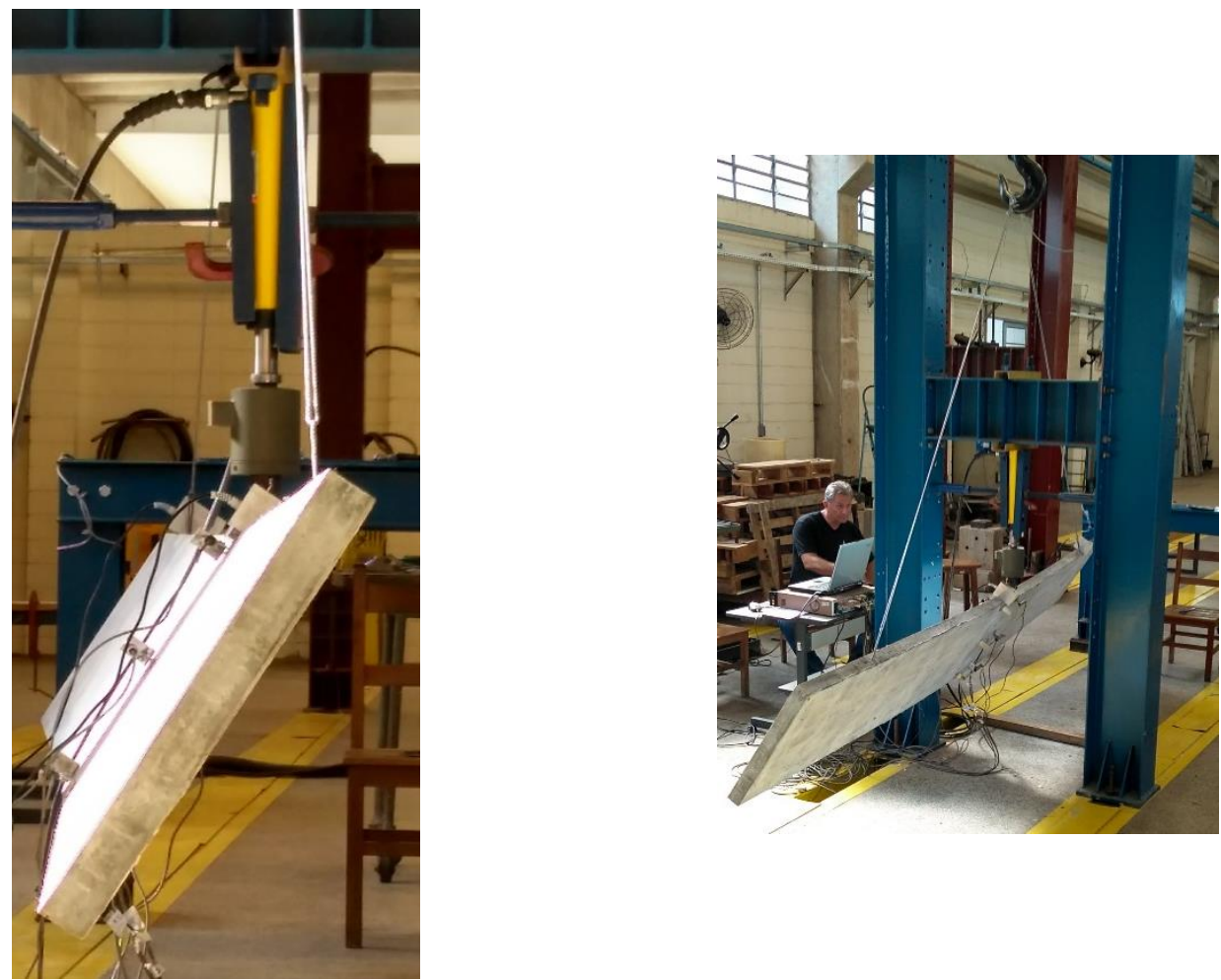

Figure 120 - Beam rotation at the end of the test

Besides the visible rigid body rotation, the lateral curvature of the UHPFRC element also can be seen. Figure 120 presents this same final state for the results provided by the total station measurements. Again, the position of the total station was at the coordinate $\mathrm{X}_{\mathrm{P}}$ $=5000.0 \mathrm{~m}, \mathrm{Y}_{\mathrm{P}}=1000.0 \mathrm{~m}$, and $\mathrm{H}_{\mathrm{P}}=100.0 \mathrm{~m}$.

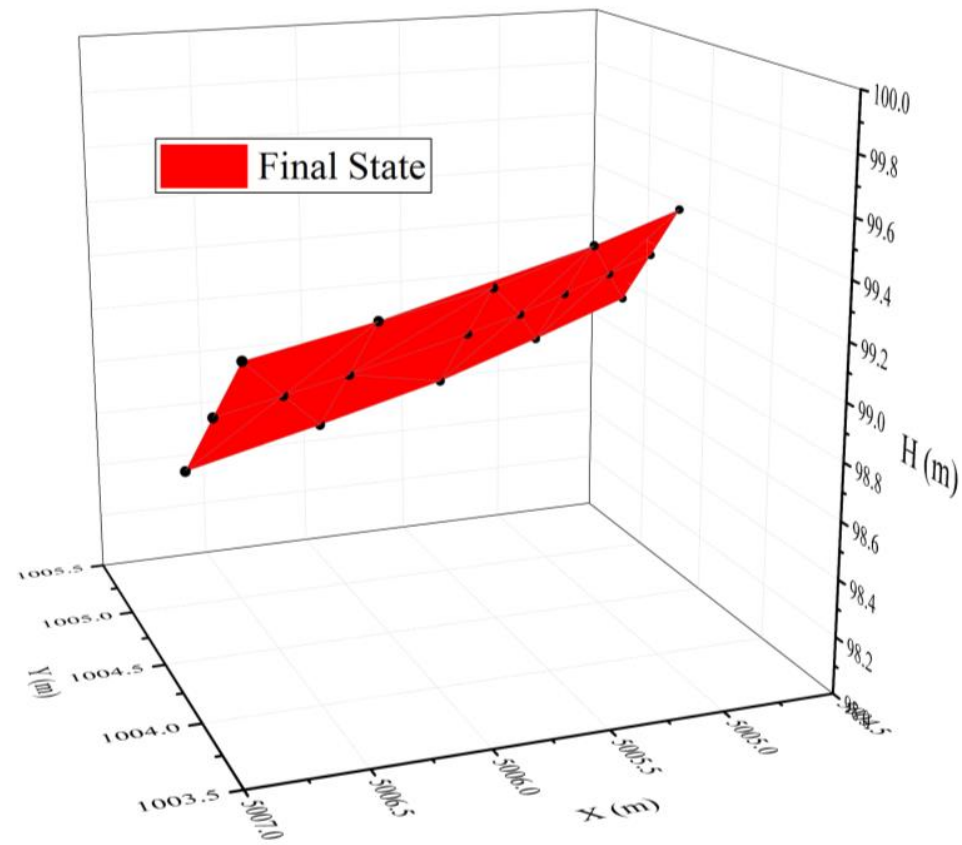

Figure 121 - Final configuration of the UHPFRC beam 


\section{ANALYTICAL PROPOSALS}

\subsection{FOR UHPFRC}

\subsubsection{Compressive stress-strain behavior}

The model of (CARREIRA; CHU, 1985) is utilized to represent the entire stressstrain behavior of UHPFRC under compressive load. Is already known that the model is capable of predicting very well the pre-peak behavior of fiber reinforced concrete with different grades. Furthermore, (MANSUR; CHIN; WEE, 1999) further developed the solution for the post-peak range by adding two constants in the equation to simulate the influence of the addition of fibers, equation (99). The constants are only considered in the post-peak branch.

$$
\begin{gathered}
\sigma=\sigma_{0}\left[\frac{k_{1} \beta \frac{\varepsilon}{\varepsilon_{0}}}{k_{1} \beta-1+\left(\frac{\varepsilon}{\varepsilon_{0}}\right)^{k_{2} \beta}}\right] \\
\beta=\frac{1}{1-\frac{\sigma_{0}}{\varepsilon_{0} E_{c 0}}}
\end{gathered}
$$

Where $\sigma_{0}, \varepsilon_{0}, E_{c 0}$ are the peak stress, peak strain and initial modulus of elasticity, respectively, which are known parameters. $k_{1}$ and $k_{2}$ are experimental-based parameters determined concerning the post-peak range of experimental results. (MANSUR; CHIN; WEE, 1999) proposed equations for these variables dependent on the volume aspect ratio of the fibers and the concrete peak stress $\sigma_{0}$. In the present study, $k_{1}$ and $k_{2}$ were calibrated for the parameters $\beta$, as in equation (55), and the total energy $W_{c}$ that corresponds to the mean area of the experimental stress-strain diagrams, see equations (56) and (57).

$$
\begin{aligned}
& k_{1}=0.42539-0.04942 \beta-0.2071 W_{c}+0.00186 \beta^{2}+0.16163 W_{c}^{2} \\
& k_{2}=1.19603-0.09059 \beta-0.82313 W_{c}+0.00329 \beta^{2}+0.37862 W_{c}^{2}
\end{aligned}
$$

Figure 122 presents the comparison between experimental results for UHPFRC with $1 \%$ and $2 \%$ fibers and the analytical solutions from equations (55), (56) and (57). 

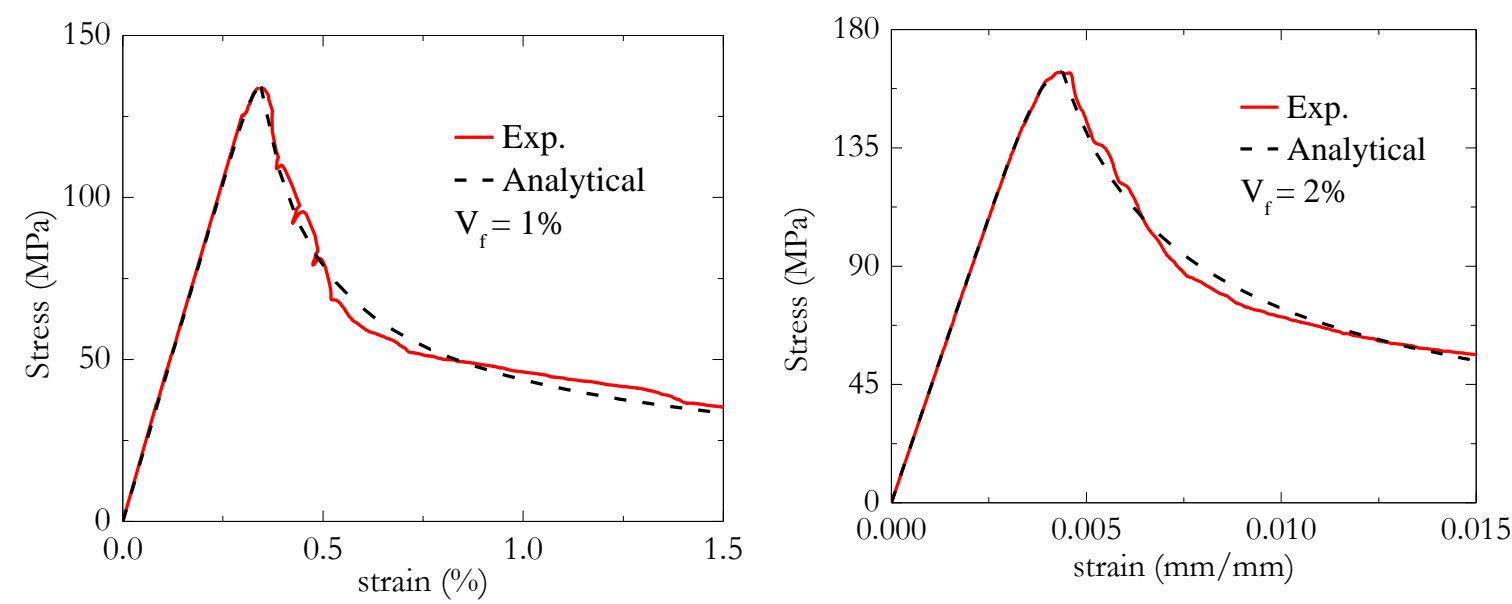

Figure 122 - Comparison of analytical solutions and experimental results for compression tests

As mentioned above, equation (55) without the parameters $k_{1}$ and $k_{2}$ describes well the pre-peak branch without the need for any calibration. Besides, the post-peak range was accurately represented by equation (55) in ductility and toughness for different fiber contents.

\subsubsection{Tensile stress-strain behavior}

As mentioned earlier, distinct mechanisms occur during multiple cracking phase and localization stage. However, as a simplifying hypothesis, a homogeneous and isotropic behavior was assumed for UHPFRC until failure in numerical simulations carried out. These assumptions are convenient for most constitutive models that do not represent cracks discretely. Thus, dividing the displacement measured experimentally until rupture by the gauge length enables to obtain an equivalent homogeneous strain. The total energy $W_{t}$ consumed in these two inelastic stages of behavior corresponds to the area under the experimental curve. Here, $W_{t}$ is the average area of all experimental diagrams for each fiber content. Therefore, equation (58) was chosen to represent the stress-strain behavior of UHPFRC under tension which is based on the concept of total energy. This equation is a Gaussian function which starts at the cracking strain.

$$
\sigma_{t}(\varepsilon)=\frac{W_{t}}{I_{p} \sqrt{\pi / 2}} e^{\frac{-2\left(\varepsilon-\varepsilon_{p}\right)^{2}}{I_{p}{ }^{2}}}+\sigma_{u}
$$

The variables presented in equation (58) are shown in Figure 123, in which $I_{p}$ represents the inflection point of the curve at the descending branch, $\varepsilon_{p}$ the peak strain, and 
$\sigma_{u}$ the stress value measured at the end of the test. Besides, the cracking stress and strain are shown in Figure 123, which are known in advance.

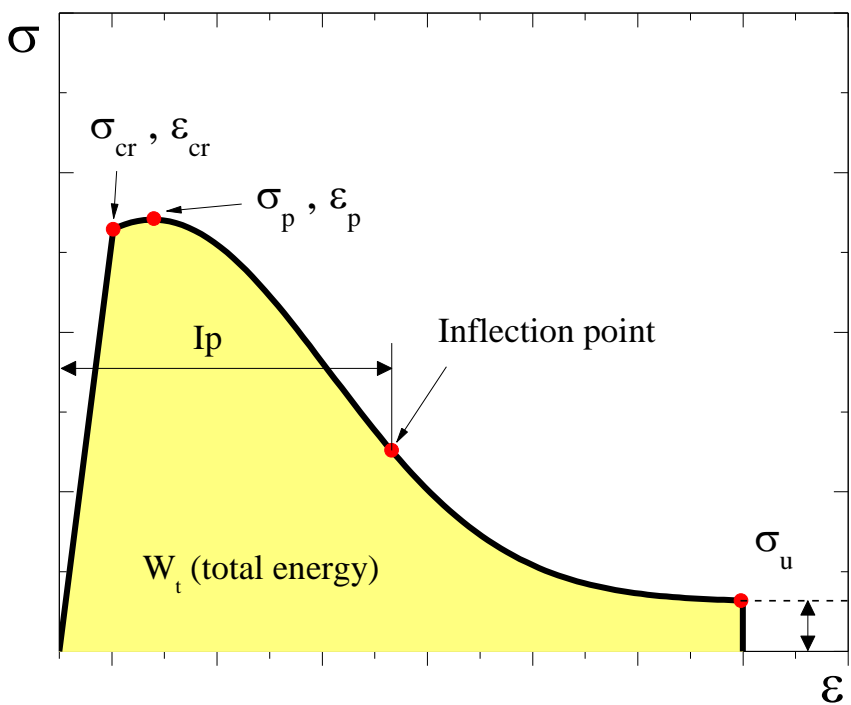

Figure 123 - Representation of the analytical model variables

The model adopted and shown in Figure 123 can represent the strain hardening behavior and the softening branch of the uniaxial tensile response of UHPFRC. Figure 124 (a) and (b) present the comparison between the analytical solution and experimental envelopes for UHPFRC with $1 \%$ and $2 \%$ of fibers.

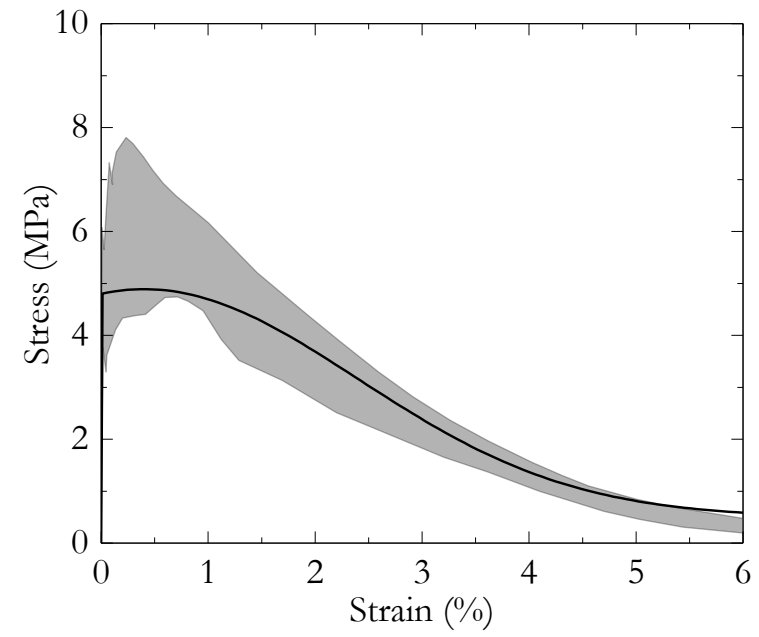

(a)

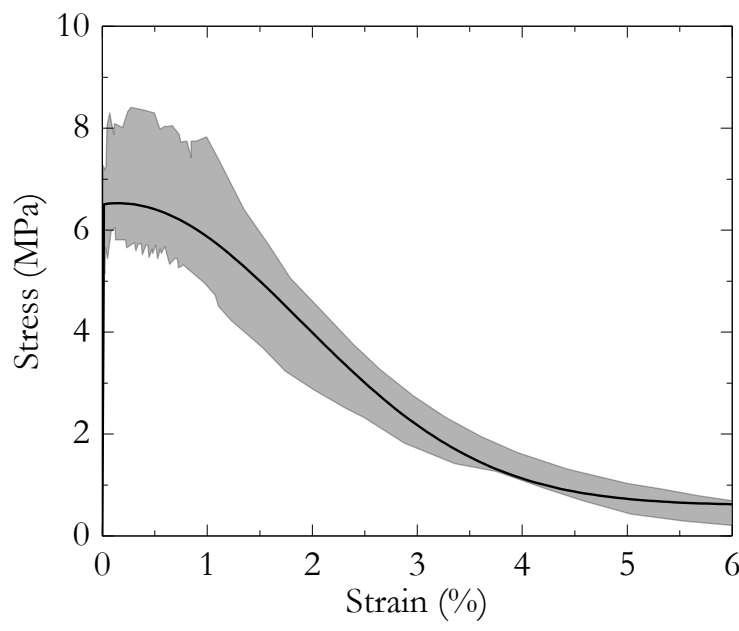

(b)

Figure 124 - Comparison between tensile experimental results and analytical solution for (a) UHPFRC with $1 \%$ of fibers and (b) UHPFRC with $2 \%$ of fibers

The energy-based analytical solution represents the ductility and toughness (the area below the curve) of UHPFRC accurately under tension load and for different fiber contents. 


\subsubsection{Damage evolution in compression}

The fitting curves presented in Figure 110 are exponential functions of the form:

$$
d_{c}(\varepsilon)=y_{0}+A_{1} e^{-\varepsilon / t_{1}}
$$

Table 14 presents the constants of equation (59) for prediction of the compressive damage evolution.

Table 14 - Parameters for compressive damage evolution, equation (60)

\begin{tabular}{ccc}
\hline Damage & \multicolumn{2}{c}{ Compressive } \\
\hline Fiber content & $1 \%$ & $2 \%$ \\
\hline $\mathrm{y}_{0}$ & 0.61931 & 0.56049 \\
$\mathrm{~A}_{1}$ & -2.58946 & -3.57839 \\
$\mathrm{t}_{1}$ & 0.00269 & 0.00246 \\
\hline
\end{tabular}

The proposed equation is a simple fit of the experimental data. Thus, an equation with constants with physical meaning is more suitable and are recommended for future work.

\subsubsection{Damage evolution in tension}

The fitting curves presented in Figure 107 are exponential functions of the form:

$$
d_{t}(\varepsilon)=y_{0}+A_{1}\left(1-e^{-\varepsilon / t_{1}}\right)+A_{2}\left(1-e^{-\varepsilon / t_{2}}\right)
$$

Table 15 presents the constants $y_{0}, A_{1}, A_{2}, t_{1}$ and $t_{2}$ of equation (60) for the prediction of tensile damage evolution.

Table 15 - Parameters for tensile damage evolution, equation (60)

\begin{tabular}{ccc}
\hline Damage & \multicolumn{2}{c}{ Tensile } \\
\hline Fiber content & $1 \%$ & $2 \%$ \\
\hline $\mathrm{y}_{0}$ & -0.22146 & -0.11437 \\
$\mathrm{~A}_{1}$ & 0.80523 & 0.87236 \\
$\mathrm{~A}_{2}$ & 0.3286 & 0.18508 \\
$\mathrm{t}_{1}$ & $3.66896 \mathrm{E}-4$ & $5.0235 \mathrm{E}-4$ \\
$\mathrm{t}_{2}$ & 0.00951 & 0.01417 \\
\hline
\end{tabular}




\subsection{FOR LATERAL INSTABILITY OF BEAMS}

\subsubsection{Rayleigh-Ritz method}

The variational Rayleigh-Ritz method enables to determine closed-form solution of instability problems. From the principle of stationary potential energy, equation (61), the equilibrium configuration or the instability load of a structural member can be determined in a slightly displaced configuration.

$$
\frac{\partial \Pi}{\partial \alpha_{i}}=0 ; i=1,2, \ldots, n
$$

An essential advantage of the Rayleigh-Ritz method is that the approximated functions adopted to solve the static problems are only required to satisfy the essential boundary conditions. Thus, a proper shape for the beam must be assumed as a function of degrees of freedom $\alpha_{i}$ and then substituted in the energy functional $\Pi$, which is minimized. The derivatives obtained from equation (61) result in a system of linear equations.

$$
a_{i j} \alpha_{j}=0, i, j=1,2, \ldots, n
$$

Then, to determine the critical load, assuming no trivial solution $\alpha_{j}=0$ for equation (62), the following condition must be guaranteed

$$
\operatorname{det}\left(a_{i j}\right)=0
$$

Depending on the adopted approximations for displacement and rotation, equation (63) can results in a polynomial, in which the small root represents the critical load.

\subsubsection{Instability of beams lifted by cables}

During lifting, the absence of any constraint against rotation at the lifting points makes the equilibrium unstable for any small lateral eccentricity de even when only the selfweight acts, Figure 125 (a). Thus, the equilibrium is only possible for a slightly rotated configuration, assuming rigid body rotation, with the center of mass $Q$ passing through the axis of rotation as shown in Figure 125 (b). 


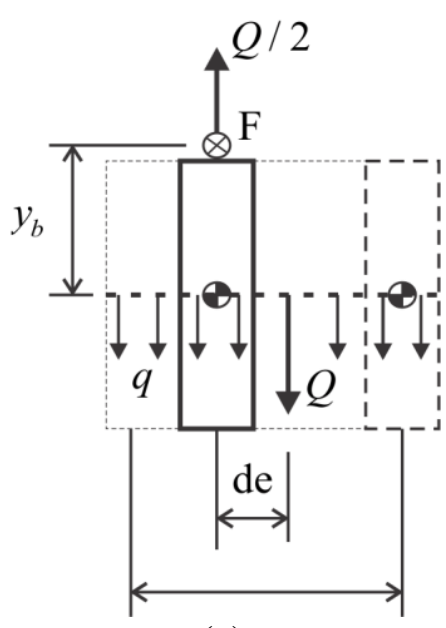

(a)

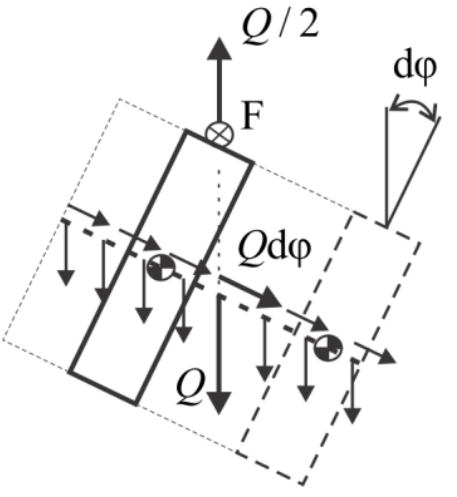

(b)

Figure 125 - Mechanical behavior of lifted beams

However, the equilibrium is only reached if the lateral component of the self-weight $Q \times d \varphi$ is small. Thus, depending on the level of this component, a new eccentricity can arise, which corresponds to an unstable equilibrium again. This process will occur until the equilibrium of the element. Therefore, for small displacements, such equilibrium configuration is considered at the present work as the bifurcation point, where the eccentricity de approaches to zero and the beam has a small enough lateral component of the self-weight $Q \times d \varphi$.

Thus, the beam presents a stable behavior when the position of the center of mass of the applied loads coincides with the axis of rotation, which passes through the lifting points. Figure 126 presents a plan view of the rotated beam with the origin of the $\mathrm{x}$ and $\mathrm{z}$-axis at the lifting point.

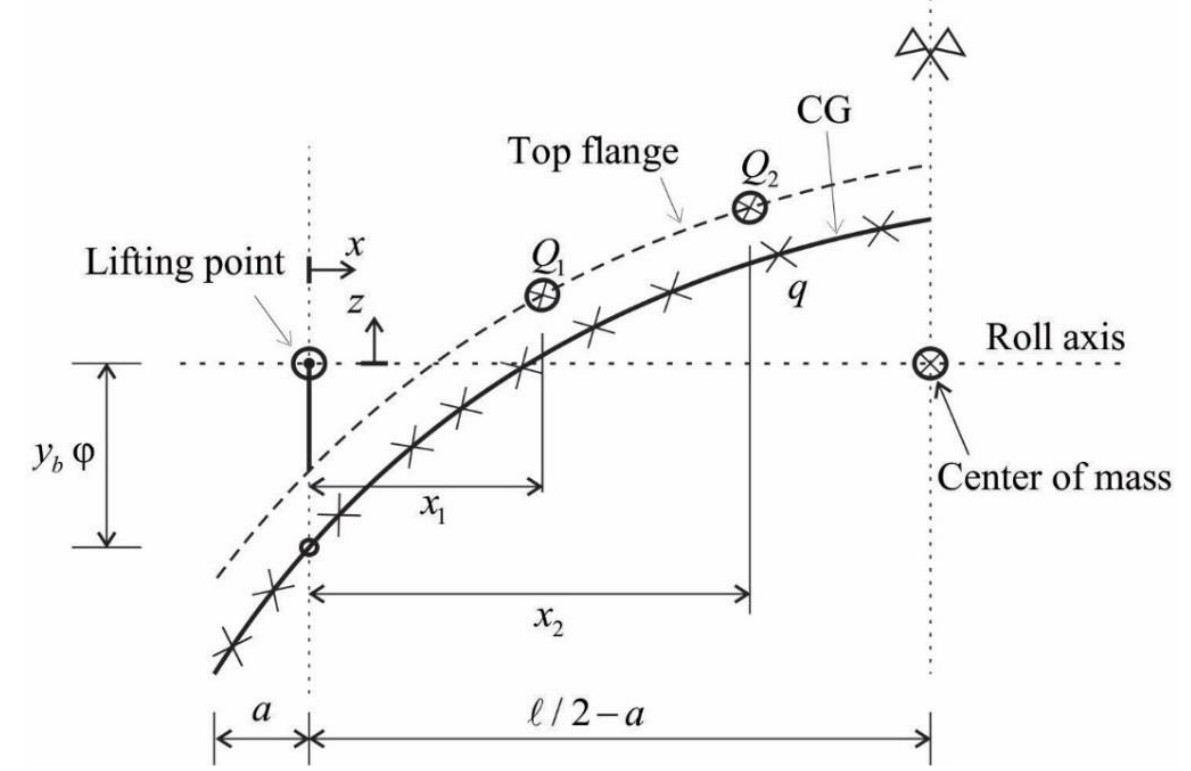

Figure 126 - Plan view of the slightly rotated beam at the equilibrium position 
The position of the center of mass is obtained by

$$
\begin{aligned}
& z^{\prime}=\frac{\int_{-a}^{\ell-a} q z_{q} d x+\Sigma z_{Q_{i}} Q_{i}}{\int_{-a}^{\ell-a} q d x+\Sigma Q_{i}} \\
& z_{q}=u(x)+y_{q} \varphi \\
& z_{Q_{i}}=u\left(x_{i}\right)+y_{Q_{i}} \varphi
\end{aligned}
$$

Where $Q_{q}$ is the resultant of the self-weight and $z_{q}$ its coordinate and $Q_{i}$ is an applied load at any point and $z_{Q i}$ its coordinate, $y_{q}$ and $y_{Q i}$ are the height of the applied loads relative to the centroid, which is positive when above the centroid. Thus, $z$ ' must be zero to guarantee the equilibrium that enables to determine one more constant $\alpha_{i}$ of the displacement function. When only self-weight acts, the center of mass is

$$
z^{\prime}=\frac{\int_{-a}^{\ell-a} z_{q} d x}{\ell}
$$

In which $\ell$ is the span of the beam, $a$ is the overhang length. Figure 126 presents onehalf of the plan view of the static scheme.

During construction, it is a common practice to lift beams with one crane with inclined cables. In this cases, a component of the reaction of the cables acts axially to the beam section and consequently a constant moment due to the eccentricity of this load, as shown in Figure 127.

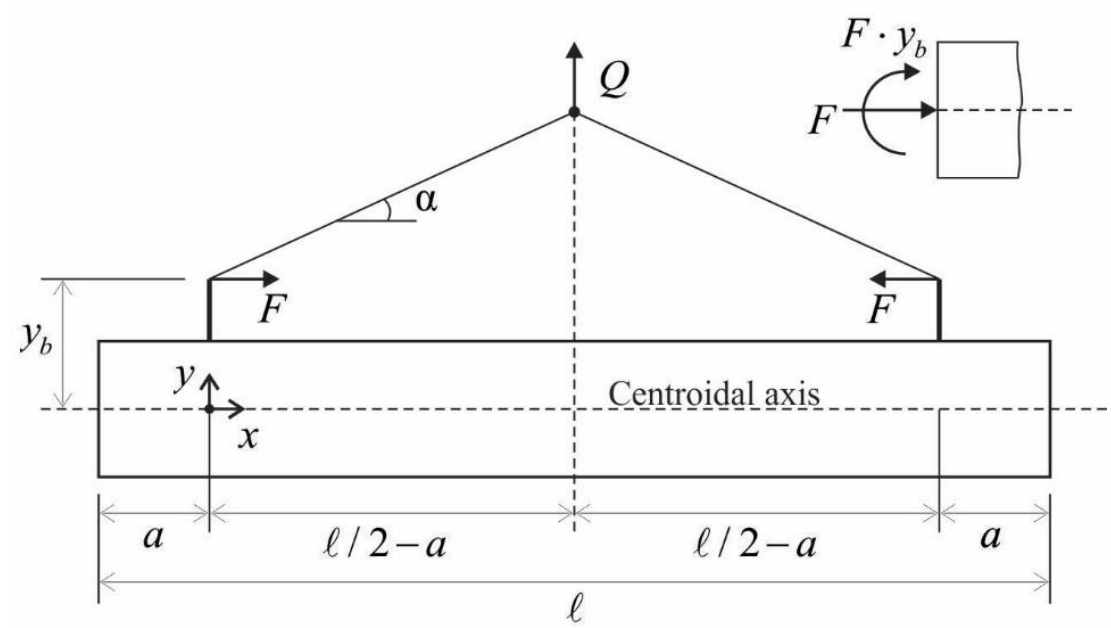

Figure 127 - Concrete beam lifted by inclined cables

The axial load $F=Q / 2(\tan \alpha)$ must be considered in instability analysis. The eccentricity of this force is $y_{b}$. As discussed previously, the beam is assumed to rotate as a 
rigid body due to the support condition. Thus the approximate solution equation for the rotation is

$$
\varphi=\alpha_{1}
$$

For lateral displacements to evaluate the convergence of the method. Three kinematic boundary conditions allow rewriting the displacement function to reduce the number of linear equations in equation (62). As the Rayleigh-Ritz method do not require that all boundary condition be satisfied, only the vertical twist considered as zero at midspan is imposed, equations (68). Equation (69) represents a necessary condition for equilibrium that is the center of mass must result in the roll axis passing through the lifting points. Equation (70) represents the displacement function, or Ritz solution, where $\alpha_{j}$ are constants and $\phi_{j}$ are admissible functions.

$$
\begin{aligned}
& \left.\frac{d u}{d x}\right|_{x=\ell / 2-a}=0 \\
& z^{\prime}=0 \\
& u(x)=\alpha_{j} \phi_{j}
\end{aligned}
$$

A five-order polynomial was adopted to represent the lateral displacements. Assuming that the beam suffers only flexural strains and no torsion strains, the internal energy correspondent to St. Venant and warping torsions, presented in equation (71), will vanish from the internal energy functional.

$$
U=\int_{-a}^{\ell / 2-a}\left\{E I_{z} u^{\prime \prime 2}+G J \varphi^{\prime 2}+E I_{\omega} \varphi^{\prime 2}\right\} d x
$$

Where $E I_{z}, G J, E I_{\omega}$ are the lateral flexural rigidity, torsional and warping rigidities. For the work done by the external loads, the load heigh relative to the centroid is considered. During buckling, a load $P$ applied at any position of the span, and at a distance $y_{q}$ from the centroid causes a displacement $d$, which represents the decrease in the potential energy due to the lateral flexural strains, see Figure 128. Thus, for the determination of the displacement $d$, the geometric quantities $b$ and $c$, shown in Figure 128, must be determined. 

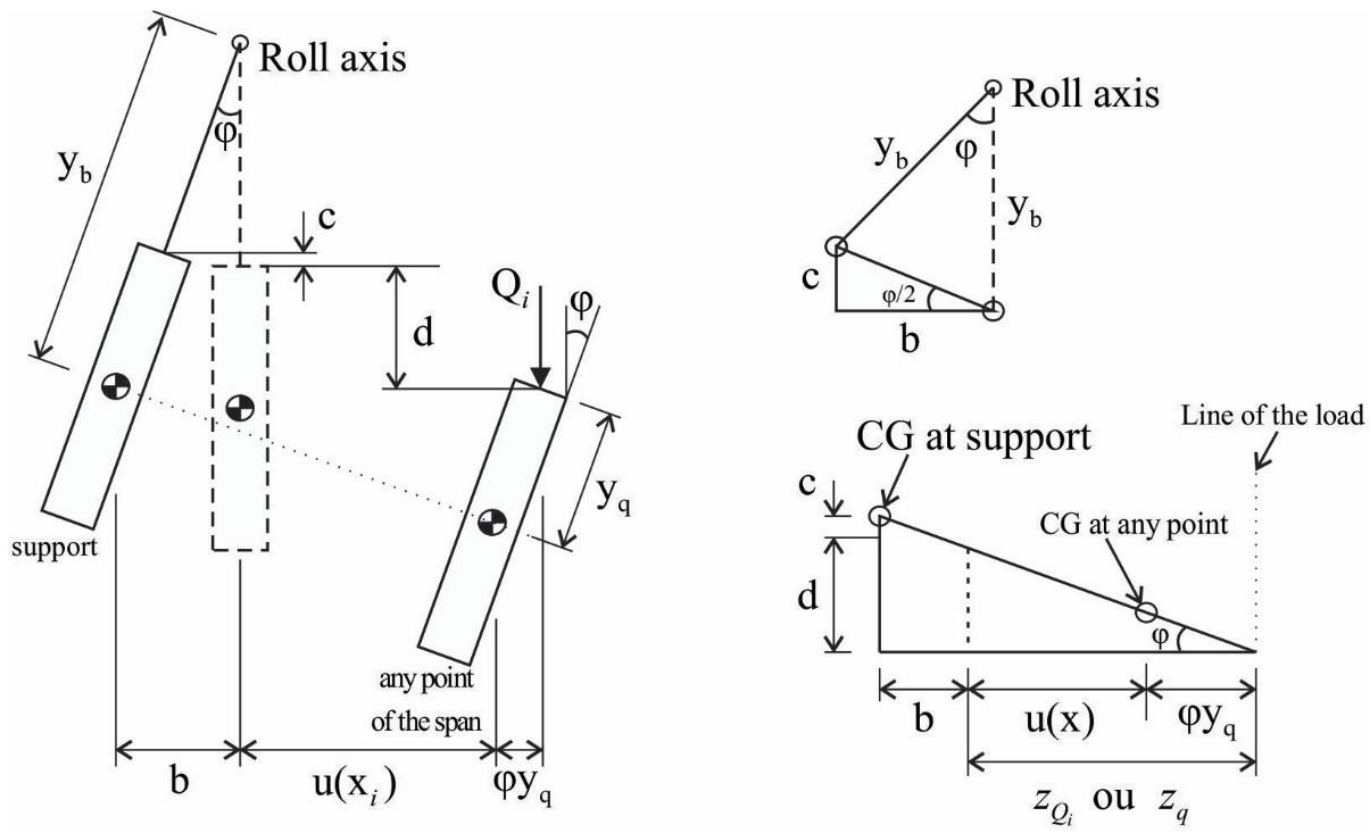

Figure 128 - Geometrical quantities at stable equilibrium

Considering that the beam undergoes rigid body rotation, the quantities $b$ and $c$ and the displacement $d$ are determined by

$$
\begin{aligned}
& b=y_{b} \varphi \\
& c=\frac{y_{b} \varphi^{2}}{2} \\
& d=z_{q} \varphi+\frac{y_{b} \varphi^{2}}{2}
\end{aligned}
$$

Where $y_{b}$ is the distance of the roll axis from the centroid. These expressions are valid for any loading. Thus, the work done during buckling by the distributed loading $q$ and concentrated loads $Q_{i}$ is determined by

$$
V_{1}=\int_{-a}^{\ell / 2-a} q\left(2 z_{q} \varphi+y_{b} \varphi^{2}\right) d x+\Sigma Q_{i}\left(z_{Q_{i}} \varphi+\frac{y_{b} \varphi^{2}}{2}\right)
$$

Where

$$
\begin{aligned}
& z_{q}=u(x)+y_{q} \varphi \\
& z_{Q_{i}}=u\left(x_{i}\right)+y_{Q_{i}} \varphi
\end{aligned}
$$

$y_{q}$ is the height of the distributed loading and $y_{Q i}$ for the concentrated loads. The work done by the axial component of the reaction force of the cables is determined by

$$
V_{2}=\int_{0}^{\ell / 2-a} F u^{\prime 2} d x+\int_{0}^{\ell / 2-a} F y_{b} u^{\prime} \varphi^{\prime} d x
$$


As the approximation for rotations is constant, the second term of this equation vanishes. Furthermore, the rigid body rotation is accounted as work done by the external loads by

$$
V_{3}=M_{R, P} \varphi+M_{R, q} \varphi=P z_{Q i} \varphi+\int_{-a}^{\ell-a} q z_{q} \varphi d x
$$

Therefore, the total energy of the system is

$$
\Pi=U-V_{1}-V_{2}-V_{3}
$$

By minimizing the equation of energy, the stationary condition presented in equation (61) enables to obtain the buckling load of beams lifted by inclined or straight cables with or without overhangs.

$$
Q_{c r}=\frac{240 E I_{z} \ell\left(6720 a^{6}-3360 a^{5} \ell+3920 a^{4} \ell-2800 a^{3} \ell+924 a^{2} \ell^{4}-154 a \ell^{5}+11 \ell^{6}\right)}{(2 a-\ell)^{10}\left[\frac{7 y_{b}}{y_{q}{ }^{2}}-\frac{10520}{(2 a-\ell) \tan \alpha}\right]}
$$

Where $\alpha$ is the angle of inclination of the cables, $\ell$ is the total span length, $a$ is the overhang length, $E I_{z}$ is the lateral flexural stiffness, $y_{q}$ and $y_{b}$ are the load height relative to centroid and the distance between the centroid and the lifting point, respectively. The equation proposed by (SOUTHWELL, 1932) is utilized to consider the effect of initial rotations which enables to determine the cracking load or failure load of imperfect beams.

\subsubsection{Instability of beams supported by bearing pads}

Prestressed beams are usually centrally prestressed relative to lateral flexural deflections although eccentrically for in-plane bending. As discussed by (BAŽANT; CEDOLIN, 1991), the prestress force does not affect buckling for centrally prestressed elements, which also occur for lateral stability of beams. The axial force due to prestress tries to buckle the beam sideways, but the tensioned cables attempt to keep the beam straight, see Figure 129. Thus, the forces are self-balanced. To demonstrate this assumption, the energy functional $\Pi$ accounts for the prestress force and its effect. 


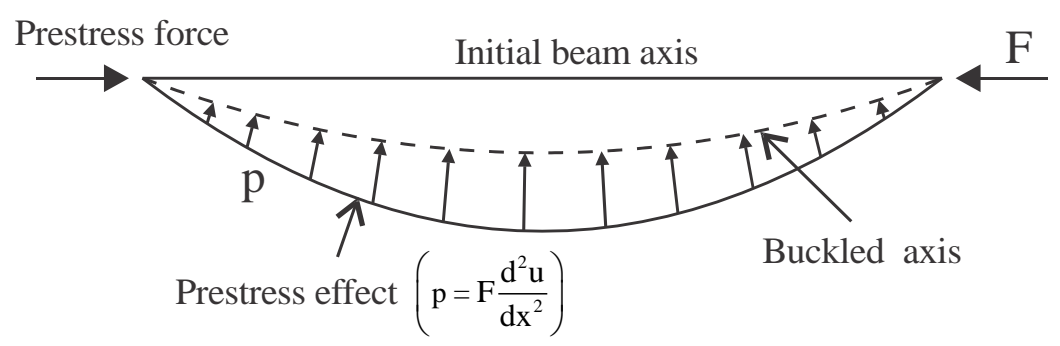

Figure 129 - Top view of the laterally buckled prestressed beam, adapted from (BAŽANT;

CEDOLIN, 1991)

From the hypothesis that the beam suffers only flexural strains due to lateral bending, the energy correspondent to torsion strain is neglected, and therefore the strain energy stored during rollover of precast beams are flexural strain energy, the energy of the spring supports and the effect of prestressing, equation (71).

$$
U=\int_{0}^{\ell} \frac{E I_{y}}{2}\left(\frac{d^{2} u}{d x^{2}}\right)^{2} d x+k_{\theta} \varphi^{2}+\int_{0}^{\ell} \frac{F}{2} u \frac{d^{2} u}{d x^{2}} d x
$$

The parameter $k_{\theta}$ represents the stiffness of the pad against rotation of the beam around its longitudinal axis. $F$ is the prestress force.

As discussed, the lateral displacement is a function of longitudinal axis rotation. From this, the lateral moment $M_{y}^{\prime}$ of the beam at instability is a function of the strong axis bending moment $M_{z}$ of the undisplaced beam. Figure 130 presents the relations between the internal moments of the displaced and undisplaced beam and the geometrical quantities involved in the current formulation.

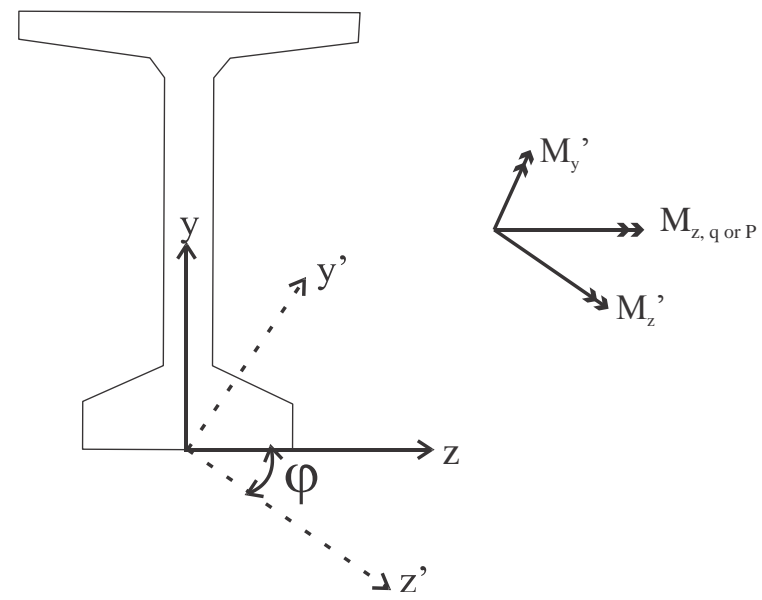

(a)

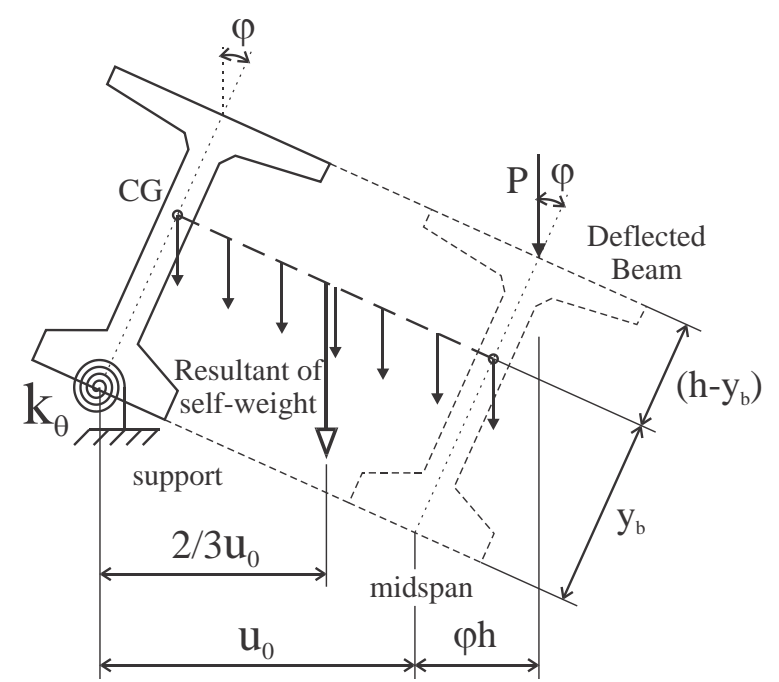

(b)

Figure 130 - (a) Components of $M_{z}$ in the z' and y' axis and (b) geometrical variables of the proposed analytical equation 
The relations presented in Figure 130 (a) enables to write the weak axis curvature of the beam at instability as a function of the beam rigid body rotation $\sin \varphi \cong \varphi$ and the strong axis bending moment $M_{z}$, (CHAJES, 1974), equation (80).

$$
\frac{d^{2} u}{d x^{2}}=\frac{M_{z} \varphi}{E I_{y}}
$$

Where $M_{z}$ is the internal moment of the toppled beam acting about the z-axis of the untoppled beam. The load cases studied are presented in equations (81), (82) and (83).

$$
\begin{aligned}
& M_{z, P}=\frac{P x}{2}, \text { central point load } \\
& M_{z, w}=\frac{q x}{2}(\ell-x), \text { uniformly distributed load } \\
& M_{z, P P}=P x-P\left\langle x-\frac{1}{3} \ell\right\rangle, \text { symmetrical two-point loads }
\end{aligned}
$$

Where the Macauly bracket \langle\rangle represents the Heaviside function which is zero for negative values. Figure 131 shows the static schemes for the studied loading cases.
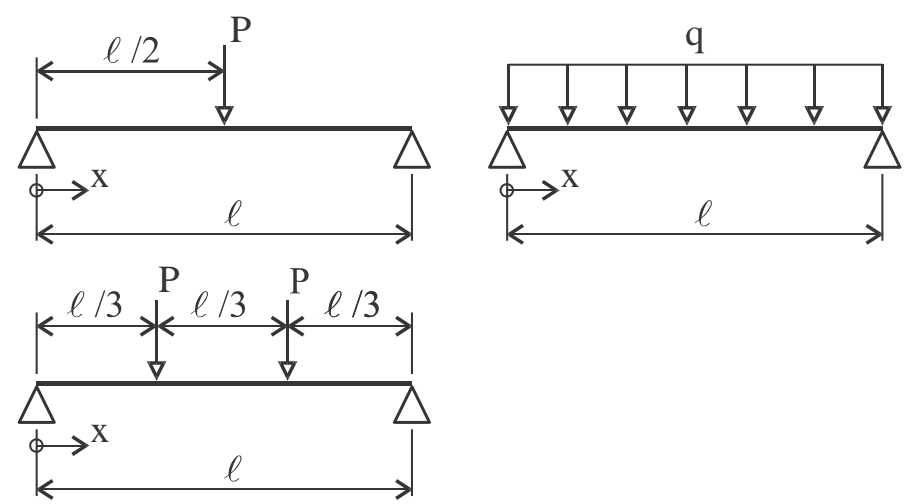

Figure 131 - Static schemes of the load cases studied

As already discussed, precast beams without restraints at supports suffer small torsional strains, and therefore a more significant portion of the rotation is due to the rigid body movement. Thus, for the proposed solution, the rotation is taken as constant throughout the span, equation (67) and the approximation for lateral deflection is assumed to be given by a quadratic polynomial, equation (69).

$$
\begin{aligned}
& \varphi=\alpha_{1} \\
& u=\alpha_{2}+\alpha_{3} x+\alpha_{4} x^{2}
\end{aligned}
$$


Therefore, the equilibrium occurs when the energy functional is minimized relative to the presented degrees of freedom $\alpha_{i}$.

The three parts $V_{1}, V_{2}$ and $V_{3}$ compose the potential energy due to external loads in the current study. The first term $V_{1}$ refers to the work done by the external loads that cause the vertical displacement associated with the lateral flexural displacement at rollover. Furthermore, $V_{1}$ accounts for the prestressing load that tends to buckle the beam. Thus, $V_{1}$ is written as a function of internal moments, equations (81) and (82), as in (TIMOSHENKO; GERE, 1988) and (TRAHAIR, 2016), and the prestress force.

$$
V_{1}=-\int_{0}^{\ell} M_{z} \varphi \frac{d^{2} u}{d x^{2}} d x-\frac{F}{2} \int_{0}^{\ell}\left(\frac{d u}{d x}\right)^{2} d x=-\int_{0}^{\ell} \frac{\left(M_{z, P}+M_{z, q}\right)^{2} \varphi^{2}}{E I_{y}} d x-\frac{F}{2} \int_{0}^{\ell}\left(\frac{d u}{d x}\right)^{2} d x
$$

Note that, the terms $1 / 2 \int_{0}^{\ell} q y_{q} \varphi^{2} d x$ and $1 / 2 P y_{q} \varphi^{2}$, which account for the load height relative to the shear center, (TIMOSHENKO; GERE, 1988) and (TRAHAIR, 2016), do not appear in equation (86). As adopted, the beams do not suffer torsional strain during the rollover, and consequently, the load position displaced from the shear center do not cause torque which activates the uniform and nonuniform torsion rigidities. Similar expressions will appear in the terms $V_{2}$ and $V_{3}$ that are relative to rigid body rotation.

As mentioned, the other two terms $V_{2}$ and $V_{3}$ correspond to the rigid body rotation caused by the vertical loads. As discussed in other papers (MAST, 1993), (BURGOYNE; STRATFORD, 2001) and (PLAUT; MOEN, 2014), the axis of rotation passes through the bearing pads. Thus, $V_{2}$ represents the work done by the load $P$ applied at midspan, equation (87), and $V_{3}$ the work done by the self-weight $q$, equation (88).

$$
\begin{aligned}
& V_{2}=-M_{R, P} \varphi=-\left(P u_{0} \varphi+P h \varphi^{2}\right) \\
& V_{3}=-M_{R, q} \varphi=-\left(\frac{2 u_{0} q \ell \varphi}{3}+y_{b} q \ell \varphi^{2}\right)
\end{aligned}
$$

As can be seen, the rollover moments account for the effect of the load position. In equations (87) and (88), $u_{0}$ is the lateral displacement of the beam at midspan, $\boldsymbol{h}$ is height, $y_{b}$ is the height of the centroid from bottom face and $\ell$ is the span of the beam. In the 
potential energy $V_{3}$, the lateral displacement $u_{0}$ is multiplied by $2 / 3$ to account for the position of the resultant of the self-weight with relation to the beam axis of rotation, (MAST, 1993) and (PLAUT; MOEN, 2014), see Figure 130.

The displacement $u_{0}$ at midspan occurs due to lateral flexural strain and can be determined by equation (89) as a function of the rigid body twist.

$$
u_{0}=\int_{0}^{\ell / 2} \frac{M_{z} \varphi}{E I_{y}} x d x=\int_{0}^{\ell / 2} \frac{\left(M_{z, P}+M_{z, q}\right) \varphi}{E I_{y}} x d x
$$

Therefore, the total energy of the system is represented by equation (90).

$$
\Pi=U+V_{1}+V_{2}+V_{3}
$$

From the stationary condition presented in equation (61) and considering the hypothesis of small displacements and rotations (due to the large spans of precast beams), the equation (90) is minimized to obtain the equation (91), which represents the equilibrium condition.

$$
\alpha_{1}\left(2 k_{\theta}-2 P h-2 \ell q y_{b}-\frac{74 \ell^{5} q^{2}+155 P \ell^{4} q+540 P^{2} \ell^{3}}{2880 E I_{y}}\right)=0
$$

Where, if $\alpha_{1}$ is zero (trivial solution), equation (91) does not represent the instability configuration. Thus, for the nontrivial solution, the terms in parentheses must be zero, which enables to determine the critical rollover load $P_{c r}$ as the smaller positive root of the expression. Equation (92) presents the critical load for simply supported beams with a concentrated load at midspan. The equation considers the beam self-weight simultaneously.

$$
P_{c r}=-\frac{31 q \ell}{216}-\frac{16 E I_{y}}{3 \ell^{3}}\left[h-\sqrt{\frac{3}{8 E I_{y}}\left(k_{\theta} \ell^{3}-q y_{b} \ell^{4}\right)+\ell^{2}+\frac{31 q h \ell^{4}}{576 E I_{y}}-\frac{0.004093 q^{2} \ell^{8}}{E I_{y}}}\right]
$$

Note that the prestress force does not appear in equation (92), because the potential energy due prestress and the developed prestress effect cancel each other out for an approximated solution like a quadratic polynomial. Thus, in the bifurcation problem, centrally prestressing does not affect the solution, (BAŽANT; CEDOLIN, 1991).

If only the self-weight acts, as is common in construction stages, it is interesting to determine the critical self-weight $q_{c r}$ for beam rollover. For this, cancel the parts correspondent to the load $P$ from equation (91) and the smaller root for $q$ corresponds to the self-weight that causes rollover of the beam, equation (93). 


$$
q_{c r}=\frac{24}{17} \frac{\sqrt{185 E I_{y} k_{\theta} \ell^{3}+3600 y_{b}^{2}\left(E I_{y}\right)^{2}}-60 y_{b} E I_{y}}{\ell^{4}}
$$

For the case of beams with symmetrical two-point loads, the potential energy due to external loads $V_{1}$, equation (86), is rewritten as a function of the moment $M_{z, P P}$ expressed in equation (83). $V_{2}$ represents now the potential energy correspondent to the third-point concentrated loads that cause rigid body rotation, equation (94). Assuming a parabolic form for the lateral deflection, therefore, the displacement at the points of load $(\ell / 3)$ relative to the axis of rotation is $u_{0}$ multiplied by $8 / 9$.

$$
V_{2}=-M_{R, P P} \varphi=-2\left(\frac{8}{9} P u_{0} \varphi+P h \varphi^{2}\right)
$$

Thus, equation (95) presents the minimized functional. The solution for the critical load $P_{c r}$ is the positive root of the given equation.

$$
\begin{aligned}
& \alpha_{1}\left(2 k_{\theta}-\frac{P^{2}}{E I_{y}} A-\frac{P}{E I_{y}} B-\frac{37}{1440} \frac{\ell^{5} q^{2}}{E I_{y}}-2 q \ell y_{b}\right)=0 \\
& A=\frac{79}{108} \ell^{3}-\frac{283}{162} \ell^{2}+\ell \\
& B=\frac{11}{108} \ell^{4} q-\frac{5}{54} \ell^{3} q+4 h E I_{y}
\end{aligned}
$$

Besides the results of experimental researches (CONSOLAZIO; HAMILTON, 2012) and (HURFF; KAHN, 2012b) did not detect cracking before rollover of the precast beams, the nonlinear behavior of concrete can be considered by the penalization of flexural and torsional rigidities. In this case, the rollover load which causes instability is now calculated with an effective flexural rigidity. Such an approach is named inelastic buckling. In several researches (HANSELL; WINTER, 1959), (HANSELL; WINTER, 1959), (SANT; BLETZACKER, 1961) (REVATHI; MENON, 2006), the authors propose an approximate solution for these effective rigidities according to experimental evidence. 


\section{RESULTS}

\subsection{ANALYTICAL}

\subsubsection{UHPFRC beams lifted by cables}

Figure 132 presents the comparison between the experimental and analytical of loadrotation curves, all starting from zero. The analytical simulations accounted for three values of initial rotation, including the average of 1.67 degrees. The average width of the beam was considered. Also, the differences in the experimental peak load from the analytical solutions are highlighted.

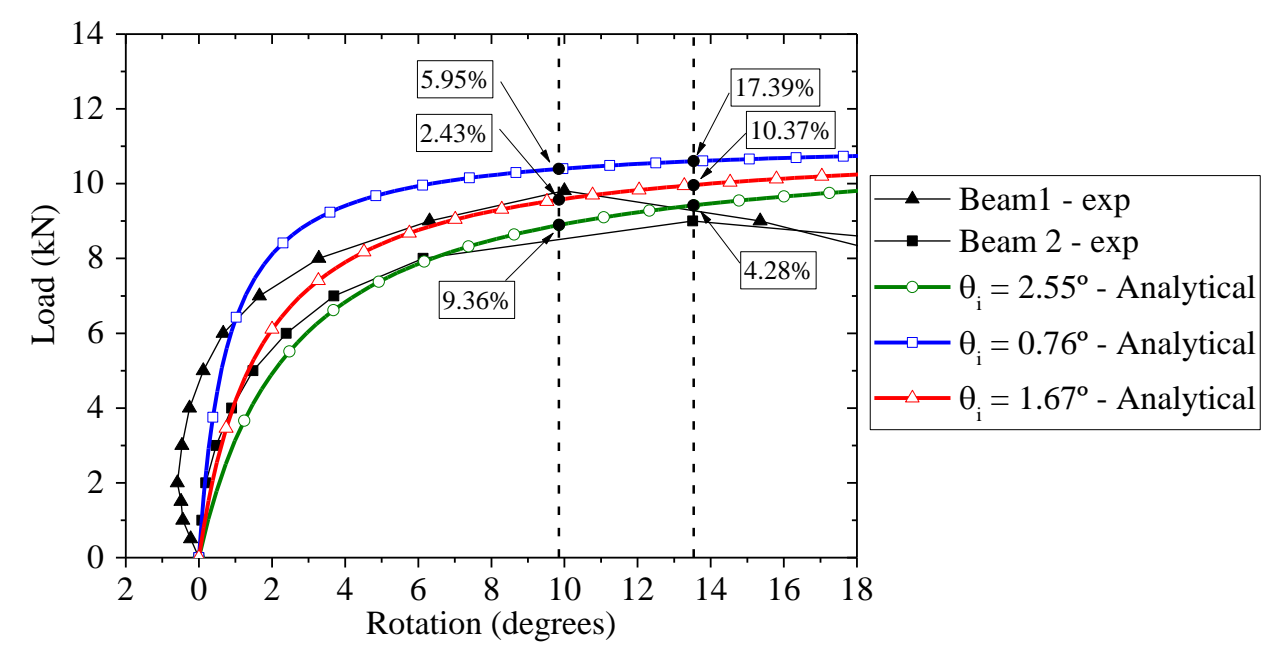

Figure 132 - Comparison of experimental and analytical results

The higher difference in prediction was $17.39 \%$. The analytical solution considering the average value of initial rotation presented differences of $2.4 \%$ and $10.37 \%$ for the peak load of the beam 1 and beam 2, respectively. Thus, the proposed solution predicts with great accuracy the experimental results of UHPFRC beams. The same problem was simulated for a beam with a modulus of elasticity of an ordinary concrete of $30 \mathrm{GPa}$. The imperfection angle was 2.55 degrees, and the cables are inclined 47 degrees and positioned $0.5 \mathrm{~m}$ from the ends. The buckling load was $7.61 \mathrm{kN}$ considering the limit rotation of 12.5 degrees, which is $32 \%$ smaller than the UHPFRC beam at the same conditions. The higher modulus of elasticity makes the UHPFRC beams less favorable to present large displacements and consequently less prone to lateral instability. 


\subsubsection{Concrete beams supported by bearing pads}

The experimental results of (HURFF; KAHN, 2012b) and (CONSOLAZIO; HAMILTON, 2012) are the references to compare to the results of the proposed solutions, equations (92) and (95). The first beam is a simple supported PCI BT-54 I-section subjected to a central point load tested by (HURFF; KAHN, 2012b) and the second is an almost rectangular section subjected to symmetrical two point loads, (CONSOLAZIO; HAMILTON, 2012). Table 16 presents the geometrical and mechanical properties of the studied beams. Furthermore, the initial lateral imperfection, as well as the displacement at rollover, are presented.

Table 16 - Geometrical and mechanical properties of the beams

\begin{tabular}{|c|c|c|}
\hline Properties & $\begin{array}{c}\text { (HURFF; KAHN, } \\
\text { 2012b) }\end{array}$ & $\begin{array}{l}\text { (CONSOLAZIO; HAMILTON, } \\
\text { 2012) }\end{array}$ \\
\hline Span $(\mathrm{m})$ & 30.50 & 30.50 \\
\hline Height (mm) & 1372 & 1981 \\
\hline Area $\left(\mathrm{mm}^{2}\right)$ & 425642 & 261290 \\
\hline Height of centroid $y_{b}(\mathrm{~mm})$ & 703 & 975 \\
\hline $\begin{array}{l}\text { Weak axis moment of inertia } \\
\qquad I_{y}\left(\mathrm{~mm}^{4}\right)\end{array}$ & $1.55 \times 10^{10}$ & $4.73 \times 10^{8}$ \\
\hline $\begin{array}{l}\text { Initial lateral displacement } \\
(\mathrm{mm})\end{array}$ & 43.50 & 70.30 \\
\hline $\begin{array}{l}\text { Limit lateral displacement } \\
\text { (mm) }\end{array}$ & 95.20 & 168.30 \\
\hline Modulus of elasticity (GPa) & 30.82 & 35.85 \\
\hline
\end{tabular}

Steel reinforced elastomeric bearing pads are the supports of both beams. Table 17 shows the geometrical and mechanical properties of the pads.

Table 17 - Properties of the bearing pads

\begin{tabular}{ccc}
\hline & (HURFF; KAHN, & (CONSOLAZIO; HAMILTON, \\
& $2012 \mathrm{~b})$ & $2012)$ \\
\hline Length, L (mm) & 610.0 & 609.6 \\
Width, W (mm) & 360.0 & 274.0 \\
Height, H (mm) & 73.0 & 48.4 \\
Internal elastomer thickness & 15.0 & 12.7 \\
(mm) & 7.5 & 6.4 \\
External elastomer thickness & 4 & 3 \\
Number of internal plates & 0.67 & 0.76 \\
Shear modulus $G_{p}(\mathrm{MPa})$ & & \\
\hline
\end{tabular}


The contact between beam and pad is critical for rollover stability because the liftoff effect reduces the elastic rotational stiffness of the bearing pad due to gradual loss of contact area. This phenomenon occurs mainly during the toppling of the beam and has a nonlinear relationship between moment and rotation, (CONSOLAZIO; HAMILTON, 2012). Also, lift-off is the main reason for earlier rollover failure, even before any inelastic behavior of concrete, (HURFF; KAHN, 2012b) and (CONSOLAZIO; HAMILTON, 2012). Thus, to estimate the nonlinear rotational stiffness of the pads at the moment of rollover, a simplified numerical model based on finite element method was utilized, which is similar to the models proposed in other studies, (HARPER; CONSOLAZIO, 2013) and (LEE et al., 2017b). The model consists of a rigid grillage representing the mid-surface of the pad with springs attached to the grillage interception nodes. Furthermore, the springs are not capable of supporting tensile forces which account for the possible loss of contact between pad and beam and have a nonlinear compressive force versus displacement relation.

The horizontal mid-surface of the pad consists of 72 parts $(50.8 \mathrm{~mm} \times 50.8 \mathrm{~mm})$, and in the center of each portion, a spring with nonlinear behavior was attached receiving the tributary compressive stiffness of the pad, Figure 133. Figure 134 shows the comparison between the experimental and numerical compressive force versus axial displacement relationship of the pad.

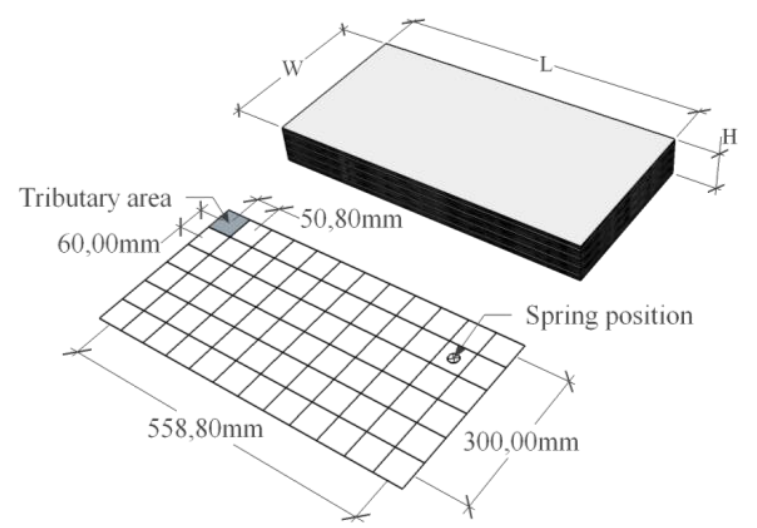

Figure 133 - Pad divisions to determine the tributary spring stiffness

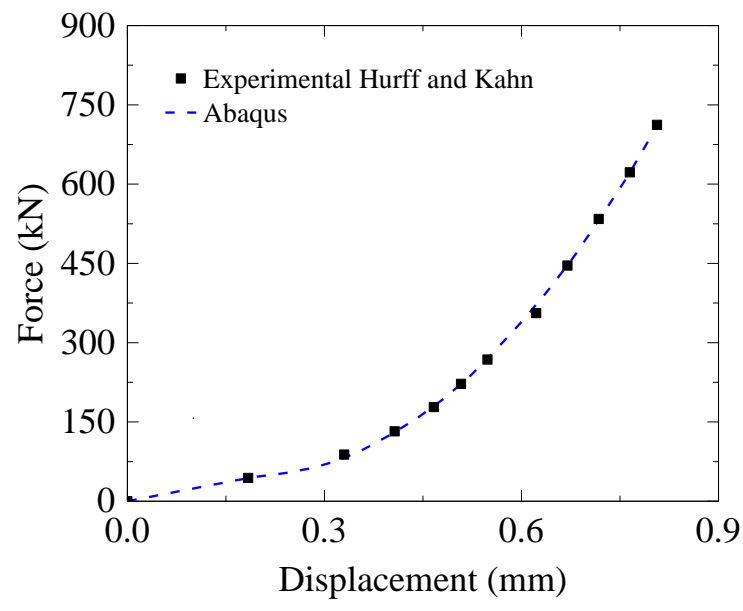

Figure 134 - Numerical and experimental axial response of elastomeric bearing pad

As expected, the axial response of the grillage model represented precisely the experimental curve, as shown in Figure 134. To obtain the moment versus rotation relation of the pad, firstly the beam self-weight is applied which adjust the stiffness of the pad as in real conditions. Then, an applied rotation simulates the beam toppling with possible lift-off of the pad, Figure 135. (HURFF; KAHN, 2012b) reported that at rollover the beam rotated 
$0.09 \mathrm{rad}$ at supports, which is highlighted in Figure 135 and the correspondent secant stiffness. Figure 136 presents the comparison of the experimental results of (CONSOLAZIO; HAMILTON, 2012) and the numerical moment versus rotation relationship obtained with the current methodology. The last authors did not report the rotation at supports at moment of rollover.

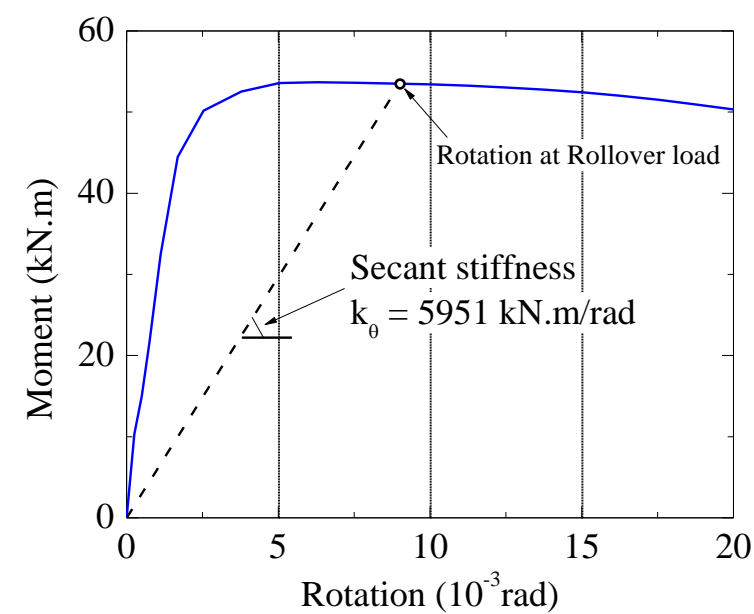

Figure 135 - Numerical moment versus rotation behavior of the pad tested in (HURFF;

KAHN, 2012b)

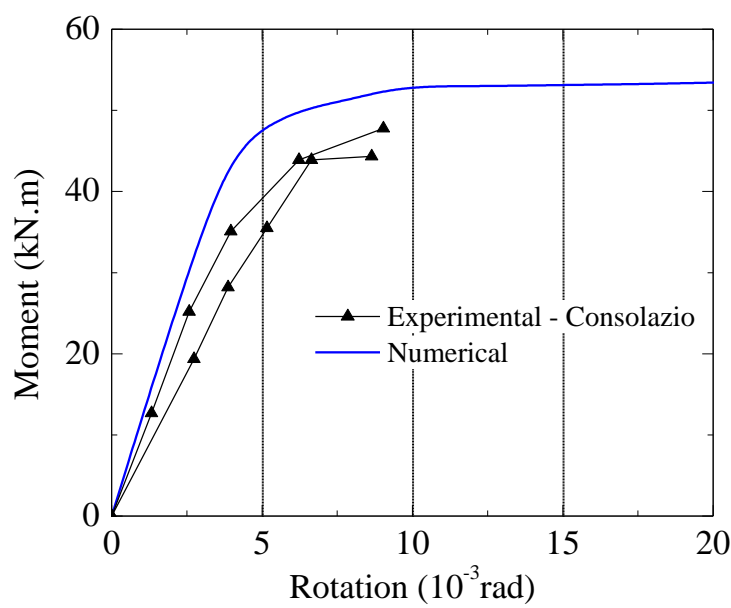

Figure 136 - Comparison of experimental and numerical moment versus rotation behavior of the pad tested in (CONSOLAZIO;

HAMILTON, 2012)

Due to the lift-off effect, the moment versus rotation response is nonlinear, as shown in the figures. The initial tangent stiffness obtained numerically for the pad of (HURFF; KAHN, 2012b) was $27490 \mathrm{kN} . \mathrm{m} / \mathrm{rad}$ that is $10 \%$ greater than the experimental result. The initial tangent for the pad of (CONSOLAZIO; HAMILTON, 2012) was $11612 \mathrm{kN} . \mathrm{m} / \mathrm{rad}$ which is $18 \%$ higher than the experimental result.

The secant stiffness presented in Figure 135 was utilized to calculate the rollover load of the first beam with the proposed equation (92). The Southwell equation allowed the determination of the limit load. The density considered for reinforced concrete was 25 $\mathrm{kN} / \mathrm{m}^{3}$. Thus the I-section beam has a self-weight of $11.39 \mathrm{kN} / \mathrm{m}$, and the rectangular beam has $6.53 \mathrm{kN} / \mathrm{m}$.

As mentioned, the Southwell equation also enables to determine the nonlinear force versus lateral displacement by incrementally increasing the displacement $x=\delta_{\text {lim }}$ until reach 
the limit displacement. Figure 137 presents the experimental force versus lateral displacement curve of the PCI BT-54 beam compared to the proposed solution combined with the Southwell equation considering different values of $n$.

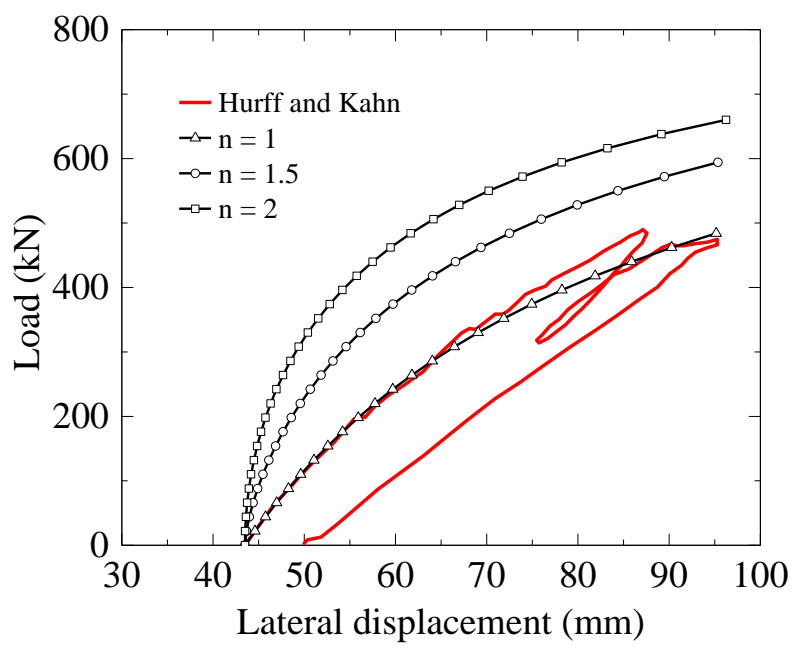

Figure 137 - Comparison of experimental results and the proposed analytical solution

For the constant $n$ taken equal one, Figure 137, the Southwell equation predicted well the load-bearing capacity and the whole load versus lateral displacement curve. The proposed solution presented an error in the prediction of the limit rollover load of $4.54 \%$.

The experimental result of (HURFF; KAHN, 2012b) is compared to previously proposed equations, (MAST, 1993), (BURGOYNE; STRATFORD, 2001) and (LEE, 2017), to show that the proposed equation present an advance in this research field, t. As these equations account for a load acting on the centroid, the quantity $y_{b}$, which is the height of the centroid, was changed by $h$, the height of the beam, trying to account for load height. Besides, the critical load of these solutions was subtracted by a concentrated load relative to self-weight $q \times \ell$, which diminishes the critical load and makes the solution more critical.

Table 18 - Comparison of analytical results for the beam tested by (HURFF; KAHN, 2012b)

\begin{tabular}{ccc}
\hline Authors & Rollover load (kN) & Difference (\%) \\
\hline Experimental & 463 & - \\
(MAST, 1993) & 2063 & 345.6 \\
(BURGOYNE; STRATFORD, 2001) & 1833 & 295.9 \\
(LEE, 2017) & 1428 & 208.4 \\
Proposed & 484 & 4.5 \\
\hline
\end{tabular}

It can be seen from the table that even with some adjustments, the equations of (MAST, 1993), (BURGOYNE; STRATFORD, 2001), (LEE, 2017) are not applicable to predict the behavior of beams with different loading conditions from the self-weight. 
For the beam tested by (CONSOLAZIO; HAMILTON, 2012), a sensitivity analysis verified the rollover stability. Firstly, $n$ received value equal one and $k_{\theta}$ varied from the elastic value to a quarter of this initial value, Figure 138, to represent the possible occurrence of the lift-off. After, $k_{\theta}$ was maintained as the elastic value and $n$ took $1,1.5$ and 2, Figure 139.

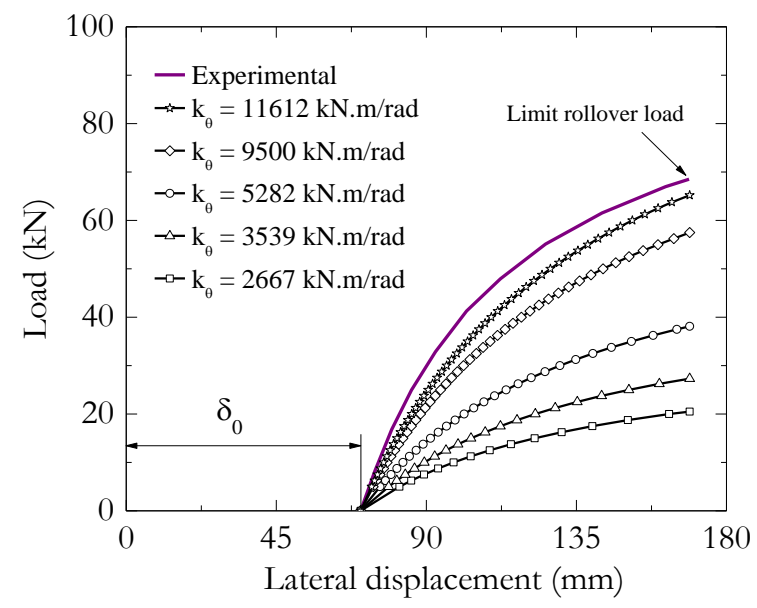

Figure 138 - Load versus lateral displacement considering several values of rotational stiffness

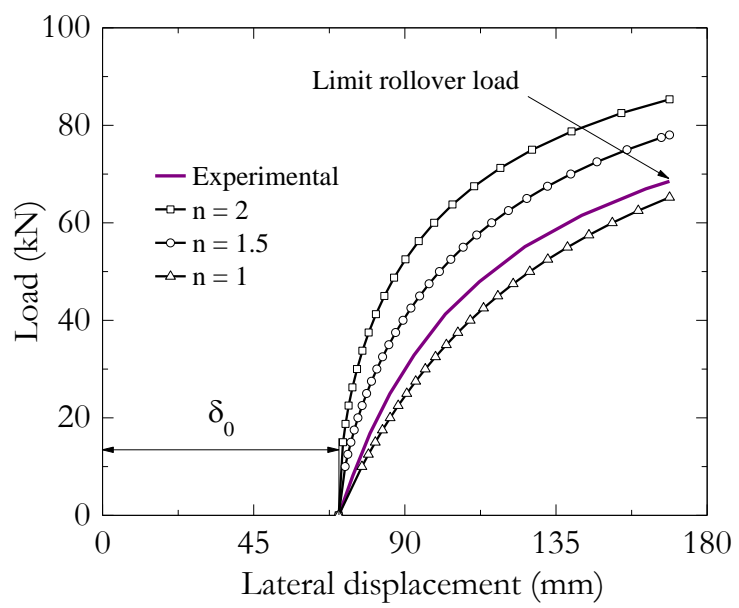

Figure 139 - Load versus lateral displacement considering different values of $n$

From Figure 138, the best approximation for load versus lateral displacement was for the elastic stiffness $k_{\theta}$ of the pad. Besides, the analytical limit load presented high sensitivity to the variation of $k_{\theta}$. Figure 139 shows that the proposed solution better predicted the load versus lateral deflection curve when $n$ is equal to one. For this case, the difference between analytical and experimental limit rollover loads was $13.6 \%$. Furthermore, the increase in $n$ increased the stiffness of the pad/beam system.

\section{Monte Carlo simulation for rollover of precast beams under self-weight}

Monte Carlo simulation is a powerful reliability method to estimate the probability of failure of structures, (CARDOSO et al., 2008). Equation (96) presents the solution for the probability of failure.

$$
p_{f}=P\left[g\left(X_{1}, X_{2}, \ldots, X_{n}\right) \leq 0\right]
$$

Where $X_{1}, X_{2}, \ldots, X_{n}$ are the random variables and $g\left(X_{1}, X_{2}, \ldots, X_{n}\right)$ is the failure function that for the present case is the result of equation (93) combined with the Southwell 
equation minus the self-weight. When $g\left(X_{1}, X_{2}, \ldots, X_{n}\right)$ results negative, that is failure occurs, a Heavy side function is equal to one and otherwise, zero, equation (97).

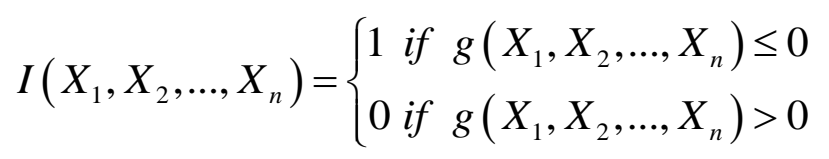

Each failure registered in equation (97) is stored in a sum. Thus, equation (98) enables to determine the probability of failure by the ratio between the sum of the number collapses and the total number of cases studied.

$$
p_{f}=\frac{1}{N} \sum_{i=1}^{N} I\left(X_{1}, X_{2}, \ldots, X_{n}\right)
$$

In the present study, each case was simulated 100000 times, and the probability of failure was determined.

It seems from the experimental results presented by (HURFF; KAHN, 2012b) that a critical parameter for beam stability is the flatness of the bottom surface of the concrete beam in contact with the pads. Without any retrofit of this surface, the load capacity was $71 \%$ smaller for the externally applied load. Then, if a load of $129 \mathrm{kN}$ was the peak load, the correspondent pad stiffness, obtained by equation (92), would be $1022.4 \mathrm{kN} . \mathrm{m} / \mathrm{rad}$. It is worth reminding that the linear rotational pad stiffness for full contact between beam and pad was $24800 \mathrm{kN} . \mathrm{m} / \mathrm{rad}$. Thus, due to the high influence of pad stiffness on stability, an extensive database is required to quantify the surface imperfections statistically and to estimate the probable contact area. However, due to the absence of such database, a possible approach is to consider the rotational stiffness as a random variable considering a possible reduction due to the lack of flatness of the bottom surface of the beam. In the present study, the linear rotational pad stiffness $k_{\theta}$ is established in a range of $5000-150000 \mathrm{kN} . \mathrm{m} / \mathrm{rad}$. For the beginning of the analysis, a value of this range is chosen and considered as the maximum value or the value for which the pad was design. Then, it is admitted a possible initial loss of contact and this stiffness can be reduced randomly up to $25 \%$ or $50 \%$ or 75 $\%$, non-simultaneously. This reduction is called classes $R S$, which are taken as decimal numbers, and are also chosen at the beginning of the analysis. Thus, the pad stiffness utilized in the analysis is a random value between a chosen maximum $k_{\theta}$ and reduced by $R S$. For this purpose, a random number $R N$ is generated between 0 and 1, and the initial random stiffness is calculated by $k_{\theta}(1-R S \times R N)$. If $R N$ is 1 , the pad stiffness reduction is maximum for the 
chosen $R S$, and, if $R N$ is 0 , the pad stiffness is the total value for $k_{\theta}$ with full contact between beam and pad.

Furthermore, the initial lateral displacement and the modulus of elasticity are considered as random variables and represented by a Gaussian distribution. (JCSS, 2001) recommends a coefficient of variation of 0.15 for the modulus of elasticity. For initial imperfections, (COJOCARU, 2012) presented results of 128 field measurements of concrete beams. The measured values exhibited a coefficient of variation of 0.61 . For the present analyses, the mean is arbitrarily adopted for the determination of tolerances. Therefore, one value is adopted for the initial imperfection of $\ell / 1000, \ell / 750, \ell / 500, \ell / 375, \ell / 250$ and $\ell / 125$, which is considered as the mean and the coefficient of variation is the determined by (COJOCARU, 2012). Also, the modulus of elasticity is adopted as $30.8 \mathrm{GPa}$. The rollover criteria were discussed in item 4.

The maximum probability of failure correspondent to a consequence class 3 , as established by (JCSS, 2001), was adopted to compared to the results of Monte Carlo simulations. The recommendation requires that the calculated probability of failure must be smaller or equal to $10^{-4}$. Figure 140 presents a flowchart of the methodology to determine tolerances regarding initial imperfections and required pad stiffness. 


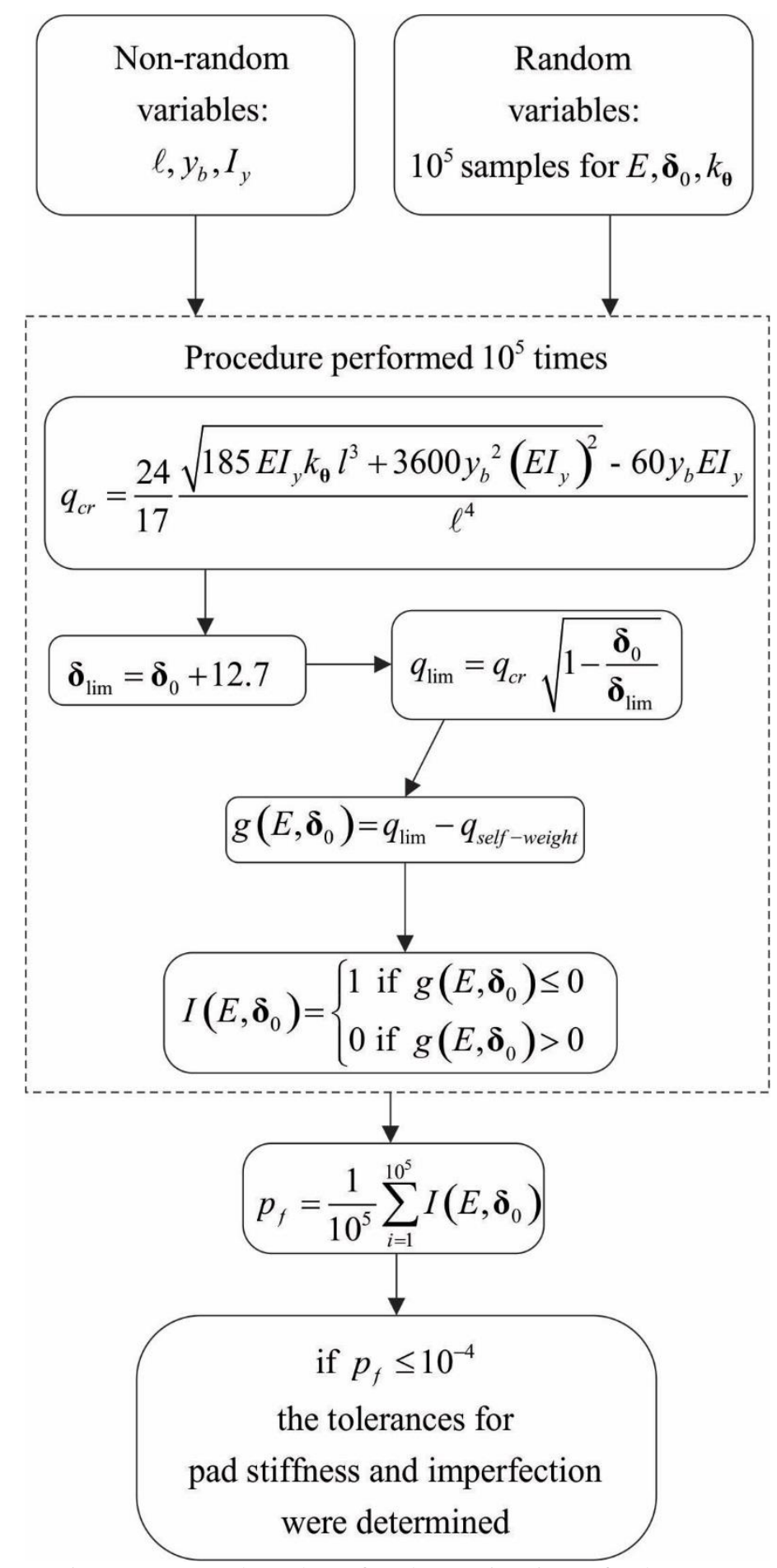

Figure 140 - Flowchart for determination of tolerances

The AASHTO-PCI Bulb-Tee beams were chosen for analyses. The span to depth ratio $\ell / h$ adopted for the beams $\mathrm{BT}-54, \mathrm{BT}-63$, and $\mathrm{BT}-72$, was 22 . Then, the spans were respectively $30.5,35.2 \mathrm{~m}$ and $40.2 \mathrm{~m}$. Table 19 presents the cross-section properties of the beams. 
Table 19 - Geometrical properties of the AASHTO bulb-tee beams

\begin{tabular}{cccc}
\hline Properties & BT-54 & BT-63 & BT-72 \\
\hline Area $\left(\mathrm{mm}^{2}\right)$ & 425642 & 460618 & 495655 \\
$\mathrm{I}_{\mathrm{y}}\left(\mathrm{mm}^{4}\right)$ & $1.554 \times 10^{10}$ & $1.561 \times 10^{10}$ & $1.567 \times 10^{10}$ \\
$\mathrm{yb}_{\mathrm{b}}(\mathrm{mm})$ & 70.26 & 81.65 & 93.08 \\
\hline
\end{tabular}

Figure 141 (a), (b) and (c) presents the results of the simulations of the beam BT-63 accounting for the classes $R S$ equal $25 \%, 50 \%$, and $75 \%$, respectively. The vertical axis corresponds to the probability of failure, and the horizontal axis corresponds to the pad stiffness. For each calculated initial imperfection, the Monte Carlo simulation was performed for an adopted pad stiffness. If the safety criteria was not reached, the pad stiffness was increased, and the Monte Carlo simulations performed again until reaching the established criteria for $p_{f}$. Furthermore, the points alone are cases in which the safety was reached at the first analysis. Then the subsequent results were omitted because the $p_{f}$ was smaller than $10^{-}$ ${ }^{6}$. For the class $R S 75$, it was considered cases of beams with $40 \mathrm{GPa}$ and $50 \mathrm{GPa}$, besides the reference cases.

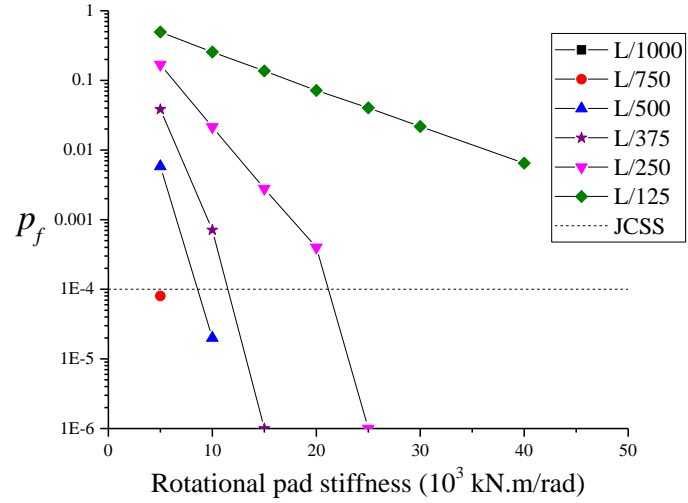

(a)

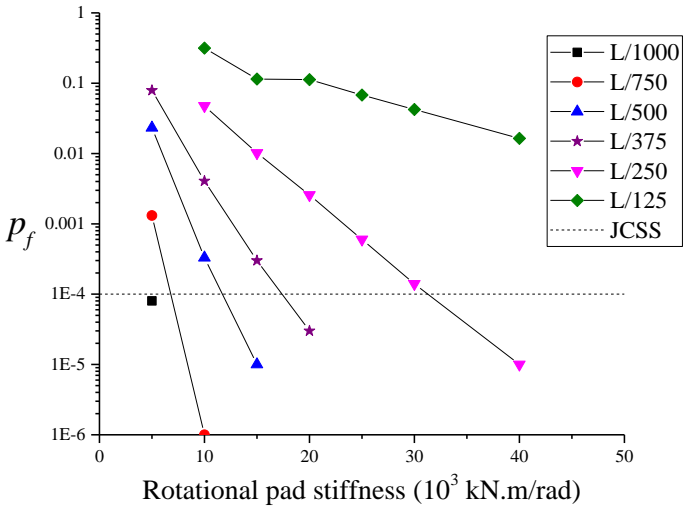

(b)

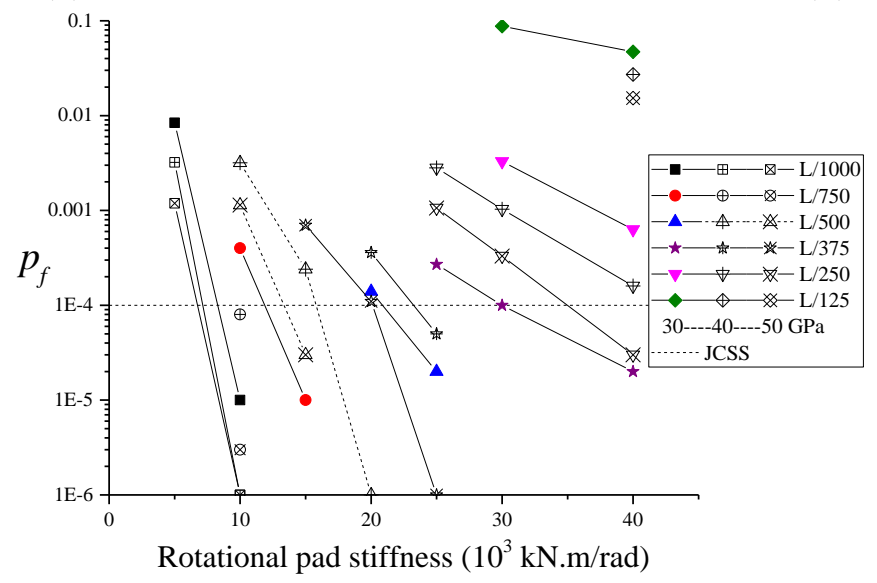

(c)

Figure 141 - Monte Carlo simulation for the beam BT-63 with $R S$ of (a) 25\%, (b) $50 \%$ and (c) 75 $\%$ of the series of AASHTO bulb-tee beams 
The results of Figure 141 can be taken as design charts for beam BT-63, which depends on the assumed hypothesis for construction phases. For example, the designer admits that randomly the pad rotational stiffness can be between the total value and a reduced value by $75 \%$, and assume for a sample set of beams BT-63, the mean initial imperfection of $\ell$ / 500. From Figure 141 (c), if the rotational pad stiffness was $25000 \mathrm{kN} . \mathrm{m} / \mathrm{rad}$, the probability of failure of one beam of the set would be smaller than $10^{-4}$. Figure 142 and Figure 143 presents the design charts for the beams BT-72 and Beam BT-54, respectively. The curves were resumed to present only the values near the limit probability.

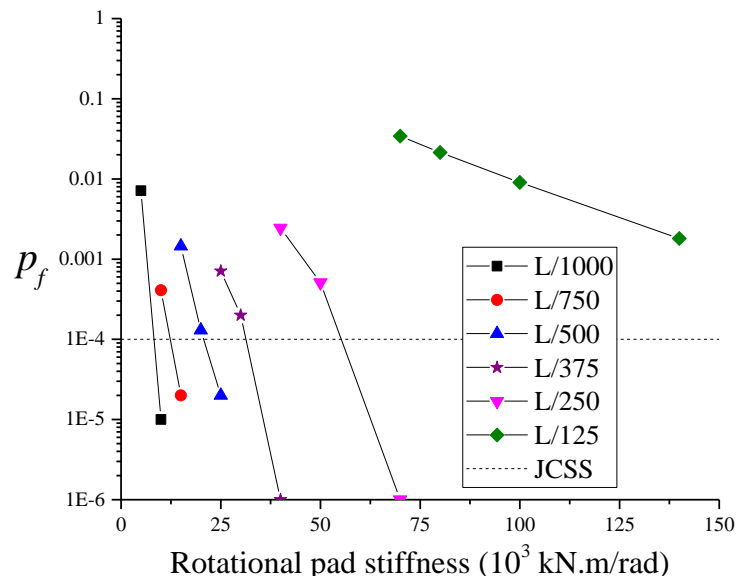

(a)

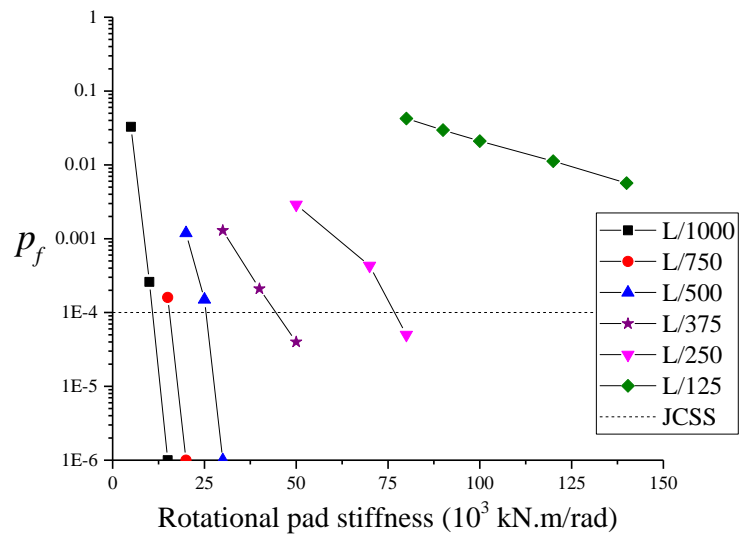

(b)

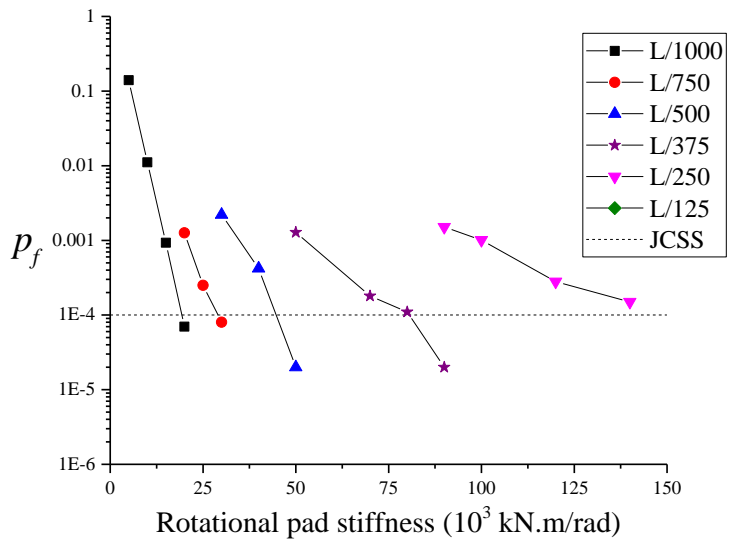

(c)

Figure 142 - Monte Carlo simulation for the beam BT-72 with $R S$ of (a) 25\%, (b) $50 \%$ and (c) 75 $\%$ of the series of AASHTO bulb-tee beams 


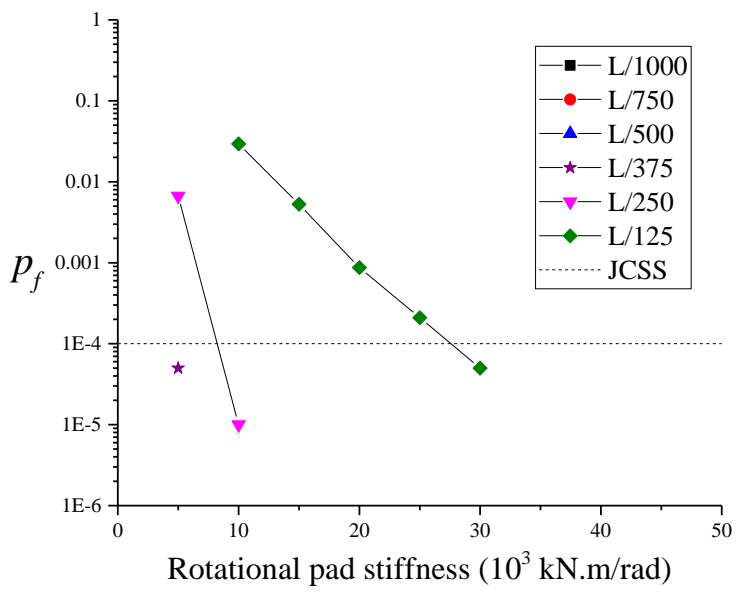

(a)

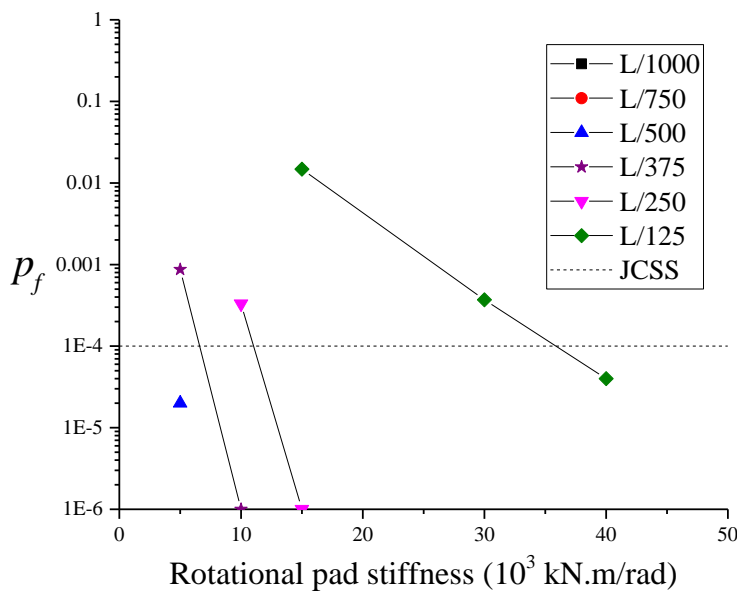

(b)

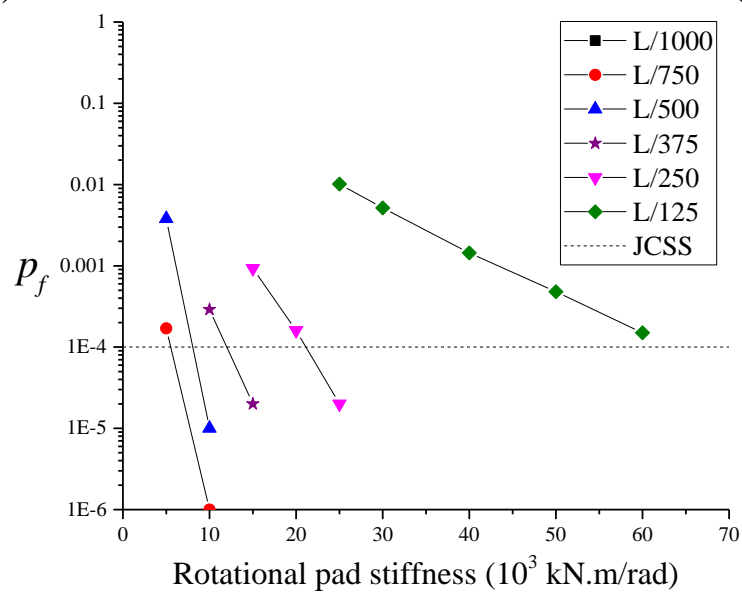

(c)

Figure 143 - Monte Carlo simulation for the beam BT-54 with $R S$ of (a) 25\%, (b) $50 \%$ and (c) 75 $\%$ of the series of AASHTO bulb-tee beams

From Figure 142 (c), taking the beam BT-72 with an assumed mean initial imperfection for a sample set of $\ell / 750$, and the class $R S$ as $75 \%$, the required rotational pad stiffness would be $30000 \mathrm{kN} . \mathrm{m} / \mathrm{rad}$. For this situation, the probability of failure for such one beam of this set would be smaller than $10^{-4}$.

The beam BT-54 tested by (HURFF; KAHN, 2012b) can be taken as an example. The pads utilized had the rotational stiffness of $24800 \mathrm{kN} \cdot \mathrm{m} / \mathrm{rad}$ and the measured initial imperfection was $43.5 \mathrm{~mm}$, which corresponds to $\ell / 700$. This initial imperfection is higher than the limit recommended, for example, by the Spanish code that establishes as the maximum initial imperfection of $\ell / 750$. As reported by (HURFF; KAHN, 2012b), the initial condition of the bottom surface was precarious, but, when supporting the self-weight, the beam did not topple. Figure 143 (c), which represents the class $R S$ of $75 \%$, shows that the beam could have an initial imperfection of $\ell / 250$, with the same designed pad stiffness, that the probability of failure would be smaller than $10^{-4}$. Thus, confirming what was observed 
in practice. Furthermore, if the maximum imperfection was $\ell / 125$, the required pad stiffness would be higher than $60000 \mathrm{kN} \cdot \mathrm{m} / \mathrm{rad}$.

\subsection{NUMERICAL}

\subsubsection{UHPFRC under uniaxial tensile and compressive cyclic loading}

The proposed analytical equations for damage evolutions and stress-strain relationships, which were developed based on the experimental results of the present research, can be incorporated into any constitutive model that couples damage mechanics to the plasticity framework through the effective stress concept. To show the applicability of the present propositions, they are utilized in the Plastic-Damage model implemented in the commercial finite element code ABAQUS to capture the mechanical response of the UHPFRC under tensile, compressive and flexural loads.

One plane element was modeled to simulate and evaluate the proposed damage evolution laws for UHPFRC. Besides, the proposed uniaxial tensile and compressive stressstrain relations were used as hardening/softening evolution laws for the utilized yielding criteria. Figure 144 presents the CPS4R element which is a four-node bilinear plane stress quadrilateral with reduced integration. This approach is performed to evaluate the constitutive model in the unique integration point at the element domain. Displacements were applied in the model, and the unloading/reloading steps were performed with basis on the experimental procedure.

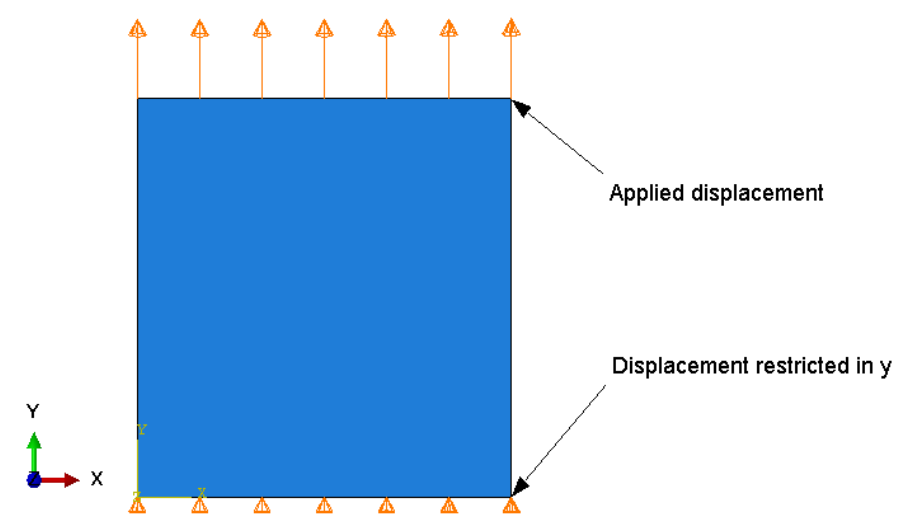

Figure 144 - Finite element model utilized to simulate cyclic tension and compression

Figure 145 shows the comparison of numerical and experimental tensile uniaxial results of UHPFRC under cyclic load. A zoom window of the initial cycles is presented. 

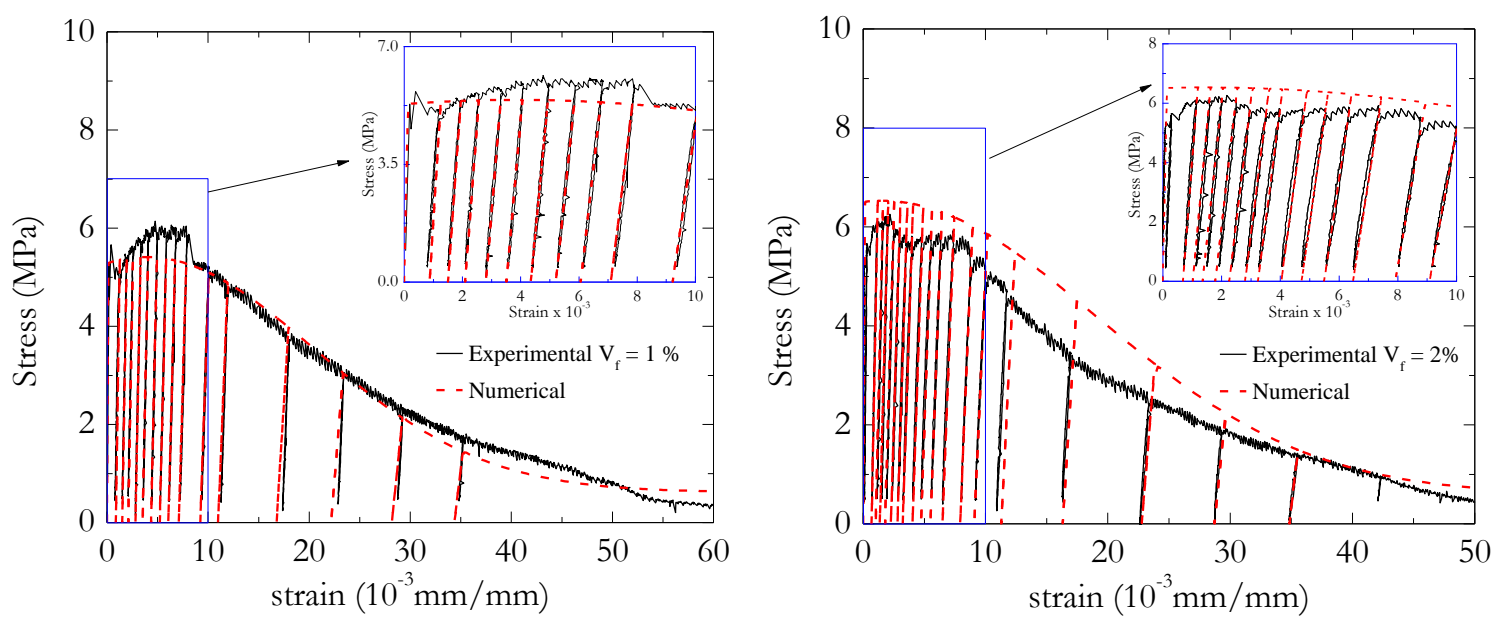

Figure 145 - Comparison between numerical and experimental tensile cyclic results for (a) UHPFRC-1 and (b) UHPFRC -2

The numerical model was capable of predicting the experimental damage evolution, as well as the stress-strain envelopes for both fiber contents of UHPFRC passing by the multiple cracking stage and the softening branch until rupture.

Figure 146 presents the comparison of numerical and experimental uniaxial compressive results for UHPFRC with $1 \%$ and $2 \%$ of fibers.
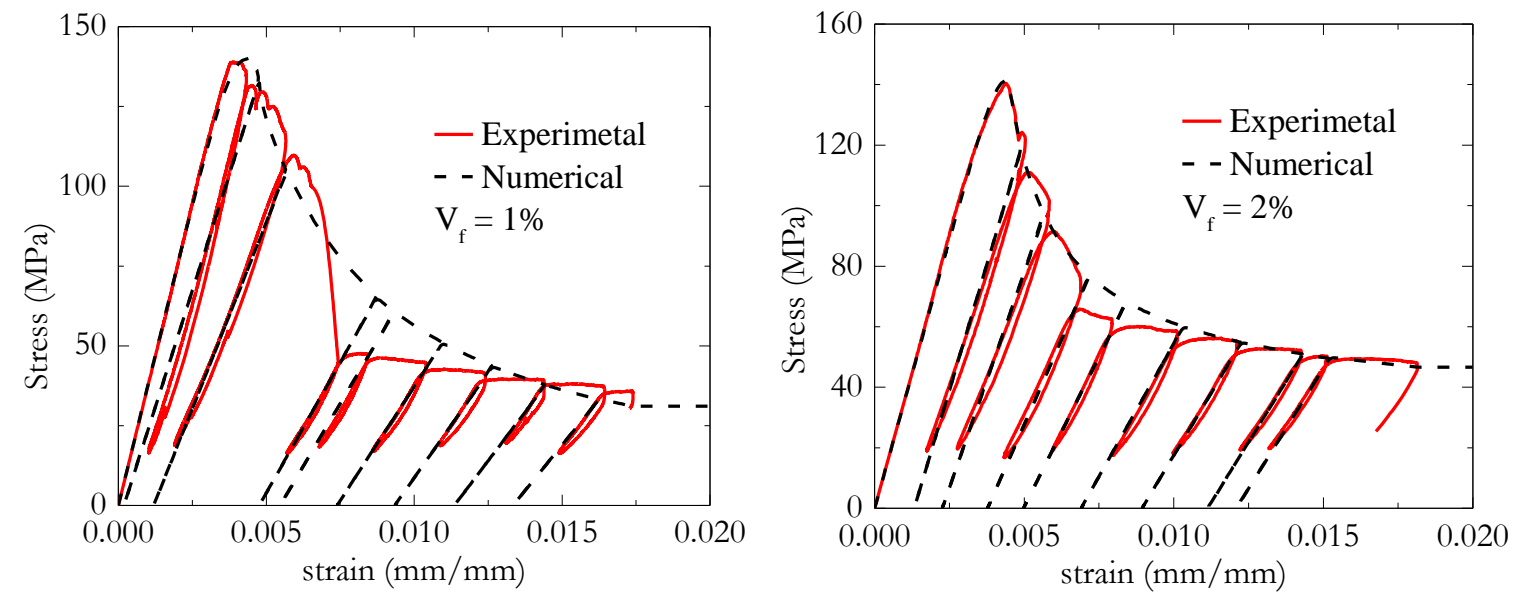

Figure 146 - Numerical response UHPFRC under cyclic load

From Figure 146, the calibrated constitutive plastic-damage model and the proposed evolution laws for compression represented with accuracy the uniaxial compressive behavior in both envelope and degradation evolution.

\subsubsection{UHPFRC under flexural cyclic loading}

Figure 147 shows the numerical model with the finite element discretization and the applied boundary condition utilized to simulate the cyclic four-point bending test. The finite 
element used was the C3D8R which is an eight-node linear brick with reduced integration. Symmetry in the longitudinal z-direction was considered.

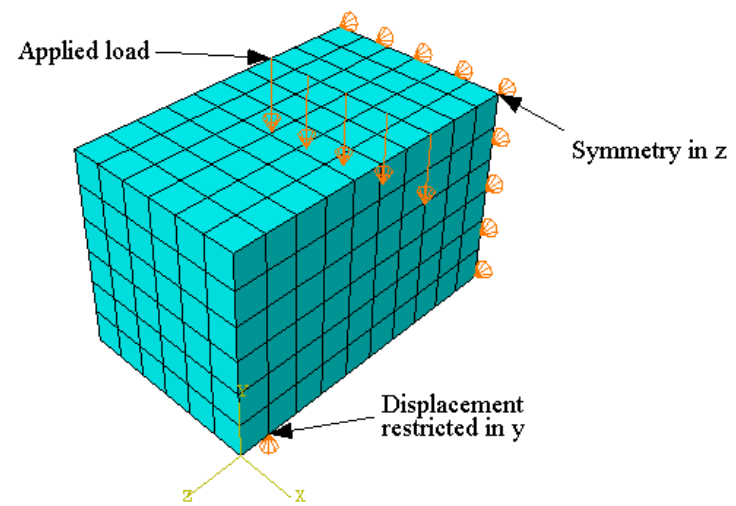

Figure 147 - Finite element discretization of the four-point bending test

Displacements were applied to the model, and Newton-Raphson method was utilized to solve the nonlinear problem. Figure 148 presents the comparison of numerical and experimental cyclic results of the prisms under flexure.
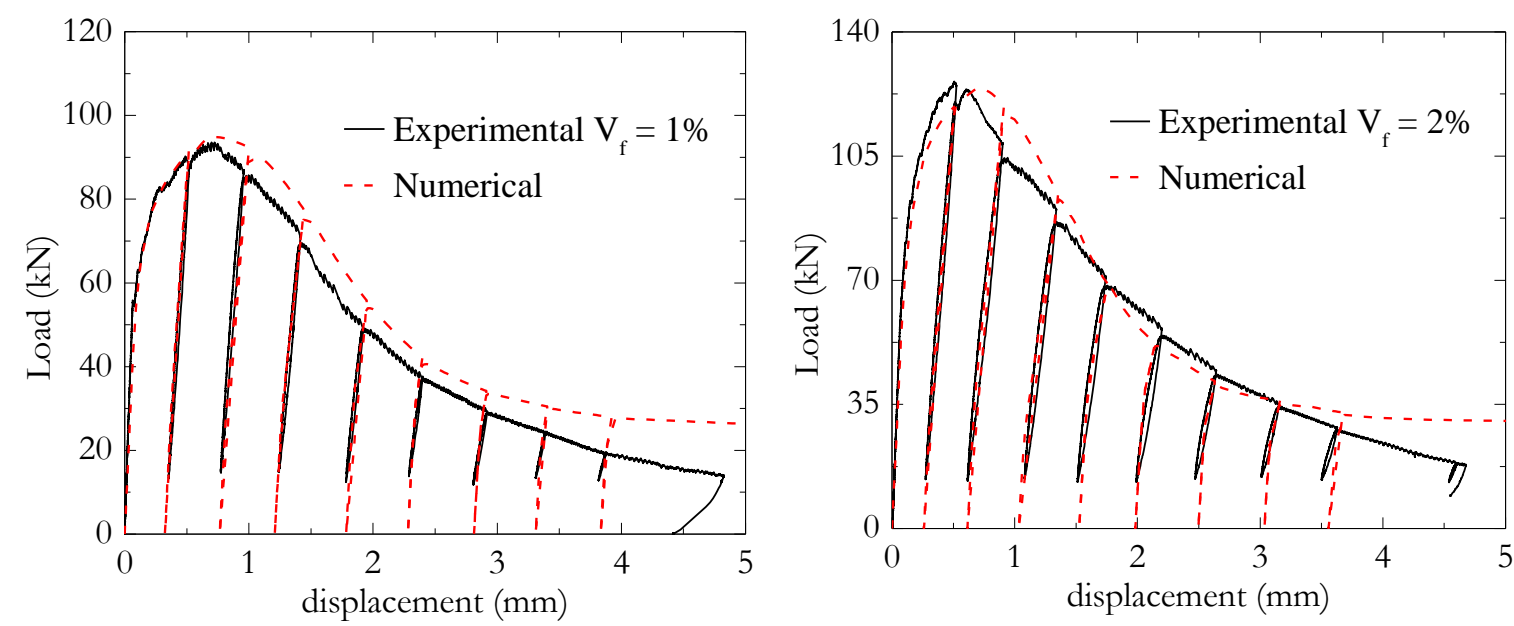

Figure 148- Comparison of cyclic flexural results

The numerical Plastic-Damage model predicted the experimental cyclic curves accurately. Thus, the proposed damage evolution laws and the stress-strain relations for UHPFRC under tension and compression were validated, because the model was capable of predicting the load-bearing capacity of the prisms under flexure. For UHPFRC with $1 \%$ of fibers, the difference between peak loads was $1.32 \%$, while, for UHPFRC with $2 \%$ of fibers, the difference was $1.5 \%$. Besides, the ductility, toughness (the area below the curve) and stiffness degradation were well represented, as can be observed qualitatively in Figure 148.

The quantitative evaluation of the stiffness degradation of UHPFRC prisms under flexure was made through equation (49). Figure 149 presents the comparison of numerical and experimental results of the global damage variable. 


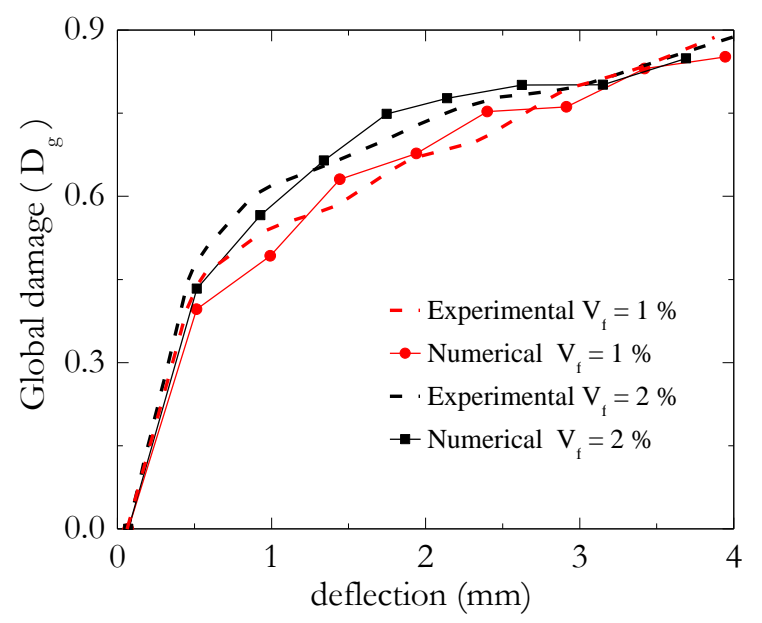

Figure 149 - Comparison of numerical and experimental global damage growth for UHPFRC with $1 \%$ and $2 \%$ of fibers

The numerical model predicted the global damage growth, including the slightly faster increase of damage for prisms with $2 \%$ of fiber content when compared to UHPFRC with $1 \%$ of fibers. Tendency observed experimentally.

Figure 150 presents the numerical and experimental crack pattern for UHPFRC prisms subjected to the four-point bending. Besides, the caption indicates the stage of internal tensile damage variable.

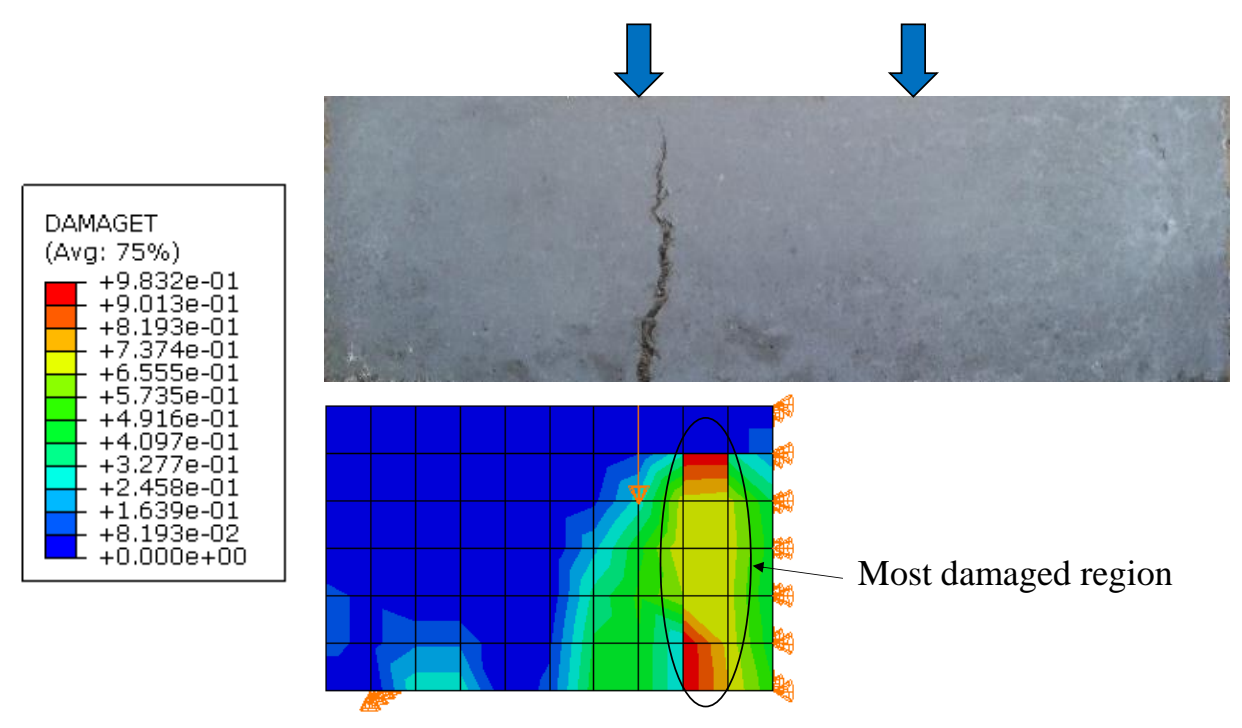

Figure 150 - Crack pattern of the prism with $2 \%$ fiber volume fraction

The higher tensile damage registered in the numerical model was between the midspan and the load at the third middle point. This damaged area almost crossed the whole section height, similarly to what was observed experimentally, Figure 150.

It is worth noting that the hypothesis of isotropic behavior can present some limitation depending on the casting methodology. The limitation is because the casting process with preponderant flow direction can orient the fibers. Furthermore, there are 
researches that present modifications of the Plastic-Damage model available in ABAQUS, (CHI et al., 2017), to account for the influence of discrete fibers on the yielding function and plastic flow direction.

\subsubsection{Numerical modeling of UHPFRC beam lifted by cables}

Two UHPFRC beams were experimentally tested under lifting conditions in the present research. The beam 2 was taken for numerical modeling. The initial lateral displacements and rotations were considered in the model as taken from the total station measurements, as shown in Figure 117. Firstly, this imperfection was manually assigned to the model geometry. Figure 151 presents the top view of beam 2 . The average width of 56.8 $\mathrm{mm}$ was adopted that was the average of the field measurements.

Figure 151 - Top view of the initial configuration of the beam

With such shape, the beam was extruded $510 \mathrm{~mm}$ from this view to set its height and determined the solid beam. The rotation of the section was developed after applying the selfweight in a first step with the essential boundary conditions already employed. The constitutive model utilized for UHPFRC was the calibrated CDP model, presented in item 3.2. Tensile and compressive strengths for UHPFRC were used according to the experimental results and the proposed analytical models. Steel reinforcement was considered with a yield strength of $500 \mathrm{MPa}$ and modulus of elasticity of $210 \mathrm{GPa}$. Figure 152 presents the geometry of modeled reinforcements, which had to follow the beam geometry.

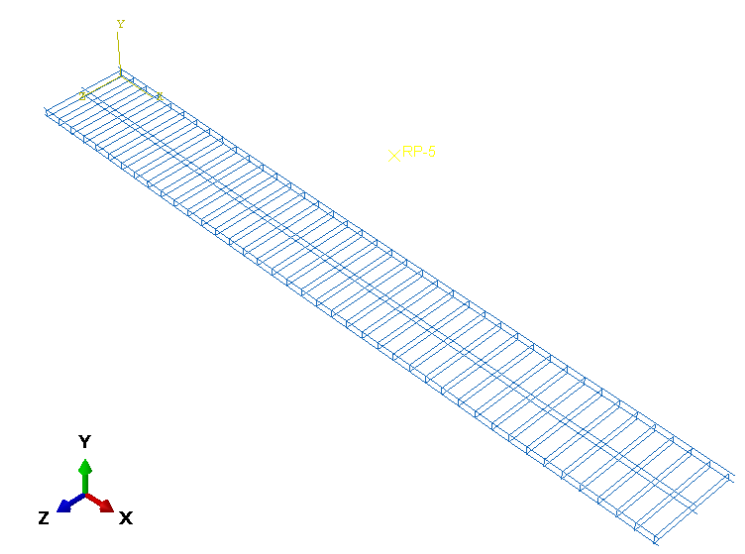

Figure 152 - Detail of the modeled reinforcement 
For reinforcements, 2-node linear 3-D truss finite elements were utilized, which were embedded in the solid elements that represent concrete. For concrete, hexahedral finite elements C3D8R were used, which are 8-node linear brick elements with reduced integration and hourglass control for mesh distortion. Figure 153 (a) and (b) presents the adopted mesh for simulation and the model boundary conditions, respectively.

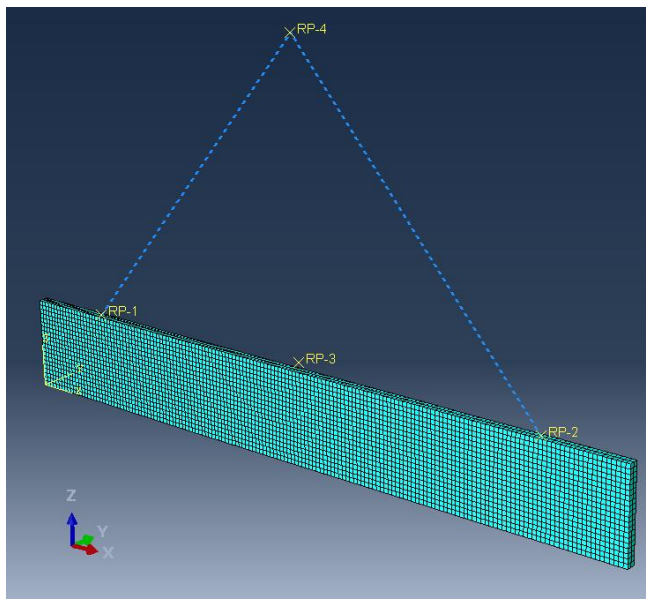

(a)

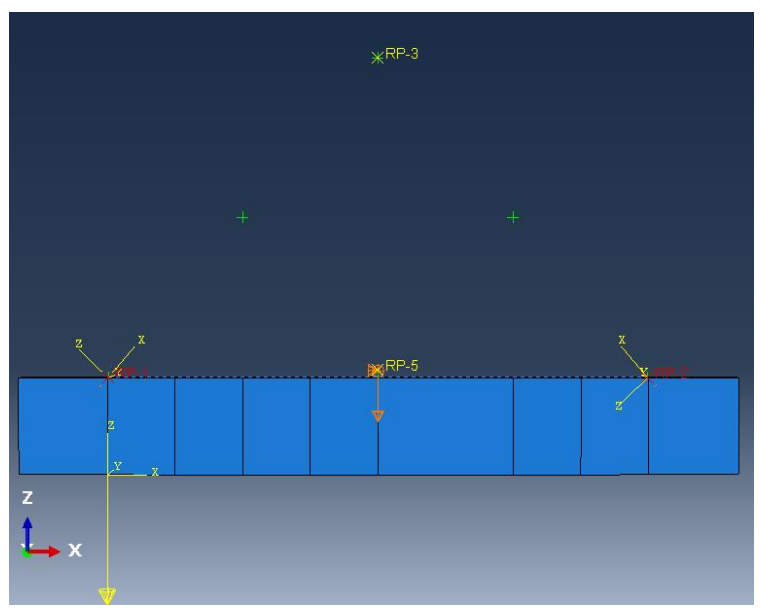

(b)

Figure 153 - Mesh discretization and boundary conditions

It can be seen in the figures that the cables were modeled inclined like in the experiments. Also, the attachment points were positioned with the measured eccentricity relative to the cross-section width of the beam 2. Displacements were constrained in the direction of the cables that are the x-direction of the auxiliary axis, as shown in Figure 153 (b). This direction was inclined 48 degrees relative to the main $\mathrm{x}$-axis of the coordinate system. Furthermore, the displacements were constrained in $\mathrm{z}$ and $\mathrm{x}$ directions at midspan in the point of load, as occurred in the experiments. The first step of analysis was to apply the self-weight incrementally. After, the external load was applied at a point displaced from the top flange to consider the height of the base of the hinge, see Figure 154. From this point, rigid links were fixed to the top flange of the beam at a dimension correspondent to the width of the base of the hinge in the longitudinal direction. 


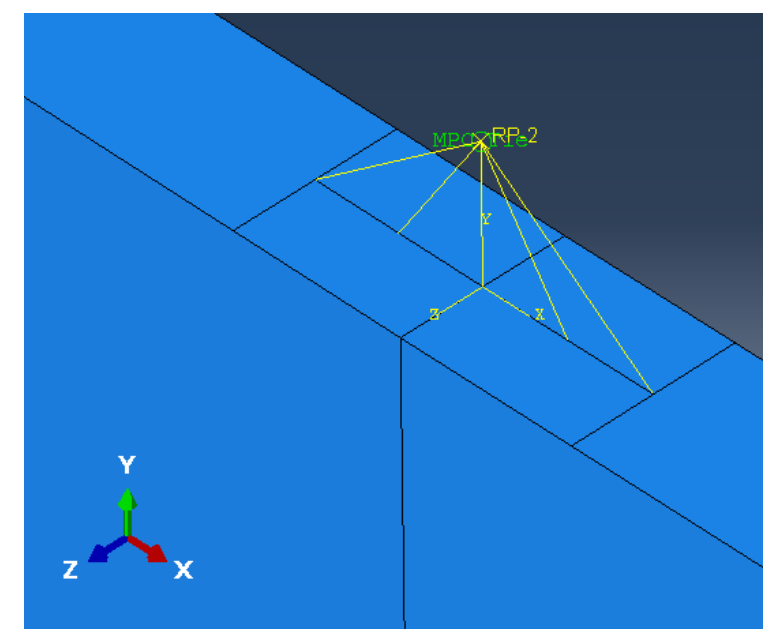

Figure 154 - Constraint to consider the load displaced from the top flange

This model was utilized to perform the geometrical nonlinear analysis (GN) and the geometrical and material nonlinear analysis (GN and MN). As adopted for the model, there is no restriction to the rotation. Figure 155 presents the comparison between GN and combination of GN and MN analysis to the experimental result of the beam 2 .

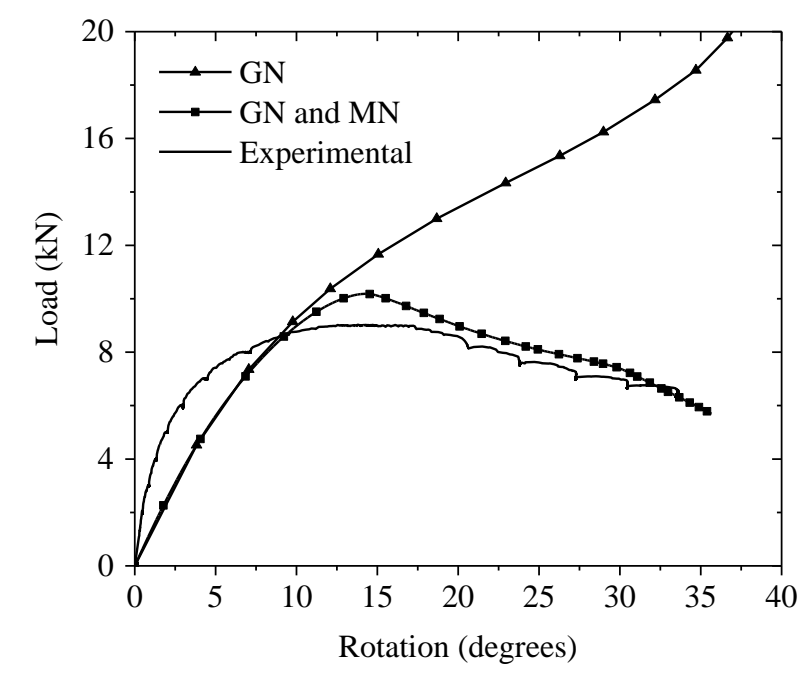

Figure 155 - Numerical and experimental results

The numerical GN and simultaneous GN and MN results were similar until 10 degrees of rotation when the material behavior established the limit load of analysis for combined GN and MN behavior. Also, for this case, the difference in the experimental peak load was $12.8 \%$. It can be seen from the figure that the numerical model presented a difference in the initial stiffness when compared to the experimental test. The initial stiffness can be attributed mainly to the initial modulus of elasticity, the geometry of the beam and boundary conditions. The first two factors were inputted in the model as recorded in tests. Thus, the boundary conditions may have been the major factor in the difference of the initial stiffness. It should be pointed out that the modeled beam was totally free to rotate at the 
supports and the point of load. Then, besides the utilized hinge for rotation at the point of application of load, some mechanism may have increased the initial stiffness of the beam. However, the experimental peak load was not influenced for such mechanism because it was smaller than the numerical results that had no restriction.

\subsubsection{Tilt behavior of concrete beam tested by Lima}

The reinforced concrete beam tested by (LIMA; EL DEBS, 2005) was taken as a reference to simulate the rotational behavior of concrete beams with the Concrete Damage Plasticity (CDP) model of Abaqus. The structural behavior was investigated numerically through three-dimensional Finite Element models using the commercial software (ABAQUS $6.13,2013)$. For concrete, the hexahedral finite element C3D8R was used and for reinforcements, 2-node linear 3-D truss finite element. Figure 156 presents the detail of the reinforcements a the section dimensions of the beam tested by (LIMA; EL DEBS, 2005). The span of this element was $6.29 \mathrm{~m}$.

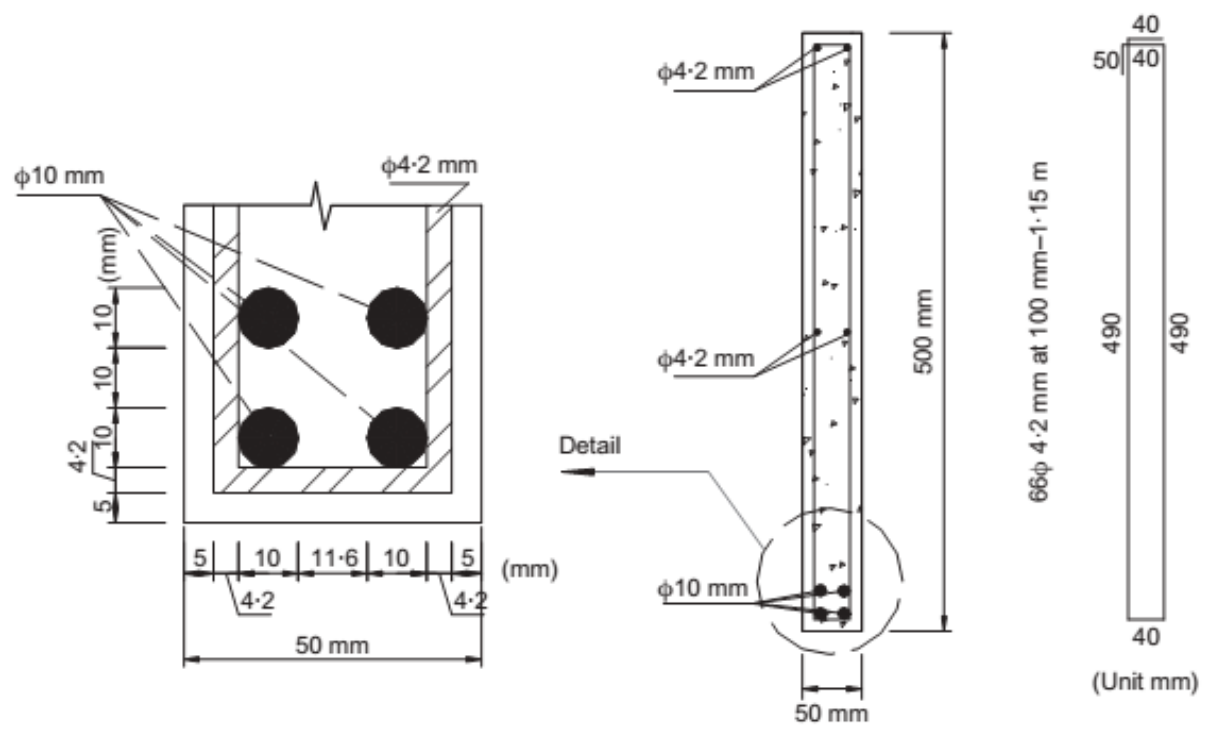

Figure 156 - Reinforcement details of the beam, after (LIMA; EL DEBS, 2005)

The beam V1 of Lima was considered for modeling. The concrete strength was 36.4MPa, and the considered steel yield stress was $500 \mathrm{MPa}$. Figure 157 presents the reinforcements and the mesh discretization for the solid elements. 

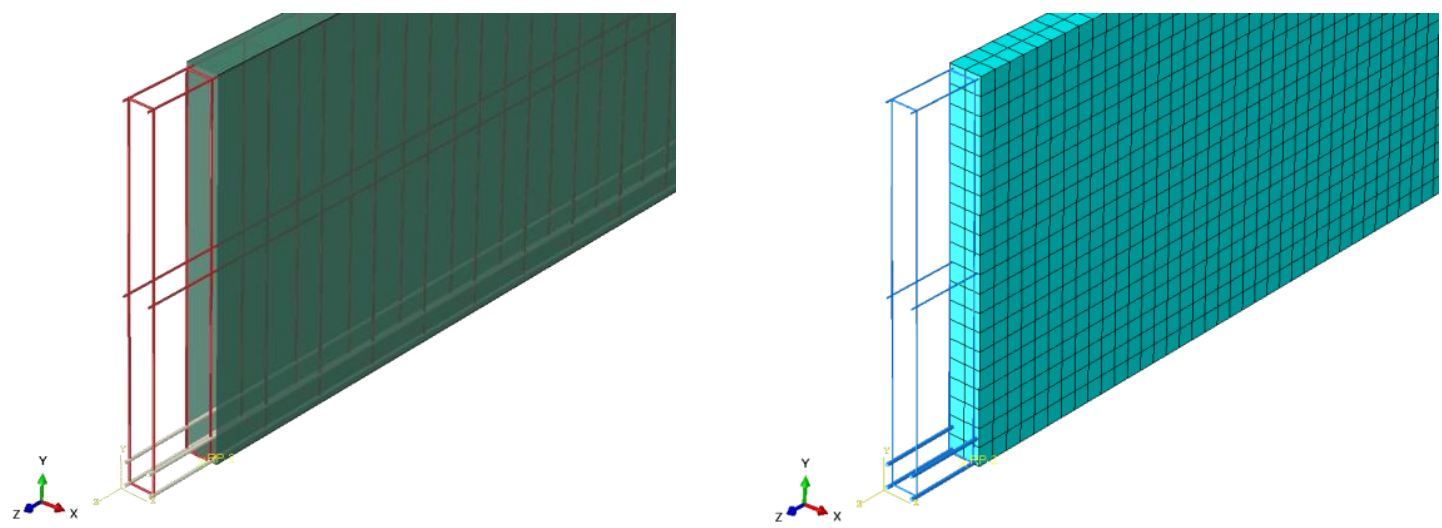

Figure 157 - Mesh discretization and the embedded reinforcements

The plasticity parameters of the CDP model for normal strength concrete were considered as in (LI; HAO; BI, 2017), see Table 20.

Table 20 - Plasticity parameters for CDP model in Abaqus

\begin{tabular}{cc}
\hline$\phi$ & $30^{\circ}$ \\
$m$ & 0.1 \\
$f_{b c 0} / f_{c 0}$ & 1.16 \\
$K_{c}$ & 0.666 \\
$\mu$ & 0.0001 \\
\hline
\end{tabular}

Other inputs in the CDP model are the hardening/softening evolution equations. As well established from plasticity framework, the uniaxial stress-strain responses of a material under tension and compression can represent this evolution. For concrete compression, the proposed equation of (CARREIRA; CHU, 1985) was considered, equation (99).

$$
\begin{aligned}
& f_{c}(\varepsilon)=f_{c m}\left[\frac{\beta \frac{\varepsilon}{\varepsilon_{0}}}{\beta-1+\left(\frac{\varepsilon}{\varepsilon_{0}}\right)^{\beta}}\right] \\
& \beta=\frac{1}{1-\frac{f_{c m}}{\varepsilon_{0} E_{c i}}}
\end{aligned}
$$

Where $f_{c m}, \varepsilon_{0}, E_{c i}$ is the peak stress, peak strain and initial modulus of elasticity, respectively, which are known parameters. The modulus of elasticity was estimated according to the (FIB MODEL CODE, 2010), equation (100).

$$
E_{c i}=E_{c 0} \alpha_{E}\left(\frac{f_{c m}}{10}\right)^{1 / 3}
$$


In which $E_{c 0}$ is $21.510^{3} \mathrm{MPa}, \alpha_{E}$ is 0.9 for limestone aggregates, and $f_{c m}$ is the mean compressive strength.

For tension, a fracture approach was considered in the constitutive model with tensile strength $f_{t}$, and the tensile fracture energy $G_{f}$ determined according to (FIB MODEL CODE, 2010), equations (101) and (102).

$$
\begin{aligned}
& f_{t}=0.3\left(f_{c k}\right)^{2 / 3} \\
& G_{f}=73\left(f_{c m}\right)^{0.18}
\end{aligned}
$$

Where $f_{c k}$ is the characteristic compressive strength. The relation between this quantity and $f_{c m}$ is, according to the model code, $f_{c m}=f_{c k}+8 \mathrm{MPa}$. Furthermore, the steel reinforcement was modeled considered elastic-perfect plastic behavior with yielding stress of $500 \mathrm{MPa}$.

The experimental test was performed by imposing controlled lateral displacements to the beam at the supports. Besides, the beam was vertically supported from below and the twist rotations, at this same points, were possible due to the utilization of a three-dimensional hinge. For more details see the reference (LIMA; EL DEBS, 2005).

The displacements were controlled in the numerical model by the rotation applied at a reference point below the section. All the nodes of the cross-section were attached to this reference point by rigid beam links, which means that when rotation is applied to the reference point, the section rotates. Furthermore, the beam was modeled considering symmetry at midspan to reduce computational cost. Figure 158 presents the comparison between the experimental and numerical results of rotation versus lateral displacement for three points of the section at midspan. Point 7 is at the top, point 8 at middle and point 9 at the bottom of the section. 


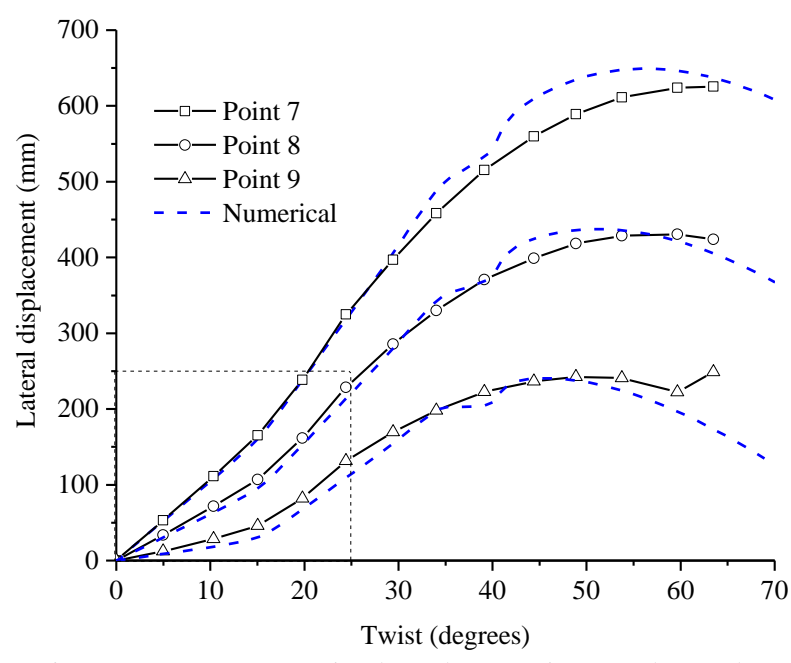

Figure 158 -Numerical and experimental results

The numerical model well predicted the experimental curves. It can be seen from the experimental curves that one significant change in stiffness occurred after the first linear branch and the relation between twist and lateral displacements became nonlinear. The numerical model was capable of predicting these stages. For example, the sharp stiffness reduction near 15 degrees of rotation was predicted. Besides no significant variation of stiffness was observed at the beginning of the test, the cracking rotation was detected numerically at 5.3 degrees. Also, vertical cracks were observed experimentally at one side of the beam showing the preponderance of lateral bending with no torsional cracks. Figure 159 presents the experimental and numerical cracking patterns of the most tensioned side of the beam.
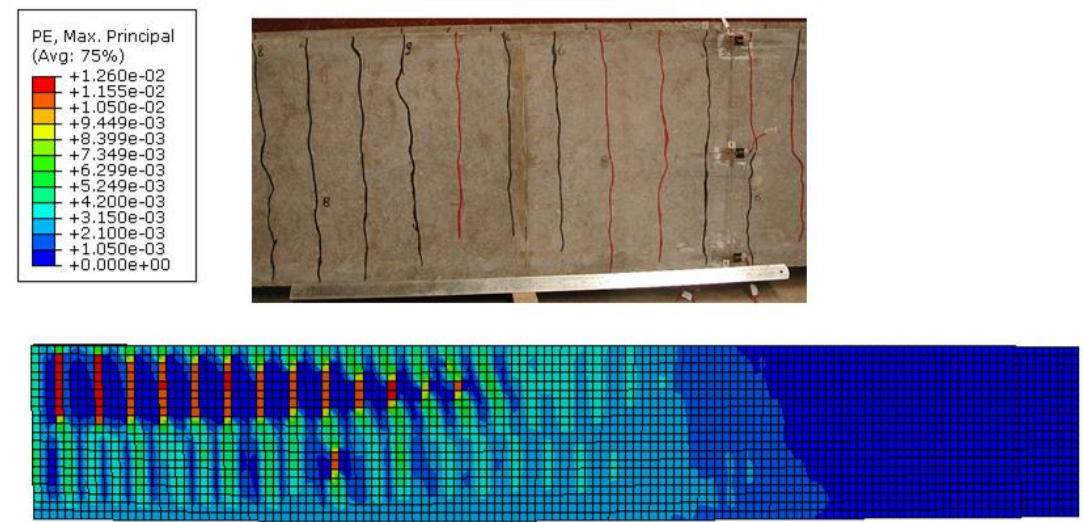

Figure 159 - Experimental and numerical cracking patterns at the tensioned side of the beam

The experimental cracking distribution is possible for NSC structures due to the presence of the steel that promotes tension stiffening. The embedded reinforcements in the numerical model were capable of simulating such phenomenon. 


\subsubsection{Tilt behavior of UHPFRC beam}

The same finite element model developed to predict the response of the beam tested by (LIMA; EL DEBS, 2005) was extended to predict the behavior of UHPFRC beams with $1 \%$ and $2 \%$ of fiber content. Also, imposed rotation at supports, symmetry and the same boundary conditions were considered. The constitutive behavior utilized for UHPFRC was the developed in the present research. Figure 160 presents the comparison between the results of normal strength concrete NSC, and UHPFRC with $1 \%$ and $2 \%$ of fibers, highlighting the cracking rotation of each one with dashed lines. The displacement results correspond to the point at the half height of the beam section.

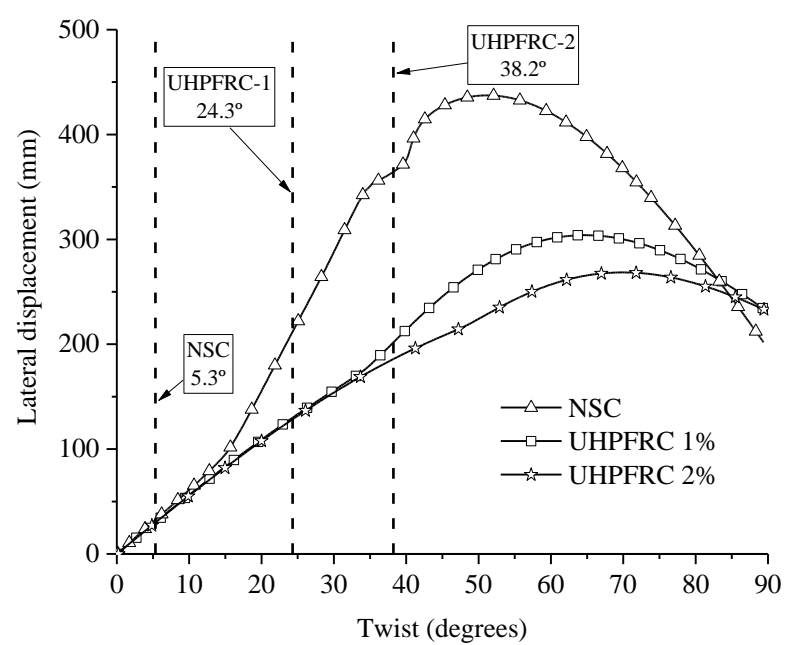

Figure 160 - Comparison of rotation capacity of NSC and UHPFRC beams

It can be seen from the figure that the cracking rotation detected numerically was significantly increased by changing the material. The cracking rotation was 4.6 times higher when considering UHPFRC-1 instead NSC and 7.2 times when utilizing UHPFRC-2 compared to NSC. Also, the fiber content had influence comparing the results for the beams with UHPFRC with $1 \%$ and $2 \%$ of fibers. The increase was 1.6 times for the higher fiber content. The primary cause of this change is the tensile strength of the materials. Also, a decrease in lateral stiffness was observed after first cracking reflected by the increase in the rates of lateral displacements when the beams were further rotated. The rate was higher for NSC beam that can be attributed to the softening behavior of NSC after first cracking. However, the element could carry further lateral displacements due to the tension stiffening caused by the interaction between steel reinforcements and concrete. This behavior enabled the formation of distributed cracking and inhibited the sudden failure of the beam. On the other hand, the rate of lateral displacements for the beams made of UHPFRC was smaller 
because the material presents a strain hardening behavior after first cracking. Thus, when the UHPFRC reaches the matrix cracking strength, there exists the tendency of the distribution of microcracks, as opposed to localization. Then, the lateral stiffness decrease at lower rates, which was more significant for the composite with higher fiber content. It should be pointed out that tension stiffening also occurs. 


\section{CONCLUSIONS}

From the main contributions of this thesis, it is worth mentioning the following conclusions:

- From the material behavior:

An experimental study was carried out to evaluate the cyclic behavior of ultra-high performance fiber reinforced concrete (UHPFRC) under tension, compression and bending with different fiber content.

a) In tension, UHPFRC with $1 \%$ of fibers showed increases of elastic modulus (13.9 $\%)$, cracking stress $(11.4 \%)$, peak stress $(38.6 \%)$, strain at peak stress (2361.5 $\%)$ and toughness $(181.8 \%)$ in comparison to ultra-high performance concrete (UHPC) with no fibers. With $2 \%$ of steel fibers, these increases were, respectively, $28.6 \%, 43.2 \%, 63.6 \%, 976.9 \%$ and $200 \%$ in comparison to UHPC.

b) In compression, UHPFRC with $1 \%$ of fibers showed increases in elastic modulus $(2.8 \%)$, in compression strength $(10.4 \%)$, in axial strain at peak stress $(11.4 \%)$ and toughness $(270.8 \%)$. When $2 \%$ of fibers were used, these increases were $9.6 \%, 14.4 \%, 14.3 \%$ and $362.5 \%$, respectively. Besides this, UHPFRC with 1 $\%$ and $2 \%$ of fibers retained at the end of the test $25.1 \%$ and $33.3 \%$ of the compressive strength, respectively.

c) In bending, UHPFRC (1 \% of fibers) had increased peak load (390\%), displacement at peak load (1768.4\%) and accumulated energy $(41740 \%)$ in comparison to UHPC. These increases were, respectively, $590.5 \%, 1478.9 \%$ and $66940 \%$ in UHPFRC with $2 \%$ of fibers in comparison to UHPC. Fiber bridging and the toughening mechanisms, as fiber/matrix frictional stresses, were responsible for the impressive increase in the post-cracking behavior of UHPFRC in comparison to UHPC.

Damage evolution in tension occurred fast in UHPFRC. Just after first cracks formed, the damage measured was of 0.18 and 0.24 for UHPFRC with $1 \%$ and $2 \%$ of fibers, respectively. In this phase, the higher the fiber content, the higher was damage. The higher volume occupied by interfacial transition zone probably made UHPFRC with $2 \%$ of fibers degrade faster in this phase, as the role of fibers at the beginning was still small. However, during multiple cracking formation, fiber bridging is activated as start to occur phenomena 
like debonding and frictional slip. Then, the fibers contribute more to the composite response. Damage at the end of this phase was, respectively, 0.8 and 0.85 for UHPFRC with $1 \%$ and $2 \%$ of fibers. During the third phase of behavior (localization phase) the higher fiber content reduced the rate of damage evolution. At the end of the test, the damage was of 0.91 and 0.93 , respectively, with $1 \%$ and $2 \%$ of fibers. It is possible to notice that after fiber bridging was activated, the higher volumetric ratio of fiber retained slight better damage evolution. In compression, damage only appeared beyond an axial strain of 0.004 and increased more gradually in comparison to the behavior in tension. In this case, the damage was higher during the entire test for UHPFRC with lower fiber content. Damage at the end of the test was of 0.61 and 0.55 for UHPFRC with $1 \%$ and $2 \%$ of fiber content, respectively. The damage evolution during the bending test was similar to what happened in tension, which occurred fast at the beginning of the test and from that grew faster for the higher volumetric content of fiber. After crack bridging was activated, damage evolution was slower for UHPFRC with $2 \%$ of fibers. For high strain values, at the end of the test, the damage was the same for both mixtures (0.84).

Equations were proposed to describe damage evolution and stress-strain curves of UHPFRC for both tension and compression. These equations were used in finite element simulations through a calibrated constitutive model coupling damage and plasticity in Abaqus. The numerical model was capable of predicting damage evolution and cyclic envelopes during all the phases of the tension, compression and bending tests. Thus, the proposed equations can be applied to any constitutive model based on damage mechanics coupled to the framework of plasticity. Besides, the proposed equations can be of great importance for future research dealing with damage detection in structures made of UHPFRC.

The obtained results represent properties of UHPFRC produced with steel fibers with $13 \mathrm{~mm}$ of length and $0.2 \mathrm{~mm}$ in diameter. UHPFRC produced with other types of fibers may have different behavior. Besides, in the present research fibers were considered in a random distribution and alignment. No efforts were made to align fiber to a preferential direction. This subject may be of interest in future research.

- From the proposed formulation for beams supported by bearing pads:

The present study focused on developing analytical solutions to predict rollover loads of precast beams supported by bearing pads. The equations included situations of beams subjected to concentrated midpoint load and symmetrical two point loads to predict the 
experimental results available in the literature, which have these load configurations. Besides, this research presented another solution to predict the critical self-weight, which is characteristic of the construction phase.

The proposed solutions predicted accurately the load versus lateral displacement behavior of the experimental results of prestressed concrete beams presented in the literature. As discussed, prestress has no effect on bifurcation problem for beams centrally prestressed relative to lateral flexural deflections. Furthermore, the analytical limit rollover loads presented good approximation compared to the experimental data. The analytical solution differed $4.37 \%$ to the experiment of Hurff and Kahn [5] and $13.6 \%$ for the beam tested by Consolazio and Hamilton [6]. Also, the exponent $n$ of the equation of Southwell that better adjusted to the experimental results was 1 . Furthermore, the available equations published in the literature were inapplicable to the problems, mainly due to not consider the effect of loading position and the type of loading.

For the construction situation, when only self-weight acts, the AASHTO-PCI BulbTee beams were investigated concerning initial imperfection tolerances and required rotational pad stiffness by Monte Carlo simulations. The results showed the high influence of initial imperfection and reduced rotational stiffness of the pads on beam stability and enabled to estimate the beam safety during this construction phase by the presented charts. Naturally, the effect of slenderness was also observed. Thus, the results are recommended for the design and verification of such beams with similar span to depth ratio of 22 .

Also, the proposed solutions with different loading conditions are a valuable tool for further development of experimental research given the few existing ones. Besides, the authors highlight the importance of more investigation about the lack of flatness of the beams bottom flanges due to the significant influence on beam stability.

- From the structural behavior of UHPFRC beams lifted by cables:

The load capacity of the UHPFRC beams was substantially reduced due to the lifting support conditions. The lifting cables do not restrict the beam for lateral displacements and rotations. Thus, the UHPFRC beam buckles long before reaching the flexural load capacity. If the beam was restricted at supports, the load would $35 \mathrm{kN}$, according to a flexural model. However, the average load capacity was $9.4 \mathrm{kN}$, which is 3.7 times smaller.

The UHPFRC beams started to crack close the peak load, with a rotation capacity until the peak of 12.5 and 14.2 degrees for beam 1 and beam 2, respectively. Thus, linear material behavior governed the pre-peak range combined with large displacements. This 
hypothesis was considered in the analytical propositions, based on the experimental evidence.

The initial configuration of the beams during the test was known due to the use of the total station in the measurements. The methodology is simple to implement with great precisions, inclusive higher than the string potentiometers. This procedure is highly recommended for measurements in the field that enables the constructors to know a priori the magnitude of initial imperfections during the erection stages. The shape of the two tested UHPFRC beam was detected to be different, which also governed the load rotation curves.

Given the no existence of an analytical solution to predict the load capacity of the present tests, analytical closed-form solution was proposed. The hypotheses for the equation were based on experimental evidence, as rigid body rotation and lateral flexural behavior. The equation predicted with good approximation the experimental instability loads. Furthermore, an analytical comparison was made for lateral instability capacity of beams made of ordinary concrete and UHPFRC. The UHPFRC beam presented a load capacity 32 $\%$ higher. Thus, besides the concern with instability problems, the use of UHPFRC presents a favorable tendency for the design of more slender elements for structural application.

- From the numerical studies performed with normal strength and UHPFRC beams:

The developed numerical models could predict the experimental behavior of the UHPFRC beam tested in the present work and the experimental study developed by (LIMA; EL DEBS, 2005). In the first numerical study, the reduced load bearing capacity due to the lifting condition was predicted with a difference of $12.8 \%$. The main difference between numerical and experimental results was the initial stiffness of the load versus rotation curve. The numerical model was free for rotation. Then, a hypothesis for such difference is that it occurred any mechanism at the designed hinge that increased the initial stiffness of the test. Besides, the beam tested by (LIMA; EL DEBS, 2005) was simulated with great accuracy including predicting the tension stiffening effect. Also, the model was extended to simulate the same beam with UHPFRC with $1 \%$ and $2 \%$ of fibers as material. The numerical results showed that the beams with this material have a higher capacity of rotation before cracking, mainly due to the higher tensile strength and also due to the strain hardening behavior. Besides, the higher modulus of elasticity decreases the rate of increase of lateral displacements significantly. 


\section{PROSPECTS FOR FUTURE RESEARCH}

Research on UHPFRC is an open field that requires more investigations to the development of code recommendations and the material to be more utilized in the construction industry. The efforts of researchers have proved the potential of this cementbased composite. From the research developed by the author of the present thesis, there are some recommendations for future research in the scope of material and thin elements made of UHPFRC.

Material:

- Development of damage evolution models for tension and compression based on constants with physical meaning;

- An analytical model to predict the uniaxial cyclic behavior including the experimentally observed hysteretic behavior. The investigation on the mechanisms involved in this last topic is also an open filed for research;

- Quantification of each damage mechanism occurred during severe loading and at different strain rates.

Thin structural members:

- Tests of beams lifted by cables are rare. Then, such tests are relevant for beams made with any cement based composite. Also, the development of experimental research with prestressed beams are highly recommended;

- Analytical and numerical parametrical studies with the proposed formulations and developed numerical models can be of high importance to determine limits and tolerances for concrete beams under transitory phases;

- The utilization in the field of the methodology to measure initial displacements with total station can be beneficial to obtain a database and propose recommendations. 


\section{REFERENCES}

EHE-08. Comisión Permanente del Hormigón. Instrucción de Hormigón Estructural. Ministerio de Fomento. 3th Edition, 2009.

JSCE. Standard Specifications for Concrete Structures. Structural Performance Verification. 2002.

AFGC. Ultra-high performance fiber-reinforced concrete. Recommendations. Paris. Revised edition, 2013.

ASTM C1611 / C1611M-14, Standard Test Method for Slump Flow of SelfConsolidating Concrete, ASTM International, West Conshohocken, PA, 2014, www.astm.org.

ASTM C1609 / C1609M-12, Standard Test Method for Flexural Performance of Fiber-Reinforced Concrete (Using Beam With Third-Point Loading), ASTM International, West Conshohocken, PA, 2012, www.astm.org. (n.d.), ASTM Inter.

ASTM C469 / C469M-14, Standard Test Method for Static Modulus of Elasticity and Poisson's Ratio of Concrete in Compression, ASTM International, West Conshohocken, PA, 2014, www.astm.org. (n.d.).

ABAQUS 6.13. Abaqus analysis user's manual ProvidenceDassault Systèmes, Version 6.13-1, , 2013.

ABBAS, S.; NEHDI, M. L.; SALEEM, M. A. Ultra-High Performance Concrete: Mechanical Performance, Durability, Sustainability and Implementation Challenges. International Journal of Concrete Structures and Materials, [s. 1.], v. 10, n. 3, p. 271$295,2016$.

AGHDASI, Parham et al. Mechanical properties of a highly flowable ultrahighperformance fiber-reinforced concrete mixture considering large-size effects. In: HPFRCC7, 7TH RILEM WORKSHOP ON HIGH PERFORMANCE FIBER REINFORCED CEMENT COMPOSITES 2015, Anais... [s.l: s.n.]

AL-OSTA, Mohammed A. Exploitation of Ultrahigh-Performance Fibre-Reinforced 
Concrete for the Strengthening of Concrete Structural Members. Advances in Civil Engineering, [s. 1.], p. 1-12, 2018.

ANDERSON, A. R. Lateral stability of long prestressed concrete beams. PCI Journal, [s. 1.], v. 16, n. June, p. 7-9, 1971.

BAIRÁN, J. M.; CLADERA, A. Collapse of a precast concrete beam for a light roof. Importance of elastomeric bearing pads in the element's stability. Engineering Failure Analysis, [s. 1.], v. 39, p. 188-199, 2014.

BAŽANT, Z. P.; CEDOLIN, Luigi. Stability of Structures: Elastic, Inelastic, Fracture, and Damage Theories. [s.1.] : Courier Corporation, 1991.

BENTUR, Arnon; MINDESS, Sidney. Fibre Reinforced Cementitious Composites. ed. 2 ed. London, UK; New York, US: Taylor \& Francis, 2007.

BENZERZOUR, Mahfoud et al. A bibliography on the analytical model of the mechanical behaviour in uniaxial tension of fibre concrete: Application to concrete reinforced with fibres and powders from recycling of thermoset composite materials. Construction and Building Materials, [s. 1.], v. 131, p. 214-228, 2017. Disponível em: <http://dx.doi.org/10.1016/j.conbuildmat.2016.11.060>

BURGOYNE, C. J.; STRATFORD, T. J. Lateral instability of long-span prestressed concrete beams on flexible bearings. The Structural Engineer, [s. 1.], v. 79, p. 23-26, 2001.

BUTTIGNOL, T. E. T.; SOUSA, J. L. A. O.; BITTENCOURT, T. N. Ultra HighPerformance Fiber-Reinforced Concrete (UHPFRC): a review of material properties and design procedures. Revista IBRACON de Estruturas e Materiais, [s. 1.], v. 10, n. 4, p. 957-971, 2017. Disponível em: $<$ http://www.scielo.br/scielo.php?script=sci_arttext\&pid=S1983$41952017000400957 \& \operatorname{lng}=$ en \&tlng=en>

CANDAPPA, D. C.; SANJAYAN, J. G.; SETUNGE, S. Complete triaxial stressstrain curves of high-strength concrete. Journal of Materials in Civil Engineering, [s. 1.], v. 13, n. June, p. 209-215, 2001.

CARDOSO, João B. et al. Structural reliability analysis using Monte Carlo 
simulation and neural networks. Advances in Engineering Software, [s. 1.], v. 39, p. 505$513,2008$.

CARDOSO, M. T. S. A.; LIMA, M. C. V. Physical and geometrical non-linear behavior of precast beams on elastomeric supports. Ibracon structures and materials journal, [s. 1.], v. 11, n. 7, p. 183-202, 2018.

CARREIRA, Domingo J.; CHU, Kuang-han. Stress-strain relationship for plain concrete in compression. ACI Journal Proceedings, [s. 1.], v. 82, n. 6, p. 797-804, 1985. Disponível em: <http://www.concrete.org/Publications/ACIMaterialsJournal/ACIJournalSearch.aspx?m=d etails \&ID=10390>

CHAJES, A. Principles of structural stability theory. [s.1.] : Prentice Hall, 1974.

CHEN, Tiefeng; GAO, Xiaojian; REN, Miao. Effects of autoclave curing and fly ash on mechanical properties of ultra-high performance concrete. Construction and Building Materials, [s. 1.], v. 158, p. 864-872, 2018.

CHENG, Jung-June R.; YURA, Joseph A. Lateral Buckling Tests on Coped Steel Beams. Journal of Structural Engineering, [s. 1.], v. 114, n. 1, p. 16-30, 1988.

CHI, Yin et al. Finite element modeling of steel- polypropylene hybrid fiber reinforced concrete using modified concrete damaged plasticity. Engineering Structures, [s. 1.], v. 148, n. June, p. 23-35, 2017. Disponível em: <http://dx.doi.org/10.1016/j.engstruct.2017.06.039>

COJOCARU, Razvan. Lifting Analysis of Precast Prestressed Concrete Beams. 2012. Virginia Polytechnic Institute, [s. 1.], 2012.

CONSOLAZIO, Gary R.; HAMILTON, H. R. Experimental Validation Of Bracing Recommendations For Long-Span Concrete Girders. Florida.

DE LA FUENTE ANTEQUERA, Albert; CAVALARO, Sergio Henrique Pialarissi; GARCÍA, Jesús Miguel Bairán. Análisis de un caso de inestabilidad lateral de un viga de hormigón pretensado de gran luz en fase de izado. Hormigón y Acero, [s. 1.], 2017. Disponível em: <http://linkinghub.elsevier.com/retrieve/pii/S0439568917300244> 
DESAYI, Prakash; KRISHNAN, S. Equation for stress-strain curves of concrete. ACI Journal Proceedings, [s. 1.], v. 61, n. 3, p. 345-350, 1964.

DIAMOND, Sidney; SAHU, Sadananda; THAULOW, Niels. Reaction products of densified silica fume agglomerates in concrete. Cement and Concrete Research, [s. 1.], v. 34, n. 9, p. 1625-1632, 2004.

EL-HELOU, Rafic G. et al. Ultra-High Performance Concrete Compression and Fracture Response Parameters for Lattice Discrete Particle Model Simulations. In: FIRST INTERNATIONAL INTERACTIVE SYMPOSIUM ON UHPC 2016, Anais... [s.l: s.n.] Disponível em: <https://www.extension.iastate.edu/registration/events/UHPCPapers/UHPC_ID15.pdf>

EL DEBS, Mounir Khalil. Concreto pré-moldado: fundamentos e aplicações. second edi ed. [s.l: s.n.].

ESCOBAR-CASTILLO, Martha Nerida et al. Experimentelle und numerische Untersuchungen zum Beulen von dünnen Scheiben aus UHPFRC unter Druckbeanspruchung. Beton- und Stahlbetonbau, [s. 1.], v. 107, n. 1, p. 2-14, 2012.

FANELLA, David A.; NAAMAN, Antoine E. Stress-Strain Properties of Fiber Reinforced Mortar in Compression. ACI Journal Proceedings, [s. 1.], v. 82, n. 4, p. 475483, 1985.

FARNAM, Y. et al. Behaviour of Slurry Infiltrated Fibre Concrete ( SIFCON ) under triaxial compression. Cement and Concrete Research, [s. 1.], v. 40, n. 11, p. 1571-1581, 2010. Disponível em: 〈http://dx.doi.org/10.1016/j.cemconres.2010.06.009>

FEHLING, Ekkehard et al. Ultra-High Performance Concrete UHPC. Berlim, Germany: Wilhelm Ernst \& Sohn, 2014.

FIB MODEL CODE. Fédération Internationale. fib model code for concrete structuresBerlin, Germany, 2010.

FLANDERS, Lauren; RUSHING, Todd; LANDIS, Eric. Energy Dissipation Mechanisms in the Fracture of Fiber Reinforced Ultra High Performance Concrete. In: INTERNATIONAL SYMPOSIUM ON ULTRA HIGH PERFORMANCE CONCRETE 
2016, Kassel, Germany. Anais... Kassel, Germany

GHAFARI, Ehsan; COSTA, Hugo; JÚLIO, Eduardo. Critical review on eco-efficient ultra high performance concrete enhanced with nano-materials. Construction and Building Materials, [s. 1.], v. 101, p. 201-208, 2015. Disponível em: <http://dx.doi.org/10.1016/j.conbuildmat.2015.10.066>

GRASSL, Peter; JIRÁSEK, Milan. Damage-plastic model for concrete failure. International Journal of Solids and Structures, [s. 1.], v. 43, n. 22-23, p. 7166-7196, 2006.

GRAYBEAL, Benjamin A. Compressive Behavior of Ultra-High-Performance Fiber-Reinforced Concrete. [s. 1.], v. 146, n. 104, 2007.

GU, Chun Ping; YE, Guang; SUN, Wei. Ultrahigh performance concrete-properties, applications and perspectives. Science China Technological Sciences, [s. 1.], v. 58, n. 4, p. 587-599, 2015.

GU, Chunping et al. Investigation of Microstructural Damage in UltrahighPerformance Concrete under Freezing-Thawing Action. Advances in Materials Science and Engineering, $[\mathrm{s} . \quad 1],$.$\quad v. 2018, p. 1-9, 2018. Disponível em:$ <https://www.hindawi.com/journals/amse/2018/3701682/>

HANSELL, W.; WINTER, G. Lateral stability of reinforced concrete beams. Journal of the American Concrete Institute, [s. 1.], v. 31, n. 3, p. 193-213, 1959.

HANSSEN, Trygve. EMMA Elkem Material Mix AnalyzerElkem AS 2012, , 2012.

HARISH, Kizhakkumodom Venkatanarayanan; DATTATREYA, Jamboor Krishnamurthy; NEELAMEGAM, Meyappan. Experimental investigation and analytical modeling of the $\mathrm{r}$ - e characteristics in compression of heat-treated ultra-high strength mortars produced from conventional materials. Construction and Building Materials, [s. 1.], v. 49, p. 781-796, 2013. Disponível em: <http://dx.doi.org/10.1016/j.conbuildmat.2013.08.068>

HARPER, Zachary S.; CONSOLAZIO, Gary R. Calculation Method for Quantifying 
Axial and Roll Stiffnesses of Rectangular Steel-Reinforced Elastomeric Bridge Bearing Pads. Journal of the Transportation Research Board, [s. 1.], n. 2331, p. 3-13, 2013.

HORII, H.; NEMAT-NASSER, S. Brittle failure in compression: Splitting, faulting and brittle-ductile transition. Phil. Trans. Roy. Soc. London: Ser. A. Math. Phys. Sci., [s. 1.], v. 319, n. 1549 , p. 337-374, 1986.

HSU, Thomas T. C. et al. Microcracking of Plain Concrete and the Shape of the Stress-Strain Curve. ACI Journal Proceedings, [s. 1.], v. 60, n. 2, p. 209-224, 1963.

HURFF, J. B. Stability of Precast Prestressed Concrete Bridge Girders Considering Imperfections and Thermal Effects. 2010. Georgia Institute of Technology, [s. 1.], 2010.

HURFF, Jonathan B.; KAHN, Lawrence F. Lateral-Torsional Buckling of Structural Concrete Beams : Experimental and Analytical Study. JOURNAL OF STRUCTURAL ENGINEERING, [s. 1.], v. 138, n. September, p. 1138-1148, 2012.a.

HURFF, Jonathan B.; KAHN, Lawrence F. Rollover stability of precast, prestressed concrete bridge girders with flexible bearings. PCI journal, [s. 1.], p. 96-107, 2012. b.

ILLICH, Günther; TUE, Nguyen Viet; FREYTAG, Bernhard. Schlanke vorgespannte Stützen aus UHPC - Experimentelle Untersuchung und Nachrechnung. Beton- und Stahlbetonbau, [s. 1.], v. 109, p. 534-543, 2014.

JCSS, JCSS. Probabilistic model code. Joint Committee on Structural Safety, [s. 1.], 2001.

KHAN, M. I.; ABBAS, Y. M.; FARES, G. Review of high and ultrahigh performance cementitious composites incorporating various combinations of fibers and ultrafines. Journal of King Saud University - Engineering Sciences, [s. 1.], v. 29, n. 4, p. 339-347, 2017. Disponível em: <http://dx.doi.org/10.1016/j.jksues.2017.03.006>

KIM, Jung Jin et al. Influence of sand to coarse aggregate ratio on the interfacial bond strength of steel fibers in concrete for nuclear power plant. Nuclear Engineering and Design, [s. 1.], v. 252, p. 1-10, 2012. Disponível em: <http://dx.doi.org/10.1016/j.nucengdes.2012.07.004> 
KRAHL, P. A. Instabilidade lateral de vigas pré-moldadas em situações transitórias. 2014. Escola de Engenharia de São Carlos - USP, [s. 1.], 2014.

KRAHL, P. A.; LIMA, M. C. V.; EL DEBS, K. M. Recommendations for verifying lateral stability of precast beams in transitory phases. IBRACON Structural and Materials Journal, [s. 1.], p. 1-20, 2015.

KRAHL, Pablo Augusto; CARRAZEDO, Ricardo; EL DEBS, Khalil Mounir. Rollover stability of precast concrete beams supported by elastomeric bearing pads. Ibracon structures and materials journal, [s. 1.], v. 10, n. 6, p. 1192 - 1204, 2017.

KRAHL, Pablo Augusto; CARRAZEDO, Ricardo; EL DEBS, Mounir Khalil. Lateral buckling analysis of precast UHPFRC beams. In: 4TH INTERNATIONAL SYMPOSIUM ON ULTRA-HIGH PERFORMANCE CONCRETE AND HIGH PERFORMANCE CONSTRUCTION MATERIALS 2016, Anais... [s.l: s.n.]

KRAHL, Pablo Augusto; CARRAZEDO, Ricardo; EL DEBS, Mounir Khalil. Mechanical damage evolution in UHPFRC: experimental and numerical investigation. Engineering Structures, [s. 1.], v. 170, p. 63-77, 2018.

KRAVCHUK, Roman; LANDIS, Eric N. Acoustic emission-based classification of energy dissipation mechanisms during fracture of fiber-reinforced ultra-high-performance concrete. Construction and Building Materials, [s. 1.], v. 176, p. 531-538, 2018. Disponível em: <https://doi.org/10.1016/j.conbuildmat.2018.05.039>

KUPFER, H.; HILSDORF, H. K.; RUSCH, H. Behavior of concrete under biaxial stresses, 1969.

LASZLO, George; IMPER, Richard R. Handling and shipping of long span bridge beams. PCI Journal, [s. 1.], v. 32, n. 6, p. 86-101, 1987.

LEBELLE, P. Stabilité élastique des poutres en béton précontraint a l'égard de déversement latéral. Ann. Batiment et des Travaux Publics, [s. 1.], v. 141, p. 780-830, 1959.

LECHNER, Thomas; FISCHER, Oliver. Stabilitätsverhalten von schlanken Wandscheiben aus Normal- und Ultrahochleistungsbeton. Beton- und Stahlbetonbau, [s. 
1.], v. 110, n. 5, p. 328-339, 2015. Disponível em: <http://doi.wiley.com/10.1002/best.201500011>

LEE, J. H.; FENVES, G. L. Plastic-damage model for cyclic loading of concrete structures. J. Eng. Mech. (ASCE), [s. 1.], v. 124, n. 8, p. 892-900, 1998.

LEE, Ji-Hyung et al. Biaxial tension-compression strength behaviour of UHPFRC in-plane elements. Materials and Structures, [s. 1.], v. 50, n. 1, p. 20, 2017. a. Disponível em: <http://link.springer.com/10.1617/s11527-016-0918-1>

LEE, Jong-han. Evaluation of the lateral stability of precast beams on an elastic bearing support with a consideration of the initial sweep. Engineering Structures, [s. 1.], v. 143, p. 101-112, 2017. Disponível em: <http://dx.doi.org/10.1016/j.engstruct.2017.04.006>

LEE, Jong-han et al. Rollover instability of precast girders subjected to wind load. Magazine of Concrete Research, [s. 1.], v. 69, n. 2, p. 68-83, 2017. b.

LEE, Yun; KANG, Su Tae; KIM, Jin Keun. Pullout behavior of inclined steel fiber in an ultra-high strength cementitious matrix. Construction and Building Materials, [s. 1.], v. 24, n. 10, p. 2030-2041, 2010. Disponível em: <http://dx.doi.org/10.1016/j.conbuildmat.2010.03.009>

LEMAITRE, Jean; CHABOCHE, Jean-Louis. Mechanics of solid materials. ed. 1 ed. Cambridge, UK: Cambridge University Press, 1990.

LI, Chao; HAO, Hong; BI, Kaiming. Numerical study on the seismic performance of precast segmental concrete columns under cyclic loading. Engineering Structures, [s. 1.], v. 148, p. 373-386, $2017 . \quad$ Disponível em: <http://dx.doi.org/10.1016/j.engstruct.2017.06.062>

LI, Victor C.; WU, Hwai-Chung; CHAN, Yin-Wen. Effect of Plasma Treatment of Polyethylene Fibers on Interface and ementitious Composite Properties. Journal of the American Ceramic Society, [s. 1.], v. 79, n. 3, p. 700-704, 1996.

LIMA, M. C. V. Contribuição ao estudo da instabilidade lateral de vigas prémoldadas. 2002. Escola de Engenharia de São Carlos - USP, [s. 1.], 2002. 
LIMA, M. C. V; EL DEBS, M. K. Numerical and experimental analysis of lateral stability in precast concrete beams. Magazine of Concrete Research, [s. 1.], v. 10, p. 635647, 2005.

LUBLINER, J. et al. A plastic-damage model for concrete. Journal of Solids and Structures, [s. 1.], v. 25, p. 299-326, 1989.

MANDAL, P.; CALLADINE, C. R. Lateral-torsional buckling of beams and the Southwell plot. International Journal of Mechanical Sciences, [s. 1.], v. 44, n. 12, p. $2557-$ $2571,2002$.

MANSUR, M. A.; CHIN, M. S.; WEE, T. H. Stress-strain relationship of highstrength fiber concrete in compression. Journal of Materials in Civil Engineering, [s. 1.], v. 11, n. February, p. 21-29, 1999.

MARTIN-SANZ, Henar; CHATZI, Eleni; BRÜHWILER, Eugen. The use of Ultra High Performance Fibre Reinforced cement-based composites in rehabilitation projects: a review. Proceedings of the 9th International Conference on Fracture Mechanics of Concrete and Concrete Structures, [s. 1.], 2016. Disponível em: <http://framcos.org/FraMCoS-9/Full-Papers/219.pdf>

MAST, R. F. Lateral stability of long prestressed concrete beams, part 1. PCI Journal, [s. 1.], v. 34, p. 34-53, 1989.

MAST, R. F. Lateral bending test to destruction of a $149 \mathrm{ft}$ prestressed concrete Ibeam. PCI journal, [s. 1.], v. 39, n. 4, p. 54-62, 1994.

MAST, Robert. Lateral stability of long prestressed concrete beams - parte 2. PCI Journal, [s. 1.], v. special re, p. 70-88, 1993.

MAZARS, Jacky. A description of micro- and macroscale damage of concrete structures. Engineering Fracture Mechanics, [s. 1.], v. 25, n. 5-6, p. 729-737, 1986.

MCGORMLEY, Jonathan C.; LINDENBERG, Richard E. Development of Guidelines for Transportation of Long Prestressed Concrete Girders. [s.l: s.n.]. Disponível em: <http://www.ltrc.lsu.edu/downloads.html>. 
MECK, H. R. Experimental evaluation of lateral buckling. Journal of the Engineering Mechanics Division, [s. 1.], v. 103, n. 2, p. 331-337, 1977.

MEHTA, P. Kumar; MONTEIRO, Paulo J. M. Concrete: Microstructure, Properties, and Materials. 4. ed. [s.1.] : McGraw Hill Professional, 2013.

MULLER, Jean. Lateral Stability of Precast Members During Handling and Placing. PCI journal, [s. 1.], v. 7, n. 1, p. 20-31, 1962.

NAAMAN, Antoine E.; WILLE, Kay. The path to ultra-high performance fiber reinforced concrete (UHP-FRC): five decades of progress. In: PROCEEDINGS OF HIPERMAT 2012, Anais... [s.1: s.n.]

NEVILLE, A. M. Properties of Concrete. [s.1.] : Prentice Hall, 2012.

OESCH, Tyler Scott. Investigation of Fiber and Cracking Behavior for Conventional and Ultra-high Performance Concretes Using X-Ray Computed Tomography. 2015. University of Illinois at Urbana-Champaign, [s. 1.], 2015.

OESTERLE, R. G. et al. Investigation of Red Mountain Freeway Bridge Girder Collapse. [s.l: s.n.].

OSORIO, Edison; BAIRÁN, Jesuús M.; MARÍ, Antonio R. Lateral behavior of concrete under uniaxial compressive cyclic loading. Materials and Structures, [s. 1.], v. 46, p. 709-724, 2013.

PASCHALIS, Spyridon A.; LAMPROPOULOS, Andreas P. Ultra-highperformance fiber-reinforced concrete under cyclic loading. ACI Materials Journal, [s. 1.], v. 113, n. 4, p. 419-427, 2016.

PEART, Walter L.; RHOMBERG, Edward J.; JAMES, Ray W. Buckling of suspended cambered girders. Journal of Structural Engineering, [s. 1.], v. 118, n. 2, p. 505-528, 1992.

PLAUT, Raymond H.; MOEN, Cristopher D. Analysis of Elastic, Doubly Symmetric, Horizontally Curved Beams during Lifting. Journal of Structural Engineering, [s. 1.], v. 139, n. 1, p. 39-46, 2012. Disponível em: 
<http://ascelibrary.org/doi/10.1061/\%28ASCE\%29ST.1943-541X.0000612>

PLAUT, Raymond H.; MOEN, Cristopher D. Stability of unbraced concrete beams on bearing pads including wind loading. Engineering Structures, [s. 1.], v. 69, p. 246-254, 2014. Disponível em: <http://dx.doi.org/10.1016/j.engstruct.2014.03.024>

PREM, Prabhat Ranjan. Influence of curing regime and steel fibres on the mechanical properties of UHPC. Magazine of Concrete Research, [s. 1.], v. 67, n. 18, p. 988-1002, 2015.

REN, G. M. et al. Triaxial compressive behavior of UHPCC and applications in the projectile impact analyses. Construction and Building Materials, [s. 1.], v. 113, p. 1-14, 2016.

REN, G. M. et al. Effects of steel fiber content and type on static mechanical properties of UHPCC. Construction and Building Materials, [s. 1.], v. 163, p. 826-839, 2018.

REVATHI, P.; MENON, Devdas. Estimation of Critical Buckling Moments in Slender Reinforced Concrete Beams. ACI STRUCTURAL JOURNAL, [s. 1.], v. 103, p. 296-303, 2006.

RUSSELL, Henry G.; GRAYBEAL, Benjamin A. Ultra-High Performance Concrete : A State-of-the-Art Report for the Bridge Community. [s. 1.], n. June, p. 171, 2013.

SANCHEZ, Florence; INCE, Chantal. Microstructure and macroscopic properties of hybrid carbon nanofiber/silica fume cement composites. Composites Science and Technology, [s. 1.], v. 69, n. 7-8, p. 1310-1318, 2009. Disponível em: <http://dx.doi.org/10.1016/j.compscitech.2009.03.006>

SANT, J. K.; BLETZACKER, R. W. Experimental Study of Lateral Stability of Reinforced Concrete Beams. ACI Structural Journal, [s. 1.], v. 58, n. 33, p. 713-736, 1961.

SCHMIDT, Angela; CURBACH, Manfred. Zentrische Druckversuche an schlanken UHPC-Stützen. Beton- und Stahlbetonbau, [s. 1.], v. 111, n. 9, p. 588-602, 2016. Disponível em: 〈http://doi.wiley.com/10.1002/best.201600027> 
SCHMIDT, Angela; CURBACH, Manfred. Design optimization to increase the (buckling) stability of concrete columns. Structural Concrete, [s. 1.], v. 18, n. 5, p. 680692, 2017.

SERNA, Pedro et al. Pasarela de hormigón de muy alto rendimiento sobre el Barranco de las Ovejas (Alicante). Hormigón y Acero, [s. 1.], v. 66, n. 275, p. 23-42, 2015.

SFER, Domingo et al. Study of the Behavior of Concrete under Triaxial Compression. Journal of Engineering Mechanics, [s. 1.], v. 128, n. 2, p. 156-163, 2002.

SHAH, Surendra P.; SWARTZ, Stuart E.; OUYANG, Chengsheng. Fracture Mechanics of Concrete: Applications of Fracture Mechanics to Concrete, Rock and Other Quasi-Brittle Materials. [s.1.] : John Wiley \& Sons, 1995.

SHI, Caijun et al. A review on ultra high performance concrete: Part I. Raw materials and mixture design. Construction and Building Materials, [s. 1.], v. 101, p. 741-751, 2015. Disponível em: <http://dx.doi.org/10.1016/j.conbuildmat.2015.08.095>

SOBUZ, H. R. et al. Manufacturing ultra-high performance concrete utilising conventional materials and production methods. CONSTRUCTION \& BUILDING MATERIALS, [s. 1.], v. 111, p. 251-261, 2016. Disponível em: <http://dx.doi.org/10.1016/j.conbuildmat.2016.02.102>

SOHAIL, Muazzam Ghous et al. Advancements in Concrete Mix Designs: HighPerformance and Ultrahigh-Performance Concretes from 1970 to 2016. Journal of Materials in Civil Engineering, [s. 1.], v. 30, n. 3, p. 04017310, 2018. Disponível em: <http://ascelibrary.org/doi/10.1061/\%28ASCE\%29MT.1943-5533.0002144>

SOUTHWELL, R. V. On the analysis of experimental observations in problems of elastic stability. Proceedings of the Royal Society, [s. 1.], v. 135, p. 601-616, 1932.

SOVJÁKA, Radoslav; VOGEL, Filip; BECKMANN, Birgit. Triaxial compressive strength of ultra-high performance concrete. Acta Polytechnica, [s. 1.], v. 53, n. 6, p. 901905, 2013.

SPECK, Kerstin. Beton unter mehraxialer beanspruchung ein materialgesetz für hochleistungsbetone unter kurzzeitbelastung. 2008. University of Dresden, [s. 1.], 2008. 
STRATFORD, T. J.; BURGOYNE, C. J. The toppling of hanging beams. International journal of solids and structures, [s. 1.], v. 37, n. 26, p. 3569-3589, 2000.

SWANN, R. A. Reader's Comment to article on "Lateral Stability of Long Prestressed Concrete Beams,”. PCI Journal, [s. 1.], v. 16, n. 6, p. 85-89, 1971.

SWANN, R. A.; GODDEN, W. G. The lateral buckling of concrete beams lifted by cables. The Structural Engineer, [s. 1.], v. 44, n. 1, p. 21-33, 1966.

TAYEH, Bassam A. et al. Utilization of Ultra-High Performance Fibre Concrete (UHPFC) for Rehabilitation - a Review. Procedia Engineering, [s. 1.], v. 54, p. 525 - 538, 2013.

THOMAS, R. J.; SORENSEN, Andrew D. Review of strain rate effects for UHPC in tension. Construction and Building Materials, [s. 1.], v. 153, p. 846-856, 2017. Disponível em: <http://dx.doi.org/10.1016/j.conbuildmat.2017.07.168>

TIMOSHENKO, S.; GERE, J. Theory of Elastic Stability. $3^{\text {a }}$ edition ed. New York: McGraw-Hill, 1988.

TOUTLEMONDE, François; DELORT, Michel. The newly enforced French Standard for UHPFRC specification, performance, production and conformity. In: PROCEEDINGS OF HIPERMAT 2016 4TH INTERNATIONAL SYMPOSIUM ON ULTRA-HIGH PERFORMANCE CONCRETE AND HIGH PERFORMANCE MATERIALS 2016, Anais... [s.l: s.n.]

TRAHAIR, Nicholas. Torsion equations for lateral buckling. Engineering Structures, [s. 1.], v. 128, p. 161-165, 2016. Disponível em: <http://dx.doi.org/10.1016/j.engstruct.2016.09.039>

TRAHAIR, Nick. Flexural-Torsional Buckling of Structures. [s.1.] : Taylor \& Francis, 1993.

TRAINOR, Kevin J. et al. Measurement of Energy Dissipation Mechanisms in Fracture of Fiber-Reinforced Ultrahigh-Strength Cement-Based Composites. [s. 1.], v. 139, n. July, p. 771-779, 2013. a. 
TRAINOR, Kevin J. et al. Measurement of Energy Dissipation Mechanisms in Fracture of Fiber-Reinforced Ultrahigh-Strength Cement-Based Composites. Journal of Engineering Mechanics, [s. 1.], v. 139, n. July, p. 771-779, 2013. b.

VAN MIER, J. G. M. Fracture processes of concrete. [s.l.] : CRC Press, 1997.

VAN MIER, Jan. Concrete Fracture: A Multiscale Approach. [s.1.] : Taylor \& Francis, 2012. Disponível em: <http://www.crcnetbase.com/doi/book/10.1201/b12968>

WANG, Dehui et al. A review on ultra high performance concrete: Part II. Hydration, microstructure and properties. Construction and Building Materials, [s. 1.], v. 96, p. 368377, 2015. Disponível em: <http://dx.doi.org/10.1016/j.conbuildmat.2015.10.088>

WEE, T. H.; CHIN, M. S.; MANSUR, M. A. Stress-Strain Relationship of HighStrength Concrete in Compression. Journal of materials in civil engineering, [s. 1.], v. 8, n. 2, p. 70-76, 1996.

WILLE, K.; EL-TAWIL, S.; NAAMAN, A. E. Properties of strain hardening ultra high performance fiber reinforced concrete (UHP-FRC) under direct tensile loading. Cement and Concrete Composites, [s. 1.], v. 48, p. 53-66, 2014. Disponível em: <http://www.sciencedirect.com/science/article/pii/S0958946514000055>

WILLE, Kay; KIM, Dong Joo; NAAMAN, Antoine E. Strain-hardening UHP-FRC with low fiber contents. Materials and Structures/Materiaux et Constructions, [s. 1.], v. 44, n. 3, p. 583-598, 2011.

WU, Zemei et al. Uniaxial Compression Behavior of Ultra-High Performance Concrete with Hybrid Steel Fiber. Journal of Materials in Civil Engineering, [s. 1.], v. 28, n. 12, p. 1-7, 2016.

WU, Zemei; KHAYAT, Kamal Henri; SHI, Caijun. Effect of nano-SiO 2 particles and curing time on development of fiber-matrix bond properties and microstructure of ultrahigh strength concrete. Cement and Concrete Research, [s. 1.], v. 95, p. 247-256, 2017. Disponível em: <http://linkinghub.elsevier.com/retrieve/pii/S0008884616311164>

WU, Zemei; SHI, Caijun; KHAYAT, K. H. Influence of silica fume content on microstructure development and bond to steel fiber in ultra-high strength cement-based 
materials (UHSC). Cement and Concrete Composites, [s. 1.], v. 71, p. 97-109, 2016. Disponível em: <http://dx.doi.org/10.1016/j.cemconcomp.2016.05.005>

YOO, Doo-Yeol; PARK, Jung-Jun; KIM, Sung-Wook. Fiber pullout behavior of HPFRCC: Effects of matrix strength and fiber type. Composite Structures, [s. 1.], v. 174, p. 2017. 2636, Disponível em: <http://linkinghub.elsevier.com/retrieve/pii/S0263822317306463>

YOO, Doo-Yeol; YOON, Young-Soo. A Review on Structural Behavior, Design, and Application of Ultra-High-Performance Fiber-Reinforced Concrete. International Journal of Concrete Structures and Materials, [s. 1.], v. 10, n. 2, p. 125-142, 2016. Disponível em: 〈http://link.springer.com/10.1007/s40069-016-0143-x>

YOO, Doo Yeol; BANTHIA, Nemkumar. Mechanical properties of ultra-highperformance fiber-reinforced concrete: A review. Cement and Concrete Composites, [s. 1.], v. 73, p. 267-280, 2016. Disponível em: <http://dx.doi.org/10.1016/j.cemconcomp.2016.08.001>

YOO, Doo Yeol; BANTHIA, Nemkumar. Mechanical and structural behaviors of ultra-high-performance fiber-reinforced concrete subjected to impact and blast. Construction and Building Materials, [s. 1.], v. 149, p. 416-431, 2017. Disponível em: <http://dx.doi.org/10.1016/j.conbuildmat.2017.05.136>

YU, R.; SPIESZ, P.; BROUWERS, H. J. H. Cement and Concrete Research Mix design and properties assessment of Ultra-High Performance Fibre Reinforced Concrete ( UHPFRC ). [s. 1.], v. 56, p. 29-39, 2014.

ZHANG, J.; LI, J. Investigation into Lubliner yield criterion of concrete for 3D simulation. Engineering Structures, [s. 1.], v. 44, p. 122-127, 2012.

ZHOU, Jiajia et al. Mechanical Behavior of Fiber-Reinforced Engineered Cementitious Composites in Uniaxial Compression. J. Mater. Civ. Eng., [s. 1.], v. 27, n. 1, p. 1-10, 2015.

ZOHREVAND, Pedram; MIRMIRAN, Amir. Behavior of ultrahigh-performance concrete confined by fiber-reinforced polymers. Journal of Materials in Civil 
Engineering, [s. 1.], v. 23, n. 12, p. 1727-1734, 2011. 